\title{
Determination of Bioimpedance Using Multi-Frequency \\ Bio-electrical Impedance Analysis \\ by
}

Satyanarayana Shashank Brahmandlapalli

A thesis submitted to the Faculty of Graduate and

Postdoctoral Affairs in partial fulfillment of the

requirements for the degree of

Masters Of Applied Science

in

Electrical and Computer Engineering

Carleton University

Ottawa, Ontario

(C)2016

Satyanarayana Shashank Brahmandlapalli 


\section{Abstract}

A current source circuit and a voltage acquisition circuit along with a postprocessing rectifier circuit are designed to determine bioimpedance of a biological subject.

An operational amplifier based current source with a simulated output impedance of $6.7 \mathrm{M} \Omega$ and operational at frequencies under $100 \mathrm{kHz}$ is designed to inject current into the subject. An instrumentation amplifier operating at unity gain with a simulated CMRR of $-111.4 \mathrm{~dB}$ and DC power consumption of $477.7 \mu \mathrm{W}$ is used to acquire the voltage response of the subject. A diode-less rectifier circuit that eliminates the loss in voltage due to diode threshold voltage during rectification is designed.

Layouts for the sub-circuits have been designed and developed using the Cadence layout tool, Vitruoso, and integrated to develop the IC layout. When tested, the operational amplifier is found to oscillate due to inadequate phase margin. 


\section{Acknowledgements}

Researching a topic, designing an integrated circuit and the IC layout, taping it out, testing the chips upon their fabrication, writing the thesis and defending the thesis has been a fierce and profound journey. Having very little background on IC design and very limited experience in circuits, this thesis couldn't have been possible without the support of a few people in my life. With all humility, I'd like take this opportunity to acknowledge the people and appreciate their support.

First of all, I'd like to thank my parents, Mr. B Rama Rao and Ms. B N S Prasanna Laxmi. It can be categorically stated that without their financial and emotional support, this thesis would've remained an un-true dream.

Second of all, I'd like to thank my supervisor, Dr. Leonard MacEachern, for his support and confidence in me through out the course of the thesis. His professional expertise and personality taught me more than just designing circuits. It is an honor to have worked with him under his supervision and would like to continue to learn from him in the future too.

Thirdly, I'd like thank my friend, Mr. Niranjan B Ramesh, for sharing his knowledge of integrated circuits and encouraging me throughout the thesis.

Fourthly, I'd like to thank Dr. Tadeus Kwasniewski for his extended office hours and after-class discussions which taught me a lot. This paved way for my profes- 
sional development.

I'd like to thank Mr. Nagui Mikhail for technical and lab support. His studentfriendly attitude and expertise in subject makes him approachable and reliable. A special thanks to Blazenka Power for her help with proof-reading and other administrative work. Also, I'd like to thank Anna Lee for the administrative support that has been extended to me from her side.

Last but not the least, I'd like to acknowledge the effort of all those people who have directly or indirectly, knowingly or un-knowingly contributed towards the thesis. 


\title{
List Of Acronyms
}

\author{
ASIC : Application Specific Integrated Circuit \\ ESD : Electrostatic Discharge \\ SUT : Subject Under Test \\ IC : Integrated Circuit \\ PCB : Printed Circuit Board \\ MOSFET : Metal Oxide Semiconductor Field Effect Transistor \\ DC : Direct Current \\ AC : Alternating Current \\ BIA: Bioelectrical Impedance Analysis \\ CMRR - Common Mode Rejection Ratio \\ CMR - Common Mode Rejection \\ DRC - Design Rule Check \\ LVS - Layout Versus Schematic \\ DFM - Design For Manufacturability \\ WHO - World Health Organization \\ RMS - Root Mean Square
}




\section{Contents}

1 Introduction 1

1.1 Thesis Outline . . . . . . . . . . . . . . . . 4

2 Theory of Bioimpedance $\quad 6$

2.1 Bioimpedance Measurement . . . . . . . . . . . . . . . . 6

2.1.1 Electrical System for Bioimpedance Measurement . . . . . . 6

2.1.2 Practical Considerations . . . . . . . . . . . . . . . 12

2.2 Proposed Bioimpedance System Architecture . . . . . . . . . . . . . 13

2.2.1 Architecture Overview . . . . . . . . . . . . . . 14

2.3 Proposed Bioimpedance System Specifications . . . . . . . . . . . . 16

2.3 .1 Current Injection . . . . . . . . . . . . . . . . . . 16

2.3.2 Voltage Measurement . . . . . . . . . . . . . 18

2.4 Hardware Modules of the Bioimpedance System . . . . . . . . . . . 22

2.4 .1 Current Source . . . . . . . . . . . . . . . . 22

2.4.2 Voltage Measuring Circuit . . . . . . . . . . . . . . 38

2.4.3 Post Signal Processing Circuit . . . . . . . . . . . . . . 44

2.5 Literature Review of Bioimpedance Circuits . . . . . . . . . . . 48

2.5.1 AFE4300 . . . . . . . . . . . . . . 49 
2.5.2 HM301D . . . . . . . . . . . . . . . 51

2.6 Chapter Summary . . . . . . . . . . . . . . . 52

3 Design and Simulations $\quad 56$

3.1 Operational Amplifier . . . . . . . . . . . . . . . . 56

3.1 .1 Circuit Description . . . . . . . . . . . 57

3.1.2 Circuit Operation . . . . . . . . . . . . . . 61

3.1 .3 Differential Gain . . . . . . . . . . . . . . . . . 74

3.1.4 Common Mode Rejection . . . . . . . . . . . . . 78

3.1.5 Common Mode Rejection Ratio . . . . . . . . . . . . . . . . 80

3.1 .6 Output Impedance . . . . . . . . . . . . . . . . . 81

3.1.7 Input and Output Common Mode Range . . . . . . . . . . . 82

3.1 .8 Slew Rate . . . . . . . . . . . . . . . . . . . . . 88

3.1 .9 Non-linearity . . . . . . . . . . . . . . . . . . 90

3.1 .10 Offset Voltage . . . . . . . . . . . . . . . . . 95

3.1 .11 Power Consumption . . . . . . . . . . . . . 96

3.2 Current Source . . . . . . . . . . . . . . . . . . . . . . 97

3.2.1 Basic Howland Current Source . . . . . . . . . . . . . . . . . 98

3.2.2 Modified Howland Current Source . . . . . . . . . . . . . . 107

3.2.3 Mirrored Modified Howland Current Source . . . . . . . . . 117

3.3 Instrumentation Amplifier . . . . . . . . . . . . . . . . . . . 132

3.3.1 Operating Gain . . . . . . . . . . . . . . . . 137

3.3.2 Common Mode Rejection Ratio . . . . . . . . . . . . . . . . 140

3.3.3 Non-linearity . . . . . . . . . . . . . . . . 143

3.3.4 Offset voltages . . . . . . . . . . . . . . . . . 145 
3.3.5 Power consumption . . . . . . . . . . . . . . . . . . 147

3.4 Post Processing Circuit . . . . . . . . . . . . . . . . . . 147

3.4.1 Circuit Description and Operation . . . . . . . . . . . . 148

3.4.2 Discussion . . . . . . . . . . . . . . . . . . . 152

3.5 Bioimpedance System . . . . . . . . . . . . . . . . . . 157

3.5.1 Bioimpedance System Schematic . . . . . . . . . . . 157

3.5.2 Simulation and Discussion . . . . . . . . . . . . 159

3.6 Layouts . . . . . . . . . . . . . . . . . . . . . . . . 161

3.7 Chapter Summary . . . . . . . . . . . . . . . . 167

4 Testing and Debugging $\quad 170$

4.1 Testing . . . . . . . . . . . . . . . . . . . . . . . 171

4.1 .1 Chip Setup . . . . . . . . . . . . . . 171

4.1 .2 ESD Caution . . . . . . . . . . . . . . . 172

4.1 .3 Testing Equipment . . . . . . . . . . . . . . . 173

4.1.4 Testing Methodology . . . . . . . . . . . . . . 174

4.1.5 Test Results and Debugging . . . . . . . . . . . . . 174

4.2 Failure Analysis . . . . . . . . . . . . . . . . . . . . . . . 181

4.2.1 Design Issue I - Phase Margin . . . . . . . . . . . . . . . . 181

4.2.2 Design Issue II - Output Stage . . . . . . . . . . . . 183

5 Operational Amplifier Re-designed $\quad 186$

5.1 Circuit Description . . . . . . . . . . . . . . . 186

5.2 Simulations . . . . . . . . . . . . . . . . . . . 190

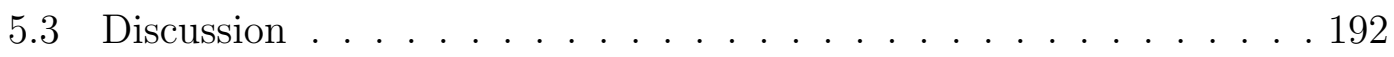


5.4 Chapter Summary . . . . . . . . . . . . . . . . . 193

Appendix A Monte Carlo Simulations

A.1 Differential Gain . . . . . . . . . . . . . . . . . 199

A.2 Common Mode Rejection . . . . . . . . . . . . . . . . . 206 


\section{List of Figures}

2.1 Bi-polar bioimpedance measurement technique . . . . . . . . . . 7

2.2 Electrical model of bi-polar method . . . . . . . . . . . . . . . . 8

2.3 Tetra-polar method . . . . . . . . . . . . . . . . . . . 10

2.4 Electrical model of tetra-polar method . . . . . . . . . . . . . . 11

2.5 Bioimpedance measurement system . . . . . . . . . . . . . . . 15

2.6 Basic current mirror f . . . . . . . . . . . . . . . . . . . . 24

2.7 Cascode current mirror current source . . . . . . . . . . . . . . . 26

2.8 Current conveyor current source . . . . . . . . . . . . . . . 27

2.9 Voltage controlled current source . . . . . . . . . . . . . . 28

2.10 Basic Howland current source . . . . . . . . . . . . . . . . . . 30

2.11 Modified Howland Current Source . . . . . . . . . . . . . . . 34

2.12 Mirrored modified Howland current source . . . . . . . . . . . . . . 37

2.13 Difference amplifier . . . . . . . . . . . . . . . . . . . . 39

2.14 Instrumentation amplifier f . . . . . . . . . . . . . . . . . 42

2.15 Gate Rectifier . . . . . . . . . . . . . . . . . . 46

2.16 Gate Rectifier HWR . . . . . . . . . . . . . . . . . 47

2.17 Gated Rectifier HWR . . . . . . . . . . . . . . . . . . . . 48 
3.14 -stage Operational Amplifier . . . . . . . . . . . . . . . . . 59

3.2 A typical PMOS differential gain stage of an op-amp with NMOS active loads . . . . . . . . . . . . . . . . . . 61

3.3 The small signal model of a typical differential gain stage of an

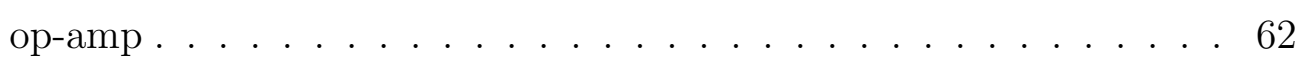

3.4 A typical differential gain stage of an op-amp with current mirror

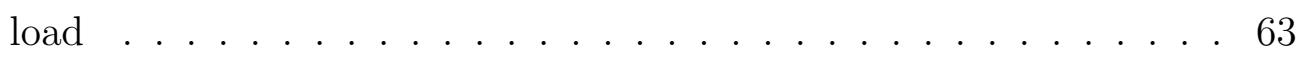

3.5 The small signal model of a typical differential gain stage of an op-amp with current mirror load . . . . . . . . . . . . . . 64

3.6 Diff pair active load . . . . . . . . . . . . . . . 65

3.7 Diff pair with current mirror load . . . . . . . . . . . . 66

3.8 Transient behavior of the differential gain stage with active load circuit ............................. 67

3.9 Transient behavior of the differential gain stage with current mirror load circuit f. . . . . . . . . . . . . . . 6 68

3.10 Cascaded differential gain stages . . . . . . . . . . . . . 70

3.11 Transient analysis of 2 cascaded differential gain stages with high impedance ouptut from 1st stage connected to the diode-connected low impedance branch of the 2 nd stage . . . . . . . . . . . 71

3.12 Cascaded gain stages with low impedance input connected to low impedance output branch . . . . . . . . . . . . . . 72

3.13 Transient analysis of 2 cascaded differential gain stages with low impedance ouptut from 1st stage connected to the diode-connected low impedance branch of the 2 nd stage . . . . . . . . . . . 73 
3.14 Differential gain of the operational amplifier . . . . . . . . . . 76

3.15 Common mode gain of the operational amplifier . . . . . . . . . . 79

3.16 Differential stage of the designed operational amplifier . . . . . . 83

3.17 Input common mode range of the operational amplifier . . . . . . . 85

3.18 Output voltage plot versus common mode input voltage $\ldots$. . . . 87

3.19 Slew rate of the operational amplifier . . . . . . . . . . . . 89

3.20 Output,Y, vs input,X, of a linear system . . . . . . . . . . . 91

3.21 Conceptual illustration of non-linearity . . . . . . . . . . . . . . 92

3.22 Non-linearity assessment using straight line method . . . . . . . . . 94

3.23 Voltage Offset f . . . . . . . . . . . . . . 96

3.24 Basic Howland Current Source . . . . . . . . . . . . . . . . . . 98

3.25 Transient behavior of the BHCS - current plots . . . . . . . . 100

3.26 Transient behavior of BHCS - voltage plots . . . . . . . . . . . 101

3.27 Current vs. Frequency plot of BHCS . . . . . . . . . . . . 102

3.28 Output impedance plots for BHCS with matched resistors . . . . 103

3.29 Output impedance plots with mismatched resistors . . . . . . . . 104

3.30 Output current with reduced input and feedback resistances . . . . 106

3.31 Modified Howlandc Current Source . . . . . . . . . . . . . . . . . 108

3.32 Output currents - MHCS . . . . . . . . . . . . . . 109

3.33 Output currents for varied differential input - MHCS . . . . . . . 110

3.34 Increased output current with reduced gain setting resistor value -

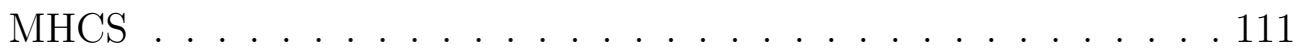

3.35 Limited compliance of the modified Howland current source . . . . 113

3.36 Limited compliance of MHCS - differential input is $600 \mathrm{mV}$. . . . . 114 
3.37 Limited compliance of MHCS - differential input is $700 \mathrm{mV}$. . . . . 116

3.38 Mirrored modified Howland current source schematic . . . . . . . . 119

3.39 Load current transient plot of the MMHCS . . . . . . . . . 121

3.40 Load current plots for varying differential input voltages . . . . . . 122

3.41 Load current plots for varying gain setting resistor resistance . . 123

3.42 Output impedance of MMHCS . . . . . . . . . . . . . . 124

3.43 Output currents of varying loads . . . . . . . . . . . . . 125

3.44 Extended compliance of the mirrored modified Howland current

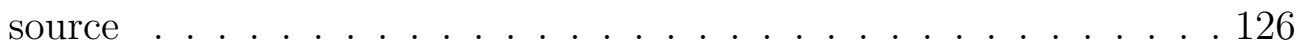

3.45 Discrete Fourier Transform (DFT) spectrum of output current of the MMHCS . . . . . . . . . . . . . . . . . . . . . . 128

3.46 Output current in the harmonics - Fourier spectrum . . . . . . . 129

3.47 Output current vs, frequency plot of MMHCS . . . . . . . . . 131

3.48 Instrumentation amplifier schematic . . . . . . . . . . . 133

3.49 Instrumentation amplifier - Transient simulation . . . . . . . 136

3.50 Instrumentation amplifier - Transient simulation for gain, G, equal

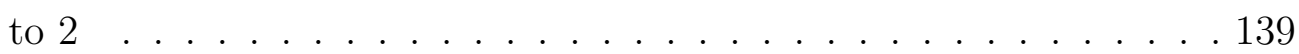

3.51 Common mode rejection - Transient simulation . . . . . . . . . . 141

3.52 Output plot with sweeping input - instrumentation amplifier . . . . 144

3.53 Output slope plot of the instrumenation amplifier . . . . . . . . 145

3.54 Output frequency spectrum of the instrumenation amplifier . . . . 146

3.55 Gate Rectifier Schematic . . . . . . . . . . . . . . . . . 149

3.56 Half-wave rectifier transient behavior . . . . . . . . . . . . . . 150

3.57 Full-wave rectifier transient behavior . . . . . . . . . . . . . . 151 
3.58 Distorted and Un-distorted HWR outputs of a diode-based and a diode-less rectifier respectively . . . . . . . . . . . . 153

3.59 DC Analysis of a transmission gate . . . . . . . . . . . . 154

3.60 Bandwidth of the transmission gate . . . . . . . . . . . . 155

3.61 Bioimpedance system schematic . . . . . . . . . . . . . 158

3.62 Bioimpedance system transient behavior . . . . . . . . . . . . 160

3.63 Operational amplifier layout . . . . . . . . . . . . . . . 164

3.64 Instrumentation amplifier layout f . . . . . . . . . . . 165

3.65 ICGCUAUM layout . . . . . . . . . . . . . . . . 166

4.1 Photograph of the chip . . . . . . . . . . . . . . . . 171

4.2 ICGCUAUM pin diagram . . . . . . . . . . . . . . . 172

4.3 Unity gain buffer with input and expected output signal . . . . . 176

4.4 Unity gain operational amplifier . . . . . . . . . . . . . . . 177

4.5 Oscillating buffer output . . . . . . . . . . . . . . . 178

4.6 Oscillating signal observed . . . . . . . . . . . . . . . . . . . 179

4.7 Gain and Phase Plots . . . . . . . . . . . . . . . . . . . . 182

4.8 Buffer stage - operational amplifier . . . . . . . . . . . . . . . 184

5.1 Operational amplifier schematic - version $2 \ldots . . \ldots 187$

5.2 AC Gain and phase plots of new operational amplifier . . . . . 190

5.3 Differential gain of new operational amplifier . . . . . . . . . . 191

5.4 Common mode rejection of the new operational amplifier . . . . 192

A.1 Monte-carlo Simulation - Differential Gain at $1 \mathrm{kHz}$. . . . . . . 200

A.2 Monte-carlo Simulation - Differential Gain at 5kHz . . . . . . . . 201 
A.3 Monte-carlo Simulation - Differential Gain at 10kHz . . . . . . . . 202

A.4 Monte-carlo Simulation - Differential Gain at 50kHz . . . . . . . 203

A.5 Monte-carlo Simulation - Differential Gain at $75 \mathrm{kHz}$. . . . . . . . 204

A.6 Monte-carlo Simulation - Differential Gain at 100kHz . . . . . . . . 205

A.7 Monte-carlo Simulation - Common mode rejection at $1 \mathrm{kHz}$. . . . . 207

A.8 Monte-carlo Simulation - Common mode rejection at $5 \mathrm{kHz}$. . . . . 208

A.9 Monte-carlo Simulation - Common mode rejection at $10 \mathrm{kHz}$. . . . 209

A.10 Monte-carlo Simulation - Common mode rejection at $50 \mathrm{kHz}$. . . . 210

A.11 Monte-carlo Simulation - Common mode rejection at $75 \mathrm{kHz}$. . . . 211

A.12 Monte-carlo Simulation - Common mode rejection at $100 \mathrm{kHz}$. . . 212

B.1 Transient behavior of the bioimepdance system $-5 \mathrm{kHz} \ldots 216$

B.2 Transient behavior of the bioimepdance system $-50 \mathrm{kHz} \quad \ldots . . .217$

B.3 Transient behavior of the bioimepdance system $-100 \mathrm{kHz}$. . . . . . 218 


\section{List of Tables}

2.1 Op-amp design specifications . . . . . . . . . . . . . 20

2.2 Current source design specifications . . . . . . . . . . . . . . 21

2.3 Voltage measuring circuit design specifications . . . . . . . . . . . 21

2.4 Comparison of Specifications of the IC with AFE4300 and HM301D chips. . . . . . . . . . . . . . . . . . 5 54

2.5 Designed operational amplifier specifications, obtained from simulations, compared with other published operational amplifiers . . . . 55

3.1 Designed Operational amplifier components and their values . . . . 60

3.2 Output current in the harmonics . . . . . . . . . . . . . 129

3.3 Instrumentation amplifier - Resistor values . . . . . . . . . . . . . 134

3.4 Instrumentation amplifier - Gain setting resistor values . . . . . . . 138

3.5 Gain and CMRR values of the instrumentation amplifier . . . . . . 142

3.6 Output voltage in the fundamental and harmonic frequencies . . . . 147

3.7 Transmission gate bandwidth . . . . . . . . . . . . . 156

3.8 Inputs to the BIS system, various parameters and their values used in performing transient analysis. . . . . . . . . . . . . . . 159

3.9 Operational amplifier simulated specifications . . . . . . . . . . 169 
5.1 New Operational amplifier components and their values . . . . . . . 189

5.2 Post-fab new operational amplifier simulated specifications . . . . . 194

A.1 Differential gain, common mode gain and CMRR of the operational amplifier at different frequencies . . . . . . . . . . . . . . . 213

A.2 Corner simulations results for differential gain, common mode gain and CMRR . . . . . . . . . . . . . . . . . . . 214 


\section{Chapter 1}

\section{Introduction}

According to World Health Organization (WHO), worldwide obesity has dramatically increased since 1980. WHO's global estimates state that in 20141.9 billion adults were overweight of which 600 million were obese. Not only is the number of adults suffering obesity or overweight is on the rise, the number of children suffering from either overweight or obesity is increasing significantly. An estimated 41 million children under the age of 5 years were reported to be either overweight or obese. It is observed that obesity and overweight cause more deaths worldwide than underweight.

It is a proven fact that the imbalance in calories consumed and calories spent results in overweight or obesity. Simply stated, an individual with a high intake of energy-dense foods containing high fat composition combined with a sedentary lifestyle, will result in an increase in his body mass index (BMI). The risk factor for cardiovascular diseases, diabetes, musculo-skeletal disorders and some cancers rises with an increase in the BMI which is the direct result of the excess fat present in the individual. In a rapidly changing world and increasing urbanization, mon- 
itoring the food intake and personal health, especially the fat composition, is a necessity. This brings us to the technique of bioimpedance analysis.

Bio-electrical Impedance Analysis (BIA) is generally a simple and non-invasive technique that is widely used in estimating body composition. Contrary to the general perception of bio-electrical impedance analysis directly yielding fat percentage in the human body, bioimpedance analysis actually determines the electrical impedance of a biological subject, termed bioimpedance. The resulting electrical impedance provides a reliable estimate of Total Body Water (TBW). The measured TBW is used in deriving fat mass and fat-free mass in the body tissues.

Bioimpedance [1] is a measure of obstruction to the flow of an alternating current injected into the body tissues due to the tissues constituents. The obstruction offered is both capacitive and resistive in nature, hence the term bioimpedance. The response of the tissues, in terms of conductivity and permittivity, when subjected to electrical excitation, may help us understand the underlying composition, structure and alignment of the tissues.

A few of the many applications of bio-electrical impedance analysis are its applicability in determining cell sizes and their concentration, its use in blood analyzers to measure hematocrit, the study of response of cellular cultures to external agents, cancerous cells recognition and its ease of use in characterizing tissues. Especially, the applicability of bioimpedance analysis in determining the body composition has led to its widespread acceptance commercially, giving rise to products such as health care monitors and body fat analyzers. 
Deduction of bioimpedance [2] necessitates galvanostatic or potentiostatic excitation of a biological tissue at single or multiple frequencies. The technique of applying electrical signals at multiple frequencies and acquiring the response of the subject is termed Electrical Impedance Spectroscopy (EIS).

This thesis is aimed at providing an ASIC solution using 130nm IBM technology in finding bioimpedance of a biological tissue. The circuit designed employs current sources to inject an alternating current of a constant RMS value into a Subject-Under-Test (SUT) and a voltage acquisition circuit to measure the voltage across the subject. Post voltage acquisition signal processing involves on-chip rectification of the measured voltage signal and filtering it off-chip. As a result, a diode-less rectification circuit is proposed and designed.

The rising demand for health-care products and the applicability of bioimpedance in their designs have been major motivating factors for this thesis. 


\subsection{Thesis Outline}

This thesis is structured as follows:

Chapter 1 serves as an introduction to bioimpedance and states the intention of the thesis.

Chapter 2 provides a background on methods of determination of bioimpedance and its practical considerations, followed by the background is the proposed bioimpedance system architecture and rationale behind the specifications of the system and its sub-circuits. The Chapter also discusses the sub-circuits of the system. While current controlled current sources are explained briefly, an in-depth understanding of Howland current sources is presented to better understand the designed circuit and the simulations in Chapter 3. The pros and cons of a difference amplifier, along with an explanation of the instrumentation amplifier, is described. The Chapter concludes with a literature review of the bioimpedance integrated circuits that are commercially available.

Chapter 3 describes the implementation of the system, shows simulations of subcircuits and discusses the results. The Chapter is divided into sections that describe and discuss the designed operational amplifier, current source, voltage measuring circuit and the integrated bioimpedance system. Sub-circuit layouts and the complete IC layout are shown and techniques employed in layout implementation are referenced.

Chapter 4 provides information regarding the different tests performed to validate both the functionality and various modules of the chip as well as a discussion of the results.

Following the results of the chip testing, the operational amplifier is re-designed 
and discussed in the Chapter 5. The main issues of the design are addressed and confirmed with simulations.

Chapter 6 concludes the thesis. 


\section{Chapter 2}

\section{Theory of Bioimpedance}

\subsection{Bioimpedance Measurement}

\subsubsection{Electrical System for Bioimpedance Measurement}

Bioimpedance is the impedance that an injected current experiences when the current is injected into a biological subject. To find an unknown impedance, in this case, bioimpedance, a known alternating current is injected into a biological subject and the drop in voltage across the subject observed is measured. Mathematically speaking, impedance is the ratio of the measured voltage to injected current in the SUT.

$$
\text { Bioimpedance }=\frac{\Delta V_{\text {across } S U T}}{I_{\text {injected }}}
$$

Understandably, the system requires current source circuits, voltage measurement circuits and a post-processing circuit. Two widely used techniques to measure bioimpedance by injecting current into a biological subject and measuring voltage across the subject under test are bi-polar method and tetra-polar method. 


\section{Bi-polar Method}

Bi-polar method (also known as two electode technique) of current injection and voltage measuring technique uses the same pair of electrodes to inject current into the subject and measure the drop in voltage across the subject using a high input impedance voltage measuring circuit.

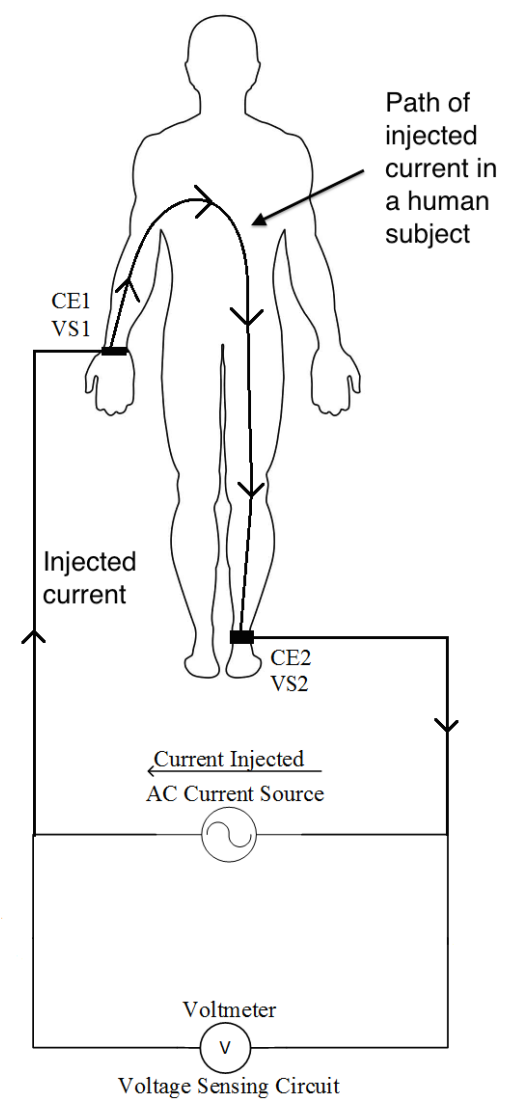

Figure 2.1: Bi-polar method injects current into the subject and senses voltage across the subject using the same electrodes. The AC current source in the picture injects current into the human subject through the electrodes CE1 (VS1) and CE2 (VS2). Voltage drop across the subject is sensed and shown by a voltmeter using the same electrodes VS1 (CE1) and VS2 (CE2). 
The human subject, current source circuit and the voltage measuring circuit setup shown in the figure 2.1 is simplified and shown in the figure 2.2. The bioimpedance of the human subject is indicated by a resistor of unknown value which is the desired quantity. An AC current of known phase and amplitude enters the resistor through the node CE1 and leaves the subject through the node CE2. The voltage across the resistor is the potential difference between the nodes VS1 and VS2. In case of the bi-polar method, the nodes VS1 and CE1, and, the nodes VS2 and CE2 are the same electrodes.

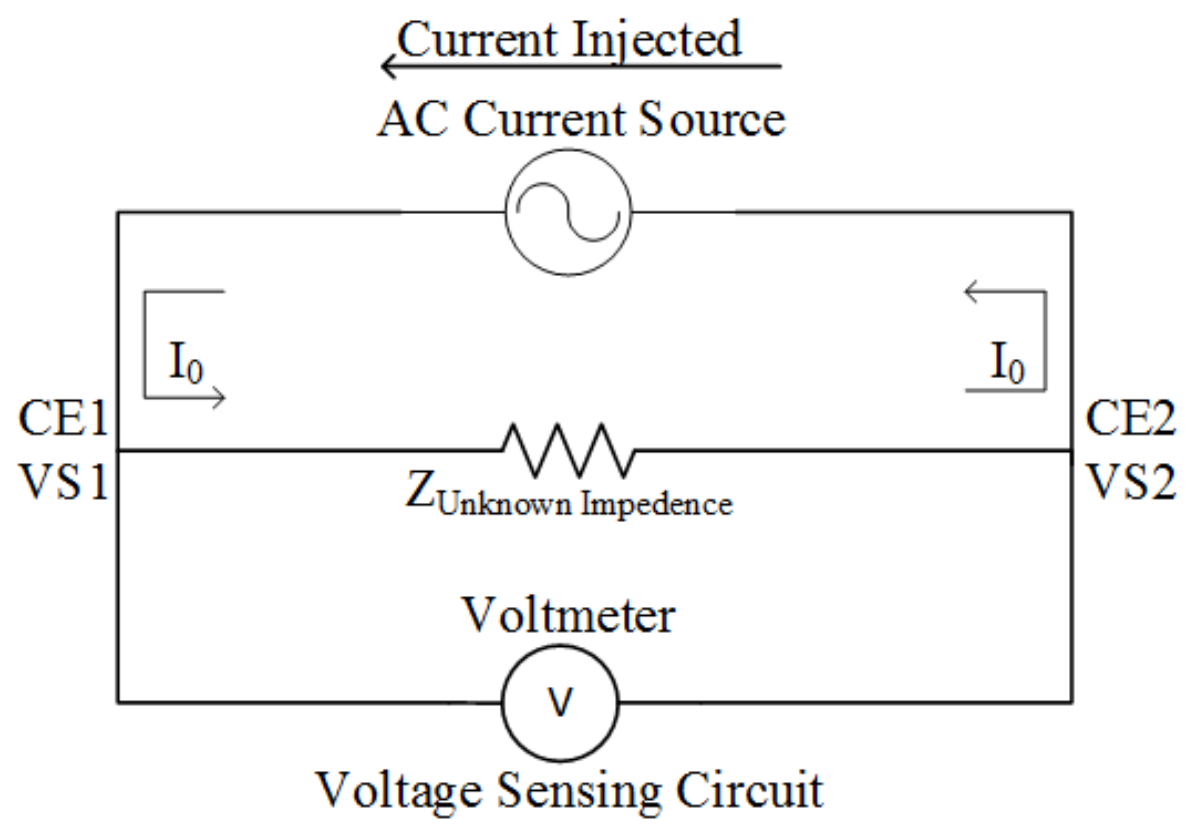

Figure 2.2: This figure shows the electrical model of the bi-polar method. An alternating current is injected and returned into the unknown impedance through the nodes CE1 and CE2 respectively. Voltage across the unknown impedance is measured by a voltage measuring circuit across the nodes VS1 and VS2.

If a known alternating current of RMS value $I_{O, R M S}$ is injected into the resistor of the value $Z_{\text {UnknownImpedance }}$ and a voltage of $\Delta V_{R M S}$ is recorded by the voltmeter, 
then the unknown impedance value can be calculated by

$$
Z_{\text {UnknownImpedance }}=\frac{\Delta V_{R M S}}{I_{O, R M S}}=\frac{\left(V_{S 1}-V_{S 2}\right)_{R M S}}{I_{O, R M S}}
$$

The drawbacks of the bi-polar method are:

(a) Using the same pair of electrodes utilized in injecting current into the subject to measure voltage drop across the subject makes the bioimpedance system vulnerable to the electrode-skin interface impedance and skin impedance interferences.

While the electrode-skin interface impedance depends of the frequency of operations, and at high frequencies it is still comparable to bioimpedance.

Under normal conditions for the skin, the skin impedance is several orders higher in magnitude compared to bioimpedance and it is very difficult to extract the value of bioimpedance from the recorded voltage measurements.

(b) Bi-polar method causes the voltage acquisition circuit to become vulnerable to impedance mismatch.

\section{Tetra-polar Method}

Tetra-polar method (also known as the four electrode technique) of current injection and voltage measuring techniques employs two different pairs of electrodes to inject current into the subject and measure the voltage across the subject. In this case, voltage measuring electrodes are placed between the current injecting electrodes. 


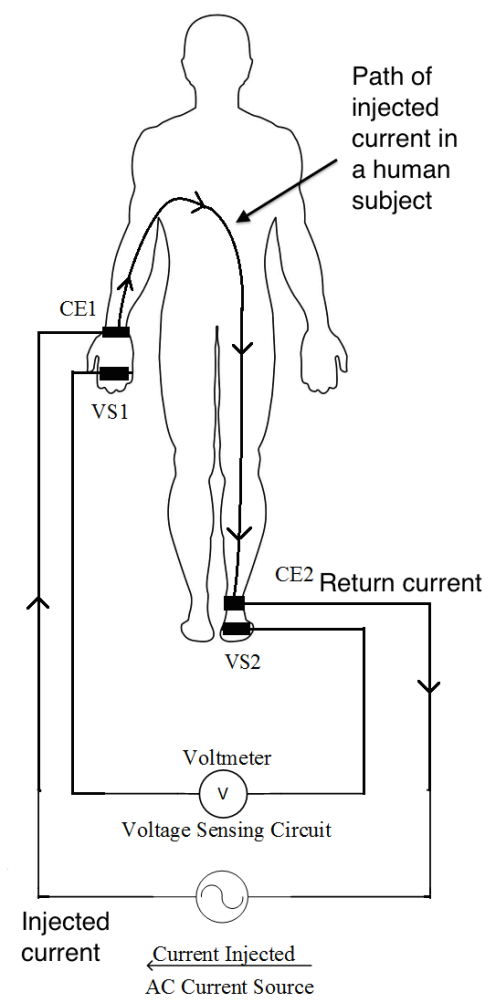

Figure 2.3: Tetra-polar method injects current into the subject and senses voltage across the subject using the different pairs of electrodes. The AC current source in the picture injects alternating current into the human subject through the electrodes CE1 and CE2. Voltage drop across the subject is sensed and shown by a voltmeter using a different pair of eletrodes indicated by electrodes VS1 and VS2. 
The human subject, current source circuit, and the voltage measuring circuit setup, shown in the figure 2.3, is simplified and shown in the figure 2.4. The bioimpedance of the human subject is indicated by a resistor of unknown value which is the desired quantity. An AC current of known phase and amplitude enters the resistor through the node $\mathrm{CE} 1$ and leaves the subject through the node CE2. The voltage across the resistor is the potential difference between the nodes VS1 and VS2. In the case of tetra-polar method, contrary to the bipolar method, the current injection electrodes CE1 and CE2 are different from the voltage measuring electrodes VS1 and VS2. This ensures that the electrodeto-skin interface interference is absent when the voltage is measured.

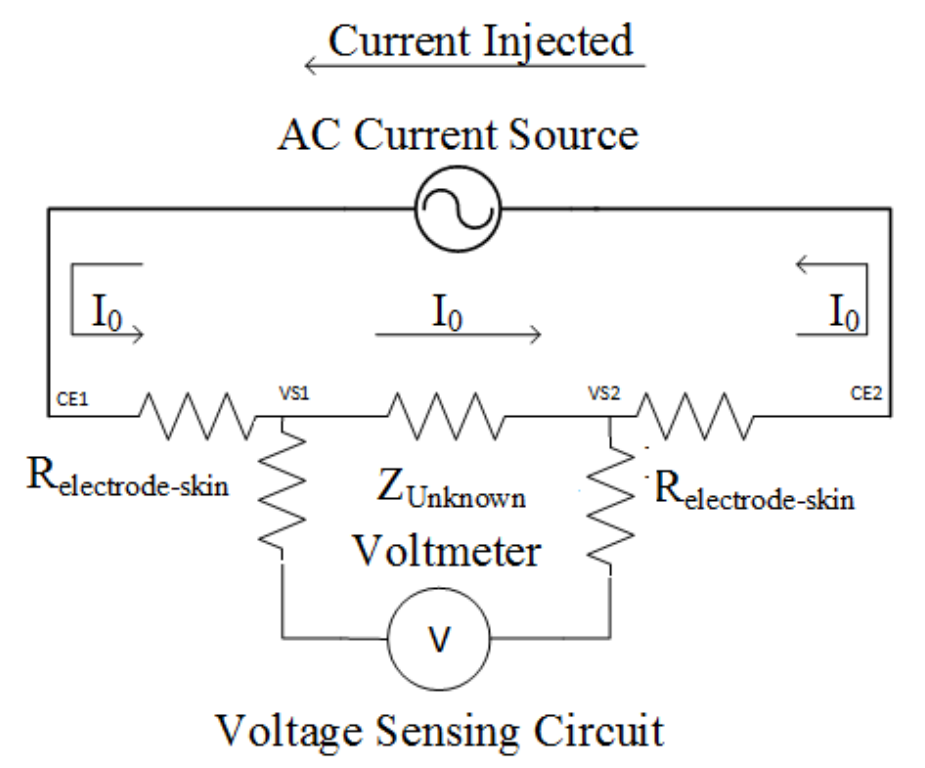

Figure 2.4: This figure shows the electrical model of the tetra-polar method. An alternating current is injected and returned into the unknown impedance through the node CE1 and CE2 respectively. Voltage across the unknown impedance is sensed and shown by the voltameter across the nodes VS1 and VS2.

If a known alternating current of RMS value $I_{O, R M S}$ is injected into the resistor of 
the value $Z_{\text {UnknownImpedance }}$ and a voltage of $\Delta V_{R M S}$ is recorded by the voltmeter, then the unknown impedance value can be calculated by

$$
Z_{\text {UnknownImpedance }}=\frac{\Delta V_{R M S}}{I_{O, R M S}}=\frac{\left(V_{S 1}-V_{S 2}\right)_{R M S}}{I_{O, R M S}}
$$

Tetra-polar technique is preferred over bi-polar technique because:

a. Interference of skin impedance is eliminated by the voltage measuring circuit by measuring only the voltage across the unknown impedance.

b. Tetra-polar technique is insensitive to changes in electrode-subject impedance of the current-injecting electrode.

c. Tetra-polar technique is also insensitive to electrode artifacts.

d. Tetra-polar technique minimizes the effect of input impedance mismatch at the front end of the voltage measuring circuit.

\subsubsection{Practical Considerations}

Several things that need to be considered while implementing the system to find bioimpedance are:

a. Positioning of the subject [3]:

The path taken by any injected current in the body is the least resistance path and is also determined by the point of injection.

When a human being is subjected to bi-polar or tetra-polar method as seen in the figues 2.1 and 2.3 respectively, the current injected takes the following path:

In the figure 2.3, assuming that the current is injected through the electrode CE1 and leaves through CE2, the current injected starts at the point of injection, the right-hand wrist, traverses up through the right arm, then enters the trunk which 
is a comparitively low resistance region, then enters the left leg and leaves the body through the left ankle.

This implies that the arms and legs have to be free of contact with one another and also have to free be from contact with any other par of the body. If due care is not taken in this regards, the observed voltages would result in injected current taking a different path other than the desired path. This leads of incorrect voltage measurements and thereby yielding incorrect values for bioimpedance.

b. Positioning of the electrodes:

The current injected by the current source through the electrodes is not uniformly distributed in the region close to electrode-skin interface (point of contact between electrodes and the skin). The non-uniform distribution of current at the point of contact necessitates that the voltage measuring electrodes be placed at a distance away from the current injecting electrodes. This is to ensure that the current is uniformly distributed across a subject and registers proper voltage drop. If the voltage measuring electrodes are placed close to current injecting electrodes, the drop in voltage measured would be high due to high current density close to electrode-skin interface. Also, the voltage measuring electrodes cannot be placed too far away from the current injecting electrodes as it leads to sensing lower voltage levels, thereby resulting in low bioimpedance values.

\subsection{Proposed Bioimpedance System Architecture}

Bioimpedance of a subject is measured by analyzing the response of the Subject Under Test (SUT) to a galvanostatic excitation or a potentiostatic excitation. Potentiostatic excitation of the SUT involves measuring the current response to an 
applied voltage excitation signal while, galvanostatic excitation involves injecting $\mathrm{AC}$ current into the subject and measuring the voltage the subject generates. Galvanostatic excitation is preferred to potentiostatic excitation because:

a. Potentiostatic excitation involves piercing electrodes into the body (invasive method) for reliable and accurate results.

b. If the potentiostatic excitation electrodes are too close, a small difference in potential could generate a very strong electric field that is fatal for a living biological subject [4].

c. In the case of non-invasive potentiostatic excitation of the biological subject, skin impedance interferes with the acquisition of the current response of the subject.

d. The impedance of a tissue is dependent on the condition of the tissue, temperature and point of contact of electrodes on the body. Potentiostatic excitation could result in large currents flow in the body if tissue impedance is too low(which could be an ensuant of one factor or another). This could be fatal for the subject.

\subsubsection{Architecture Overview}

Bioimpedance system that is designed for the thesis involves galvanostatic excitation of a biological subject, i.e. injecting an alternating current of a known value into the subject and measuring the voltage response of the subject.

An alternating current source injects into the subject an $\mathrm{AC}$ current of a known amplitude and frequency. The subject is typically a biological subject. In the figure 2.5, the arrows in and out of the subject indicate the direction of the injected current and the return current respectively. 


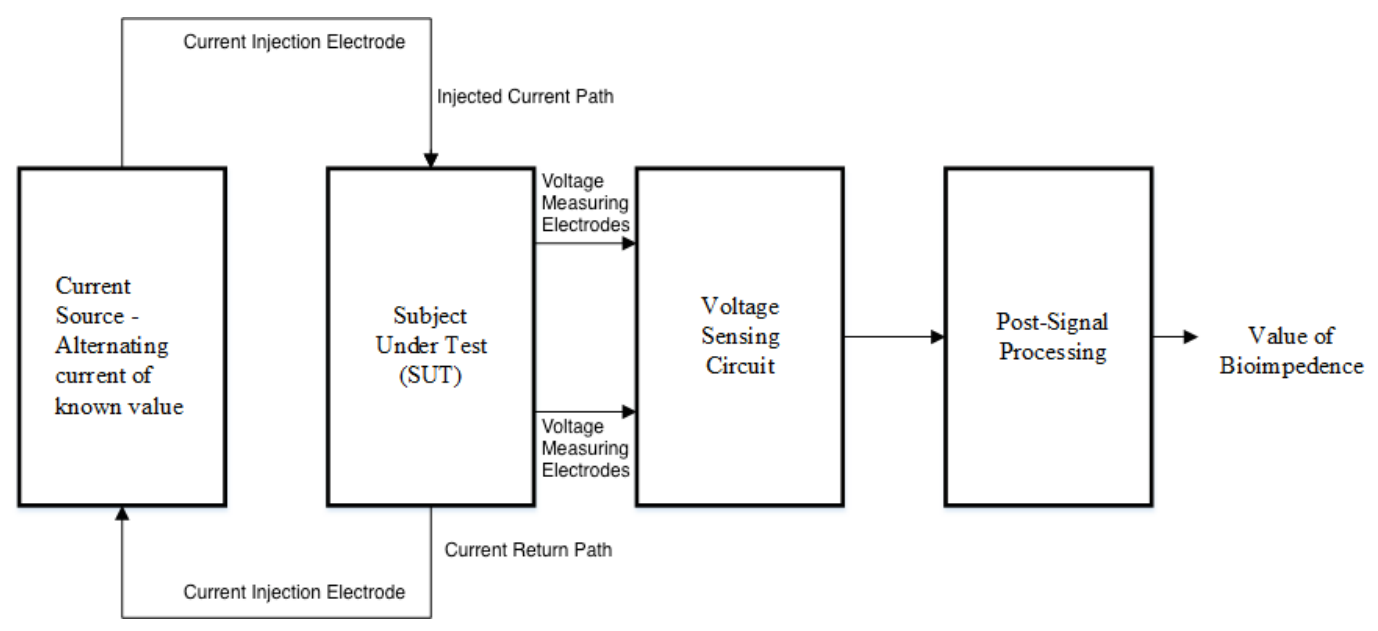

Figure 2.5: The figure illustrates the bioimpedance measurement system based on galvanostatic excitation method of bioimpedance. The current source injects current into the subject under test (arrows indicating the direction of injected current and return current). The acquired voltage reponse is provided as the input to a post processing circuit to determine bioimpedance.

The voltage response produced by the subject is acquired using a voltage measuring circuit such as an op-amp configured as a difference amplifier or an instrumentation amplifier. Often, the front end of the voltage measuring circuit is a low pass filter or a high pass filter depending on the application and frequencies of interest.

The voltage output of the voltage measuring circuit is later processed by using a post processing circuit.

Mathematically, the value of an unknown impedance of a subject that is subjected to galvanostatic excitation is equal to the ratio of RMS value of the $\mathrm{AC}$ voltage response of the subject $\left(V_{R M S}\right)$ to the RMS value of $\mathrm{AC}$ current $\left(I_{R M S}\right)$ that is injected into the subject.

$$
Z_{\text {unknown }}=\frac{V_{R M S}}{I_{R M S}}
$$




\subsection{Proposed Bioimpedance System Specifications}

This section deals with the design specifications as well as various parameters of the bioimpedance system and presents the rationale behind their values.

The International Electrotechnical Commission (IEC) published a series of technical standards, IEC60601, standardizing the medical equipment and general requirements for performance and safety of a subject. Though some deviation exists from the standard depending on the country, the IEC60601-1 is widely accepted and adhered to.

\subsubsection{Current Injection}

According to IEC60601, the maximum DC current that can be injected into the human body under normal conditions cannot exceed 10uA.

The maximum AC current injected into the human subject is a function of frequency and the relationship between the injected current and the frequency is as follow:

$$
I_{A C(\max )}=\frac{F_{E} \cdot 10 u A_{R M S}}{1000 H z}
$$

where $F_{E}$ is the excitation frequency, $I_{A C}$ is the maximum RMS current for a particular excitation frequency.

The above relationship between the maximum current and excitation frequency is valid only for frequencies greater than $1 \mathrm{kHz}$. For frequencies lower than or equal to $1 \mathrm{kHz}$ the maximum current allowed is $10 \mu A_{R M S}$. The frequency of operation depends on the requirements and technique followed in determining the bioimpedance. 
Whole body impedance, bioimpedance of the whole body, can be determined by injecting current at a single frequency or multiple frequencies. Although there is no consensus on the magnitude of frequency (or frequencies) of operation among the researchers, a frequency of $50 \mathrm{kHz}$ is widely accepted for single frequency technique and the set of frequencies in case of multi-frequency technique may vary depending on the requirement and other parameters.

This thesis aims at determining bioimpedance by injecting current of different magnitude into the body at multiple frequencies and determine bioimpedance of a biological subject. The maximum frequency used will be $50 \mathrm{kHz}$.

The current source circuit that is designed has to be able to inject currents of different magnitudes at different frequencies. Ideally, the desired output impedance of a current source is infinite. This is to ensure that the output current is constant, regardless of the load impedance and the load voltage. Practically, this has to be a very high value. For the thesis, a modified Howland current source, an op-amp based current source circuit, is designed to inject current into an SUT. The output impedance of a modified Howland current source depends on the gain of the op-amp it is based on, and high the gain of the op-amp higher is the output impedance.

For a $50 \mathrm{kHz}$ frequency, the maximum current that can be injected into a biological subject can be calculated from equation 2.5 and is given by

$$
I_{A C(\max )}=\frac{F_{E} \cdot 10 u A_{R M S}}{1000 H z}=\frac{50 K \cdot 10 \mu}{1 K}=500 \mu A_{R M S}
$$

The value of current obtained from the above equation 2.6 is RMS current mag- 
nitude, hence the peak value or the amplitude of the injected current is

$$
I_{A C(\text { peak })}=\frac{I_{A C(R M S)}}{0.707}=707.2 \mu A \quad 700 \mu A
$$

\subsubsection{Voltage Measurement}

The value of bioimpedance of a human body is dependent upon many factors such as its health conditions, ethnicity, etc. Typically, the value for whole body impedance ranges from $500 \Omega$ to $700 \Omega$. Sometimes, depending on the requirement and purpose, bioimpedance of a part of the human body is needed and determining the bioimpedance of segment of the body is termed segmental bioimpedance. The bioimpedance of a part of a human body is lower than the whole body impedance for obvious reasons. For example, the trunk of a human body constitutes about 8 percent of the total body impedance. Therefore, if the whole body impedance is $500 \Omega$, then the bioimpedance of the trunk of the body is

$$
Z_{\text {Trunk }}=0.08 \cdot(500)=40 \Omega
$$

For a frequency of $1 \mathrm{kHz}$, the maximum current that can be injected into a human

body is $1 \mu A$. If segmental bioimpedance is performed to find bioimpedance of the trunk, the voltage signal produced is from equation 2.5 if the impedance of the trunk is $40 \Omega$

$$
\Delta V_{\text {Trunk }}=40 \Omega \cdot(1 \mu A)=0.40 \mathrm{mV}
$$


The obtained $\Delta V_{\text {Trunk }}$ value is so low that the noise in the circuit could be a detracting factor when measuring it. This problem can be overcome by injecting higher currents while increasing the frequency or operating the voltage measuring circuit at a gain higher than unity and later taking care of the gain factor during the post-processing of the signal. Regardless of the method chosen to overcome the issues with low impedance, it is imperative that the voltage measuring circuit requires a very high common mode rejection ratio (CMRR) and be able to operate at multiple gains.

For the thesis, a 2-stage op-amp based instrumentation amplifier is designed to acquire the voltage signal produced by the unknown impedance.

Assuming the unknown bioimpedance is $500 \Omega$ and the peak current injected is $700 \mu A$, the peak voltage of the voltage signal produced is

$$
\Delta V_{\text {peak }}=700 \mu \cdot(500 \Omega)=350 \mathrm{mV}
$$

A voltage signal with an amplitude of $350 \mathrm{mV}$ is a large signal. Though it is not always the case that the current injected is as high as $700 \mu \mathrm{A}$ for a $50 \mathrm{kHz}$ signal, it befits to have a large Input Common Mode Range (ICMR) op-amps at the front end of the instrumentation amplifier.

If the instrumentation amplifier is to operate at a gain higher than unity, a wide output voltage swing for the designed instrumentation amplifier is suitable for a large voltage input signal.

As discussed before, a high CMRR voltage acquisition and measuring circuit is suitable for the application and a high CMRR op-amp helps the case.

Arriving at the power consumption for the system, it is preferable to have a 
portable bioimpedance system which can derive its power from a battery and serve the need for as long as possible. A very low power circuit with a small form factor make it possible to realize the portable bioimpedance system that is sought, and as such,a design with power consumption less than $1 \mathrm{~mW}$ is desired.

The preceding discussion leads us to the design specifications shown in the following tables for the op-amp and op-amp based current source and the instrumentation amplifier.

The desired high CMRR, low power op-amp specifications are:

\begin{tabular}{|c|c|}
\hline Specification & Target \\
\hline Differential gain $\left(A_{d}\right)$ & $>70 \mathrm{~dB}$ \\
\hline Common mode rejection ratio $(\mathrm{CMRR})$ & $>85 \mathrm{~dB}$ \\
\hline Unity Gain Bandwidth & $>100 \mathrm{kHz}$ \\
\hline Input common mode range $(\mathrm{ICMR})$ & $>200 \mathrm{mV}$ and $<1100 \mathrm{mV}$ \\
\hline Output voltage swing & $>500 \mathrm{mV}$ \\
\hline Power consumption & $<200 \mu W$ \\
\hline
\end{tabular}

Table 2.1: Op-amp design specifications 
Desirable current source circuit specifications are:

\begin{tabular}{|c|c|}
\hline Specificiation & Target \\
\hline Output impedance $\left(Z_{\text {OUT }}\right)$ & $>100 \mathrm{~K} \Omega$ \\
\hline Unity Gain Bandwidth $(\mathrm{UGBW})$ & $>100 \mathrm{kHz}$ \\
\hline Output voltage swing & $>500 \mathrm{mV}$ \\
\hline Power consumption & $<200 \mu \mathrm{W}$ \\
\hline
\end{tabular}

Table 2.2: Current source design specifications

Desirable specifications for the voltage measuring circuit are:

\begin{tabular}{|c|c|}
\hline Specificiation & Target \\
\hline Common mode rejection ratio & $>100 \mathrm{~dB}$ \\
\hline Unity Gain Bandwidth & $>100 \mathrm{kHz}$ \\
\hline Input common mode range & $>200 \mathrm{mV}$ and $<1100 \mathrm{mV}$ \\
\hline Output voltage swing & $>500 \mathrm{mV}$ \\
\hline Power consumption & $<600 \mu \mathrm{W}$ \\
\hline
\end{tabular}

Table 2.3: Voltage measuring circuit design specifications 


\subsection{Hardware Modules of the Bioimpedance Sys-}

\section{tem}

This section deals with individual blocks of the system, various circuits and final design specifications for the individual blocks.

The individual blocks of the system are:

a. Current source circuit

b. Voltage measuring circuit

c. Post signal processing circuit.

\subsubsection{Current Source}

In short, a current source circuit is an electronic circuit which produces current output by either sourcing current or sinking current from a load and in this case the biological subject. Ideally, the desired output impedance of a current source is infinite and practically a very high value in order to maintain the output current from the current source invariable, irrespective of the voltage across the load.

Depending on the input signal, there are two types of current sources. The output current of a current source is controlled either by a voltage input signal or a current input signal.

A current controlled current source is a circuit whose output current is a function of input current. Examples of current controlled current sources include current mirror, cascode current mirror, current conveyor, etc.

A current mirror is a simple current source circuit frequenlty used in op-amps for biasing purposes. A basic current mirror is a two transistor circuit whose current 
output is controlled by a current input. Depending on the magnitude of widths and lengths of the transistors, the output current can be manipulated. 


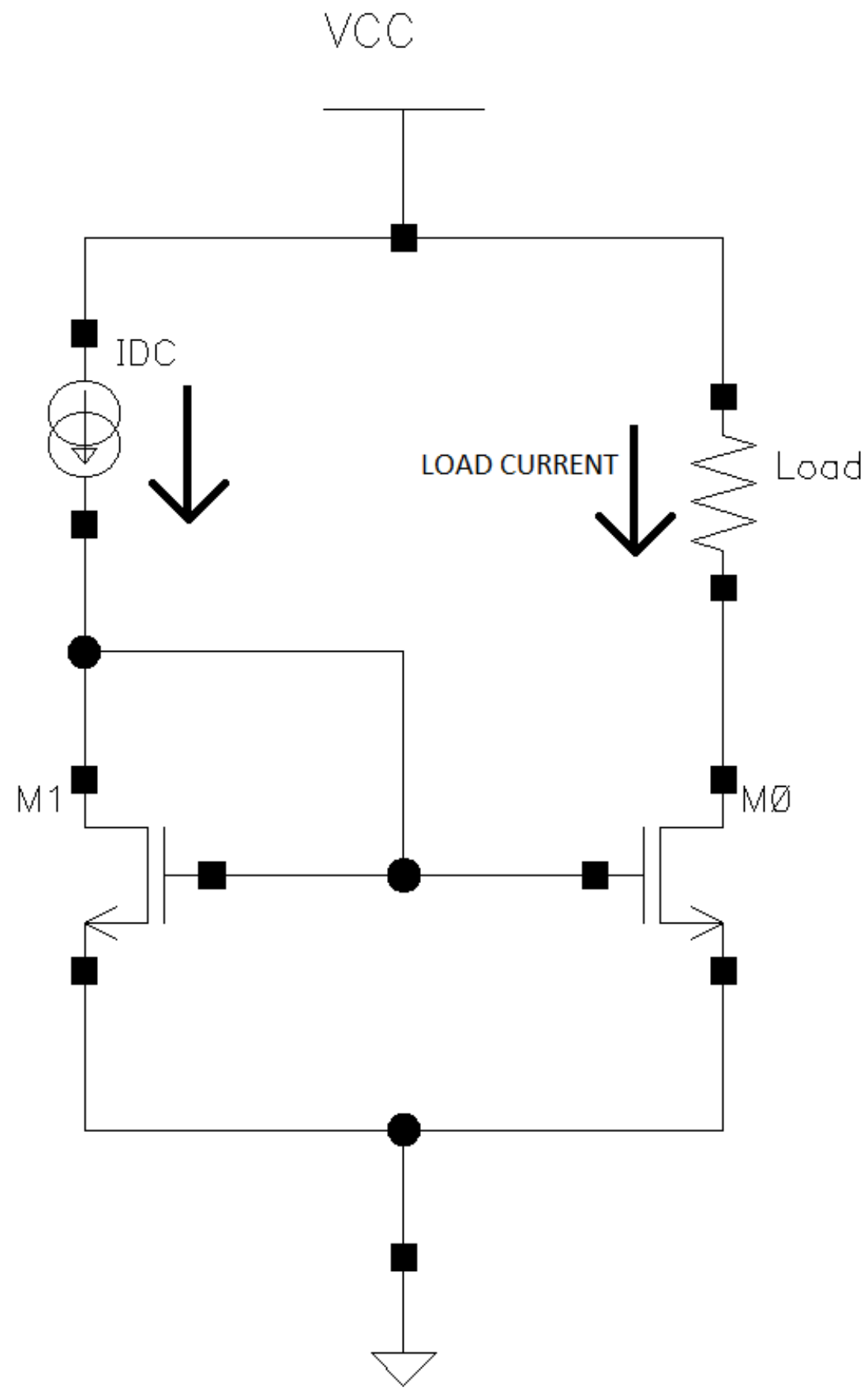

Figure 2.6: The current that is injected into the diode-connected M1 transistor causes the same amount of current to be flown in M0 transistor if the width and length dimensions of the transistors are the same. The output impedance of the circuit is the output impedance of M0. 
While the simplicity and low layout area constraints of a basic current mirror are certainly advantageous, the circuit suffers from low output impedance and poor performance in noisy environments when compared with other current sources. A cascode current mirror is another current controlled current source that can be best described as a stack up of basic current mirrors. Though a cascode current mirror is an improvement over the basic current mirror considering a relatively higher output impedance, its performance is poor in noisy environments. Also, trying to maintain the stacked transistors in saturation reduces output voltage swing significantly. 


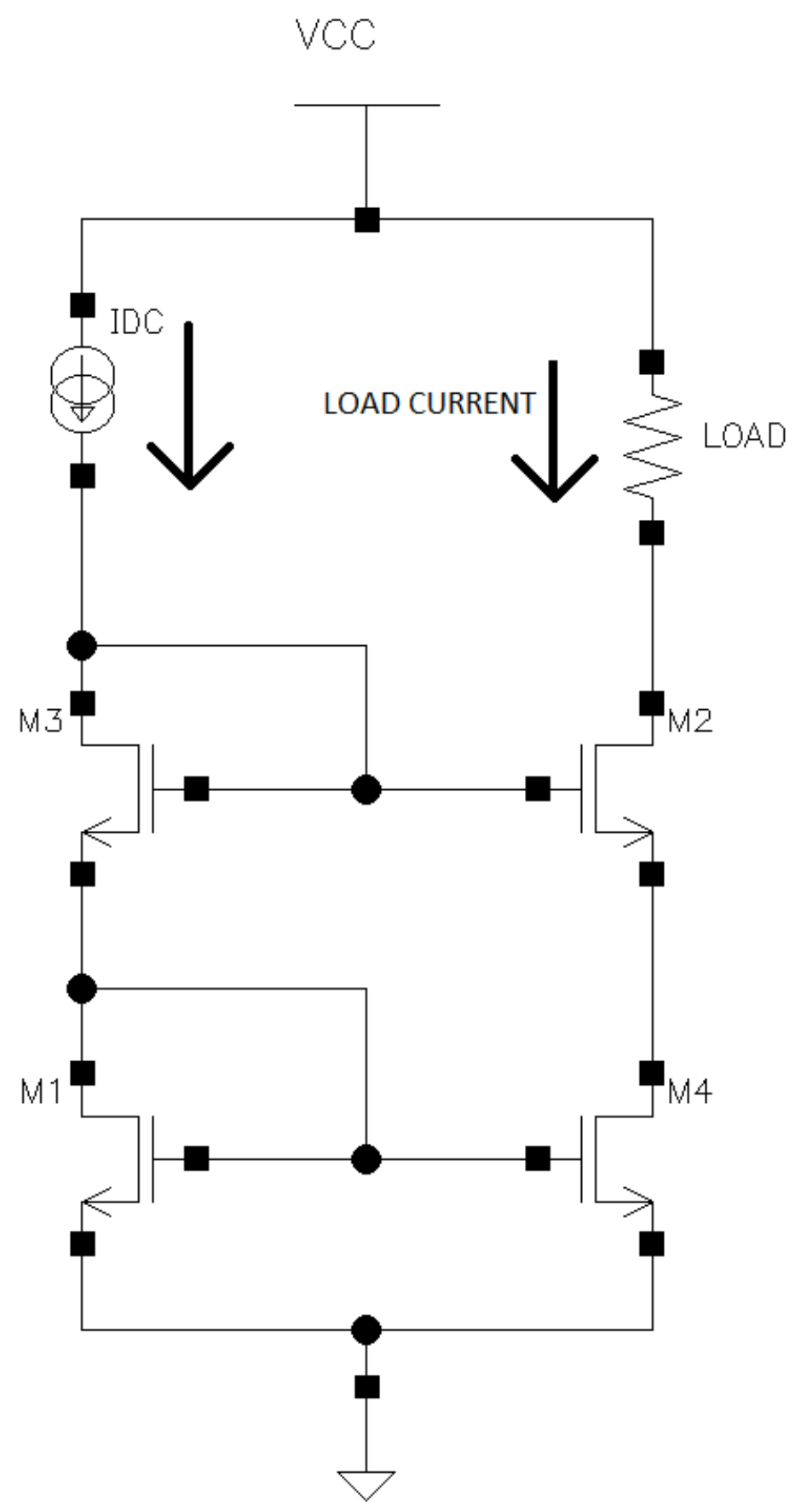

Figure 2.7: The current that is injected into the drain of M3 is the same current that flows in M1 since they are in series. For identical M2 and M3, M4 and M1 the current is the same as the current injected in M3. The output impedance of M4 and M2 are in series, resulting in an increased output impedance of the cascode current source. 
Current conveyor is another current controlled current source.

The explanation of the current conveyor current source shown in figure 2.8 ex-

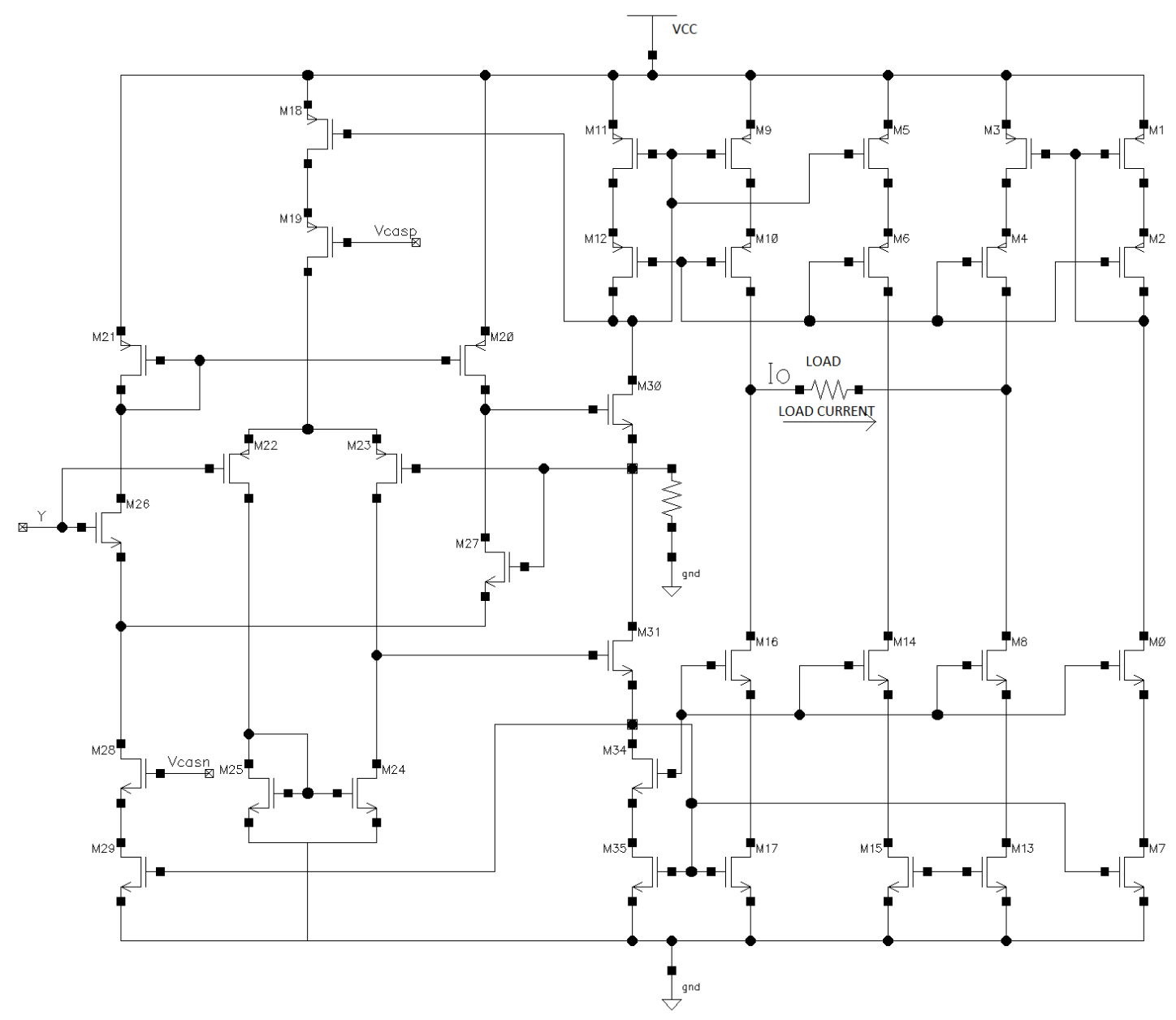

Figure 2.8: The current conveyor current source

ceeds the scope of this thesis, however the pros and cons of the circuit can be discussed.

The output impedance performance and current sourcing capability in noisy environments of a current conveyor is a significant improvement when compared to the current mirror circuit, but, the circuit fares poorly in terms of design complexity and requires precise matching of the transistors.

Though not a directly visible issue, voltage sources are inexpensive and easily 
available compared to relatively pricey precise current sources.

A voltage controlled current source is a current source whose output current is a function of an input voltage.

Though a MOSFET, P-channel or N-channel MOSFET, is the simplest voltage controlled current output device when operated in saturation, it is seldom used as a current source because of very low output impedance and its vulnerability to variations in process, temperature and noise.

An op-amp based voltage controlled current source is a significant improvement when compared to a MOSFET. A high common mode rejection ratio rejects common mode noise at the input and with a decent design it is relatively resistant towards process and temperature variations, though at the cost of layout area and power consumption.

The circuit in figure 2.9 displays a voltage controlled current source.

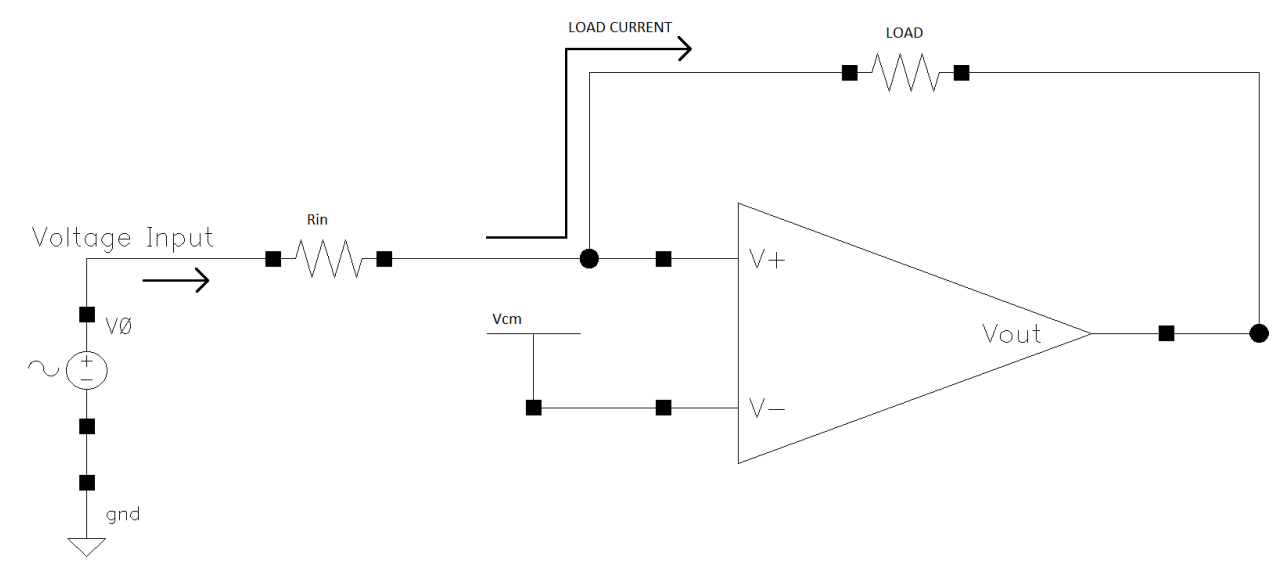

Figure 2.9: A Simple op-amp based voltage controlled current source 
In the figure 2.9, the voltage of inverting and non-inverting terminals of the op-amp is the common mode DC that is applied at the inverting terminal. The AC input signal that is applied at the $R_{\text {in }}$ resistor causes a voltage difference across it. The difference in voltage leads to current in the resistor. As no current flows into an operational amplifier, the current flows into the load. Thus, current injected into load is voltage controlled and the current source is a voltage controlled current source.

The shortcoming of the circuit is that the current injected is limited by the value of input resistance. For higher currents, the value of the input resistance has to be decreased. However, decreased in the resistance of $R_{\text {in }}$ marks reduction in input impedance of the op-amp, causing the operational amplifier to become vulnerable to noise and mismatch. Also, since the load is connected to $R_{i n}$, reduced resistance of $R_{\text {in }}$ reduces the output impedance of the current source which is the load's point of connection. Regardless of the shortcoming of the circuit, the voltage controlled current source is relatively easy to design, reduced in complexity and can be improved to perform better.

The aforementioned discussion of current controlled current sources and voltage controlled current sources provide the rationale in choosing a voltage controlled current source over a current controlled current source. This thesis employs mirrored modified Howland current source, an op-amp based current source and a variant of basic Howland current developed by Brad Howland, as the current source to inject a current of known amplitude and phase into the SUT.

\section{Basic Howland Current Source}

The Howland current source circuit is an op-amp based current source invented 
by Brad Howland at Massachusetts Institute of Technology (MIT) in 1963.

The Howland current source seen in the figure 2.10 clearly shows a positive feed-

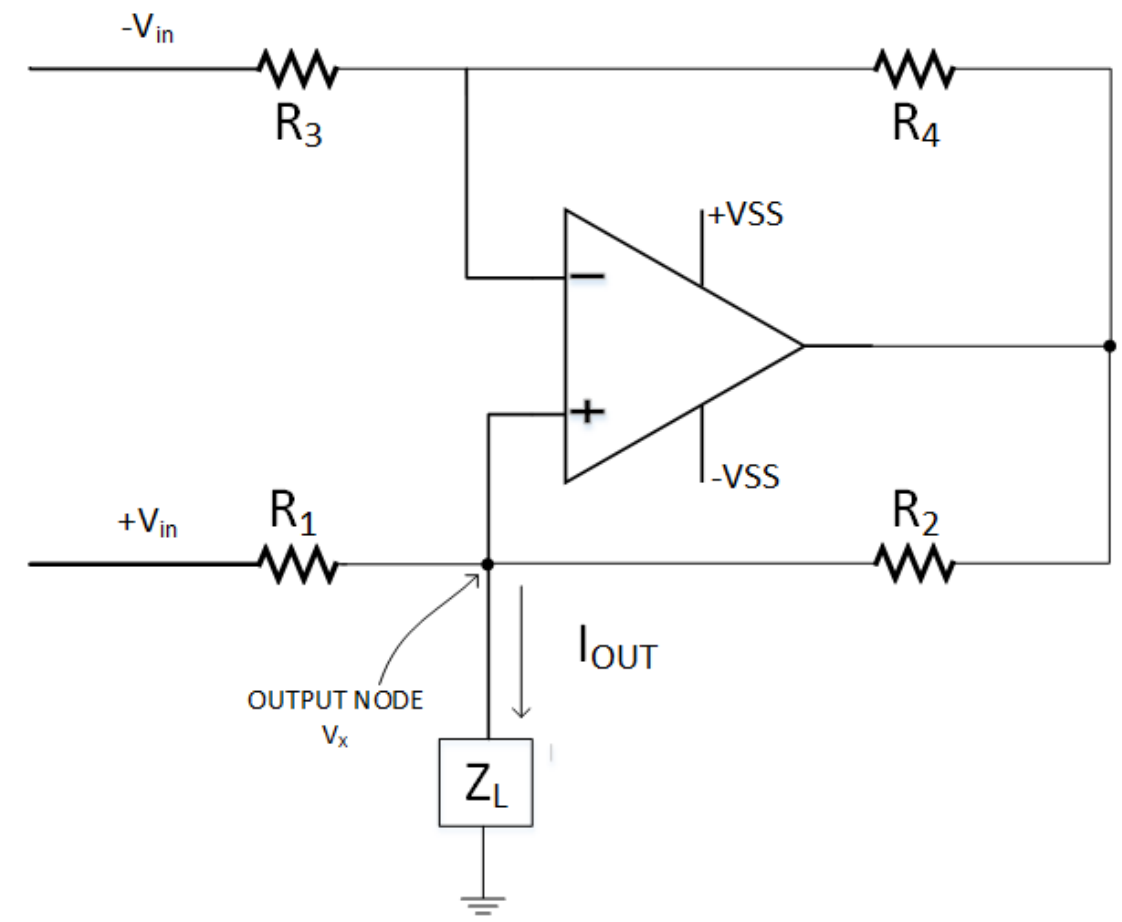

Figure 2.10: The figure shows a basic Howland current source [5]. The circuit works as a current source by bootstrapping load current in the positive feedback path if the resistor ratios are equal, i.e. $R_{4} / R_{3}=R_{2} / R_{1}$.

back to the non-inverting input terminal which provides a bootstrap effect and keeps the load current in the load constant, thereby acting as a current source.

In the figure $2.10 Z_{L}$ represents the load resistance and the load current is $I_{\text {OUT }}$. The resistors $R_{4}$ and $R_{2}$ are feedback resistors in negative and positive feedback paths respectively. The resistors $R_{3}$ and $R_{1}$ are input resistors.

Assume that the current in the resistors $R_{x}$ is $I_{x}$.

By applying KCL at the inverting terminal of the op-amp,

$$
I_{3}=I_{4}
$$


because there is no current flowing into the inverting terminal of the op-amp.

By applying KCL at the non-inverting terminal of the op-amp,

$$
I_{1}=I_{4}+I_{O U T}
$$

because there is no current flowing into the non-inverting terminal of the op-amp. Transforming the current equations into voltage equations, from equation 2.11 the voltage equation obtained is

$$
\frac{-V_{i n}-V_{x}}{R_{3}}=\frac{V_{x}-V_{o}}{R_{4}}
$$

From equation 2.12 , the voltage equation obtained is

$$
\frac{+V_{i n}-V_{x}}{R_{1}}=\frac{V_{x}-V_{o}}{R_{2}}+I_{O U T}
$$

To simplify the equation, assume $\mathrm{a}=V_{x}-V_{o}$. The equations 2.13 and 2.14 simplify to

$$
\frac{-V_{i n}-V_{x}}{R_{3}}=\frac{a}{R_{4}}
$$

and

$$
\frac{+V_{i n}-V_{x}}{R_{1}}=\frac{a}{R_{2}}+I_{O U T}
$$

respectively.

Assuming,

$$
\frac{R_{2}}{R_{1}}=\frac{R_{4}}{R_{3}}
$$


the equation 2.15 can be re-written as,

$$
\frac{-V_{i n}-V_{x}}{R_{1}}=\frac{a}{R_{2}}
$$

Solving for $I_{O U T}$ in equation 2.16,

$$
I_{\text {OUT }}=\frac{+V_{i n}-V_{x}}{R_{1}}-\frac{-V_{i n}-V_{x}}{R_{1}}
$$

Simplifying the equation further,

$$
I_{O U T}=\frac{+V_{i n}--V_{i n}}{R_{1}}
$$

Therefore, the output current or the load current is

$$
I_{\text {OUT }}=\frac{+V_{i n}--V_{i n}}{R_{1}}=\frac{V_{d i f f}}{R_{1}}
$$

assuming that the amplitude of the differential input is $V_{\text {diff }}$.

The essential conclusion that can be drawn from the load current equation (equation 2.21) is that the load current is independent of the output voltage of the operational amplifier and the load voltage. It is a function of the differential voltage input and the input resistor, $R_{1}$, iff the resistor ratio condition, shown in equation 2.17, is satisfied, and the load impedance far lower than the output impedance of the current source.

\section{Modified Howland Current Source}

The two main disadvantages of basic Howland current source are: 
i) In a basic Howland current source, increase in the input signal voltage amplitude to increase the output current of the current source is limited by the output voltage swing of the op-amp output, beyond which the op-amp output becomes non-linear.

ii) In the figure 2.10 and from equation 2.21, in order to increase the current drive of the current source the value of $R_{1}$ has to be reduced or the amplitude of the input signal has to be increased. While increasing the input has its limitations, the value of $R_{1}$ cannot be reduced beyond a certain limit. Reducing the resistance of $R_{1}$ reduces the output impedance of the current source and also results in an imbalance in the input impedance at the input terminals of the op-amp. The resultant imbalance could reduce the common mode rejection ratio of the circuit and the current source becomes vulnerable to noise and unstable.

iii) If the differential input voltage is large, the op-amp may become unstable because of the positive and negative feedback. The above mentioned disadvantages of the basic Howland current source prompts the designer to look into the modified Howland current source, aka improved Howland current source, which a variant of Howland current source. 


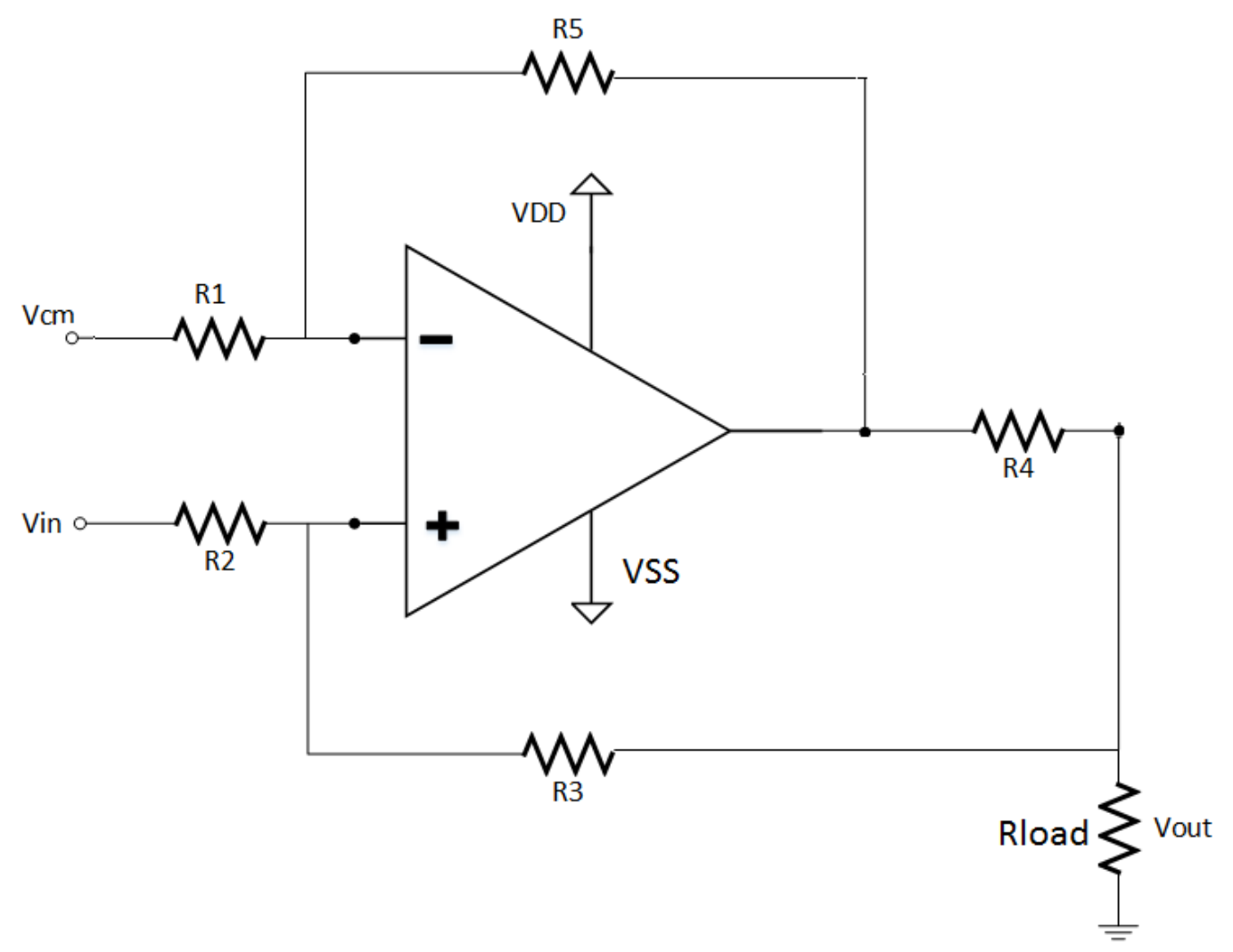

Figure 2.11: Figure shows a modified Howland current source [6], where the transconductance gain of the current source is set by $R_{4}$. Usually, the ratio is matched by increasing the value of $R_{2}$ such that resistor ratio is preserved 
The modified Howland current source is also a voltage controlled current source with a higher current gain and larger load voltage margin compared to the basic Howland current source.

A major difference between the basic and the modified Howland current source is the positive feedback resistor. In the case of modified Howland current source, the positive feedback resistor is split into two resistors which sum up to the original value of the resistor.

$$
R_{5}=R_{3}+R_{4}
$$

When a differential voltage input, $V_{i n}$, is applied to the positive input terminal through $R_{2}$ the resultant excess current passes through $R_{4}$ and through the load becoming the load current. The feedback current passing through $R_{4}$ and then through $R_{3}$ remains the same and is usually much lower than the load current. It is typically the case that the resistance of $R_{4}$ is much lower than $R_{3}$ so that the change in the resistance of $R_{2}$ remains small and the difference between $R_{2}$ and $R_{3}$ remains very small. This keeps the mismatch at the input very low. Mathematically, $R_{2}$ is

$$
R_{2}=R_{3}+R_{4}
$$

If

$$
R_{4}<<R_{3}
$$

then

$$
R_{2} \sim R_{1}
$$


If the difference between $R_{1}$ and $R_{2}$ is significant, the impedance mismatch at the input terminals can cause undesirable outcomes.

$$
\frac{R_{2}}{R_{3}}=\frac{R_{1}}{R_{5}+R_{4}}
$$

Therefore, modified Howland current source makes it possible to have a low valued $R_{4}$ and high feedback and input resistors to drive high output current without reducing input impedance.

In the figure 2.11,

$$
\begin{gathered}
R_{1}=R_{3}=R_{5}=R \\
R_{4}=r \\
R_{2}=R+r
\end{gathered}
$$

The load current, $I_{\text {Load }}$, passing through the load resistor is

$$
I_{\text {Load }}=\frac{V_{\text {in }}}{R_{4}}
$$

It is worth mentioning that the current equation 3.6 is valid as long as the opamp gain is high and the load impedance is very small compared to the output impedance of the current source.

\section{Mirrored Modified Howland Current Source}

Though an improvement over the basic Howland current source, the modified Howland current source is suitable only if the load is ground if the op-amp is a dual supply op-amp. For a single supply op-amp, i.e. where the lower power supply is $0 \mathrm{~V}$, the load has to be biased at mid-rail potential. This is relatively 
easy if the subject under test is an electrical subject. If the subject under test is a biological subject, it is easier to have a current source that could source current into a floating node than to deal with the complications of driving different parts of the subject to a potential while ensuring safety of the subject at the same time. This eventuates mirrored modified Howland current source.

Mirrored modified Howland current source circuit, shown in figure 2.12, consists of two modified Howland current sources. One of the modified Howland current sources sources the output current into the load and the other current source sinks current from the load. The magnitude of the output current from individual

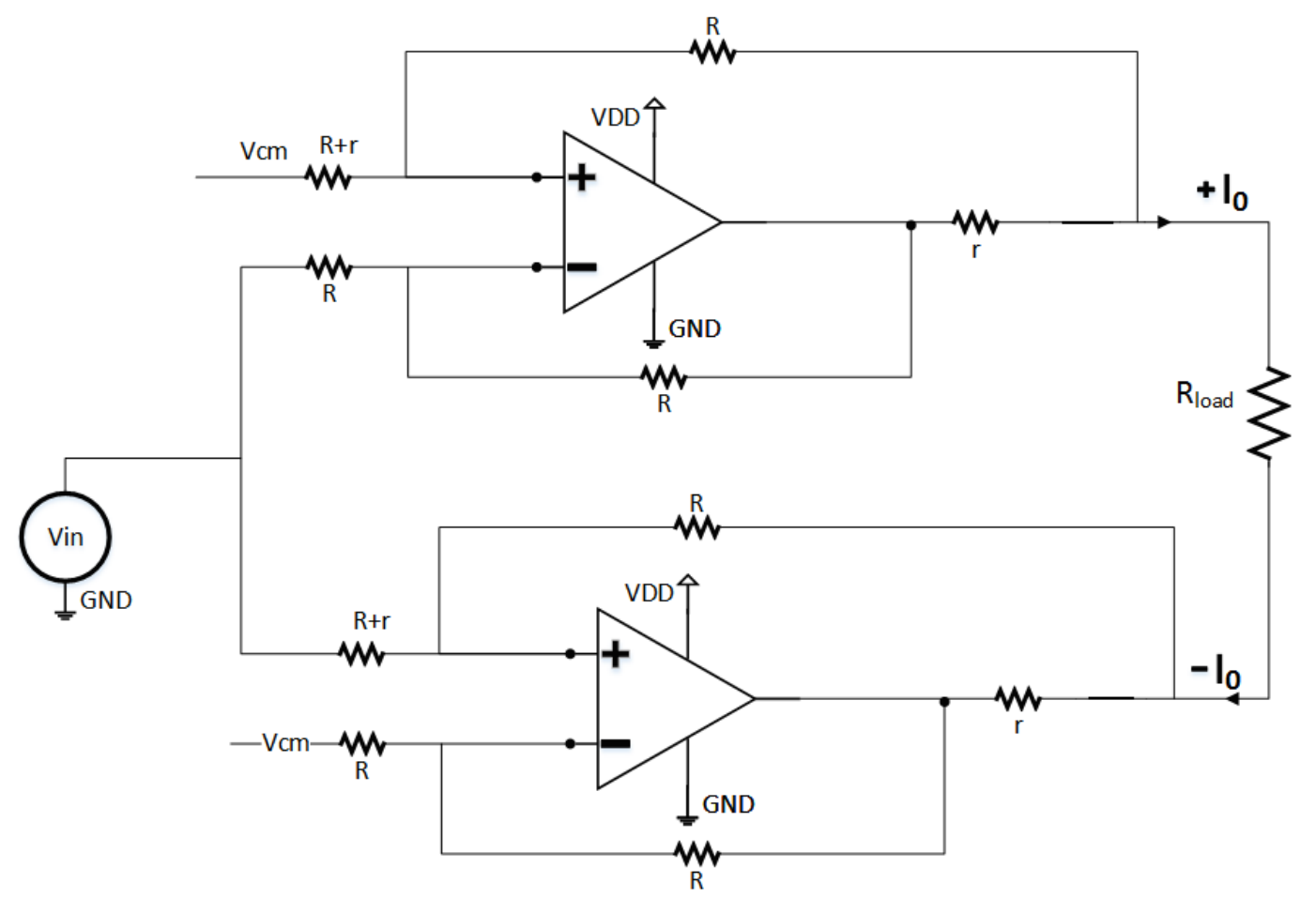

Figure 2.12: Figure shows a mirrored modified Howland current source, with a floating load $\left(R_{\text {Load }}\right)$ with two symmetrical modified Howland sources.

current sources is

$$
I_{o}=\frac{V_{i n}}{r}
$$


The gain and the output impedance of the individual current sources have to be high resulting in a high output impedance of the current source. The current source produces a current output that is purely dependent on the input signal and the gain setting resistor, regardless of the load connected to it.

Series connection between the modified Howland current sources and the output load necessitates matched transconductance gains of both the modified Howland current sources and matched resistors to match the branch gains of the individual current sources.

Apart from being able to support floating loads, the other advantages of mirrored modified Howland current source over the basic and modified Howland source are improved stability and increased output impedance.

\subsubsection{Voltage Measuring Circuit}

For the thesis, an instrumentation amplifier is designed to acquire the voltage response of the unknown impedance.

Well known ways to measure the voltage produced by an unknown impedance when current is injected into it is using an op-amp configured to work as a difference amplifier or an instrumentation amplifier.

A difference amplifier is a closed loop circuit which amplifies the difference between two input voltage signals and rejects the common mode of the input signals. An Instrumentation amplifier (in-amp) is a closed loop electronic circuit that has differential input and single ended output. The common mode voltage of the output voltage signal of the instrumentation amplifier is set by an input reference voltage. 


\section{Difference Amplifier}

A difference amplifier is one of the simplest op-amp based circuits that can measure and amplify (or attenuate) the difference between two voltage input signals. When the differential gain of the difference amplifier is set to 1 the circuit functions as a voltage acquisition and measuring circuit.

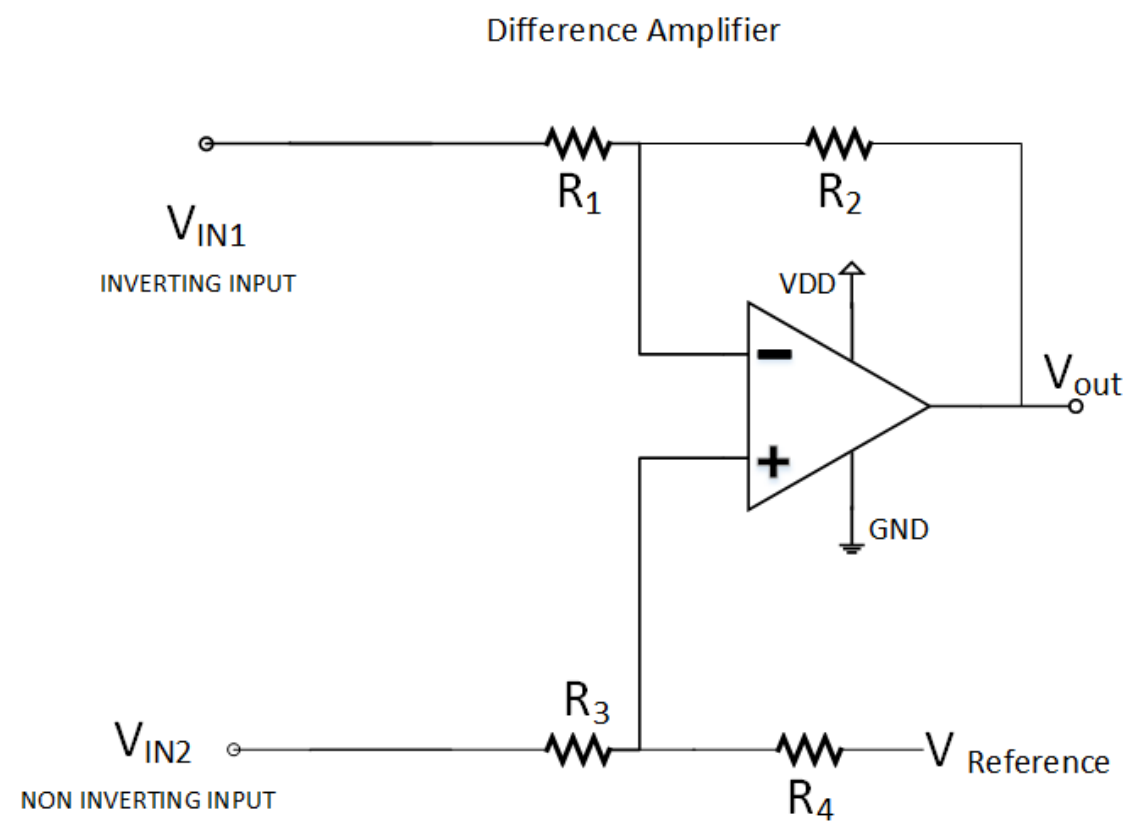

Figure 2.13: In the figure, an op-amp is configured as a difference amplifier. A difference amplifier measures (also attenuates or amplifies) the difference between its inverting and noninverting input signals depending on the gain set by the feedback and input resistors. The inverting input signal experiences a gain of $\left(-R_{2} / R_{1}\right)$ and the non-inverting input gain is $(1+$ $\left.R_{2} / R_{1}\right)$. The common mode voltage of the input signal is set by the reference input voltage.

In the figure 2.13 , if

$$
\begin{aligned}
& R_{1}=R_{3} \\
& R_{2}=R_{4}
\end{aligned}
$$

then

$$
V_{\text {out }}=\frac{R_{2}}{R_{1}} \cdot\left(V_{I N 2}-V_{I N 1}\right)
$$


Difference amplifier has two main issues that render them inefficient. They are:

a. The input impedance seen by the inverting and non-inverting input signals is relatively low and mismatch in resistors cause them to become unequal.

b. Mismatch between the resistor ratios leads to gain mismatch in both the branches of the op-amp and this also leads to degradation of CMRR.

\section{Instrumentation Amplifier}

The limitations of a difference amplifier can be overcome by replacing it with an instrumentation amplifier. Like a difference amplifier, instrumentation amplifier measures and/ or amplifies (or attenuates) the difference between two input signals while rejecting the common mode signals completely unlike the op-amp based difference amplifier circuit which passes the common mode at the input to the output. Also, with op-amps at the front end of the instrumentation amplifier, the input impedance is very high and any impedance mismatch effect is minimized. Commercially available instrumenation amplifier IC's exhibit input impedance in the range of hundreds of mega-ohms and the output impedance in the range of milli-ohms.

Typically, the voltage gain of the instrumentation amplifier is set by single resistor. When the voltage gain is set to unity, the instrumentation amplifier acts as a voltage acquring and measurement circuit.

Therefore, the advantages of using an instrumentation amplifier over op-amp are as follows:

a. Common Mode Rejection Ratio (CMRR) of an in-amp is higher than an opamp and rejects DC, noise, etc. This makes it an excellent choice for acquiring very low voltage signals like ECG, EEG, EMG, EKG, etc. that are sometimes 
buried in noise.

b. Another big advantage, as discussed, of an in-amp over an op-amp is the inamp's ability to reduce impedance mismatch error.

c. Setting the gain of the in-amp is accomplished by a single resistor, and this reduces complexity.

The stages of an instrumenation amplifier and working can be seen in the figure 2.14. The front-end of the instrumentation amplfier are two non-inverting amplifiers and the second stage is a difference amplifier.

The gain an input signal experience in the first stage is $\left(1+\left(2 \cdot R_{5} / R_{G}\right)\right)$. In the second stage, an op - amp is configured as a difference amplifier, where the non-inverting input experiences a gain of $\left(1+R_{4} / R_{3}\right)$ and the inverting input experiences a gain of $-R_{2} / R_{1}$. 

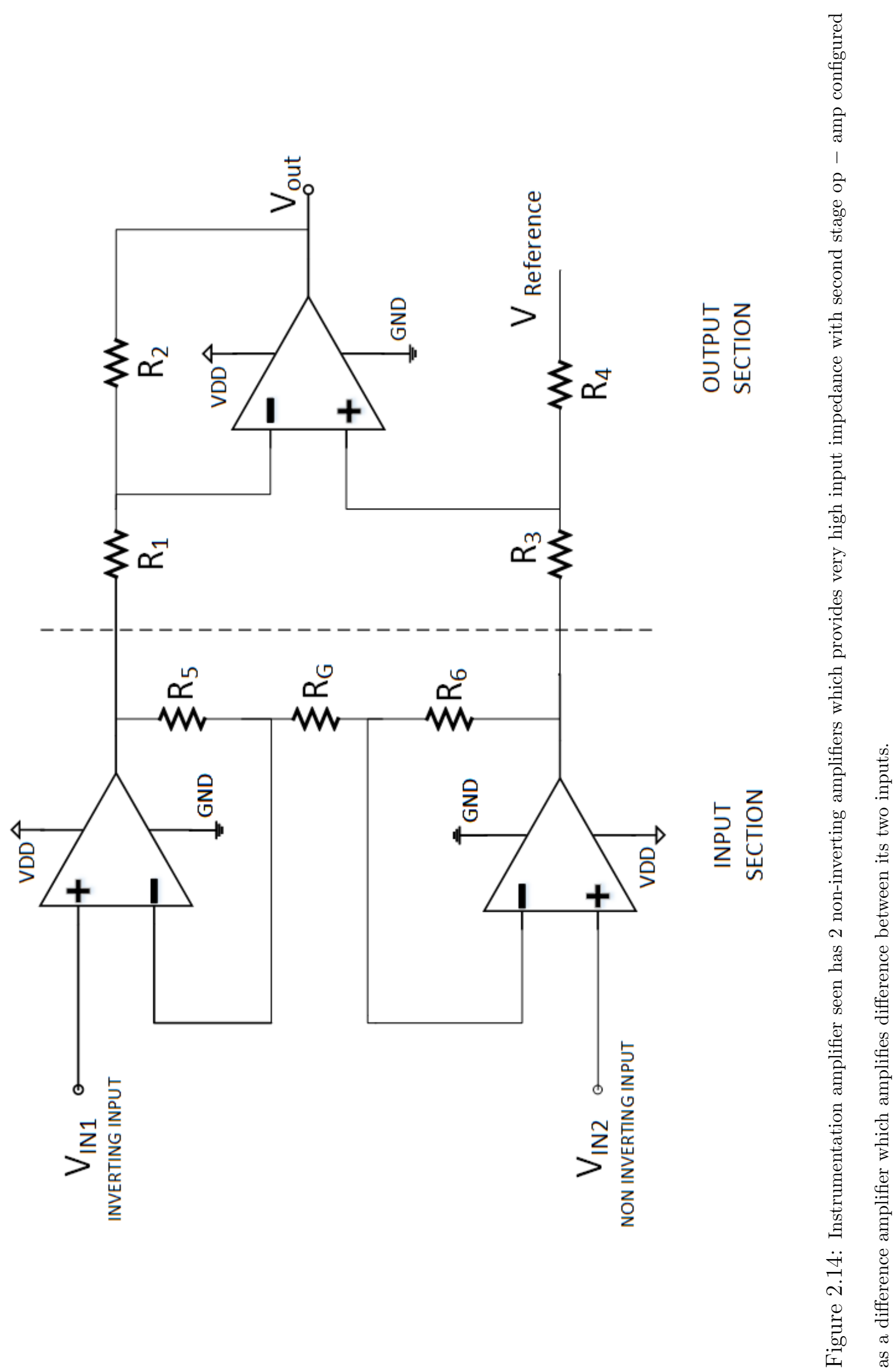
Assuming,

$$
\begin{aligned}
& R_{1}=R_{3} \\
& R_{2}=R_{4} \\
& R_{5}=R_{6}
\end{aligned}
$$

and if the reference voltage is $0 \mathrm{~V}$ and on applying superposition principle, the output voltage signal is

$$
V_{\text {out }}=\left(V_{I N 2}-V_{I N 1}\right) \cdot\left(1+2 \cdot \frac{R_{5}}{R_{G}}\right) \cdot\left(\frac{R_{2}}{R_{1}}\right)
$$

Now, if

$$
R_{1}=R_{2}
$$

and $R_{G}$ is infinte, implies open circuit, the output voltage is

$$
V_{\text {out }}=\left(V_{I N 2}-V_{I N 1}\right)
$$

The instrumentation amplifier works with a gain of 1 and the output is the difference input. 


\subsubsection{Post Signal Processing Circuit}

Signal obtained from the instrumentation amplifier is analog in nature. A user could observe the signal on an oscilloscope and hand calculate the RMS value from the peak value of the signal and when that value is divided by the RMS value of the current injected unknown impedance is found. But, it is not always the case that it is possible to observe the signal on an oscilloscope.

Another method would be is to sample the signal using an ADC and obtain the value digitally. This would mean additional circuitry not to mention embedded coding. Thus a circuit that could directly give the RMS value without any hand calculations or an ADC is a precisely designed rectifier circuit.

Rectifiers are electronic circuits that performs AC to DC. These are of two kinds. a. Half-Wave Rectifier (HWR) - Rectifies only one half of the signal applied to the load.

b. Full-Wave Rectifier (FWR) - Rectifies the complete cycle of the signal applied to the load.

From rectifier theory, for a half-wave rectifier, the ripple voltage is

$$
\text { Ripple Voltage }=\Delta V=\frac{I}{2 . C_{\text {load }} . F r e q}
$$

The average dc value or the mean value is,

$$
\text { Mean } D C \text { Voltage }=V_{\text {peak }}-\frac{I}{2 \cdot C_{\text {load }} \cdot F r e q}
$$

For a full-wave rectifier, the ripple voltage is

$$
\text { Ripple Voltage }=\Delta V=\frac{I}{4 . C_{\text {load }} . \text { Freq }}
$$


The average dc value or the mean value is,

$$
\text { Mean } D C \text { Voltage }=V_{\text {peak }}-\frac{I}{4 \cdot C_{\text {load }} \cdot \text { Freq }}
$$

From the equations it is not difficult to design the circuit with appropriate load capacitor so that the mean voltage is equal to RMS value of a signal.

$$
\begin{aligned}
& V_{R M S}=0.707 . V_{\text {Peak }} \\
& I_{R M S}=0.707 . I_{\text {Peak }}
\end{aligned}
$$

Where, $V_{\text {Peak }}$ and $I_{\text {Peak }}$ are the peak value (or amplitude) of the signal.

\section{Gate Rectifier Circuit}

A diode-less method of rectification is proposed to perform half-wave and full-wave rectification.

Typically, diodes are used in rectifier circuits and the diodes have finite and significant voltage drop across them. For higher voltages this may not be an issue, but for low power circuits the drop is significant. With the proposed method, the drop is zero.

The rectification is done by connecting the inputs to the sources of pass transmission gates and switching them on or off depending on the cycle intended for rectification hence the name gate rectifiers.

\section{Operation of Gate HWR Circuit:}

From the figure $2.15 \mathrm{a}$, if the clock signals to the transmission gate have the same frequency and the transmission gate is 'switched on' during the positive half cycle of the input signal but 'off' during negative half cycle, then only the positive half of the signal is transmitted and this signal can be seen as half-wave rectified form 

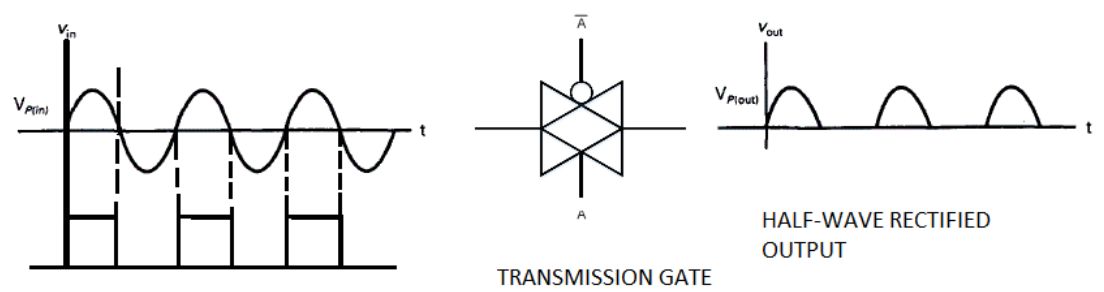

a. GATE HALF-WAVE RECTIFIER

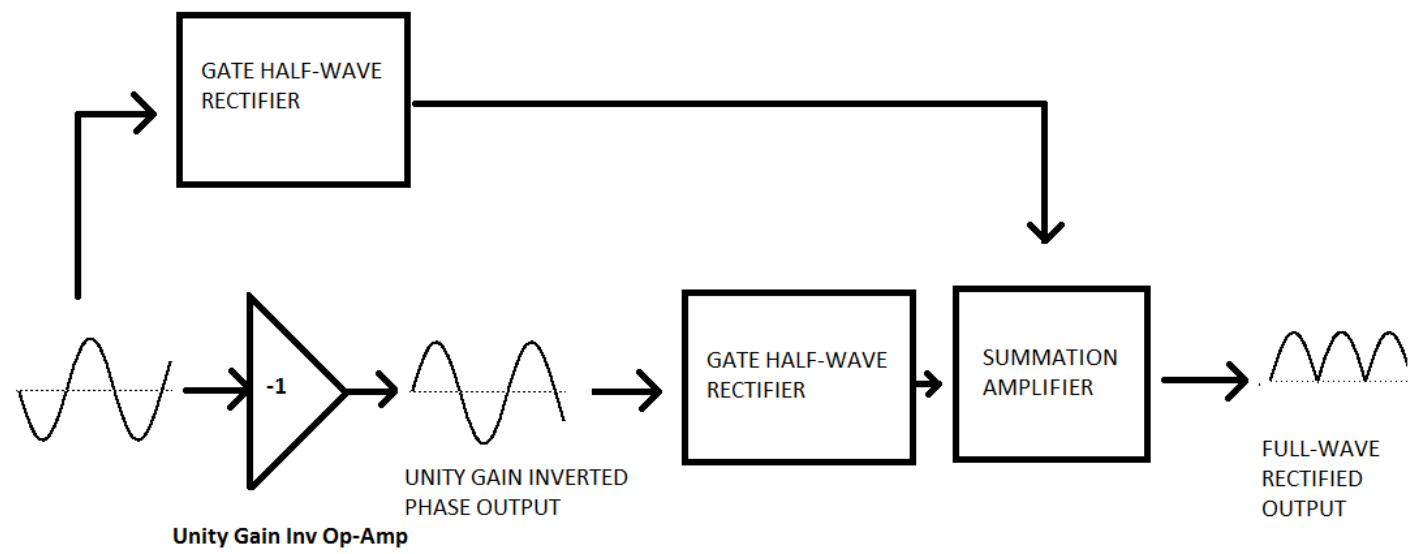

b. GATE FULL-WAVE RECTIFIER

Figure 2.15: In the figure, are block level schematics for gate rectifiers, both, half-wave rectifier (performed by gated half-wave rectifier) and full-wave rectifier (performed by gated full-wave rectifier).

of signal. With a capacitive load connected, one can observe a rectified DC voltage signal with ripples. 

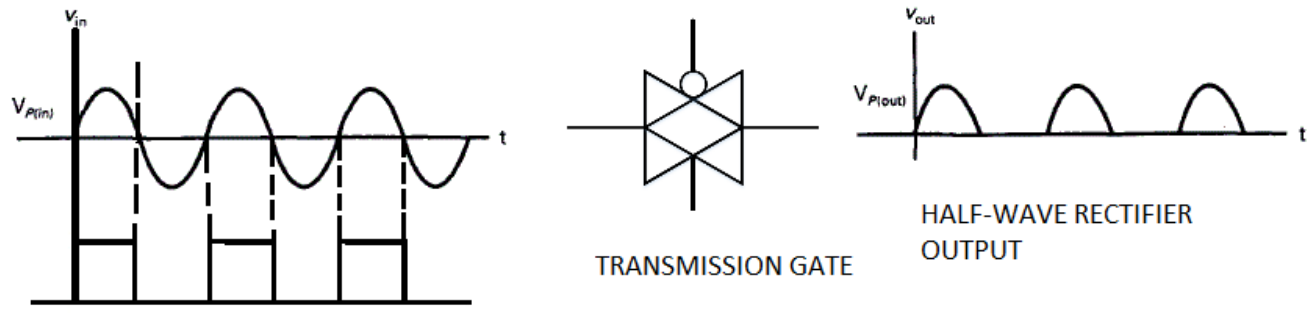

a. GATE HALF-WAVE RECTIFIER

Figure 2.16: In the figure, input signal is given to switching transmission gate which acts as a switch and produces rectified output. 


\section{Operation of Gate FWR Circuit:}

In the figure $2.15 \mathrm{~b}$, when an input signal is applied to a unity gain inverter and the output is obtained, phase inversion is observed. Now, performing half-wave rectification on this signal and applying the output signal as an input to a summation amplifier along with the half-wave rectified output of the original signal, the output of summation amplifier is observed to a full wave rectified output. Now, with a load capacitor one can observe a rectified DC voltage signal with ripples.

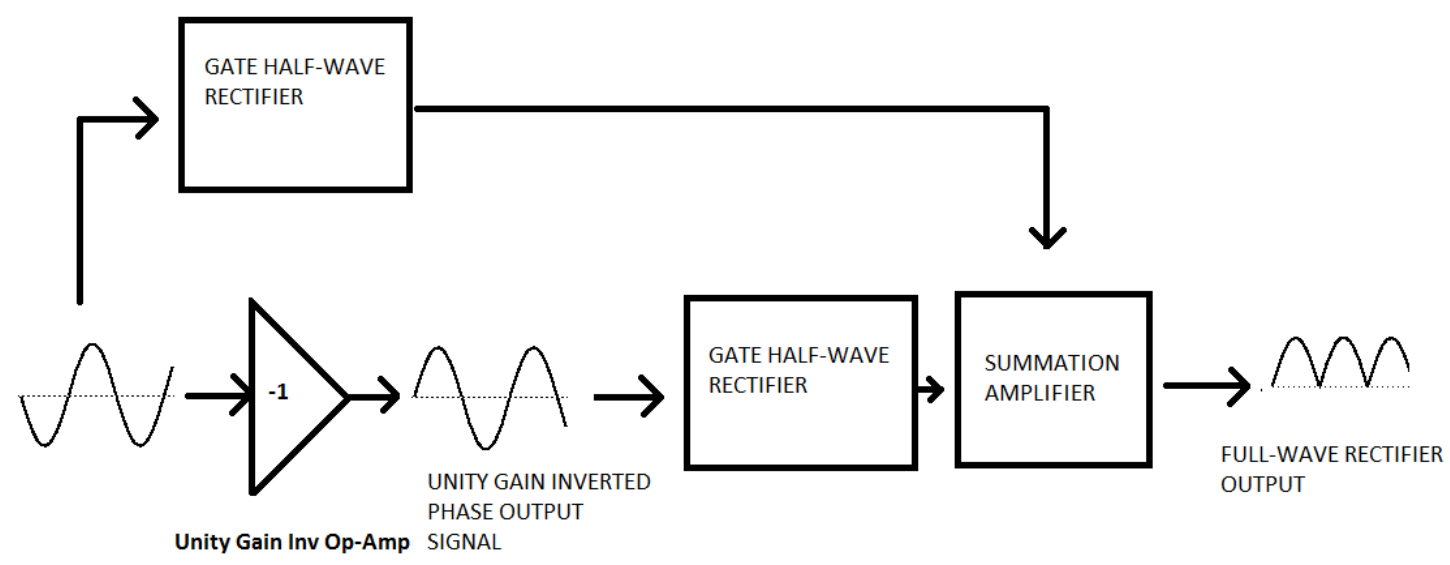

b. GATE FULL-WAVE RECTIFIER

Figure 2.17: In the figure, fully rectified output is obtained from summation of two half-wave rectified signals.

\subsection{Literature Review of Bioimpedance Circuits}

Typically, bioimpedance is determined using a system-level circuit designed with different components available from various vendors in the market such as Texas Instruments, Analog Devices, etc. Op-amps from before mentioned vendors can be 
purchased along with other necessary electrical components and circuits like current sources and instrumentation amplifiers can be designed and developed. This approach is not always cost-effective, not to mention other design considerations like IC's with compatible power supplies, board space, matching resistors, etc. Taking these reasons along with rising interest in applications of bioimpedance into consideration, it is understandable that there is a need for an integrated circuit solution to the problem of determination of bioimpedance.

There are very few IC's in the market that are dedicated to finding bioimpedance. Two IC's that are available in the market and that determine bioimpedance are:

a. AFE4300 - Texas Instruments

b. HM301D - STMicroelectronics

\subsubsection{AFE4300}

The integrated circuit AFE4300 [7] is the Texas Instruments Inc. analog frontend solution which integrates weigh scale measurement circuit and bioimpedance measurement circuit. It is a $12-\mathrm{mm} \times 12-\mathrm{mm}$ TQFP-80 packaged chip which is a priced at 2.50 dollars with a minimum order of 1000 chips in quantity.

The AFE4300 determines bioimpedance by empolying tetra-polar bioimpedance measurement technique. Additionally, the chip has three Body Composition Measurement $(\mathrm{BCM})$ channels enabling segmental bioimpedance measurements which can increase the overall accuracy of the bioimpedance measured.

\section{Architecture}

Body composition measurements using AFE4300 are obtained by injecting known current and measuring the voltage response of the subject. The voltage response 
acquired is processed either by $\mathrm{AC}$ rectification method or $\mathrm{I} / \mathrm{Q}$ demodulation method.

A current of RMS magnitude $375 \mu A$ is injected into the Subject Under Test (SUT) with an op-amp based voltage-to-current source. An AC voltage sine-wave input signal to the current source is produced by a 6-bit, 1-MSPS Digital-to-Analog Converter (DAC).

The voltage response produced by the SUT is measured by a differential amplifier. The measured voltage is then rectified and its amplitude is extracted and measured by a 16-bit Analog-to-Digital Converter (ADC).

\section{Limitations of AFE4300}

The limitations of AFE4300 in measuring bioimpedance are:

a. In the voltage-to-current converter current source seen in the figure, a $1.5 \mathrm{~K} \Omega$ resistor in series voltage input signal from the DAC and connected to the input termial of the op-amp reduces the input impedance of the current source.

b. AFE4300 supports multi-frequency operation but the current injected into the SUT cannot be controlled by the designer and it is equal to $375 \mu \mathrm{A}$ (the value may change depending on the tolerance of the $1.5 \mathrm{~K} \Omega$ resistor used in the current source).

c. The differential amplifier seen in the figure to measure the voltage response is dependent on the value of the resistors, their tolerance and their matching. Lower resistor values result in reduced input impedance and, in any case, the input impedance of the differential amplifier is lower than the input impedance of an instrumentation amplifier which has op-amp based buffers at the input front-end of the circuit. Reduced input impedance at the input front-end of the voltage 
measuring circuit makes the circuit vulnerable to impedance mismatch resulting in reduced CMMR.

\subsubsection{HM301D}

HM301D [8] is an integrated circuit from STMicroelectronics which is a highly integrated circuit biopotential signal acquisition system, like ECG, EEG and EMG, which can also measure bioimpedance. It is a 6-mmx 6-mm chip with a 40-lead Large Grid Array (LGA) package.

\section{Architecture}

It is apparent from the datasheet for HM301D the chip supports bi-polar and tetra-polar bioimpedance technique to determine bioimpedance.

The current source circuit is capable of injecting a current of value $5 \mu \mathrm{A}, 10 \mu \mathrm{A}$ or $20 \mu \mathrm{A}$ that is chosen by the designer. The frequency of injection seen in the datasheet is $31.25 \mathrm{kHz}$.

The voltage measurement circuit utilized by the IC is an instrumentation amplifier with gains of values 8 and 16 only.

Post processing of the output signal from the instrumentation amplifier is performed by a sigma-delta ADC and fed to the IQ-demodulator. The IQ-demodulator splits the real and imaginary components and provides the bioimpedance value.

\section{Limitations of HM301D}

The drawbacks of the integrated circuit HM301D are:

a. It's limitation in magnitude of the injected current. The maximum amount of current that can be injected is still much lower than the typically injected values. The magnitude of current is so low that it necessitates a very high signal-to-noise 
ratio (SNR) and high common mode rejection ratio for the voltage measuring circuit resulting in a complicated circuit which would require very high number of precisely matched transistors.

b. The supply voltages and the current consumption is high implying a high power consumption.

\subsection{Chapter Summary}

In this Chapter, the background on determining bioimpedance is detailed. Relevant techniques have been introduced along with the high-level electrical circuits that implement them. The advantages of tetra-polar technique and the disadvantages of bi-polar technique have been discussed.

Potentiostatic excitation and galvanostatic excitation methods to determine bioimpedance have been discussed. The proposed galvanostatic excitation based architecture for the circuit is shown. The proposed architecture comprises of current source circuits, voltage measuring circuits and post-processing circuits. International standards impose a restriction on the amount of current that can be injected to determine bioimpedance based on the frequency of operation. The standard for the current and the concept of bioimpedance forms the basis for specifications of the system and its sub-circuits. The rationale behind the requirements and electrical characteristics of the sub-circuits of the system have been discussed at length. The specifications for the current source, voltage measurement circuit and the operational amplifier haven been established.

Different types of current sources, voltage measuring circuits, and post-processing circuits have been explored. Pros and cons of the different circuits have been 
analyzed. Based on the requirements, mirrored modified Howland current, instrumentation amplifier and a diode-less rectifier circuit have been used to build the bioimpedance system.

The designed circuit has been compared with commercially available IC's like AFE4300 and HM301D. From the datasheets for AFE4300 [7] and HM301D, a few imporatant specifications relevant to bioimpedance are compared in the comparison table 2.4 shown below.

Also, the table 2.5 outlines comparison between operational amplifiers published and the operational amplifier designed during the course of this thesis. 


\begin{tabular}{|c|c|c|c|}
\hline Parameter & AFE4300 & HM301D & This work \\
\hline VDD & $2 \mathrm{~V}$ & $1.62 \mathrm{~V}$ & $1.5 \mathrm{~V}$ \\
\hline VSS & $0 \mathrm{~V}$ & $0 \mathrm{~V}$ & $0 \mathrm{~V}$ \\
\hline CMRR & $95 \mathrm{~dB}$ & $100 \mathrm{~dB}$ & $\sim 135 \mathrm{~dB}$ \\
\hline Offset & $80 \mu \mathrm{V}$ & - & $34 \mu \mathrm{V}$ \\
\hline Power consumption & $1.94 \mathrm{~mW}$ & $3.56 \mathrm{~mW}$ & $818.2 \mu \mathrm{W}$ \\
\hline Injection Current & $375 \mu \mathrm{A} \mathrm{RMS}$ & $5 \mu \mathrm{A}, 10 \mu \mathrm{A}, 20 \mu \mathrm{A}$ & Upto $700 \mu \mathrm{A}$ \\
\hline Injection Frequency & only $50 \mathrm{kHz}$ & only $31.25 \mathrm{kHz}$ & Upto $100 \mathrm{kHz}$ \\
\hline
\end{tabular}

Table 2.4: Comparison of Specifications of the IC with AFE4300 and HM301D chips.

The injected current is a function of input voltage. For a gain setting resistor of $1 K \Omega$ belonging to the current source, to inject a current of $700 \mu \mathrm{A}$, an input of $700 \mathrm{mV}$ would be applied, which is a large signal. Therefore, by reducing the value of the resistor it is possible to produce high output currents for low voltage input signals. The power consumption specification of the chip designed is at DC conditions. 


\begin{tabular}{|c|c|c|c|c|c|c|c|c|}
\hline 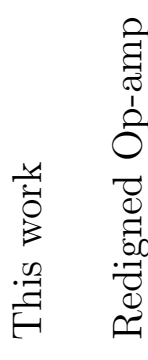 & 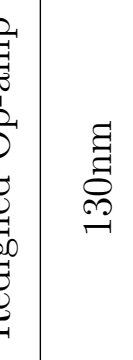 & مُ & 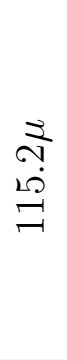 & 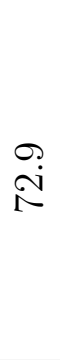 & 공 & $\stackrel{?}{\stackrel{?}{*}}$ & 1 & 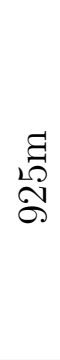 \\
\hline 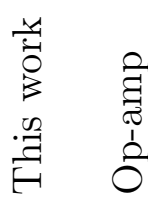 & 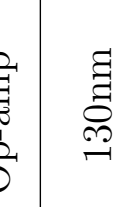 & نجّ & 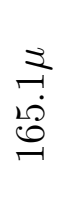 & $\begin{array}{r}\stackrel{1}{\infty} \\
\stackrel{\infty}{0}\end{array}$ & 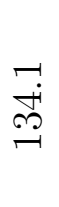 & $\stackrel{-}{\frac{1}{N}}$ & $\sum_{\substack{\sum_{j} \\
\dot{q}}}^{\infty}$ & 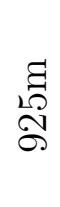 \\
\hline 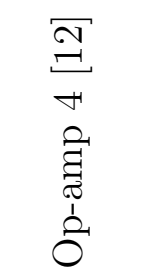 & 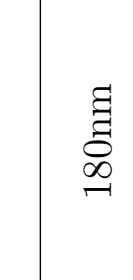 & $\neg$ & $\begin{array}{l}3 \\
\text { 咅 } \\
120\end{array}$ & 온 & $\begin{array}{l}\infty \\
\infty \\
\infty \\
1\end{array}$ & 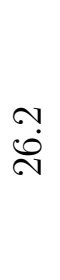 & $\frac{\varangle}{z}$ & $\frac{\varangle}{z}$ \\
\hline 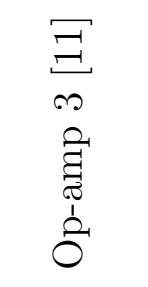 & 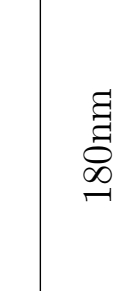 & - & 胥 & $\underset{\forall}{+H}$ & $\underset{\infty}{H}$ & $\begin{array}{l}\sqrt{20} \\
\stackrel{10}{\circ}\end{array}$ & $\frac{\varangle}{z}$ & $\frac{\varangle}{z}$ \\
\hline 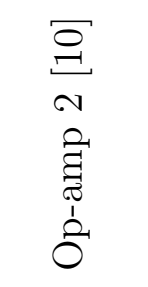 & 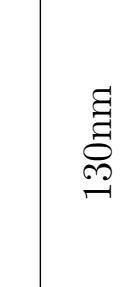 & $\ddot{\leftrightarrow}$ & 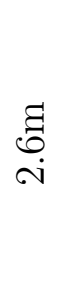 & 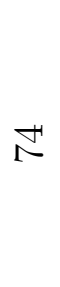 & $\stackrel{\text { }}{\sim}$ & $\stackrel{\wp}{\varrho}$ & $\underset{\sim}{\mathbb{N}}$ & $\stackrel{\sim}{\sim}$ \\
\hline $\begin{array}{l}\bar{a} \\
\vec{\sigma} \\
a \\
a \\
\tilde{T} \\
\vdots \\
0\end{array}$ & 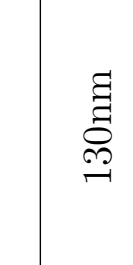 & $\stackrel{\sim}{\sim}$ & $\underset{\Xi}{\stackrel{\Xi}{\sigma}}$ & 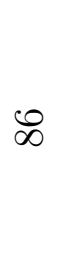 & $\sigma$ & 120 & $\nexists$ & 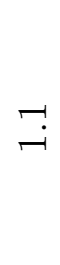 \\
\hline 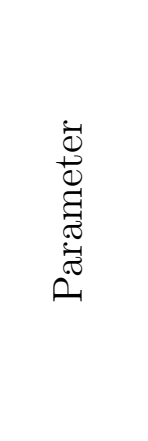 & 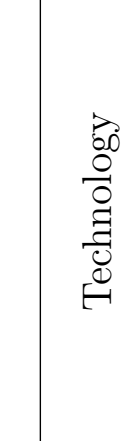 & 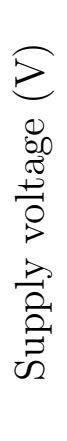 & 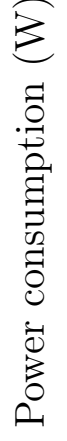 & 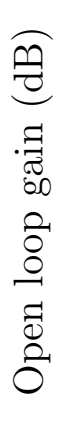 & 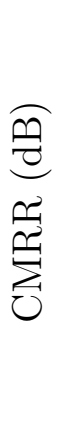 & 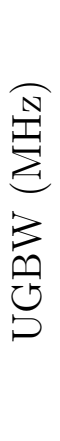 & 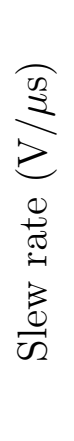 & 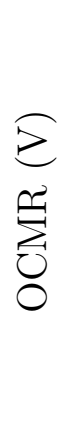 \\
\hline
\end{tabular}




\section{Chapter 3}

\section{Design and Simulations}

This Chapter presents the design and implementation of the bioimpedance system and its sub-circuits. The description and operation of the designed operational amplifier, current source circuit, instrumentation amplifier and the post-processing circuits are presented along with the simulation results of various analyses. For the purposes of simulations, a single-supply system is chosen with a power supply of $1.5 \mathrm{~V}$ with $0 \mathrm{~V}$ ground reference unless specified otherwise. Also, most transient simulations are performed at a frequency of $10 \mathrm{kHz}$.

The ciruits are designed and implemented using IBM 130nm technology. Cadence virtuoso is used for developing schematics and layout editor for layouts. The simulator used for simulations is Spectre.

\subsection{Operational Amplifier}

A high gain, high CMRR and low power operation amplifier, aka op-amp, is designed to meet the specifications that are presented in the table 2.1. 


\subsubsection{Circuit Description}

The operational amplifier with a constant $G_{m}$-bias circuit figures the following stages:

a. Differential input gain stage I

b. Differential Input gain stage II

c. Common source amplifier stage (CS stage)

d. Buffer stage

Two differential gain stages are chosen for the op-amp to increase the gain of the op-amp and improve its ability to reject common mode signal. The CS stage provides additional gain to the signal. A buffer stage is added to the circuit to drive resistive loads, as bioimpedance is highly resistive in nature.

The 4 stages of the op-amp stated in the previous section, 3.1.1, are seen in the op-amp shown in the figure 3.1.

The resistor in the constant gm-bias stage sets the current in the bias circuit and decides the bias current in the rest of the circuit. The gate-source voltage of T16 and the drop in voltage across the bias resistor is equal to the gate-source voltage of the T14 transistor. The width of T16 is designed to sink the same amount of current as that of T14 for the gate-source voltage, $V_{g s 16}$, equal to $\left(V_{\text {gs } 14}-R_{\text {bais }} \cdot I_{\text {bias }}\right)$. The $\mathrm{T} 14$ transistor drives the current in the T8 and T10 transistors. The transistor pairs T10 and T11, T9 and T8 are current mirrors and because of similar widths and lengths the current driven in T9 and T11 are the same as $\mathrm{T} 8$ and T10. This setup ensures that the currents in both the branches are the same and transconductance of the transistors is constant.

The first and the second differential pair gain stages are biased by the P-channel 
MOSFETs T20 and T13 respectively. Belonging to the first differential pair stage, T0 and T1 are the P-channel differential pair MOSFET transistors with T18 and T19 N-channel MOSFETs acting as current mirror loads. In the second differential pair stage, T7 and T12 are the P-channel differential pair MOSFET transistors with T2 and T4 acting as their current mirror loads. It is implicit that the differential pair transistors match each other and so do the current mirror load pairs.

The T6 N-channel transistor acts as the common source amplifier in the CS stage with the T5 PMOS transistor as its load. The capacitor and an NMOS transistor, T3, seen in the CS stage provide miller compensation to the circuit.

The NMOS transistors T22 and T21 form the buffer stage of the circuit with T21 acting as the load and T22 acting the common drain transistor.

The subc component in the figure 3.1 is used to model the substrate.

The table 3.1 displays the value of parameters of the components in the schematic. 


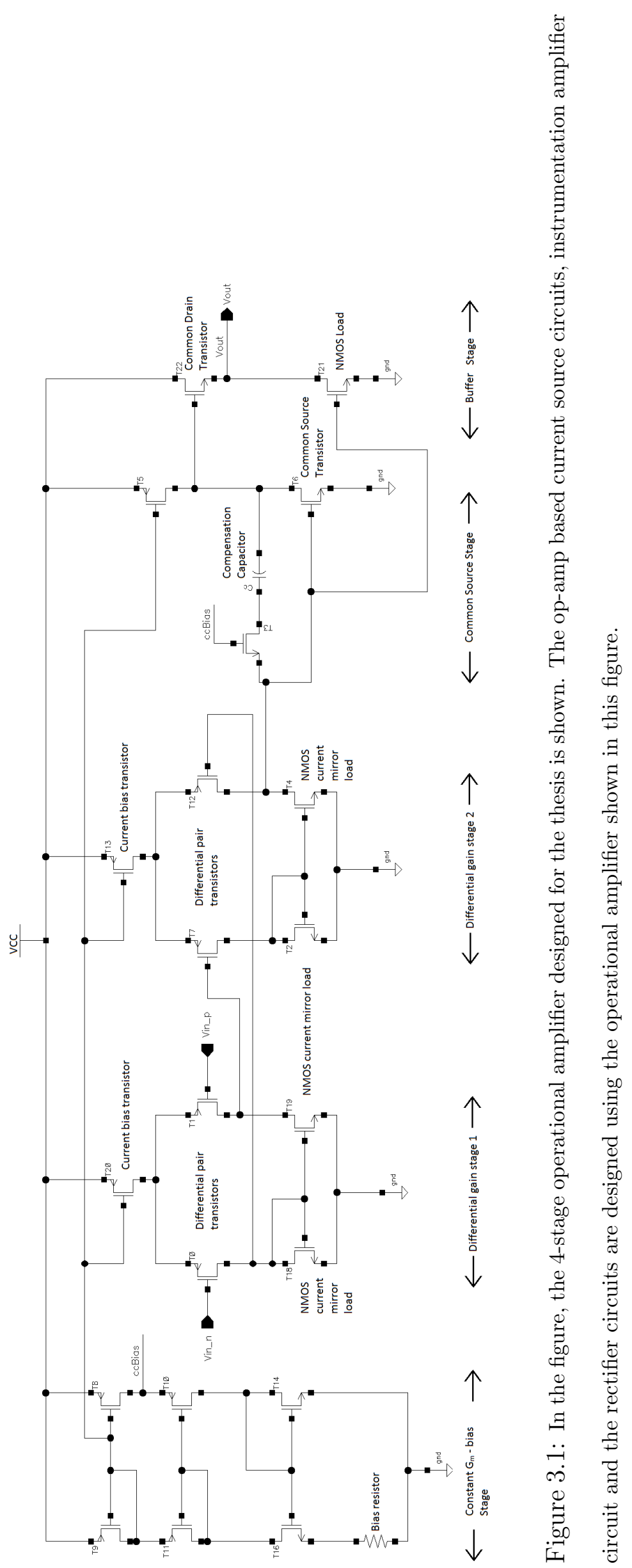




\begin{tabular}{|c|c|c|}
\hline Component & Type & Value \\
\hline T0 & PMOS & $\mathrm{W}=10 \mu \mathrm{m} ; \mathrm{L}=480 \mathrm{~nm}$ \\
\hline $\mathrm{T} 1$ & PMOS & $\mathrm{W}=10 \mu \mathrm{m} ; \mathrm{L}=480 \mathrm{~nm}$ \\
\hline $\mathrm{T} 7$ & PMOS & $\mathrm{W}=10 \mu \mathrm{m} ; \mathrm{L}=480 \mathrm{~nm}$ \\
\hline $\mathrm{T} 12$ & PMOS & $\mathrm{W}=10 \mu \mathrm{m} ; \mathrm{L}=480 \mathrm{~nm}$ \\
\hline $\mathrm{T} 2$ & NMOS & $\mathrm{W}=960 \mathrm{~nm} ; \mathrm{L}=360 \mathrm{~nm}$ \\
\hline $\mathrm{T} 4$ & NMOS & $\mathrm{W}=960 \mathrm{~nm} ; \mathrm{L}=360 \mathrm{~nm}$ \\
\hline $\mathrm{T} 18$ & NMOS & $\mathrm{W}=960 \mathrm{~nm} ; \mathrm{L}=360 \mathrm{~nm}$ \\
\hline $\mathrm{T} 19$ & NMOS & $\mathrm{W}=960 \mathrm{~nm} ; \mathrm{L}=360 \mathrm{~nm}$ \\
\hline T6 & NMOS & $\mathrm{W}=5 \mu \mathrm{m} ; \mathrm{L}=480 \mathrm{~nm}$ \\
\hline $\mathrm{T} 5$ & PMOS & $\mathrm{W}=20 \mu \mathrm{m} ; \mathrm{L}=360 \mathrm{~nm}$ \\
\hline $\mathrm{T} 20$ & PMOS & $\mathrm{W}=5 \mu \mathrm{m} ; \mathrm{L}=360 \mathrm{~nm}$ \\
\hline $\mathrm{T} 13$ & PMOS & $\mathrm{W}=4.8 \mathrm{u} \mu \mathrm{m} ; \mathrm{L}=360 \mathrm{~nm}$ \\
\hline $\mathrm{T} 22$ & NMOS & $\mathrm{W}=6 \mu \mathrm{m} ; \mathrm{L}=360 \mathrm{~nm}$ \\
\hline $\mathrm{T} 21$ & NMOS & $\mathrm{W}=6 \mu \mathrm{m} ; \mathrm{L}=360 \mathrm{~nm}$ \\
\hline $\mathrm{T} 3$ & NMOS & $\mathrm{W}=360 \mathrm{~nm} ; \mathrm{L}=3.6 \mu \mathrm{m}$ \\
\hline $\mathrm{T} 14$ & NMOS & $\mathrm{W}=720 \mathrm{~nm} ; \mathrm{L}=360 \mathrm{~nm}$ \\
\hline $\mathrm{T} 16$ & NMOS & $\mathrm{W}=2 \mu \mathrm{m} ; \mathrm{L}=360 \mathrm{~nm}$ \\
\hline $\mathrm{T} 8$ & PMOS & $\mathrm{W}=2.68 \mu \mathrm{m} ; \mathrm{L}=360 \mathrm{~nm}$ \\
\hline T9 & PMOS & $\mathrm{W}=2.68 \mu \mathrm{m} ; \mathrm{L}=360 \mathrm{~nm}$ \\
\hline $\mathrm{T} 11$ & PMOS & $\mathrm{W}=360 \mathrm{~nm} ; \mathrm{L}=360 \mathrm{~nm}$ \\
\hline $\mathrm{T} 10$ & PMOS & $\mathrm{W}=360 \mathrm{~nm} ; \mathrm{L}=360 \mathrm{~nm}$ \\
\hline Bias resistor & - & $12.5 \mathrm{~K} \Omega$ \\
\hline$C_{c}$ & - & $254 \mathrm{fF}$ \\
\hline
\end{tabular}

Table 3.1: Designed Operational amplifier components and their values 


\subsubsection{Circuit Operation}

This section deals with working of the op-amp focusing on the differential gain stages of the op-amp and the topology. Starting with the understanding of the opamp's single differential gain stage by investigating the source of increased gain, simulations of both differential pairs with current mirror loads and normal active loads are presented to testify the contrast.

\section{Differential gain stage with transistor active loads}

The differential gain stage of a typical op-amp is as seen in the figure 3.2.

In the figure 3.2, M4 acts as a current source. M2 and M3 are differential pair

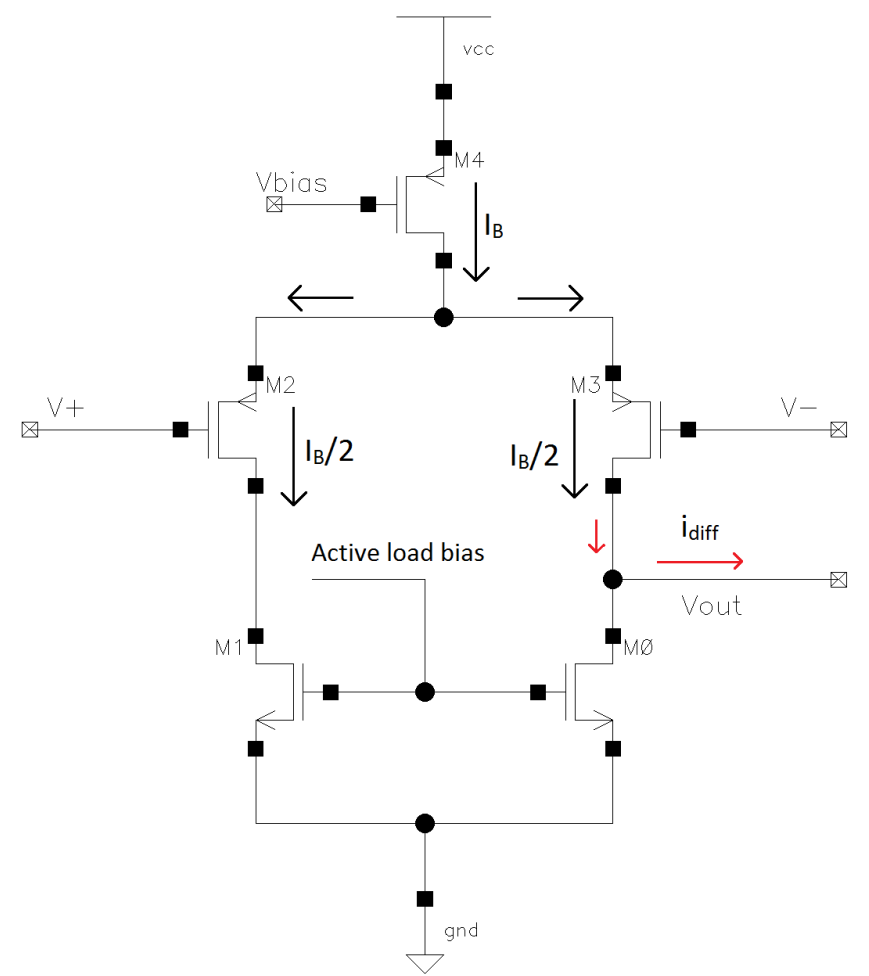

Figure 3.2: A typical differential gain stage of an op-amp with PMOS differential pair transistors with NMOS transistors acting as active loads.

transistors that amplify the voltage input signal applied at their gates. M1 and M0 are active loads that are biased by applying a DC input. In the absence of a small 
AC voltage signal, the currents, $I_{B} / 2$ in the two branches of the differential gain stage are equal and are equal to half the bias current, $I_{B}$. When out-phase AC signals are applied at the inputs, $\mathrm{V}+$ and $\mathrm{V}-$, the current increases in one branch and decreases in the other equally with a gain factor called transconductance $\left(g_{m}\right)$. The small signal AC current, shown in the figure 3.2 as $i_{\text {diff }}$, that is produced as a result of the application of the input flows into the load connected to the output of the op-amp.

The small signal circuit of the typical differential gain stage presented in the figure 3.2 is seen in the figure 3.3 .

In the figure 3.3, a small signal input, $V_{i n_{p}}$ applied at the gate of M2 results in

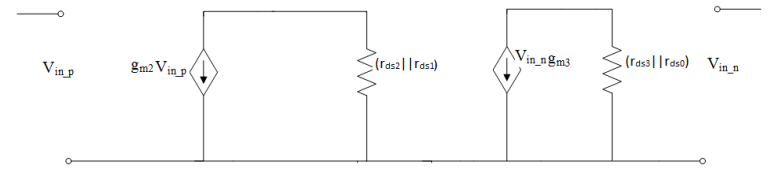

Figure 3.3: The small signal model of a typical differential gain stage of an op-amp

a current of magnitude $g_{m 2} V_{i n_{p}}$ flowing into the output impedance of the M2 and M1 transistors, $r_{d s 2} \| r_{d s 1}$. Similarly, a small signal input, $V_{i n_{n}}$, which is anti-phase with signal applied at the gate of M2, results in a current of magnitude $g_{m 2} V_{i n_{n}}$ flowing into the output impedance $r_{d s 3}|| r_{d s 0}$.

\section{Differential gain stage with current mirror load}

The differential gain stage of the designed operational amplifier is seen in figure 3.4 . 


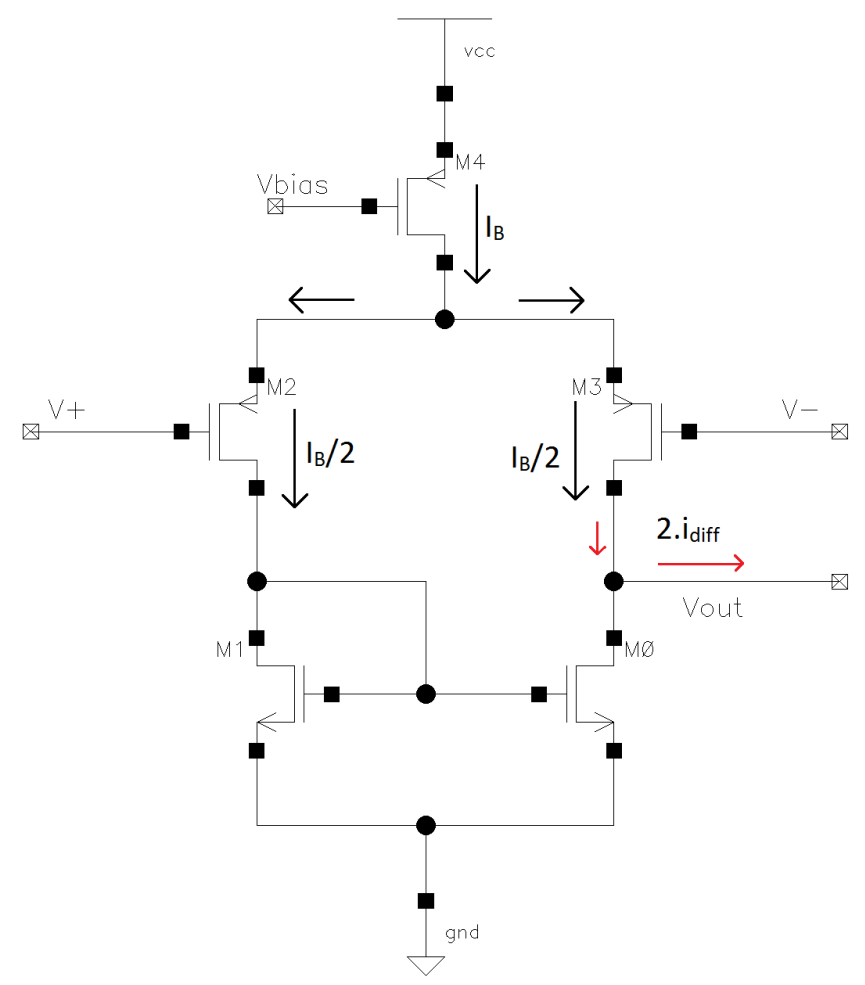

Figure 3.4: A typical differential gain stage of an op-amp with current mirror load 
Contrary to a typical differential gain stage with active loads, the designed opamp has a differential pair with a current mirror load. As seen in the figure 3.4, the DC currents in the two legs of the differential gain stage are equal and equal to half the bias current sourced by M4 transistor which is biased by an external voltage, $V_{\text {bias }}$. When differential small $\mathrm{AC}$ voltage input signals are applied at the gate inputs of M2 and M3, differential small AC current signals are produced with a gain factor equal to the transconductance of the transistors. By the virtue of current mirror load, the differential current generated is doubled, shown in the figure as $2 . i_{\text {diff }}$ because any differential current generated by M1 is 'mirrored' into M0 and the differential current that is doubled in magnitude flows into the output. Thus, the gain of the differential gain stage with current mirror loads is doubled by a diode-connected MOSFET load.

The small signal model of the circuit in the figure 3.4 is shown in figure 3.5. The dependent current sources related to M1 and M0 shown figure 3.5 show that they produce same currents and current of magnitude $g_{m 0} \cdot V_{g s 3}$ (equal to $g_{m 1} \cdot V_{g s 3}$ ) is flowing into the output.

The output impedance of the diode-connected M1 transistor is in parallel with

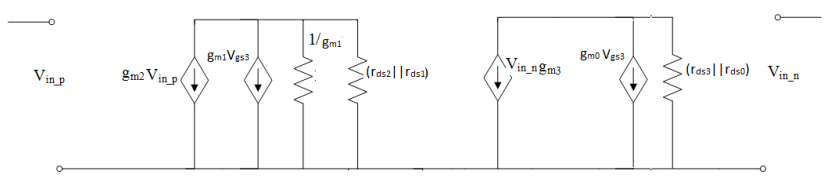

Figure 3.5: The small signal model of a typical differential gain stage of an op-amp with current mirror load

the output impedance of M2. The resulting output impedance is very low. This implies that the differential gain of the M1 and M2 branch of the differential gain 
stage is very low, close to 1 , compared to the M0 and M3 branch of the circuit. Since the gain is very low, a small signal applied at the input of M1 results in an output that is a small signal too as opposed to the output seen at the drain of M3 which has an amplitude higher than the former.

\section{Transient Simulation}

The preceding discussion has elaborated the reason behind the increased gain of the op-amp. The following transient simulation would testify the increased gain. The figures 3.6 and 3.7 show the differential gain stages with active load and current mirror load respectively.

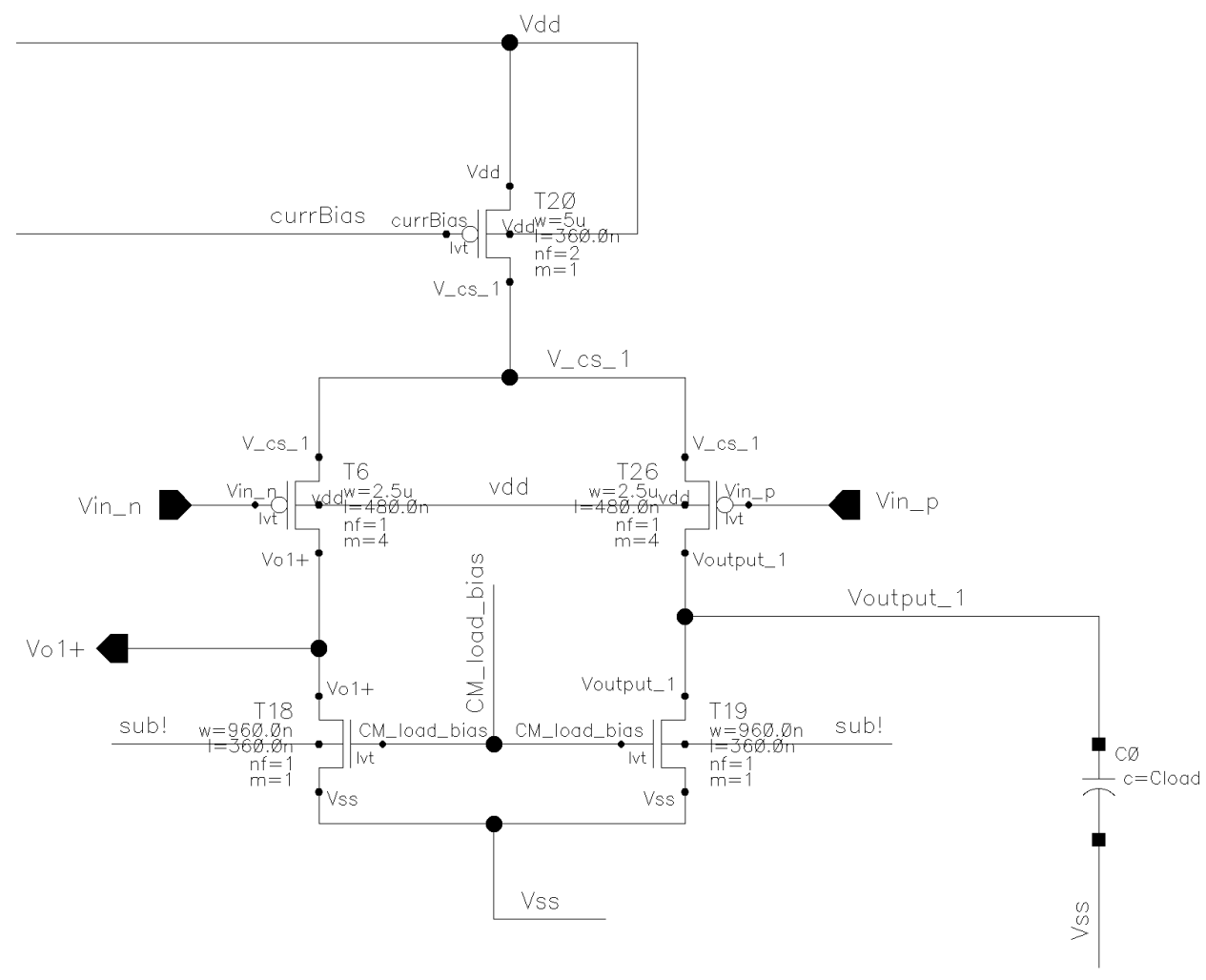

Figure 3.6: Differential pair active load 


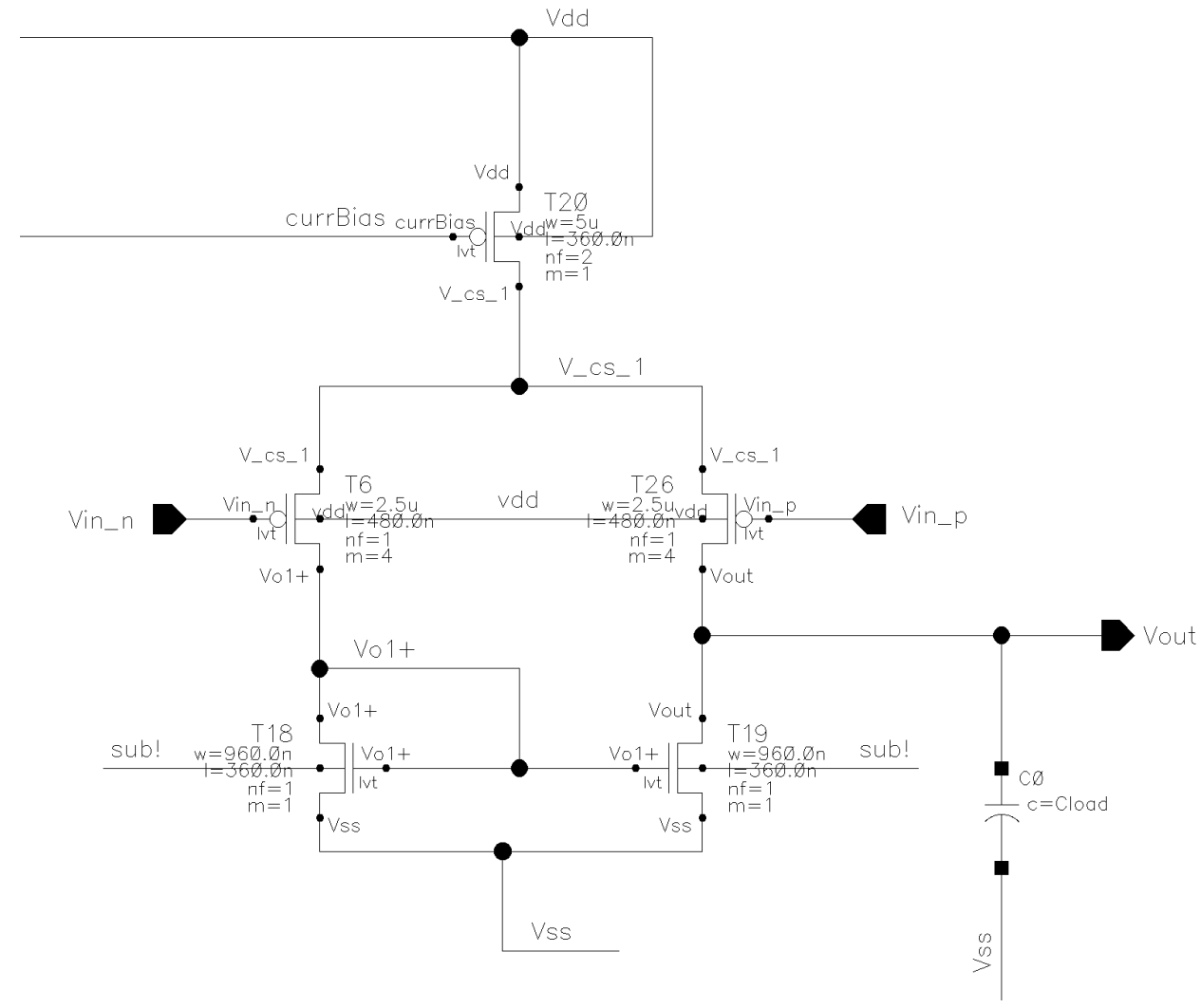

Figure 3.7: Differential pair with current mirror load

To both the differential pair circuits, the inputs applied are differential inputs and the peak to peak amplitude of the input signals is $2 \mu V$. The power supply voltages, Vdd and Vss, are $1.5 \mathrm{~V}$ and $0 \mathrm{~V}$ respectively. The output signals at the drains of the differential pair transistors are plotted along with the inputs. The figures 3.8 and 3.9 demonstrate the transient behavior of the differential gain stages with active loads and current mirror load respectively. 


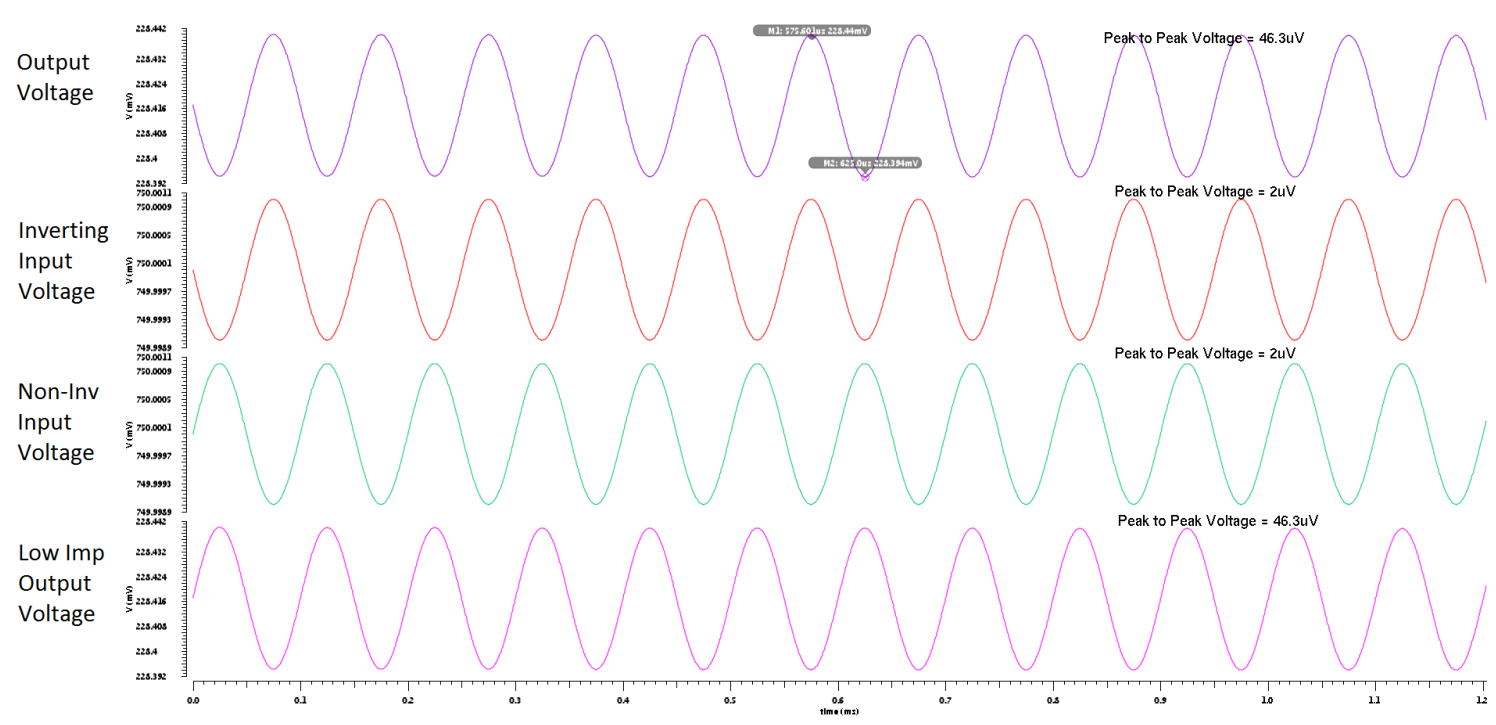

Figure 3.8: From top to bottom: Output voltage signal with a peak to peak voltage of $46.3 \mu \mathrm{V}$, Inverting input voltage signal with a peak to peak of $2 \mu \mathrm{V}$, Non-inverting input voltage signal with a peak to peak of $2 \mu \mathrm{V}$, Low impedance output voltage signal with a peak to peak voltage of of $46.3 \mu \mathrm{V}$ 


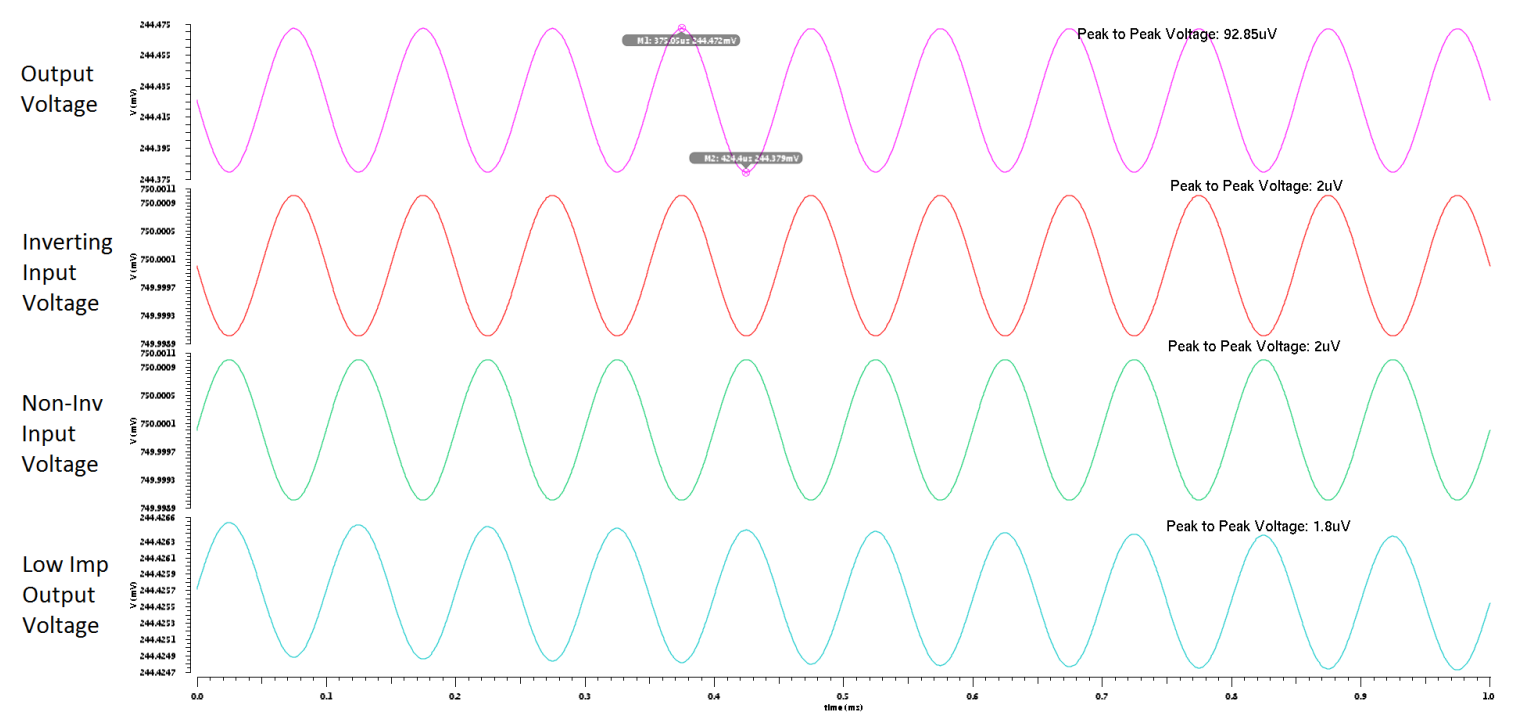

Figure 3.9: From top to bottom: Output voltage signal with a peak to peak voltage of $92.8 \mu \mathrm{V}$, Inverting input voltage signal with a peak to peak of $2 \mu \mathrm{V}$, Non-inverting input voltage signal with a peak to peak of $2 \mu \mathrm{V}$, Low impedance output voltage signal with a peak to peak voltage of of $46.3 \mu \mathrm{V}$. 
The peak to peak output voltage of the differential gain stage with the active load is $46.3 \mu \mathrm{V}$. Whereas, the peak to peak output voltage of the differential gain stage with current mirror load is $92.85 \mu \mathrm{V}$. Hence, it is clear that the gain of the differential gain stage with current mirror load circuit is twice the gain of the circuit with active loads.

As stated before the output voltage observed at the drain of the diode-connected MOSFET is a small signal that is barely amplified by the gain stage and has peak-to-peak voltage of magnitude $1.8 \mu \mathrm{V}$.

The designed op-amp has two cascaded differential gain stages to increase its overall gain. In case of cascaded gain stages with active load, the active loads of the gain stages do not disturb the source node of the differential pair transistors. However, this is not the case with the current mirror load transistors.

If the high impedance output from the first stage is fed as an input to a differential pair P-channel MOSFET with the diode-connected NMOS transistor load as shown in the figure 3.10 the node to which the sources of the differential pair transistors are connected to is no longer an AC ground but a small signal of amplitude comparable to the input. The reason for this is that the diode-connected MOSFET drives the drain of the current bias transistor to oscillate because of the available drain-source voltage margin on the current bias transistor, T13, in which the transistor is still in saturation sourcing the bias current that the differential pair transistors need and to keep itself in saturation. The phase of the small signal at the drain of the current bias transistor is out of phase with the input at the gate of the T26 transistor (P-channel MOSFET at the high impedance output). This leads to an increased gate-to-source voltage at the T26 transistor. The increase 
gate to source voltage increases the flow of small signal current into the output. At the output, along with the increased current due to the increased input gatesource voltage is the increased current due to current mirror action between the T4 and T5. The increased currents account for the small magnitude input at the second stage, thus preserving the gain of the stage.

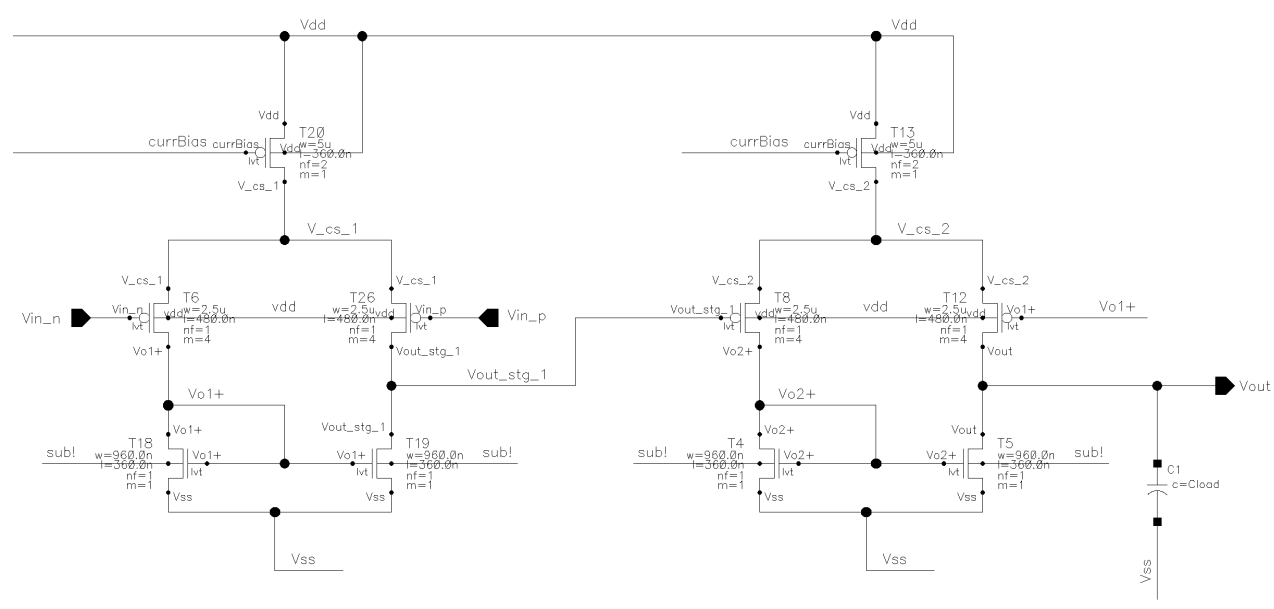

Figure 3.10: Cascaded differential gain stages 
The following figure 3.11 of plots with input, output and intermediate signals of the op-amp demonstrates the transient behavior of the circuit shown in figure 3.10. The examination of amplitudes and peak to peak voltages justify the above theory regarding the operation of the op-amp.

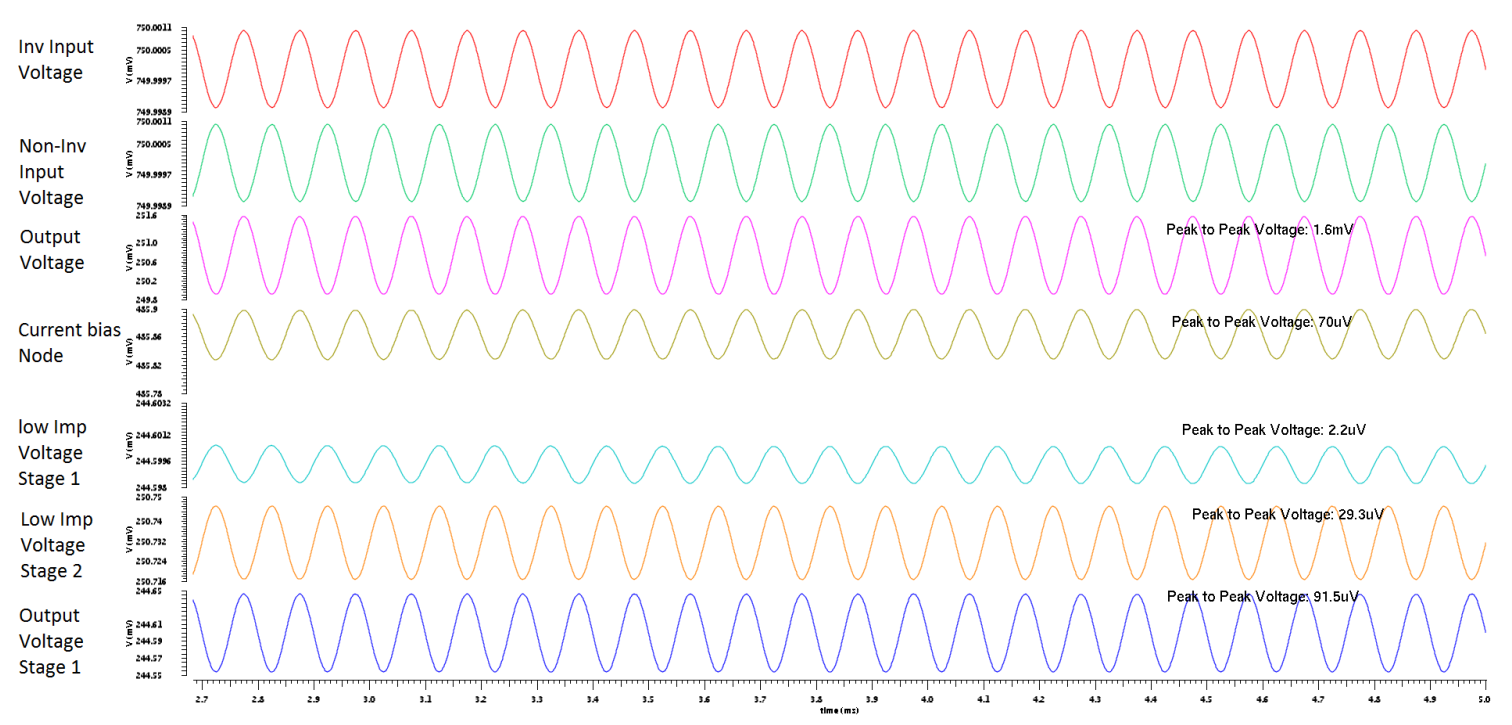

Figure 3.11: Output voltage signal with a peak to peak voltage of $92.8 \mu \mathrm{V}$, Inverting input voltage signal with a peak to peak of $2 \mu \mathrm{V}$, Non-inverting input voltage signal with a peak to peak of $2 \mu \mathrm{V}$, Low impedance output voltage signal with a peak to peak voltage of of $1.8 \mu \mathrm{V}$, Output voltage signal with a peak to peak voltage of $1.6 \mathrm{muV}$, Voltage signal at the current bias transistor drain of the second stage, Low impedance output of the first stage, Low impedance output of the second stage, High impedance output of the first stage with a peak to peak voltage of $91.5 \mu \mathrm{V}$.

The drain of the current source transistor in the second stage (the node is named ${ }^{\prime} V \_c s \_2$ ' in the circuit shown in the picture 3.10 ) exhibits small signal behavior with an amplitude comparable to the high impedance input that the branch is applied with. As mentioned before, this is mainly because the diode-connected MOSFET load is always in saturation and in an attempt to drive the bias current 
that it is designed to the MOSFET load drives the ' $V_{-} c s_{-} 2^{\prime}$ node to oscillate with a small amplitude within the drain-source voltage margin of the current bias transistor. The small signal ensures that the DC bias current is not disturbed and the transistor is not driven out of saturation. Although the drain of the T4 transistor oscillates with a comparable amplitude, compared to the input, the amplitude of the small signal is not large enough to cause large changes in the current driven by the transistor.

The case with high impedance output from the first stage connected to the diodeconnected MOSFET load branch of the second stage is examined, but it'd be incomplete without examining the case of low impedance output feeding the same branch. The circuit is shown in the figure 3.12.

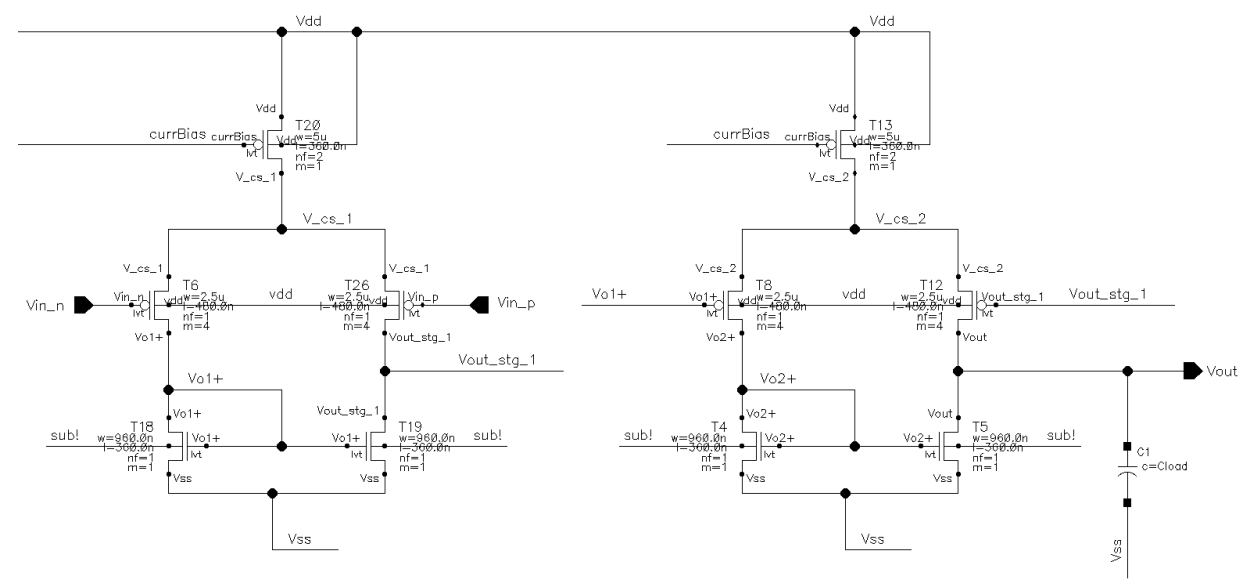

Figure 3.12: In the figure, notice that the high impedance output at the node Vout_stg_1 is connected to T12 transistor branch with high output impedance active load transistor T5, and the low impedance output at the node $V o 1+$ is connected to the $\mathrm{T} 8$ transistor branch with low output impedance diode-connected MOSFET T4.

The transient behavior of the circuit seen in figure 3.12 is shown in the figure 3.13. 
Since the amplitude of the low impedance input is low and the output impedance of the branch that it is applied to is low, the output signal is hardly amplified input. Consequentially, the oscillation of the $V_{-} c s_{-} 1$ is a small signal with very low amplitude and so is the case with the drain output of the T8 transistor. However, any small signal variation causes small signal current and the T4 transistor mirrors the current in the other other branch of the stage preserving the overall differential output current and keeps the gain high. As the overall differential current output is doubled compared to the case with active loads, the gain of the differential gain stage with current mirrors is also doubled.

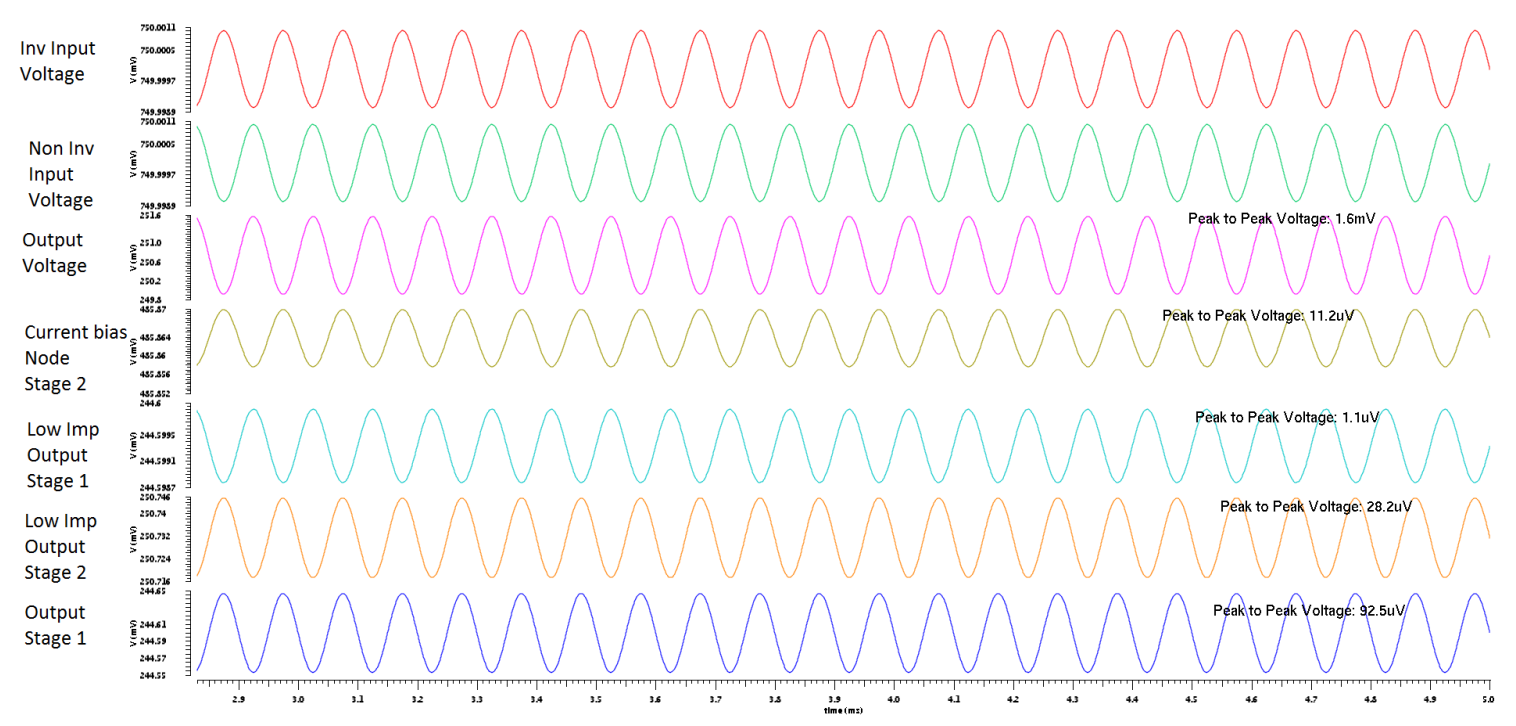

Figure 3.13: From top to bottom: Inverting input voltage signal with a peak to peak of $2 \mu \mathrm{V}$, Non-inverting input voltage signal with a peak to peak of $2 \mu \mathrm{V}$, Output voltage signal with a peak to peak voltage of $1.6 \mathrm{mV}$, Low impedance output voltage signal, Output voltage signal with a peak to peak voltage of $1.6 \mathrm{muV}$, Voltage signal at the current bias transistor drain of the second stage, Low impedance output of the first stage, Low impedance output of the second stage, High impedance output of the first stage with a peak to peak voltage of $92.5 \mu \mathrm{V}$.

To sum it up, the two factors that preserve the working of the transistor and keep the gain of the stage high are: 
a. The small signal on the ${ }^{\prime} V_{-} c s_{\_} 2^{\prime}$ node and its phase which is out-of-phase with the phase of the low impedance output signal from the first stage.

b. The current mirror load cause equal currents in both the branches of the differential gain stage. This ensues into doubled output current resulting in doubled output voltage.

\subsubsection{Differential Gain}

Small signal analysis of a generic differential amplifier reveals that the small signal gain of a differential amplifier is dependent upon the transconductance $\left(g_{m}\right)$ of the differential pair transistor and the output impedance of the stage.

$$
A_{V}=g_{m} \cdot R_{\text {out }}
$$

where $R_{\text {out }}$ is the output impedance of the output half-cell differential gain and $g_{m}$ is the transconductance of the input MOSFET transistor.

The transconductance of a transistor is given by

$$
g_{m}=\frac{\delta I_{d s}}{\delta V_{g s}}
$$

which is

$$
g_{m}=\mu_{n, p} C_{o x}\left(\frac{W}{L}\right)\left|\left(V_{g s}-V_{t n, p}\right)\right|
$$

where $\mathrm{W}, \mathrm{L}$ are width and length of a transistor, $\mu_{n, p}$ is mobility of electrons or holes depending on NMOS or PMOS transistor, $V_{g s}$ is the gate-source voltage of the transistor, $C_{o x}$ is the gate capacitance and $V_{t n, p}$ is the threshold voltage of an 
NMOS or PMOS transistor.

The above equations highlight the relationship between differential gain and the bias current in the differential pair transistors. Increase in current in the differential pair transistors increase the gain by increasing the $g_{m}$ of the transistors.

The gain of the transistors may be improved by increasing the quiescent current but the power consumption of the circuit goes up.

Increase in current drive of a transistor of a given width and length warrants increase in the overdrive voltage of the transistor, $V_{O D}$. The increase in overdrive voltage causes the drain to source voltage, $V_{D S}$, to be increased to keep the transistor in saturation. This is a visible effect in the active loads of a differential pair stage. The increase in $V_{D S}$ of the load transistors mark reduced output voltage swing.

In order to keep the power consumption of the transistor as low as possible and have a wide output voltage swing and minimum layout area the quiescent current in each branch of the differential pair stage is designed to be $\sim 5 \mu A$. Hence, a total of $\sim 10 \mu A$ is supplied by the current bias PMOS transistors.

A $1 \mu V$ differential input is applied with a common mode DC bias of $750 \mathrm{mV}$. The frequency of the inputs applied to the inverting and non-inverting input terminals of the op-amp is $10 \mathrm{kHz}$. The positive power supply voltage, $V_{D D}$, is $1.5 \mathrm{~V}$. The reference power supply voltage, $V_{S S}$ is $0 \mathrm{~V}$. 

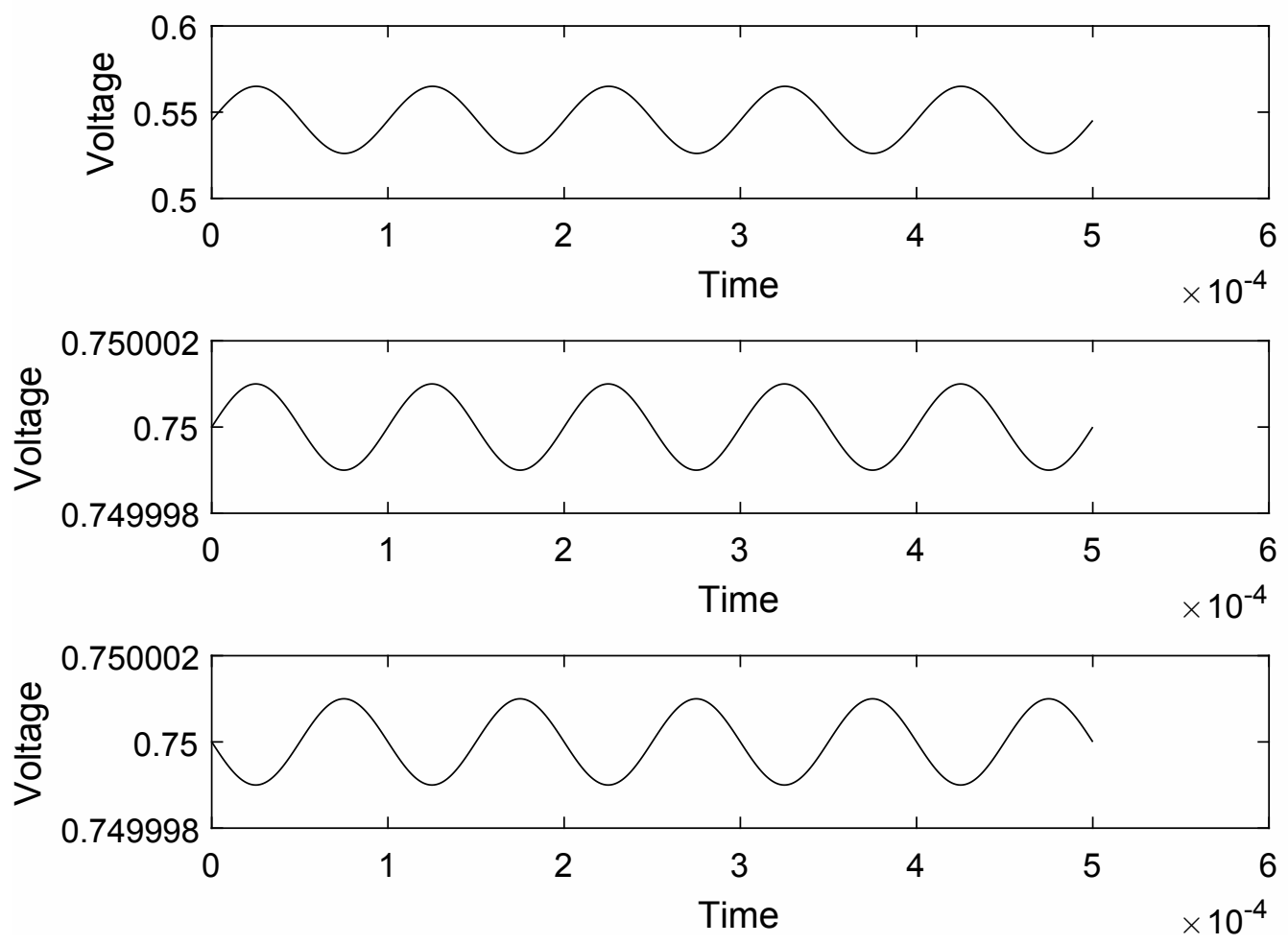

Figure 3.14: From top to bottom: Output signal produced by the op-amp which has a peakto-peak voltage of $38.8 \mathrm{mV}$ at a frequency of $10 \mathrm{kHz}$, Non-inverting voltage input signal of peakto-peak amplitude $2 \mu \mathrm{V}$ at a frequency of $10 \mathrm{kHz}$, Inverting voltage input signal of peak-to-peak amplitude $2 \mu \mathrm{V}$ at a frequency of $10 \mathrm{kHz}$ which is $180^{\circ}$ in phase with non-inverting input. 
A differential input of $2 \mu \mathrm{V}$ peak-to-peak voltage amplitude at a common mode DC bias of $750 \mathrm{mV}$ and frequency of $10 \mathrm{kHz}$, produces an output voltage of peakto-peak amplitude $38.8 \mathrm{mV}$ at a frequency of $10 \mathrm{kHz}$. Therefore, the open loop gain, $A_{D}$, of the op-amp is

$$
A_{D}=20 \log \frac{38.8 m V}{2 \mu V}
$$

Therefore,

$$
A_{D}=85.7 d B
$$

The differential gain of an operational amplifier can be increased by increasing the gain of the differential input gain stage(s) and/ or common source stage. In either cases, the gain is increased by increasing the transconductance of the input MOSFET transistors or increasing the output impedance of the transistors to increase the overall output impedance.

Increasing the transconductance involves increasing the overdrive voltage and/ or $(\mathrm{W} / \mathrm{L})$ ratio of the transistor. An increase in overdrive voltage may aid in matching the devices in case of a differential pair but the drain-source voltage has to be increased for the transistors to operate in saturation region. In this situation, inadequate current bias transistor sizes and the load transistor sizes can lead to reduction in the output common mode range of the operational amplifier.

The approach to increase (W/L) ratio of the transistor to improve the transconductance, is limited by the fact that after a certain point the output impedance of the transistor reduces with increase in $(\mathrm{W} / \mathrm{L})$ ratio, reducing the output input of the stage further. A careful and precise design entails in both cases.

A high differential gain reduces the effect of non-linearity in the components at 
the output and also aids in performance of the current source circuit and the instrumentation amplifier circuit.

\subsubsection{Common Mode Rejection}

The preceding discussion sheds lights on the differential gain of the op-amp. Equally important is the common mode gain, or common mode rejection, of the op-amp.

The common mode gain, $A_{C}$, of an op-amp is the gain factor of the common input signal. Mostly, the op-amps are designed to reject the common mode component present in the input signal and amplify only the differential component present in the input signals applied. Therefore, the common mode signal present in the input is attenuated and common mode gain is a form of quantification of the attenuation experienced by the common mode input signal.

Re-using the differential gain test bench and applying inputs of same phase results in an attenuated signal at the output of the op-amp.

Inverting and non-inverting input signals fed to the op-amp have $2 \mathrm{mV}$ peak-topeak voltage amplitude with same phase and frequency. The frequnecy of the signals is $10 \mathrm{kHz}$. The DC bias of both the input signals is $750 \mathrm{mV}$. The positive and reference power supply voltages are $1.5 \mathrm{~V}$ and $0 \mathrm{~V}$.

A common mode input of $2 \mathrm{muV}$ peak-to-peak voltage amplitude at a common mode DC bias of $750 \mathrm{mV}$ and frequency of $10 \mathrm{kHz}$, produces an output voltage of peak-to-peak amplitude $7.6 \mu \mathrm{V}$ at a frequency of $10 \mathrm{kHz}$. Therefore, the common mode gain, $A_{C}$, of the op-amp is

$$
A_{C}=20 \log \frac{7.6 \mu V}{2 m V}
$$



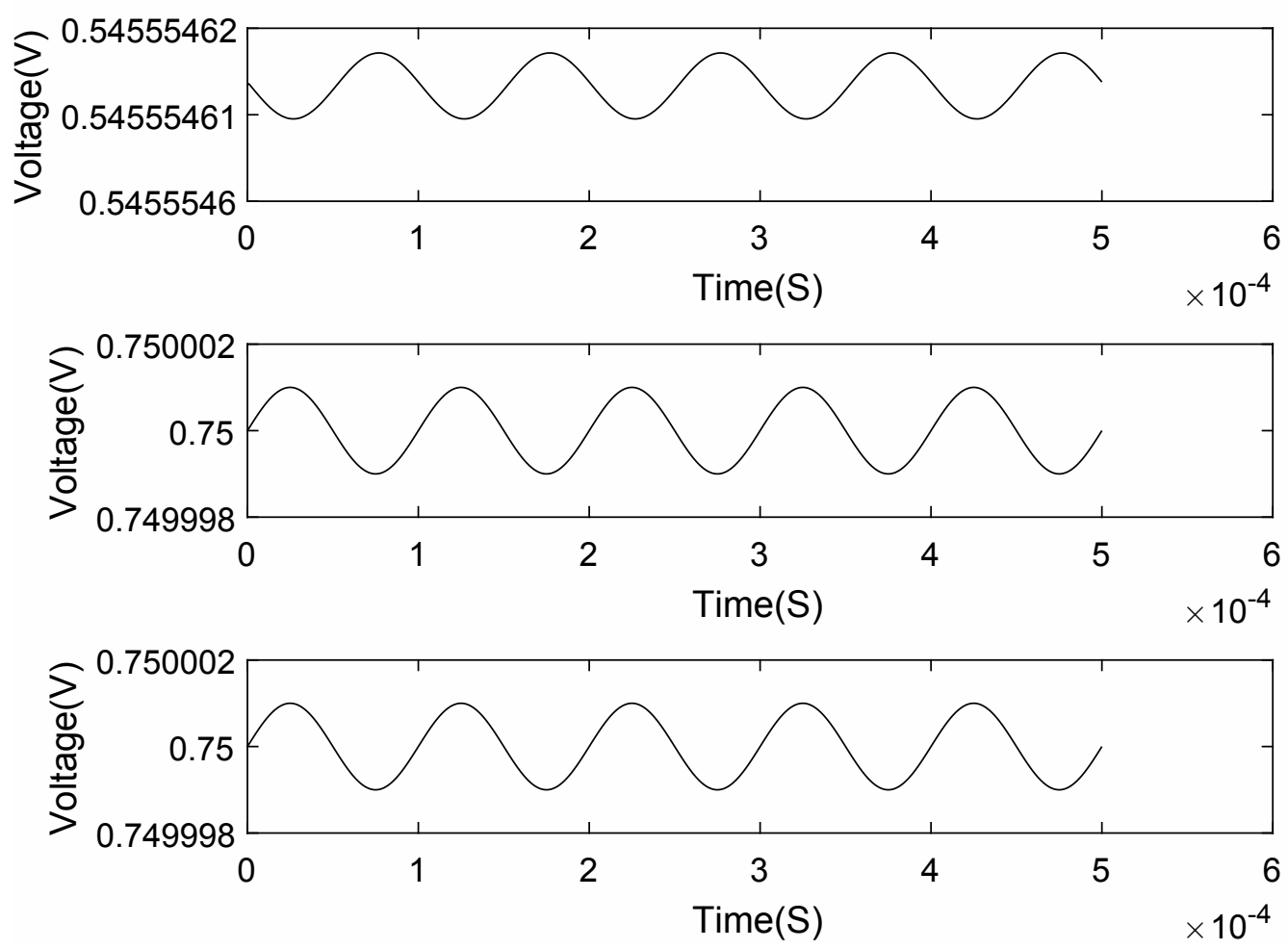

Figure 3.15: From top to bottom: Output signal produced by the op-amp which has a peakto-peak voltage of $7.6 \mu \mathrm{V}$ at a frequency of $10 \mathrm{kHz}$, Non-inverting voltage input signal of peakto-peak amplitude $2 \mathrm{mV}$ at a frequency of $10 \mathrm{kHz}$, Inverting voltage input signal of peak-to-peak amplitude $2 \mathrm{mV}$ at a frequency of $10 \mathrm{kHz}$ which has the same phase as non-inverting input

Therefore,

$$
A_{C}=-48.4 d B
$$

The common mode gain of the operational amplifier can be improved by decreasing the output impedance of the current bias transistor of the differential gain stage and/ or decreasing the transconductance of the load transistor.

By increasing the $(\mathrm{W} / \mathrm{L})$ ratio the output impedance of the current bias transistor can be reduced. Howover, keeping the gate-source voltage constant, an increase in $(\mathrm{W} / \mathrm{L})$ ratio of a transistor increases drain current implying change in bias currents in the branches of the stage. Change in bias current in the branches ensue change 
in operating point of the differential pair input MOSFET transistors.

\subsubsection{Common Mode Rejection Ratio}

Common Mode Rejection Ratio (CMRR) is the measure of an op-amp's ability to reject common mode input signals and amplify differential input signals.

Mathematically, common mode rejection ratio of an op-amp is the ratio of differential gain $\left(A_{D}\right)$ of the op-amp to the common mode gain $\left(A_{C}\right)$.

$$
C M R R=\frac{A_{D}}{A_{C}}
$$

Applying logarithm and multiplying by 20 on both sides of the equation 3.3 results in

$$
C M R R(d B)=20 \log A_{D}-20 \log A_{C}
$$

Differential gain and common mode gain are presented in sections 3.1.4 and 3.1.4 respectively.

The differential gain of the op-amp, $A_{D}$, is

$$
A_{D}=85.7 d B
$$

The common mode gain, $A_{C}$, is

$$
A_{C}=-48.4 d B
$$

Therefore, from equation 3.4, the common mode rejection ratio, CMRR, is

$$
C M R R(d B)=85.7-(-48.4)=134.1 d B
$$


Increase in differential gain and/ or rejection of the operational amplifier increases common mode rejection ratio, CMRR, of the circuit. A high CMRR is a very much desired characteristic of an operational amplifier not only because it rejects noise and improves signal-to-noise ratio, but also improves the linearity of the circuit.

\subsubsection{Output Impedance}

Ideally, output impedance of an op-amp is zero, but in practice it is finite and is desired to be a low value.

The output impedance of an op-amp is set by the final stage of the op-amp and in this case the final stage is a buffer, which is typically added to the op-amp to drive resistive loads.

A buffer stage is a common drain amplifier whose gain is lower than 1 . The buffer stage is not only reduces the output impedance of an op-amp but also drive large currents and adjusts the DC bias of the op-amp.

The output stage is usually designed by using NMOS transistors because, for a given width and length, the current handling capability of an NMOS transistor is higher than its PMOS transistor counterpart. Conversely, for a given current value, the width and length requirements of an NMOS transistor is lower than its PMOS counterpart. Hence, NMOS transistors are used to design the buffer stage for the op-amp.

When DC analysis is performed with a $750 \mathrm{mV}$ common mode DC input at the input terminals of the operational amplifier, the output conductivity of the buffer stage transistors, T22 and T21, is found to be $15.1 \mu \mathrm{A} / \mathrm{V}$ and $15 \mu \mathrm{A} / \mathrm{V}$ respectively. 
Therefore, the open loop output impedance of the operational amplifier is

$$
Z_{\text {output }}=r_{d s 2} \| r_{d s 1}=\frac{1}{g_{d s 1}+g_{d s 2}}=\frac{1}{15.1 \mu+15 \mu}=33.3 \mathrm{~K} \Omega
$$

The output impedance is high and increased as a result of reducing the $(\mathrm{W} / \mathrm{L})$ ratio of both the transistors to adjust the output voltage at mid-rail.

The open loop output impedance of an op-amp can be reduced by increasing the width of the transistors keeping the length constant. This reduces the output impedance of individual transistors ensuing in reduced output impedance. The downside to increasing the width of the transistors is increased current consumption in the buffer stage and increased layout area. Also, increased current reduces reduces the gain of the stage which reduces the overall gain of the op-amp.

\subsubsection{Input and Output Common Mode Range}

Operational amplifiers have finite input and output voltage range.

The finite range of voltage at the input of the op-amp is referred to as input common mode range, and similarly the voltages between the maximum and minimum voltage at the output is referred to as output common mode range or output swing.

\section{Input Common Mode Range}

The Input Common Mode Range (ICMR) is the allowable range of input voltages within which the load transistors, current source transistors and differential pair transistors operate in saturation region, for which op-amp operates normally.

The figure 3.16 shows the first differential gain stage of the op-amp. In the figure, input common mode range is the common mode DC input range that can applied 
to input MOSFET transistors which to ensure that all the transistors in the circuit operate in saturation region.

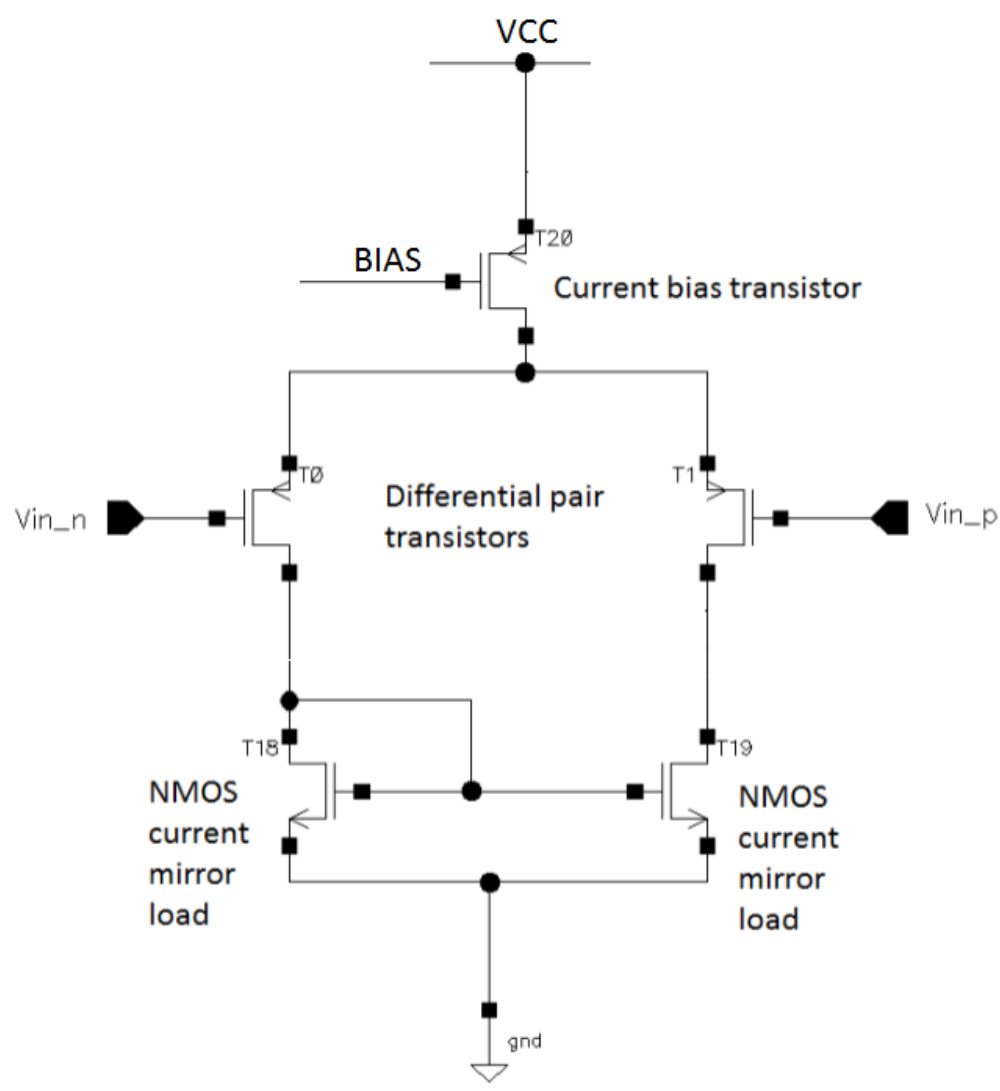

Figure 3.16: The figure shows the first differential input gain stage of the operational amplifier. Transistor T20 is the current bias transistor which is biased by a voltage that is obtained from the bias stage of the operational amplifier. $\mathrm{T} 1$ and $\mathrm{T} 0$ are differential pair input MOSFET transistors that act as common source amplifiers. T18 and T19 NMOS transistors act as current mirror load.

From the figure 3.16, the upper limit for the input common range is dictated by the drain-source voltage of the current bias transistor. If the gate-source voltage of the current bias transistor is a fixed $V_{\text {bias }}$. Then, the minimum drain-source voltage of the transistor is $\left(V_{b i a s}-V_{T p}\right)$.

Having reduced the upper input range by $\left(V_{\text {bias }}-V_{T p}\right)$, another limitation comes 
from the gate-source voltage of the differential input MOSFET transistors, T0 or T1. The differential input MOSFET's require a minimum gate-source voltage to source the bias current in the branch. Therefore, assuming the minimum gatesource voltage of the differential input MOSFET T0 is $V_{g s 0}$, the maximum input common mode voltage is calculated to be

Maximum common mode input voltage $=V_{C C}-\left(V_{\text {bias }}-V_{T p}\right)-V_{g s 0}$

Usually, the lowest common mode input voltage is dictated by the drain-source voltage of the load transistors. However, in this case the load is a current mirror load. The diode-connection ensures that the transistor is in saturation for as long as the transistor gate-source voltage is greater than threshold voltage of the transistor. This increases the dynamic range of the input.

The figure 3.17 illustrates DC simulation output voltage versus input common mode voltage plot. For a single power supply of $1.5 \mathrm{~V}$ and $0 \mathrm{~V}$ ground reference, sweeping the input common mode input from $0 \mathrm{~V}$ to $1.5 \mathrm{~V}$, the input common mode range is found to be $\sim(2 \mathrm{mV}, 1 \mathrm{~V})$. 


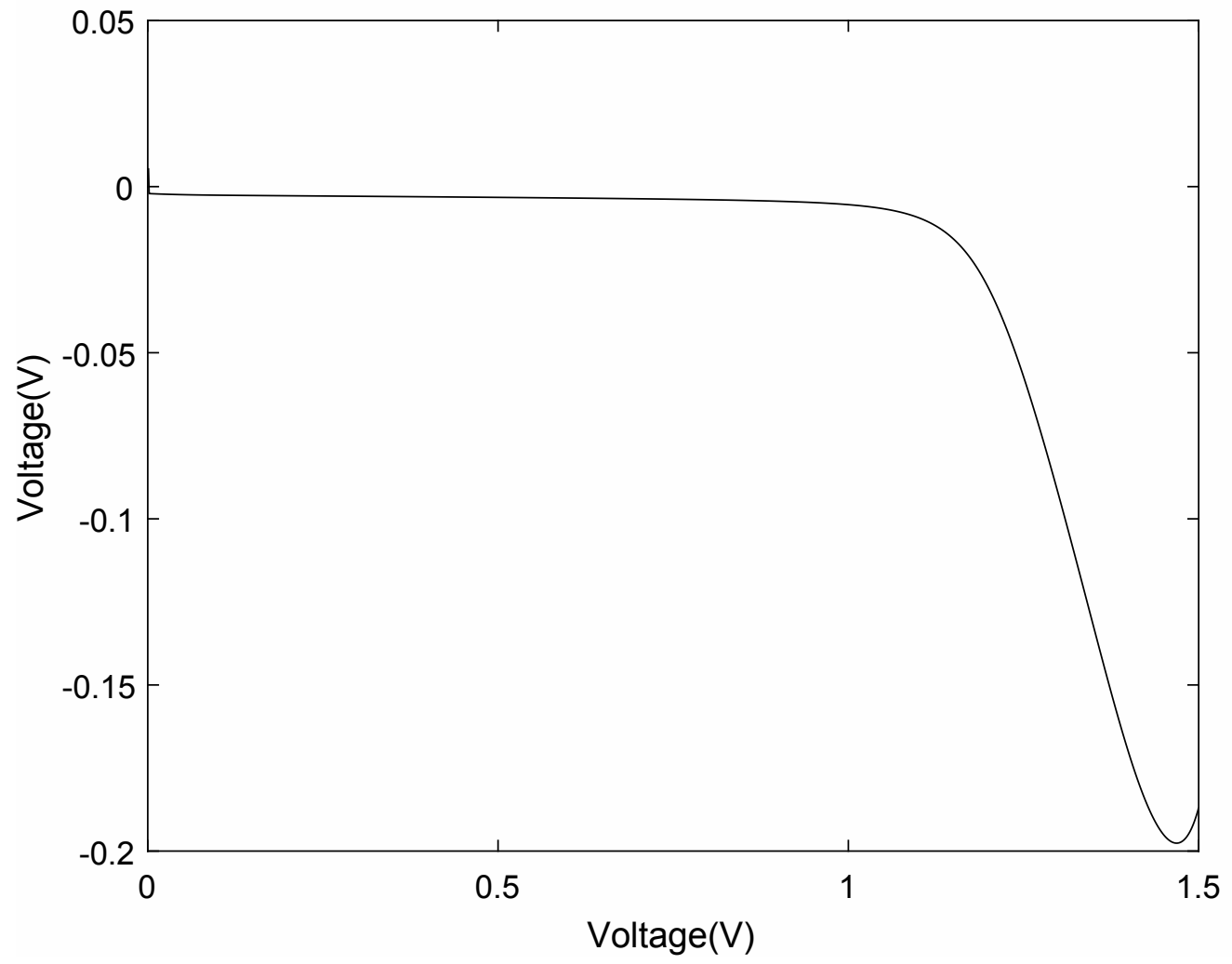

Figure 3.17: The figure illustrates, DC analysis plot of output voltage of the operational amplifier plot versus common mode input voltage. The range of common mode input is $(2 \mathrm{mV}$, $1 \mathrm{~V})$. 


\section{Output Common Mode Range}

The output common mode range or output voltage swing is the output voltage range with finite maximum and minimum voltages beyond which the one or more transistors in the op-amp are no longer is operating in saturation region.

If the output voltage swings close to the power supply voltages then the op-amp is said to exhibit 'rail-to-rail' output. There is no industry standard rail-to-rail spec which dictates the output voltage swing for specified power supply voltages. Therefore, each company has their own standard for output voltage swing.

From the figure 3.16, the output voltage is limited by the drain-source voltage of the current bias transistor, T20, and the load transistors of an op-amp, T18 and T19.

Subjecting the operational amplifier to DC analysis with a common mode input of $750 \mathrm{mV}$, the drain-source voltage of the T20 transistor is $\sim 505 \mathrm{mV}$. And, the drain-source voltage of the load transistors is $\sim 250 \mathrm{mV}$. In the figure 3.16 , though the drain-source of the diode-connected MOSFET, T18, is $250 \mathrm{mV}$, the output is acquired from the drain of T1. Since the T19 transistor is not a diode-connected transistor, the output voltage can swing an additional voltage equal to the threshold voltage of the NMOS transistor.

Therefore, the output common mode voltage range of the circuit is $\left(V_{C C}-0.5 \mathrm{~V}\right.$, $\left.V_{S S}+0.25 V-V_{T n}\right)$.

The output plot versus common mode DC input in the 3.18, displays the output voltage swing of $75 \mathrm{mV}$ to $1 \mathrm{~V}$. Therefore, obtaining voltage values of the node from DC simulations, the input common mode range, ICMR, of the op-amp is

$$
I C M R=(2 m V, 1 V)
$$



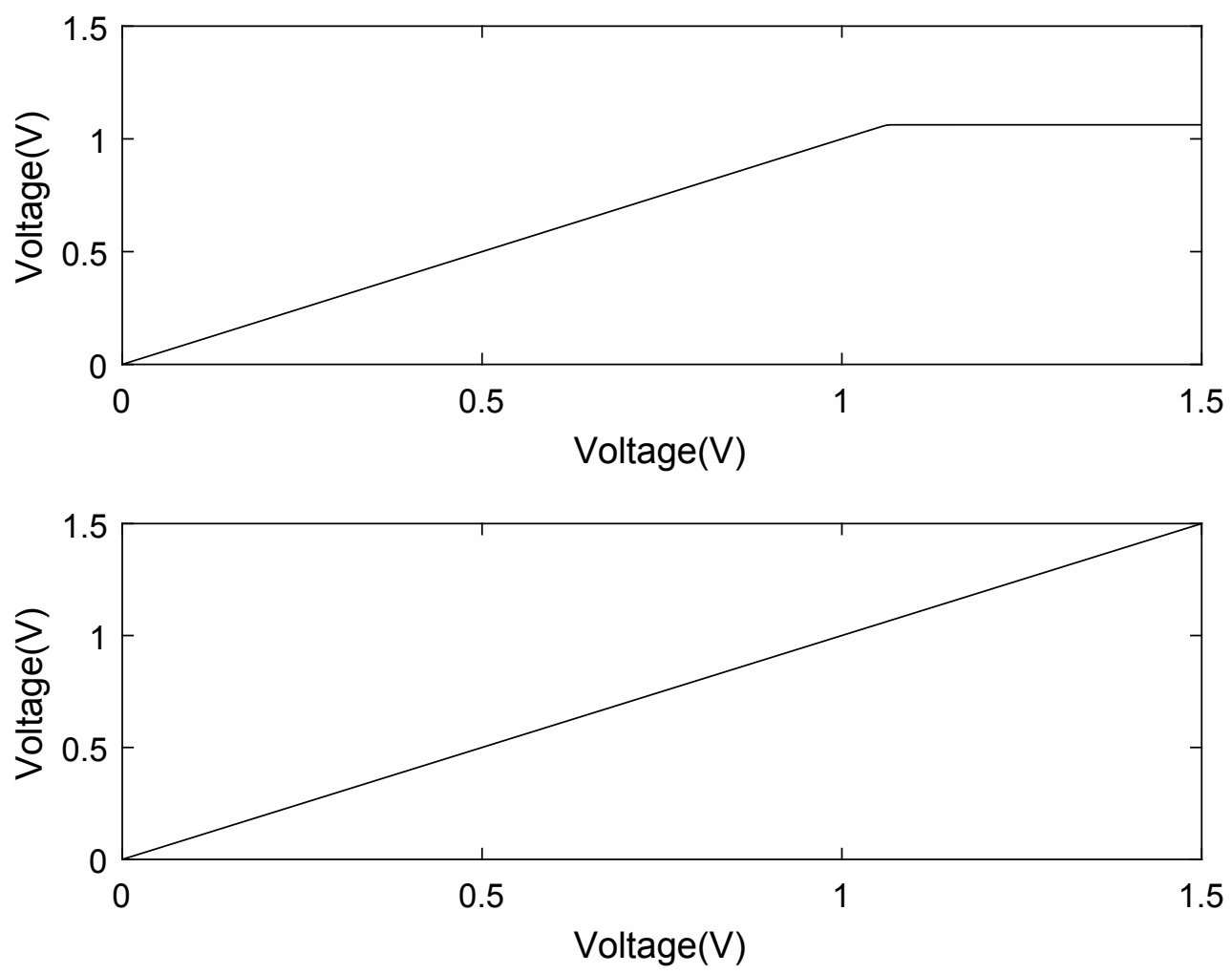

Figure 3.18: In the figure, if the operational amplifier is configured as a buffer and when the input common mode voltage (red) is swept from $0 \mathrm{~V}$ to $1.5 \mathrm{~V}$, the output (green) follows the input until $\sim 1 \mathrm{~V}$ common mode input. The output voltage swing is observed to be $75 \mathrm{mV}$ to $1 \mathrm{~V}$.

and the output common mode range, OCMR, of the op-amp for a $1.5 \mathrm{~V}$ and $0 \mathrm{~V}$ power supply voltages is

$$
O C M R=(75 m V, 1 V)
$$

With regards to the bioimpedance system, a large input voltage range to inject desired current into the biological subject. A large input voltage range makes it possible to inject large currents without reducing the gain setting resistor in the current source.

A wide output voltage swing is required to acquires large voltage response signal if the injected current is large. Although the it is totally dependent upon the 
requirments, generally a wide output voltage swing is desired.

\subsubsection{Slew Rate}

Slew rate of an op-amp is defined as the rate of change of output voltage expressed in $\frac{V}{s}$ or $\frac{V}{\mu S}$.

Mathematically, it is expressed as

$$
\text { Slew } \operatorname{rate}(S R)=\frac{d V_{\text {out }}}{d t}
$$

In the figure 3.19 , the output voltage of the operational amplifier and input voltages are shown by performing transient simulations. 


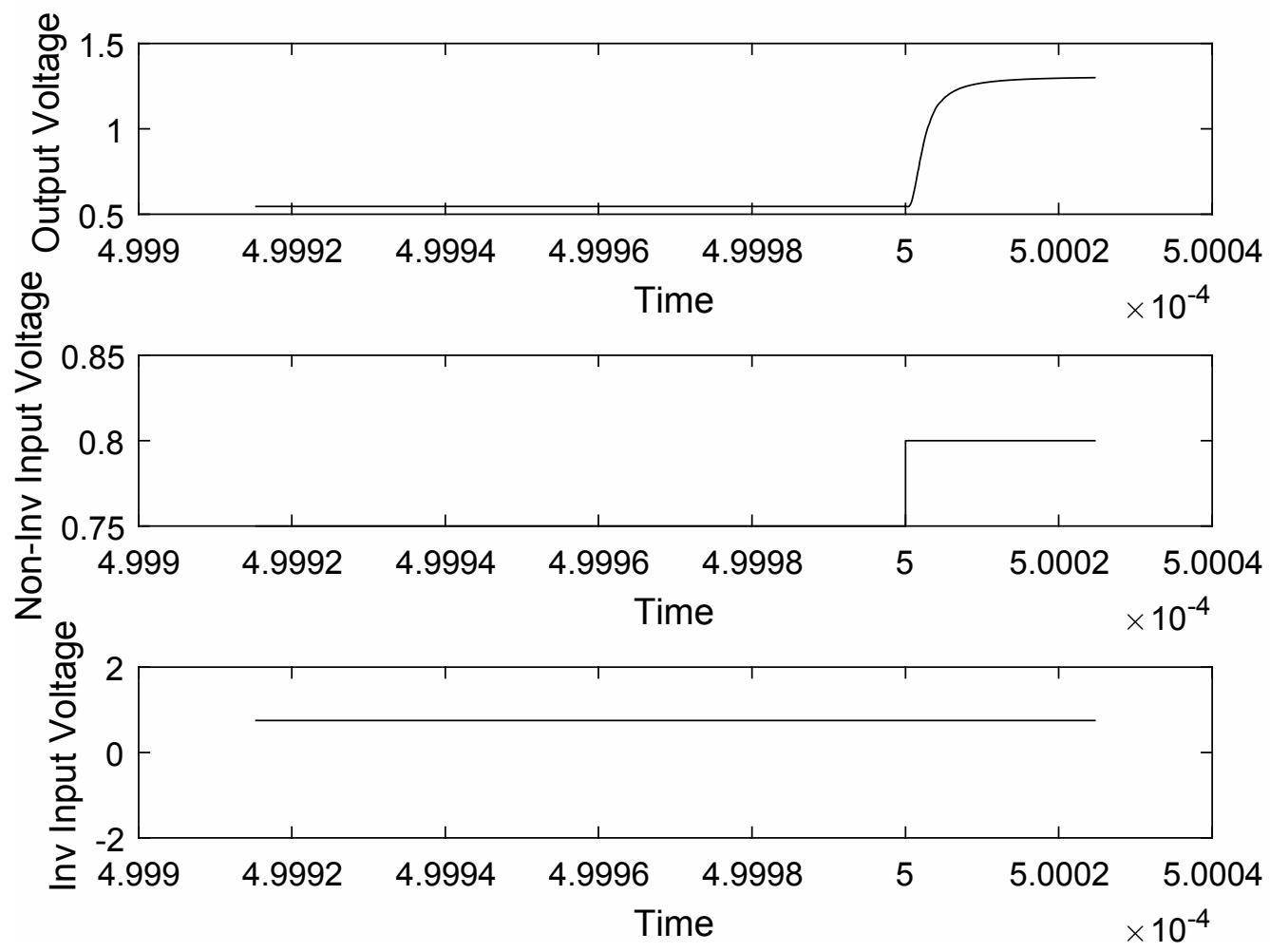

Figure 3.19: From top to bottom: Output voltage signal changes from $544.3 \mathrm{mV}$ to $1.29 \mathrm{~V}$ in 15.2ns, input voltage at non-inverting terminal changes from $750 \mathrm{mV}$ to $800 \mathrm{mV}$, input voltage at inverting terminal is a constant $\mathrm{DC}$ of $750 \mathrm{mV}$. The slew rate of the operational amplifier is observed to be $49 \mathrm{MV} / \mathrm{s}$. 
Typically, slew rate is determined from the ratio of change in output voltage from $10 \%$ to $90 \%$ of the final output to the time taken for the change.

Therefore, from output voltage signal seen in the figure 3.19, the slew rate is found to be

$$
\text { Slew rate }=\frac{1.29 \mathrm{~V}-544.3 \mathrm{mV}}{15.2 \mathrm{~ns}}=49 \mathrm{MV} / \mathrm{s}
$$

The slew rate in case of the thesis does not play a major role because the circuit is operated in low frequencies.

\subsubsection{Non-linearity}

Output of a system $\mathrm{Y}$ for an input $\mathrm{X}$ are said to bear a linear relationship if they follow the relationship shown in the equation 3.11 .

$$
Y=m X+C
$$

Assuming $\mathrm{C}$ is 0 , output $\mathrm{Y}$ is said to obey principle of superposition if its dependency on the input $\mathrm{X}$ is as shown in the equations 3.12, 3.12 and 3.14.

$$
\begin{aligned}
& Y_{X_{1}}=m X_{1} \\
& Y_{X_{2}}=m X_{2}
\end{aligned}
$$

then

$$
Y_{X_{1}+X_{2}}=m\left(X_{1}+X_{2}\right)=Y_{X_{1}}+Y_{X_{2}}
$$

For a slope of $1, \mathrm{~m}=1$, and assuming the constant is $0, \mathrm{c}=0$, the output, $\mathrm{y}$, of a linear system versus input, $\mathrm{x}$, is shown in the figure 3.20. 


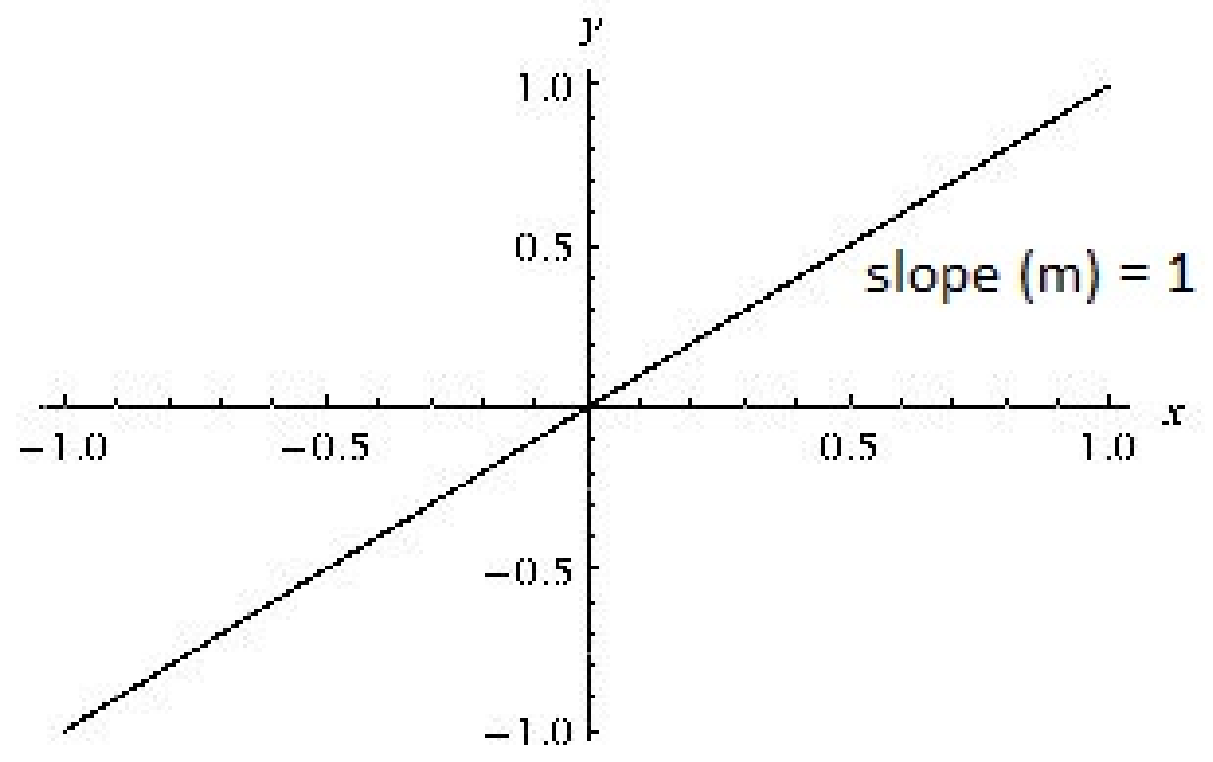

Figure 3.20: The figure illustrates the dependency of output of a system upon its input if the system in with 'y' as its output and 'x' as its input

Having defined linearity, non-linearity of a system can be stated as deviation of the output of a system from the straight line plot of unity slope and it is illustrated in the picture 3.21 . 


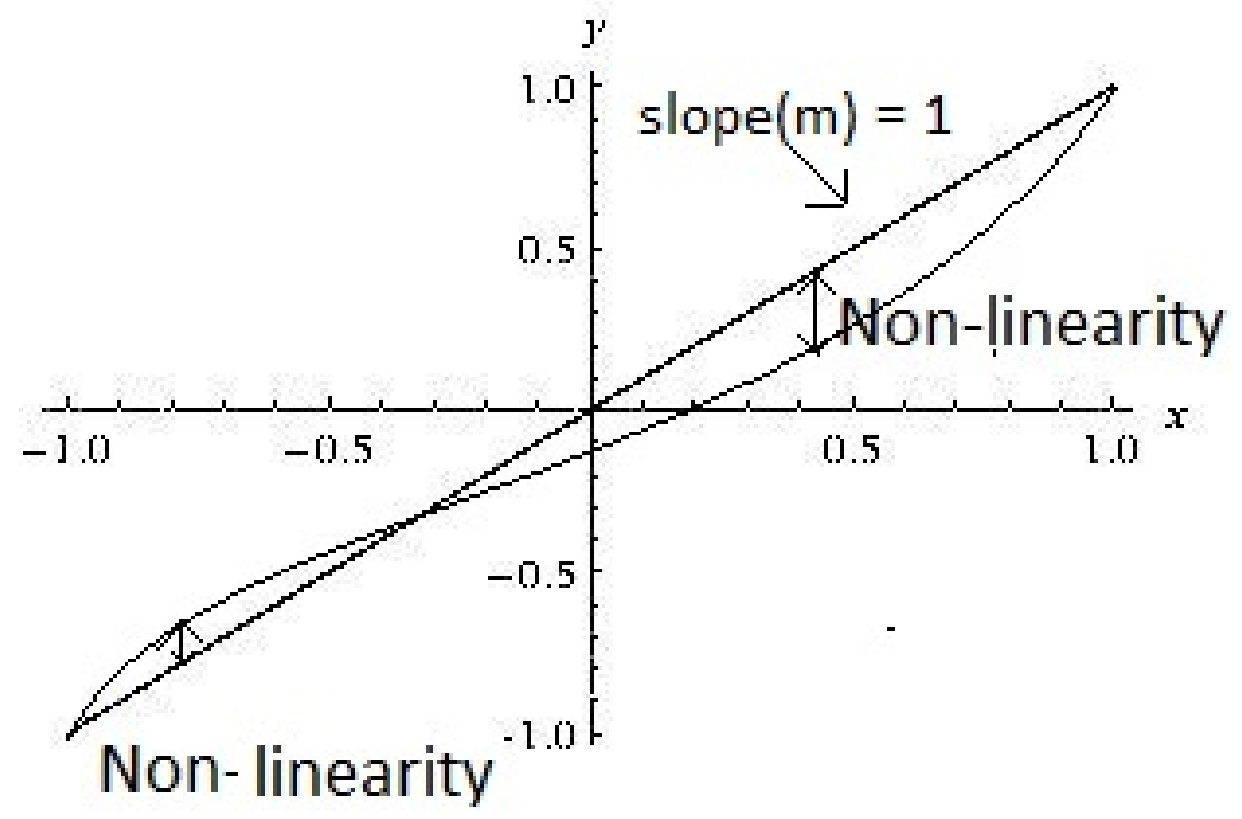

Figure 3.21: Non-linearity, as shown in this figure, is the deviation of the output from the straight line plot of slope 1 shown in the figure 
The slope of the output signal with respect to the input applied illustrates a clear picture of non-linearity. Assuming the circuit is a buffer, if the circuit is perfectly linear for all the input, then the slope of the output is 1 for all the input, as output follows input. The plot of output slope with respect to input would be a horizontal line situated at a distance of 1 unit from the $\mathrm{X}$ axis (assuming the input voltage is $\mathrm{X}$ axis). However, a perfectly linear circuit is only and idea and in reality circuits operate in non-linear regions depending on the design and inputs. Thus, in case the circuit is operating in a non-linear region the slope of the output in that region is non-unity.

Deviation of the output from linearity can be calculated from the slope as shown in the equation

$$
\text { Deviation }=\text { Input }-(\text { Input } x \text { slope obtained at that input })
$$

The figure 3.22, shows the slope of the output of a buffer with respect to common mode input voltage when swept from $0 \mathrm{~V}$ to $1.5 \mathrm{~V}$. 


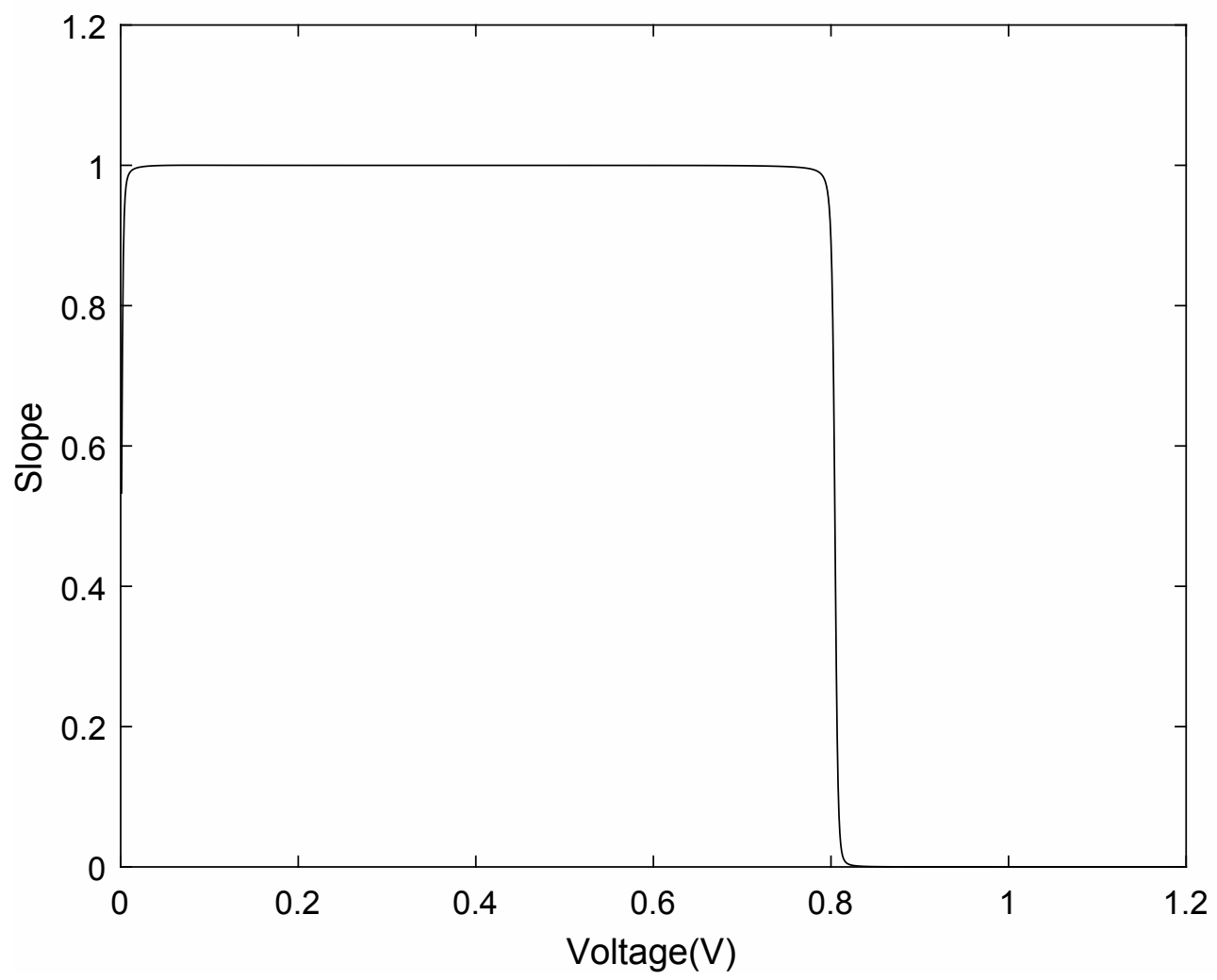

Figure 3.22: Seen in the figure clearly is the slope of the output with respect to common mode input being swept from $0 \mathrm{~V}$ to $1.5 \mathrm{~V}$. The slope of the output when the input is $75 \mathrm{mV}$ is $999.9 \mathrm{~m}$, and the slope of the output when the input is $1 \mathrm{~V}$ is $998.5 \mathrm{~m}$. The slope examined at $75 \mathrm{mV}$ and $1 \mathrm{~V}$ because the output common mode range is $75 \mathrm{mV}$ to $1 \mathrm{~V}$. 
From the figure 3.22 , it is clear that the maximum deviation is at $1 \mathrm{~V}$ common mode input and the minimum deviation is at $75 \mathrm{mV}$ common mode input. Therefore,

$$
\begin{gathered}
\text { Maximum deviation }=1-\left(\begin{array}{lll}
1 & x & 998.5 m
\end{array}\right)=1.5 m V \\
\text { Minimum deviation }=75 m V-\left(\begin{array}{llll}
75 m V & x & 999.9 m
\end{array}\right)=7.5 \mu \mathrm{V}
\end{gathered}
$$

\subsubsection{Offset Voltage}

Ideally, the output voltage has to be 0 when the magnitude of the differential input is $0 \mathrm{~V}$. However, in reality, the output voltage to deviates from $0 \mathrm{~V}$. The magnitude of the voltage deviation is known as offset voltage.

A finite amount of voltage offset between the input terminals can adjust the output voltage to $0 \mathrm{~V}$. This voltage is input referred offset voltage. Typically, the offset voltage is modeled as a source voltage at the input terminal of the op-amp.

Though the offset voltage is especially important for DC sensitive circuits, the voltage response measured by the instrumentation amplifier is the drop in voltage across the unknown impedance and the offset voltage is covered in the DC bias of the output voltage which is set by a reference input signal. Hence, the effect of offset voltage is negligible. Nevertheless, a small offset voltage is a desired design specification for an op-amp.

The designed operational amplifier is configured as a buffer and applied with inputs $750 \mathrm{mV}$. The output obtained, shown in figure 3.23 , is $749.915 \mathrm{mV}$. Therefore, the offset voltage is

$$
V_{\text {offset }}=750 \mathrm{mV}-749.915 \mathrm{mV}=85 \mu \mathrm{V}
$$



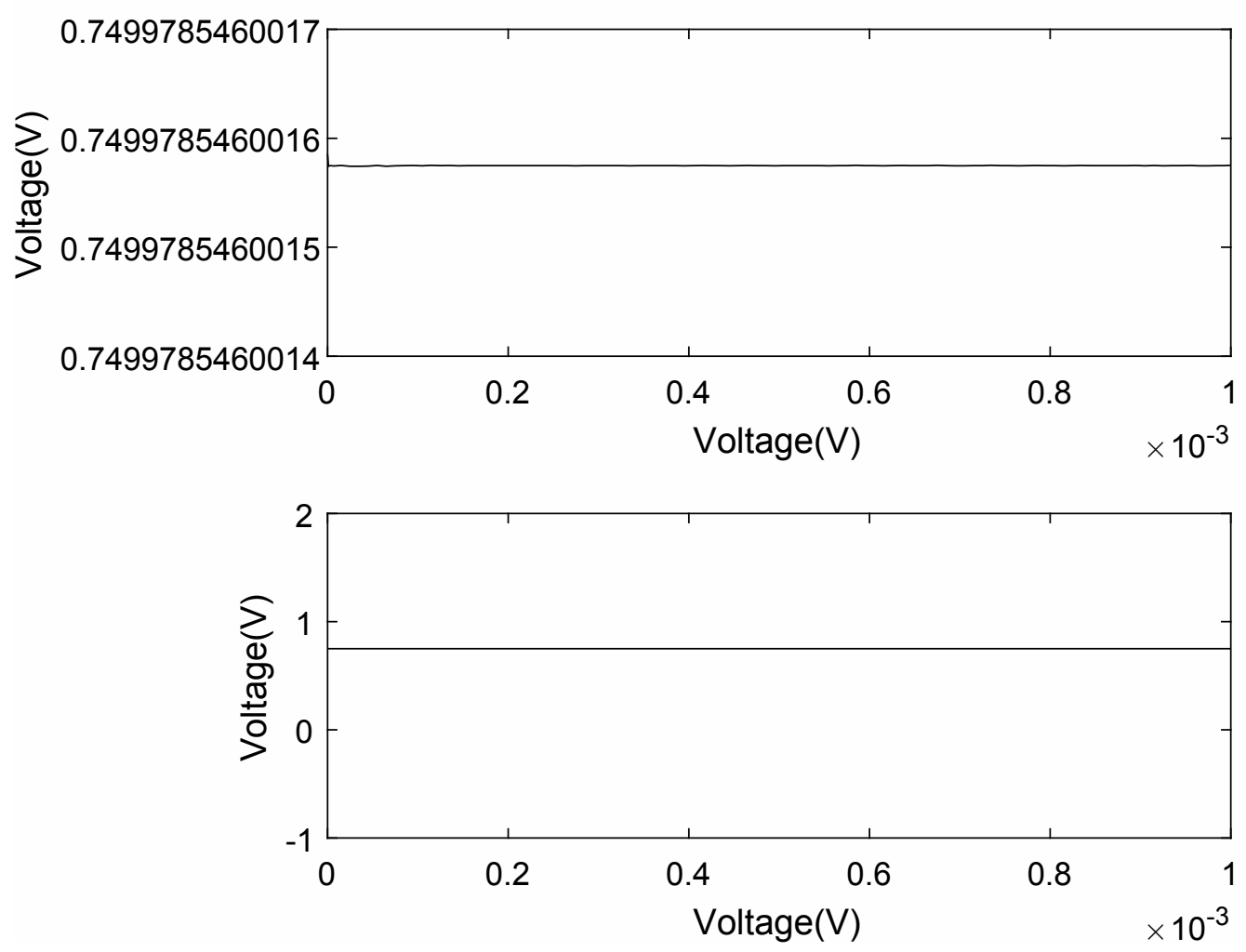

Figure 3.23: A transient analysis is performed upon a buffer circuit and the figure illustrates the results. When the input (red) to both the input terminals of the operational amplifier is a constant $750 \mathrm{mV}$ DC, the voltage output (green) is $749.915 \mathrm{mV}$. This shows that the offset voltage is $85 \mu \mathrm{V}$.

\subsubsection{Power Consumption}

The power consumption of the operational amplifier is determined from DC simulation of the circuit.

The static power consumption of the circuit is calculated by

$$
\text { Power } \text { consumption }=\text { current } \text { consumed } x \text { power supply }
$$

After performing DC analysis, for a power supply voltage of $1.5 \mathrm{~V}$, the current consumed by the operational amplifier is $113.1 \mu \mathrm{A}$.

Therefore, the power consumption of the operational amplifier is

$$
\text { Power } \text { consumption }=1.5 \mathrm{~V} \quad x \quad 113.1 \mu \mathrm{A}=169.65 \mu \mathrm{W}
$$




\subsection{Current Source}

This section details circuit description, working and simulations of basic Howland current source, modified Howland current source and mirrored modified Howland current source. While Transient simulations demonstrate the activity in the circuit when excited by a voltage input, AC simulation is performed to find the range of frequencies for which the circuit works as a current source.

Current sources are critical to the design of a bioimepdance system. It has to drive a current of constant AC magnitude regardless of the load connected to it, especially when the load is a human subject where the magnitude of the load impedance is not only dependent the frequency of operation but also other factors such as condition of the subject, tissue whose impedance is being determined (the whole body impedance is higher than the impedance of an individual part of the body), placement of electrodes, type of electrodes, etc.

It is critical that the output impedance of the current source is very high compared to the load impedance. A load which is comparable to the output impedance of the current source varies the output current of the circuit.

It is tacitly understood that the current source circuit has to be operational for the range of frequencies for which the bioimpedance is required to be performed, in that the circuit has to produce constant current for the range of frequencies that is of interest. 


\subsubsection{Basic Howland Current Source}

A basic Howland current source as seen in the Chapter 2 is an op-amp based voltage controlled current source developed Brad Howland which works on the principle of ratio-ed resistors and positive feedback bootstrapping.

The basic Howland current source designed using the op-amp with feedback resistors and input resistors is seen in the figure 3.24.

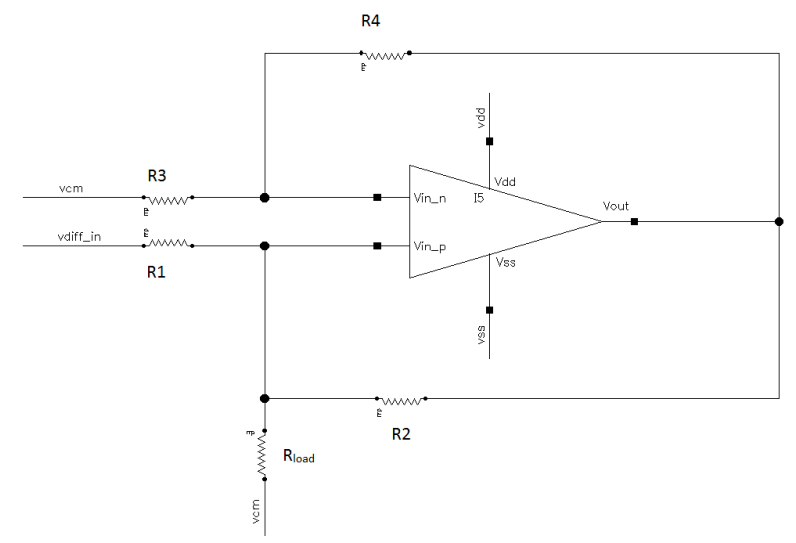

Figure 3.24: The basic Howland current source seen in the figure is designed using the op-amp discussed in the previous sections. The feedback resistors and the input resistors seen the figure are valued at $100 \mathrm{~K} \Omega$ and are used for simulation purposes only. The load resistance used

In the figure 3.24 , the feedback resistors, $R_{\text {feedback }}$, and the input resistors, $R_{\text {input }}$, are made equal and are equal to $100.1 \mathrm{~K} \Omega$.

$$
R_{\text {feedback }}=R_{\text {input }}=100.1 \mathrm{~K} \Omega
$$

The load impedance of the circuit is

$$
R_{\text {Load }}=1 K \Omega
$$


The nets labeled vdiff_in and vcm are AC input signal and DC common mode input signal respectively.

In theory, when an input is applied, vis-a-vis $v \operatorname{dif} f \_i n$, a load current is observed in the load resistor which is equal to sum of current in the input resistor and positive feedback resistor.

The following figure with plots demonstrate the transient behavior of the current source circuit when a differential input, vdiff_in of $200 \mathrm{mV}$ peak-to-peak voltage is applied. The power rails are $1.5 \mathrm{~V}$ and $0 \mathrm{~V}$. The common mode DC bias, vcm, applied is $750 \mathrm{mV}$ (mid-rail voltage).

Clearly seen in the plots of the figure 3.25 , the current in the input resistor, R1, and the feedback resistor, R2, sum up to the load current in the load resistor, $R_{\text {load }}$.

Also,

$$
I_{\text {load }, \text { peak }}=\frac{v \operatorname{diff\_ in}}{R_{1}}=\frac{100 \mathrm{mV}}{100 \mathrm{~K} \Omega}=1 \mu \mathrm{A}
$$

Since, the peak load current is $1 \mu A$, the peak-to-peak current is

$$
I_{\text {load }, \text { peak }}-\text { to- } \text { peak }=2 I_{\text {load }, \text { peak }}=2 \mu \mathrm{A}
$$

The above equations match the simulations and confirm the working of the circuit. The inverting and non-inverting terminal voltages along with the op-amp's output voltage plots presented in the figure 3.26 aid in understanding the circuit from the point of view of the voltages.

For a differential input of $200 \mathrm{mV}$ peak-to-peak voltage magnitude, the current observed in the load current is $1 \mu \mathrm{A}$. With the load resistance being $1 \mathrm{~K} \Omega$, the dif- 


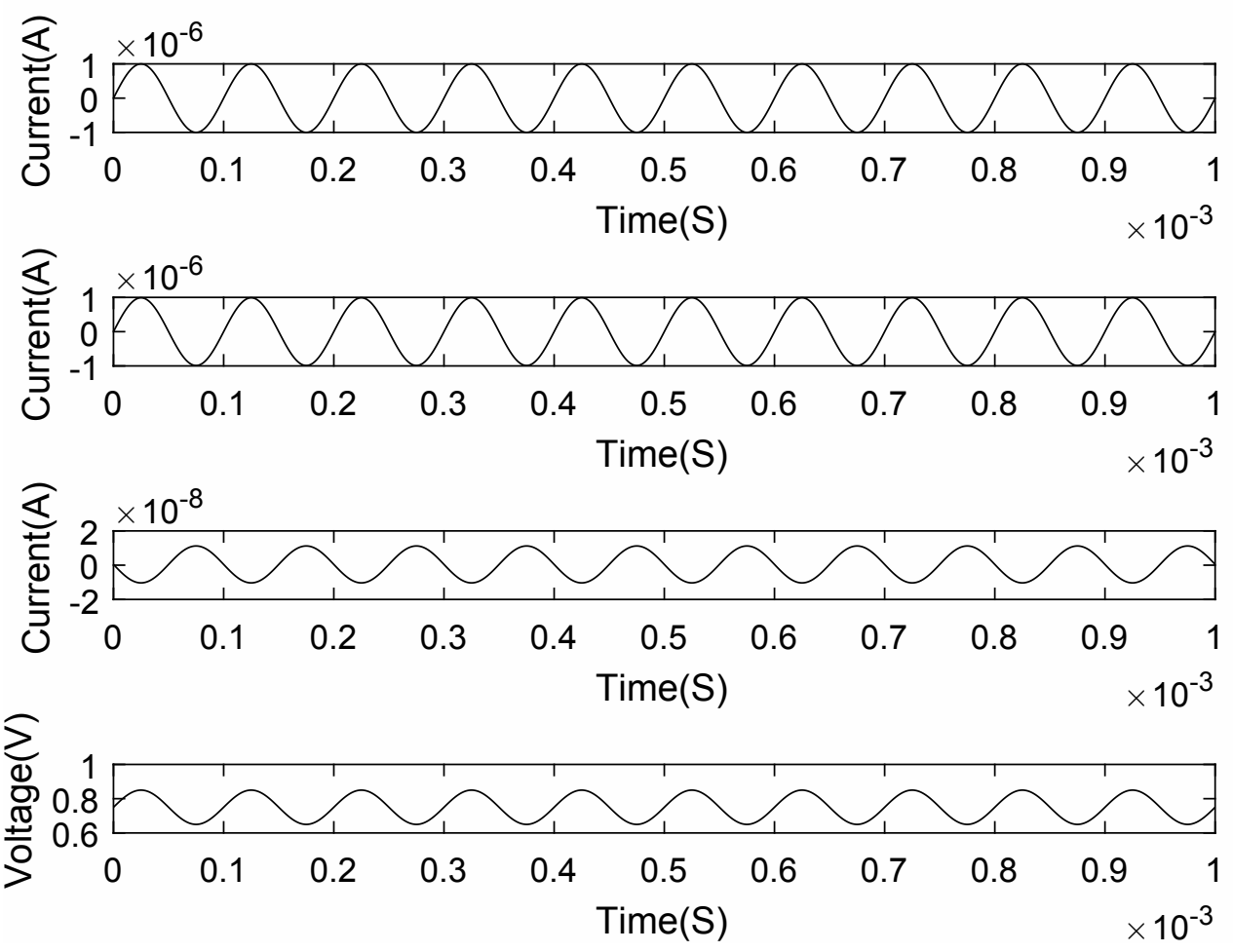

Figure 3.25: From top to bottom: $200 \mathrm{mV}$ peak-to-peak differential voltage input signal at a common mode DC of $750 \mathrm{mV}$, load current with a peak-to-peak value $\sim 2 \mu \mathrm{A}$, Current in the input resistor with a peak-to-peak value $\sim 1.97 \mu \mathrm{A}$, current in the feedback resistor with a peakto-peak value $\sim 21.5 \mathrm{nA}$. The current in the input and the feedback resistor add up to produce the load current.

ferential voltage at the non-inverting terminal of the op-amp is $1 \mathrm{mV}$. An op-amp of high gain tries to reject the difference between the inverting and non-inverting terminals ensuing in rise in the voltage ot the inverting terminal of the op-amp. Therefore, the differential voltage of $1 \mathrm{mV}$ peak voltage that originated at the noninverting terminal of the op-amp is also observed at the inverting terminal of the op-amp.

There is current proportional to the feedback resistance flowing in the feedback resistors. This current sets up the voltage at the output of the op-amp. Considering only the $\mathrm{AC}$ voltages, the op-amp output voltage is summation of the load 

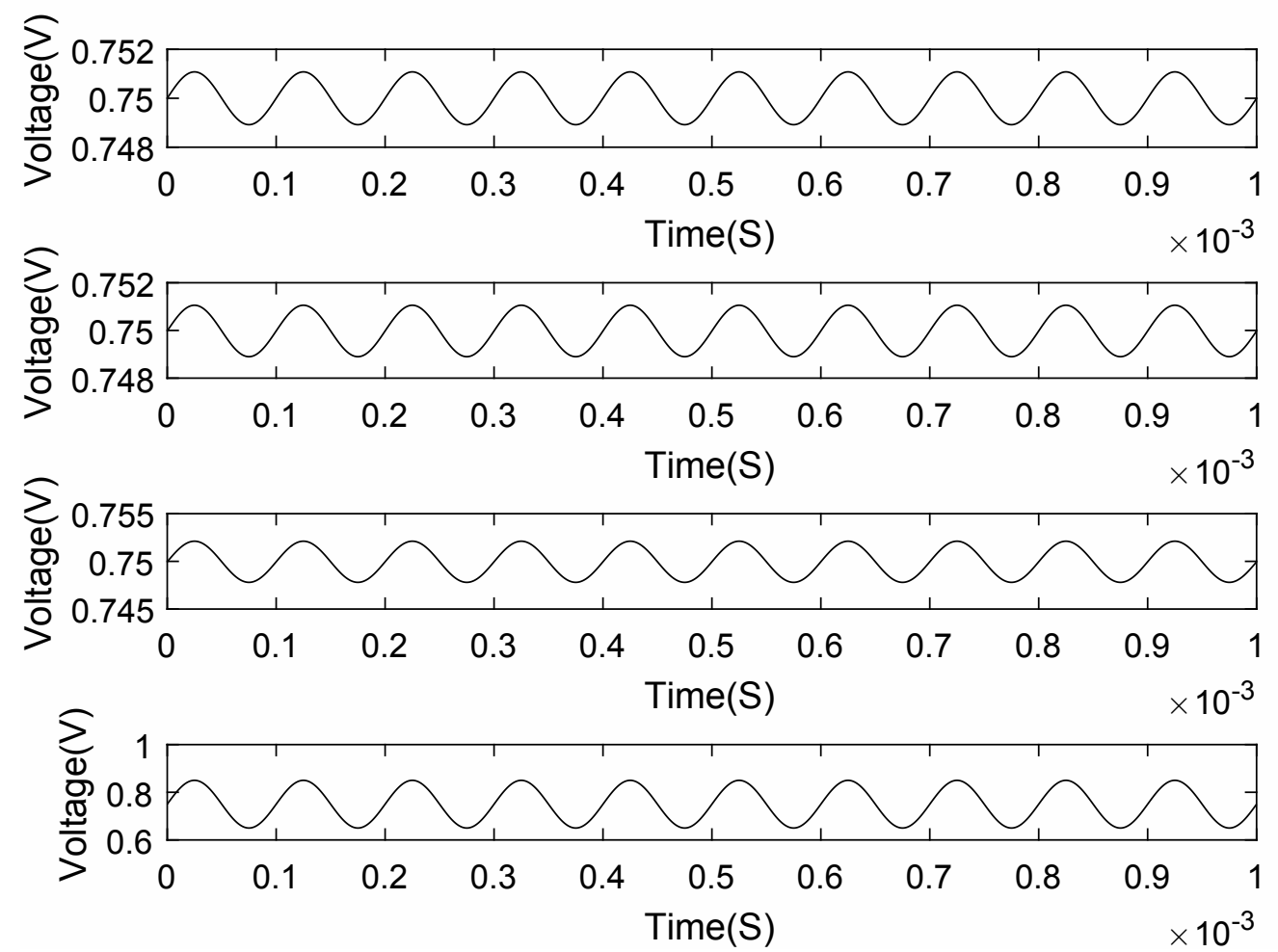

Figure 3.26: From top to bottom: $200 \mathrm{mV}$ peak-to-peak differential voltage input signal at a common mode $\mathrm{DC}$ of $750 \mathrm{mV}$, non-inverting terminal voltage plot with $2 \mathrm{mV}$ peak-to-peak voltage magnitude, inverting terminal voltage plot with $2 \mathrm{mV}$ peak-to-peak voltage magnitude, Op-amp's output voltage with $4 \mathrm{mV}$ peak-to-peak voltage magnitude

voltage and voltage across the feedback resistor.

$$
V_{o}=V_{L}+R_{2} \cdot I_{f}=1 m V+10 n .100 K \Omega=2 m V
$$

The transient behavior of the basic Howland current source is verified from the simulations.

While the transient behavior of the circuit checks out, it is important that the current source produces current for the range of frequencies that are of interest. The following plot is current versus frequencies which provides the bandwidth of the circuit.

The current vs. frequency plot shown in figure 3.27 clearly shows that the current 


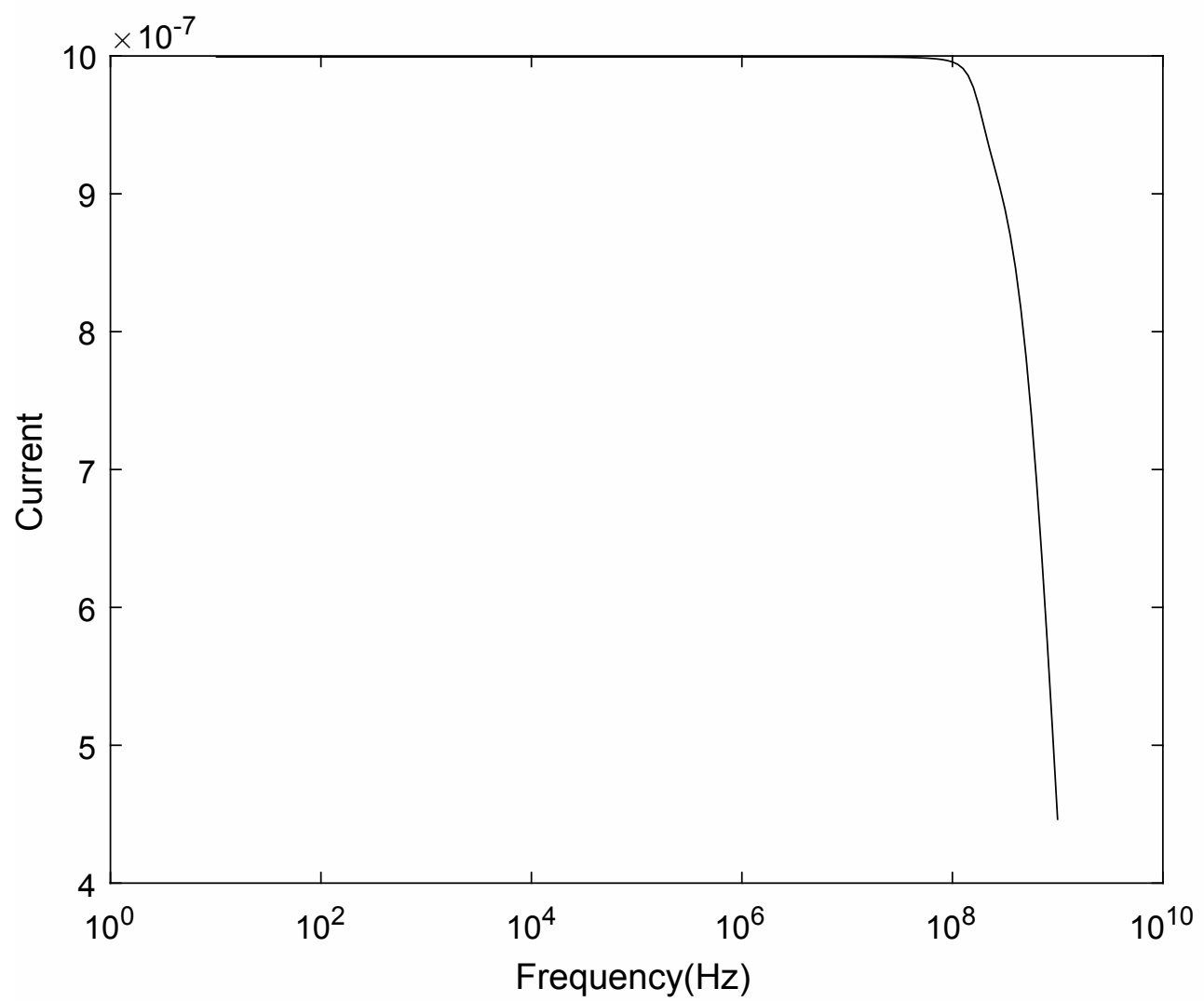

Figure 3.27: The figure illustrates the current vs. frequency curve of the current source. The current output is $999.2 \mathrm{nA}(\sim 1 \mu \mathrm{A})$ upto $100 \mathrm{MHz}$ frequency range

source is functional if the frequency of operation is below $100 \mathrm{MHz}$.

Another important aspect of a current source is its output impedance. Simply, output impedance is the impedance seen by the load at point of its connection. To simulate output impedance of a circuit at the output node one would replace the load with a voltage source, perform transient simulation and determine the amount of current entering or leaving the node for a change in voltage of the voltage source.

Mathematically, if $\Delta I_{o}$ is the change in current at the output node when the voltage at the output node changes by $\Delta V_{o}$.

$$
Z_{\text {OUT }}=\frac{\Delta I_{o}}{\Delta V_{o}}
$$


The output impedance simulation performed on basic Howland current source results in current at the output as shown in the figure 3.28.
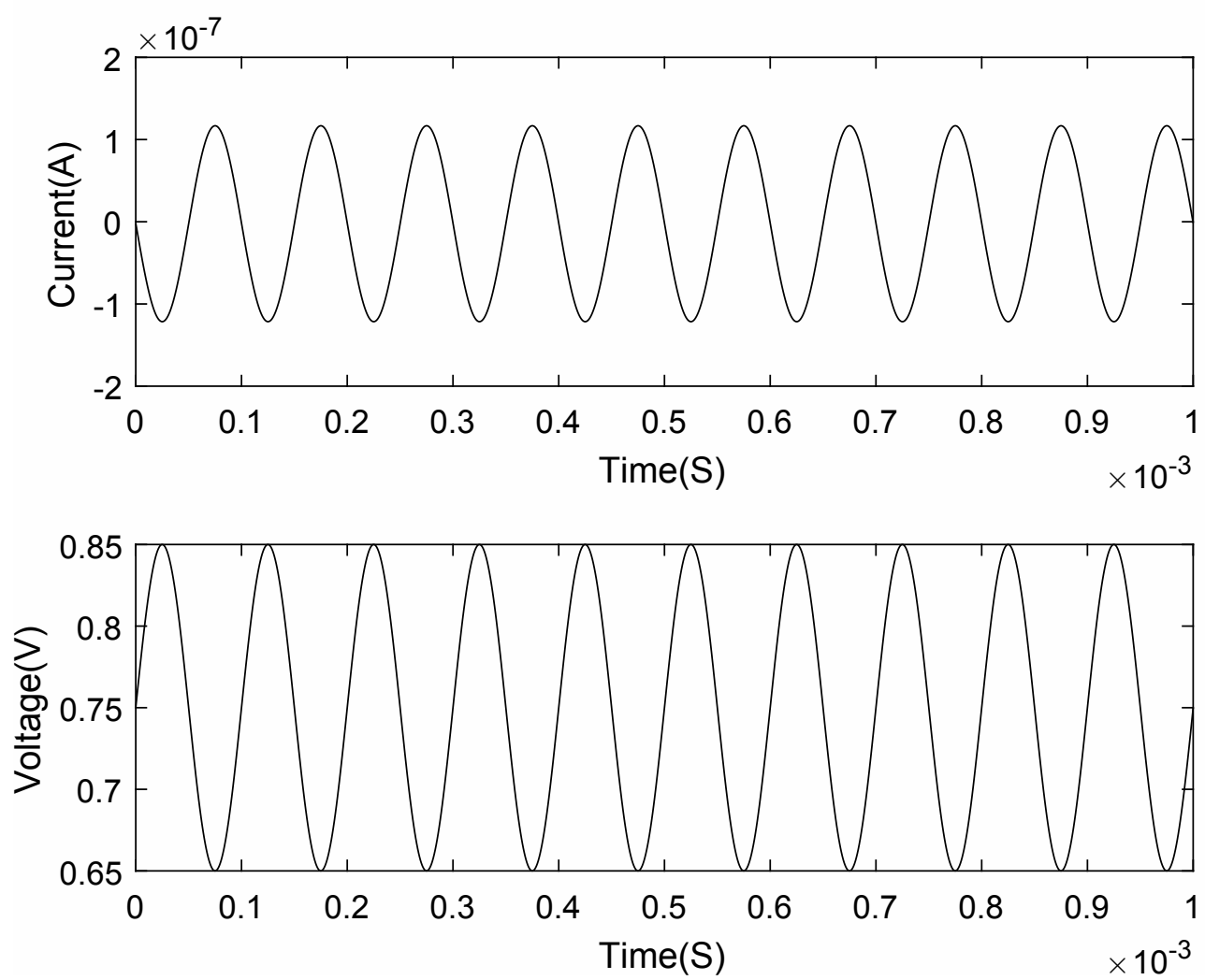

Figure 3.28: From top to bottom: Current output observed at the output node, voltage input signal. The voltage input of $200 \mathrm{mV}$ peak to peak voltage at $750 \mathrm{mV}$ DC bias is applied by replacing the load with a voltage source. The current output is observed at the node of the voltage source and observed current has a peak-to-peak magnitude of 945pA.

From the illustrated plot in the figure 3.28 , if $\Delta V_{o}$ is $200 \mathrm{mV}$, the current output, $\Delta I$, is $945 \mathrm{pA}$. Therefore, the output impedance is $211.6 \mathrm{M} \Omega$.

$$
Z_{O U T}=\frac{200 m V}{945 p A}=211.6 M \Omega
$$

The calculated output impedance is a very high value that is obtained only when the resistors used for simulation are perfectly matched. This is seldom the case. Process and temperature variations introduces mismatches in the circuits and its 
components all the time. Hence, a realistic approach towards output impedance is introducing a mismatch in the key components of the circuit and determining its value.

In the basic Howland current source, changing the input and feedback resistors by $1 \%$ the change in the output current is shown in the plot 3.19 .

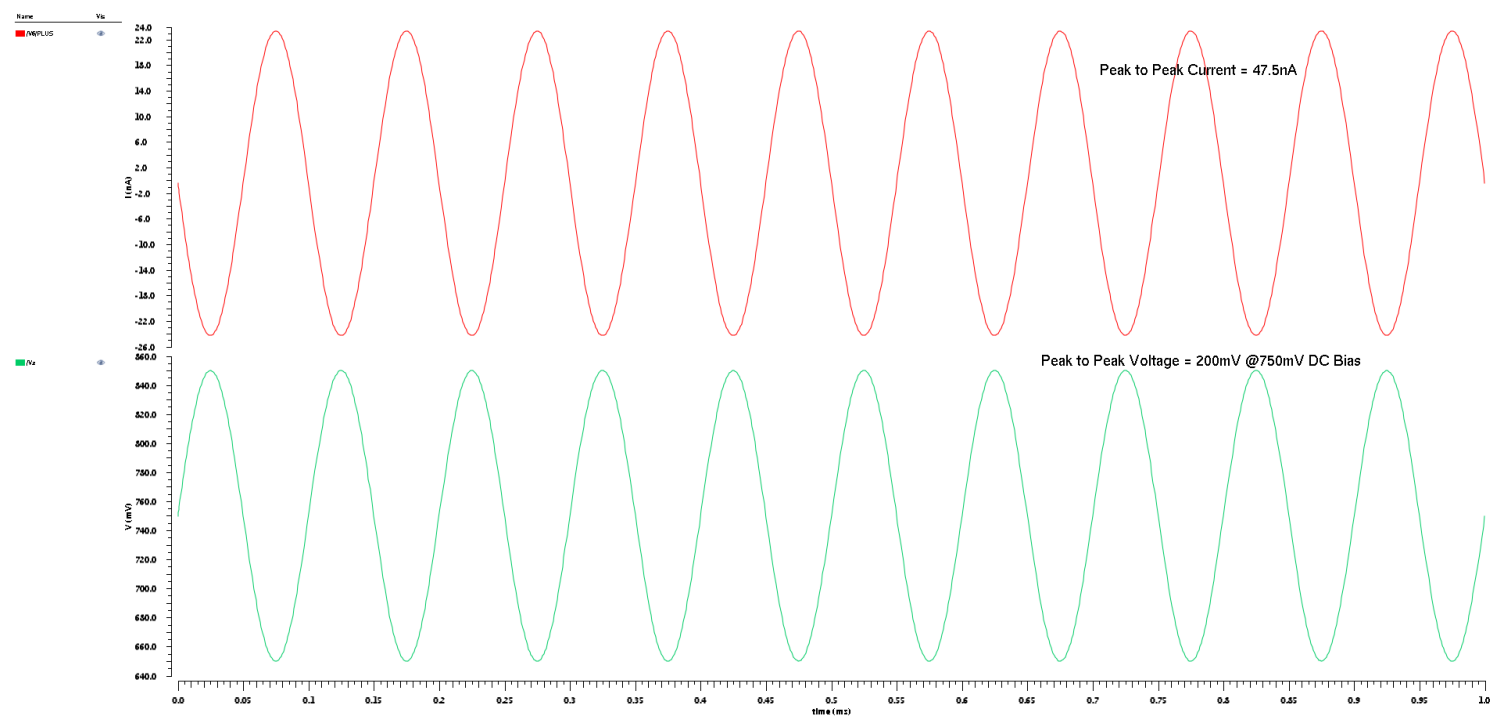

Figure 3.29: From top to bottom: Current output observed at the output node, voltage input signal. The voltage input of $200 \mathrm{mV}$ peak to peak voltage at $750 \mathrm{mV}$ DC bias is applied by replacing the load with a voltage source. The current output is observed at the node of the voltage source and observed current has a peak-to-peak magnitude of 47.5nA. 
Calculating the output impedance using the equation

$$
Z_{\text {OUT }}=\frac{200 \mathrm{mV}}{47.5 n}=4.2 \mathrm{M} \Omega
$$

A $1 \%$ mismatch in resistors resulted in an output impedance of $4.2 \mathrm{M} \Omega$.

The preceding simulations and discussion demonstrated how the output current is dependent upon the input resistor, $R_{1}$. Transient simulation outputs in the figure 3.25 distinctly shows that the peak current in case of a $100 \mathrm{~K} \Omega$ input resistor is $\sim 1 \mu \mathrm{A}$. The magnitude of this current is too low. To increase the current either the differential input has to be increased or the input resistor resistance has to be decreased.

The following figure shows the transient simulation current output for a reduced input, $R_{1}$, and feedback resistor, $R_{2}$. The peak-to-peak voltage input applied to the current source is $200 \mathrm{mV}$ at a common mode DC bias of $750 \mathrm{mV} . R_{1}$ and $R_{2}$ are reduced to $\sim 20 \mathrm{~K} \Omega$. 


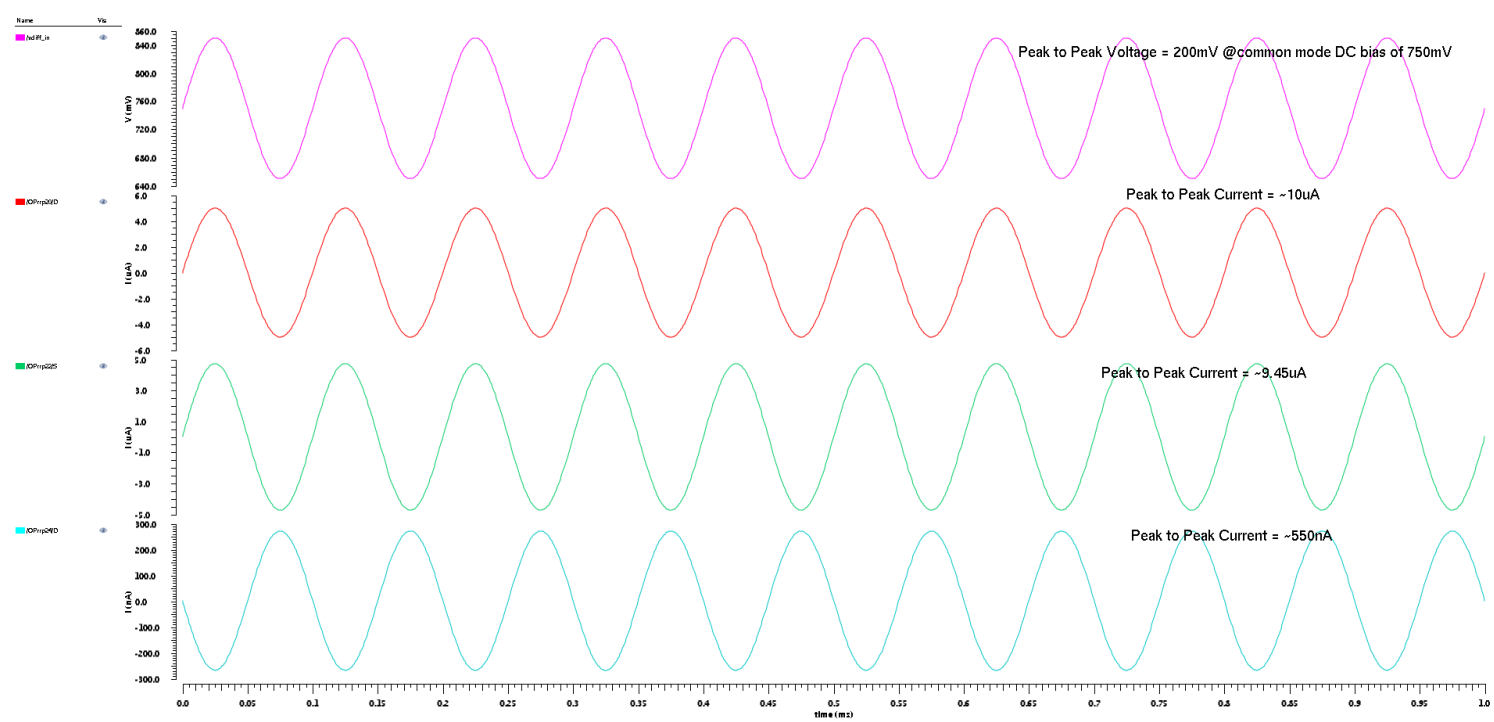

Figure 3.30: From top to bottom: $200 \mathrm{mV}$ peak-to-peak differential voltage input signal at a common mode DC of $750 \mathrm{mV}$ and frequency $10 \mathrm{kHz}$, load current with a peak-to-peak value $\sim$ $10 \mu \mathrm{A}$, Current in the input resistor with a peak-to-peak value $\sim 9.45 \mu \mathrm{A}$, current in the feedback resistor with a peak-to-peak value $\sim 550 \mathrm{nA}$. The current in the input and the feedback resistor add up to produce the load current. 
The figure 3.30 shows a current output with an amplitude of $5 \mu \mathrm{A}$. When the input resistance, $R_{1}$, is reduced to a fifth of its initial value, the current has increased factor of five. This not only validates the guiding equation, equation 2.21 , for the output current of the current source, on the hindsight it demonstrates the limitation of the current source.

The inability of the basic Howland current source to generate large currents stem from

a.) Large current can be generated if the differential input amplitude is increased. The increase in differential voltage input cannot be indefinite not only because of the ICMR range of the op-amp but also because a large signal might change the bias of the differential input transistors in the op-amp. Change in operating point of the input transistor could result in reduced operational gain which is undesired of an op-amp in basic Howland current source.

b.) Reducing the resistance of the resistors connected to the input terminals of the op-amp could yield higher currents. Not only does the output impedance see a drastic reduction in its value, the input impedance of the op-amp reduces significantly. Any input impedance mismatch regardless of the factor causing it coupled with already reduced impedance is a serious matter of concern because the CMRR of the op-amp goes considerably making the circuit vulnerable to noise and other issues.

\subsubsection{Modified Howland Current Source}

A modified Howland current source, aka improved Howland current source, is variant of Howland current source in which one of feedback resistors is split into 
two components and load resistor is connected as shown in the figure 3.31.

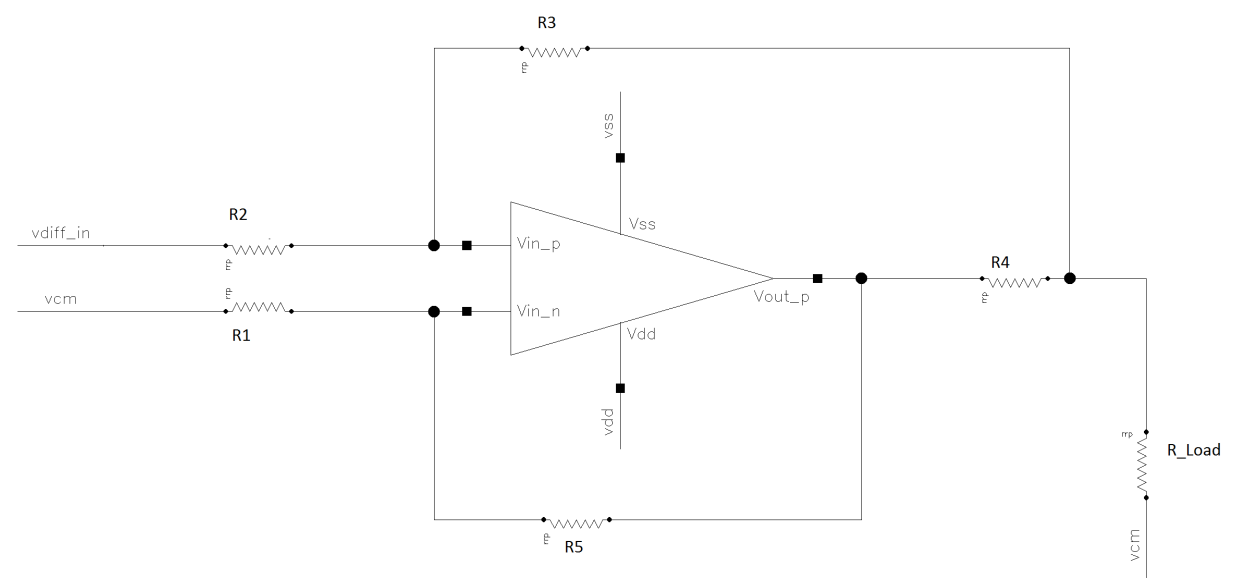

Figure 3.31: The figure illustrates the modified Howland current source circuit. The noticeable differences when compared to the schematic of basic Howland current source is the positive feedback resistor divided into R3 and R4, and the load resistor connected to the R4 and R3 connection node.

The modified Howland current source seen in the figure 3.31, as illustrated is an opamp based voltage controlled current source with positive and negative feedback. The positive and negative negative feedback paths are balanced when the resistor ratio condition shows in equation 3.23 is met.

$$
\frac{R_{1}}{R_{5}}=\frac{R_{2}}{R_{3}+R_{4}}
$$

As discussed in Chapter 2, section 2.4.1, the output current of a modified Howland current source is a function of the resistor $R_{4}$ in the schematic 3.21 and the differential input voltage. The output current is given by

$$
I_{\text {OUT }}=\frac{V_{\text {diff }}}{R_{4}}
$$

The following transient simulation plots validate the above equation and working 
of the current source in question.
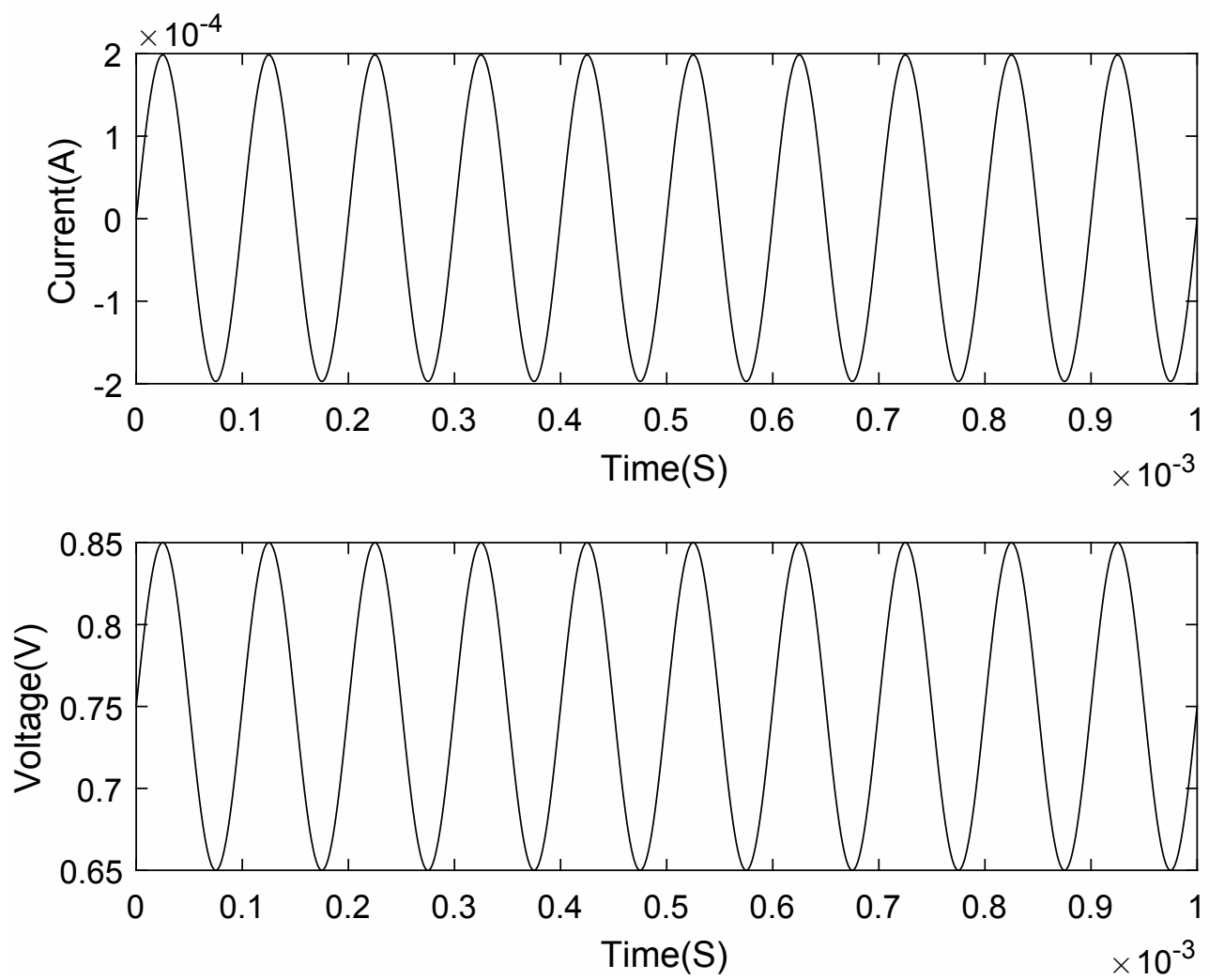

Figure 3.32: From top to bottom: $200 \mathrm{mV}$ peak-to-peak differential voltage input signal at a common mode DC of $750 \mathrm{mV}$ and frequency $10 \mathrm{kHz}$, load current with a peak-to-peak value $\sim 195.7 \mu \mathrm{A}$, Current in the $R_{4}$ resistor with a peak-to-peak value $\sim 195.8 \mu \mathrm{A}$, current in the feedback resistor with a peak-to-peak value $\sim 2 \mu \mathrm{A}$.

As seen in the figure 3.32 , the current in the resistor $R_{4}$ is more or less equal to the current in the load resistor. This is because, once the positive and negative feedback paths are balanced the excess current due to differential input is released by the current source through $R_{4}$ so that the balance is not disturbed. Therefore, the feedback current is due to the common mode at the input front end and the current in the load is due to differential input applied to the input.

The load current in the plot shown in figure 3.32 can be varied by varying the input voltage. For an $R_{4}$ of $1 \mathrm{~K} \Omega$ and a load of $1 \mathrm{~K} \Omega$, the load current observed 
for an input of $200 \mathrm{mV}$ peak-to-peak differential input is $\sim 195 \mu \mathrm{A}$, and the output current is $\sim 98 \mu \mathrm{A}$ for a differential input of $100 \mathrm{mV}$ peak-to-peak voltage. The power rails are $1.5 \mathrm{~V}$ and $0 \mathrm{~V}$. The frequency of the input is $10 \mathrm{kHz}$.
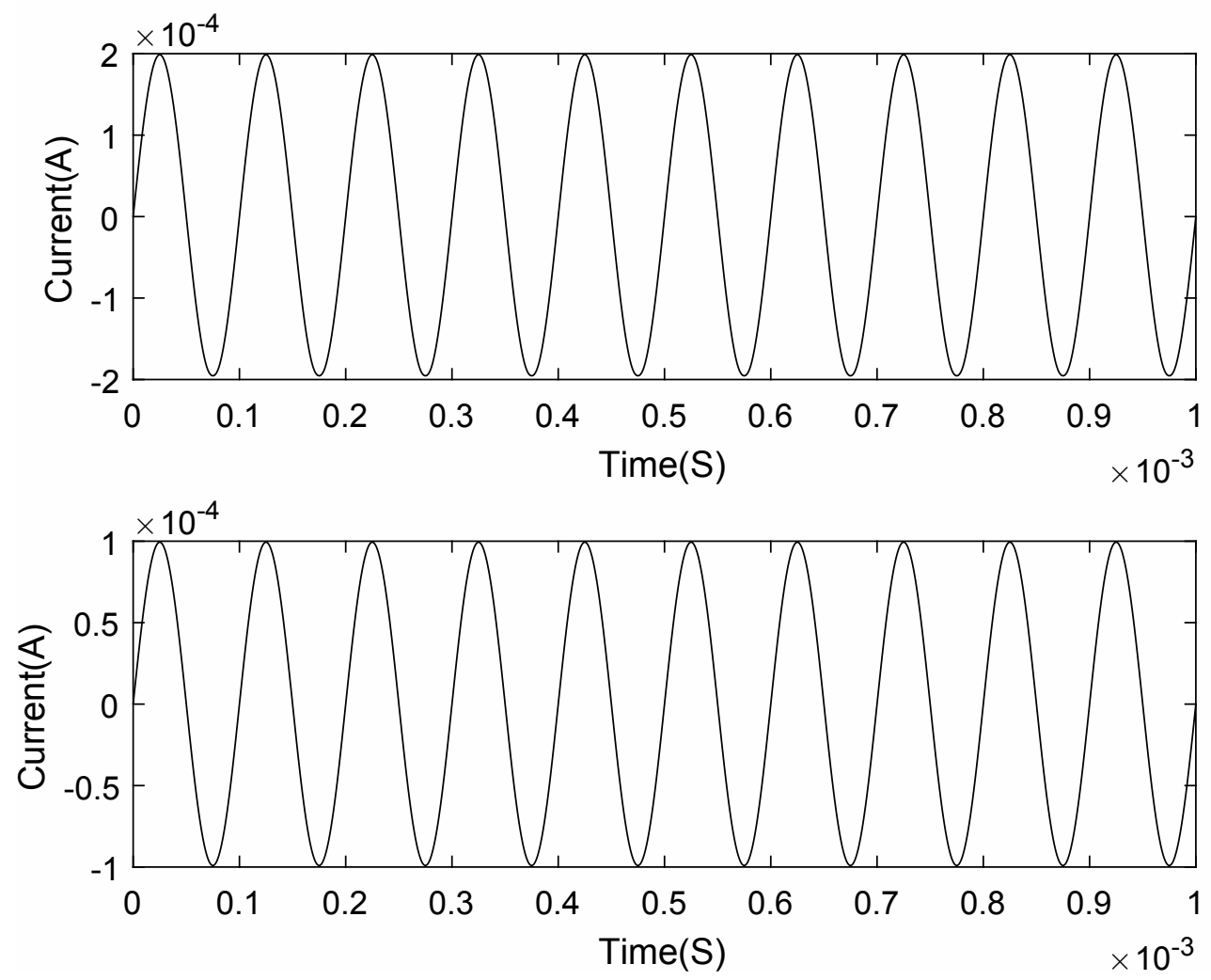

Figure 3.33: The figure shows current outputs for $200 \mathrm{mV}$ differential input and $100 \mathrm{mV}$ differential input of frequency $10 \mathrm{kHz}$ in both the cases. The output current when the input is $200 \mathrm{~m} V_{p p}$ is $\sim 195 \mu \mathrm{A}$ peak-to-peak and is shown in blue. The output current when the input is $100 \mathrm{~m} V_{p p}$ is $\sim 100 \mu \mathrm{A}$ peak-to-peak and is shown in red.

The output current can also be changed by changing the value of $R_{4}$. If $R_{4}$ is $500 \Omega$, for a differential voltage input of $100 \mathrm{mV}$ at a common mode DC bias of $750 \mathrm{mV}$ and frequency of $10 \mathrm{kHz}$ is the output current is $\sim 200 \mu \mathrm{A}$. 


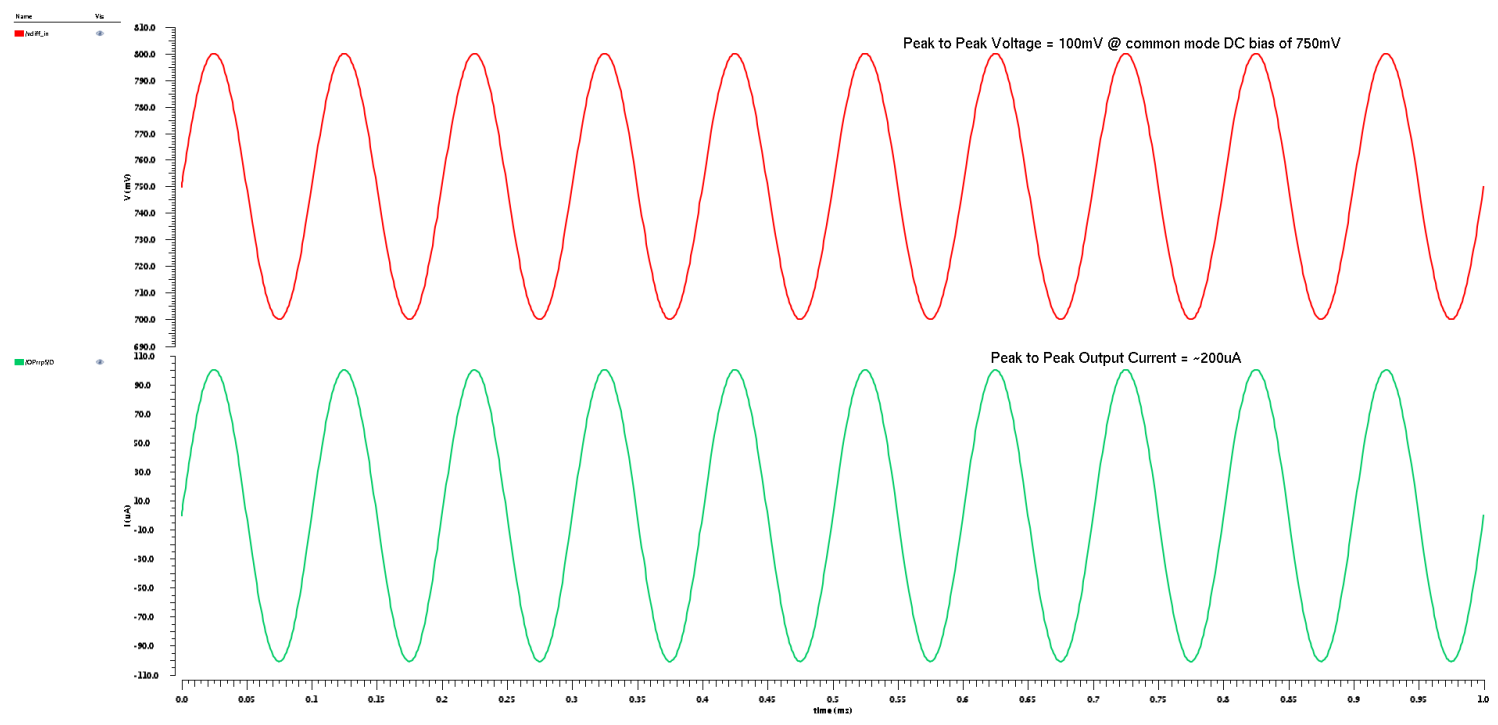

Figure 3.34: From top to bottom: $200 \mathrm{mV}$ peak-to-peak differential voltage input signal at a common mode $\mathrm{DC}$ of $750 \mathrm{mV}$ and frequency $10 \mathrm{kHz}$, load current with a peak-to-peak value $\sim 200 \mu A$. 
The output impedance of a modified Howland current source is given by the following equation [13]

$$
Z_{\text {OUT }}=\frac{\left(R_{2}+R_{5}\right) \| R_{4}}{1-\beta_{f b}} \frac{(1-T)^{2}}{4 T}
$$

where $\mathrm{T}$ is tolerance of the resistors and $\beta_{f b}$ is

$$
\beta_{f b}=\frac{R_{1}}{R_{1}+R_{5}}
$$

Substituting the values in the equations 3.25 and 3.26 gives $Z_{O U T}$ and assuming $\mathrm{T}$ is $1 \%$

$$
Z_{O U T} \sim 49 K \Omega
$$

The equations 3.25 and 3.26 show the dependency of the output impedance on the values of branch resistances and the gain setting resistor, $R_{4}$. This shows that output impedance, $Z_{O U T}$, also increases with an increase in the gain setting resistor resistance. But, increasing the gain setting resistance reduces transconductance gain of the current source and reduces the output current. Also, increasing the value of $R_{4}$ increases the chances of disturbing the balance in the feedback paths. Though the modified Howland current source is an improvement upon basic Howland current source with regards to the transconductance gain, the output impedance is low.

Compliance of a current source is the voltage margin of the load voltage within which the circuit acts as a current source. When compared to basic Howland current source, modified Howland current source offers increased compliance to the load, however the increase in compliance of the modified Howland current source 
is not sufficient and it is illustrated in the following plots.

For a load of $1 \mathrm{~K} \Omega$ and varying differential input, varying from $50 \mathrm{mV}$ to $250 \mathrm{mV}$ with a step size of $50 \mathrm{mV}$, at a common mode DC bias of $750 \mathrm{mV}$ at a frequency of $10 \mathrm{kHz}$, the output current is as seen in the figure 3.35 .

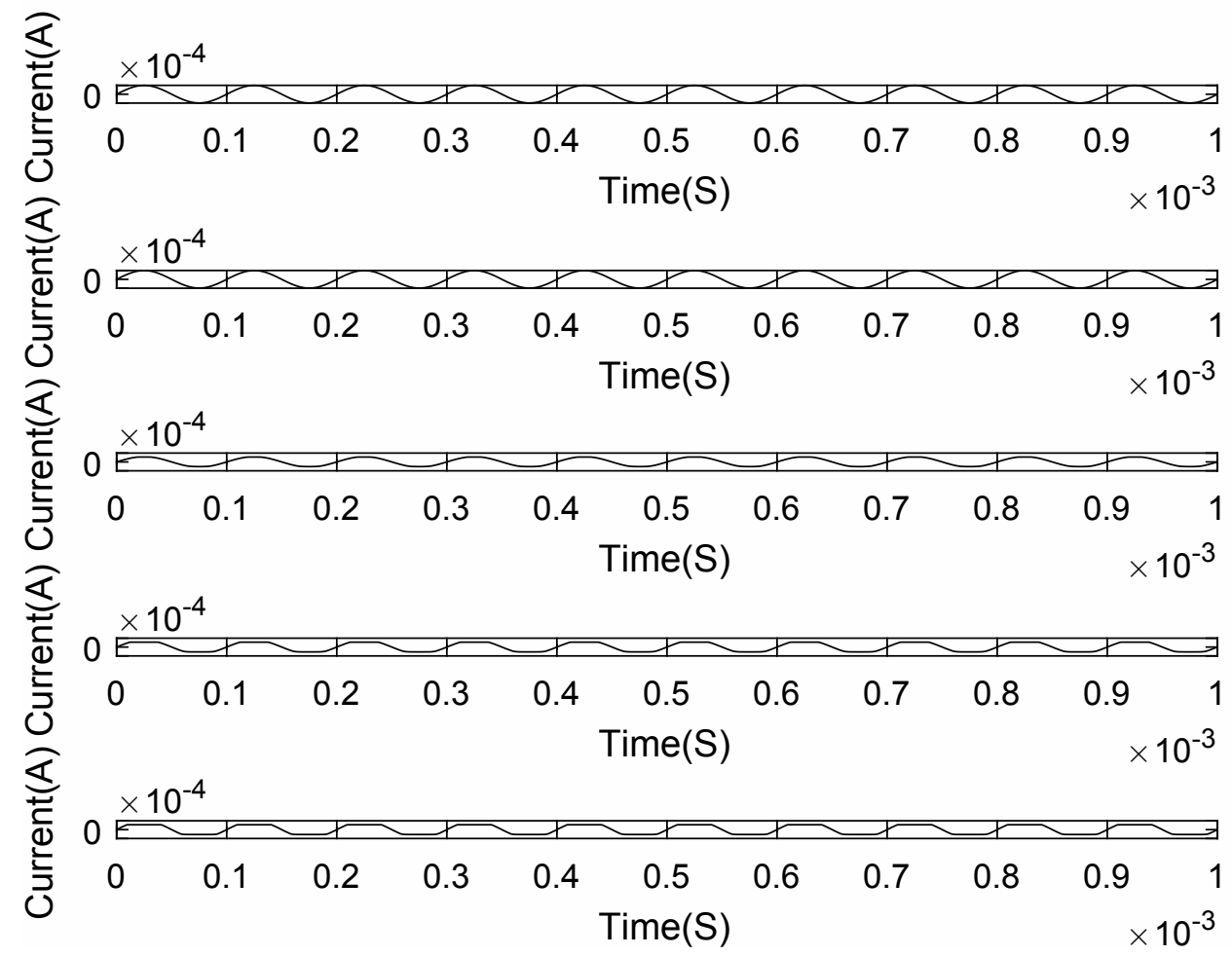

Figure 3.35: From top to bottom: Peak-to-peak output current of $\sim 98.5 \mu \mathrm{A}$ for input of $50 \mathrm{mV}$ peak voltage, Peak-to-peak output current of $\sim 198 \mu \mathrm{A}$ for input of $100 \mathrm{mV}$ peak voltage, Peak-topeak output current of $\sim 249.7 \mu \mathrm{A}$ for input of $150 \mathrm{mV}$ peak voltage, Peak-to-peak output current of $\sim 251.1 \mu \mathrm{A}$ for input of $200 \mathrm{mV}$ peak voltage, Peak-to-peak output current of $\sim 250.5 \mu \mathrm{A}$ for input of $250 \mathrm{mV}$ peak voltage 
Seen in the figure 3.35, the output current for a differential input of $300 \mathrm{mV}$ peak-to-peak voltage and onwards clips indicating limited compliance of the modified Howland current source circuit.

The upper limit on the output voltage swing of the op-amp along with the imbalance in the feedback paths of the circuit causes the output voltage of the op-amp saturate for higher input voltages. The saturated output voltage limit is roughly the limited compliance of the output current of the current source. This is visible in the following plots.

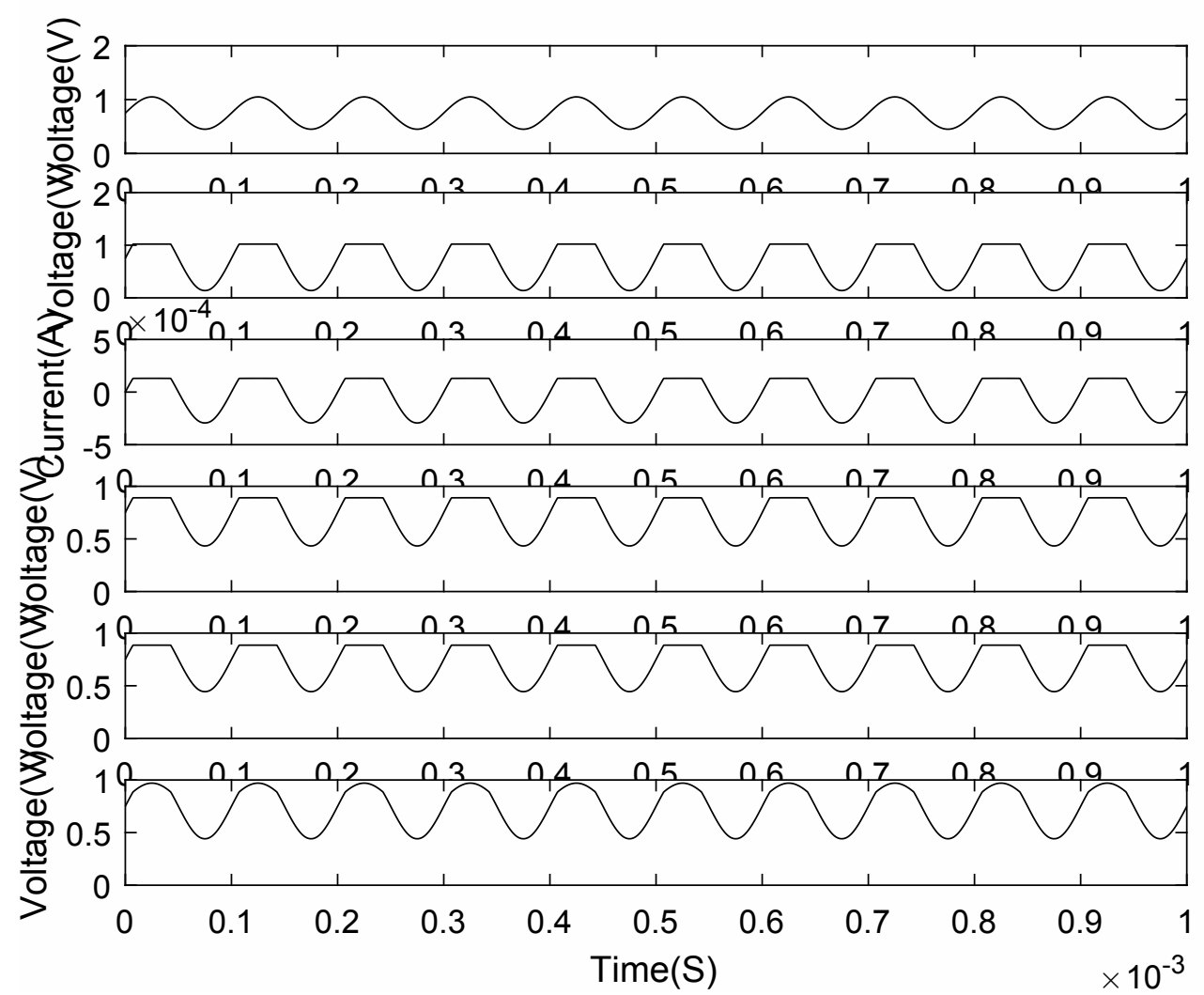

Figure 3.36: From top to bottom: Differential input voltage of magnitude $600 \mathrm{mV}$ at $750 \mathrm{mV}$ common mode DC bias, output voltage of the op-amp with maximum peak voltage, load current plot, load voltage, inverting voltage of the op-amp, non-inverting voltage of the op-amp 
The inputs and outputs illustrated in the figure 3.36 are the plots of the transient analysis performed by subjecting the modified Howland current source to a peak-to-peak differential input of $600 \mathrm{mV}$ at a common mode $\mathrm{DC}$ of $750 \mathrm{mV}$. The power rails of the op-amp are $1.5 \mathrm{~V}$ and $0 \mathrm{~V}$. The minimum peak voltage of the op-amp output is $139 \mathrm{mV}$ (the difference between lowest power supply and $139 \mathrm{mV}$ is $139 \mathrm{mV}$ ), where as the maximum voltage is $1.02 \mathrm{~V}$ (the difference between $1.5 \mathrm{~V}$ and $1.02 \mathrm{~V}$ is $\sim 500 \mathrm{mV}$ ). Moreover, because the lower limit of the output voltage swing is close to $0 \mathrm{~V}$ the current output of the circuit follows the principle equation, equation 3.24, of the modified Howland current source for the negative voltage peak of the input. This confirms that the limited output swing indicates limited compliance of the modified Howland current source. 


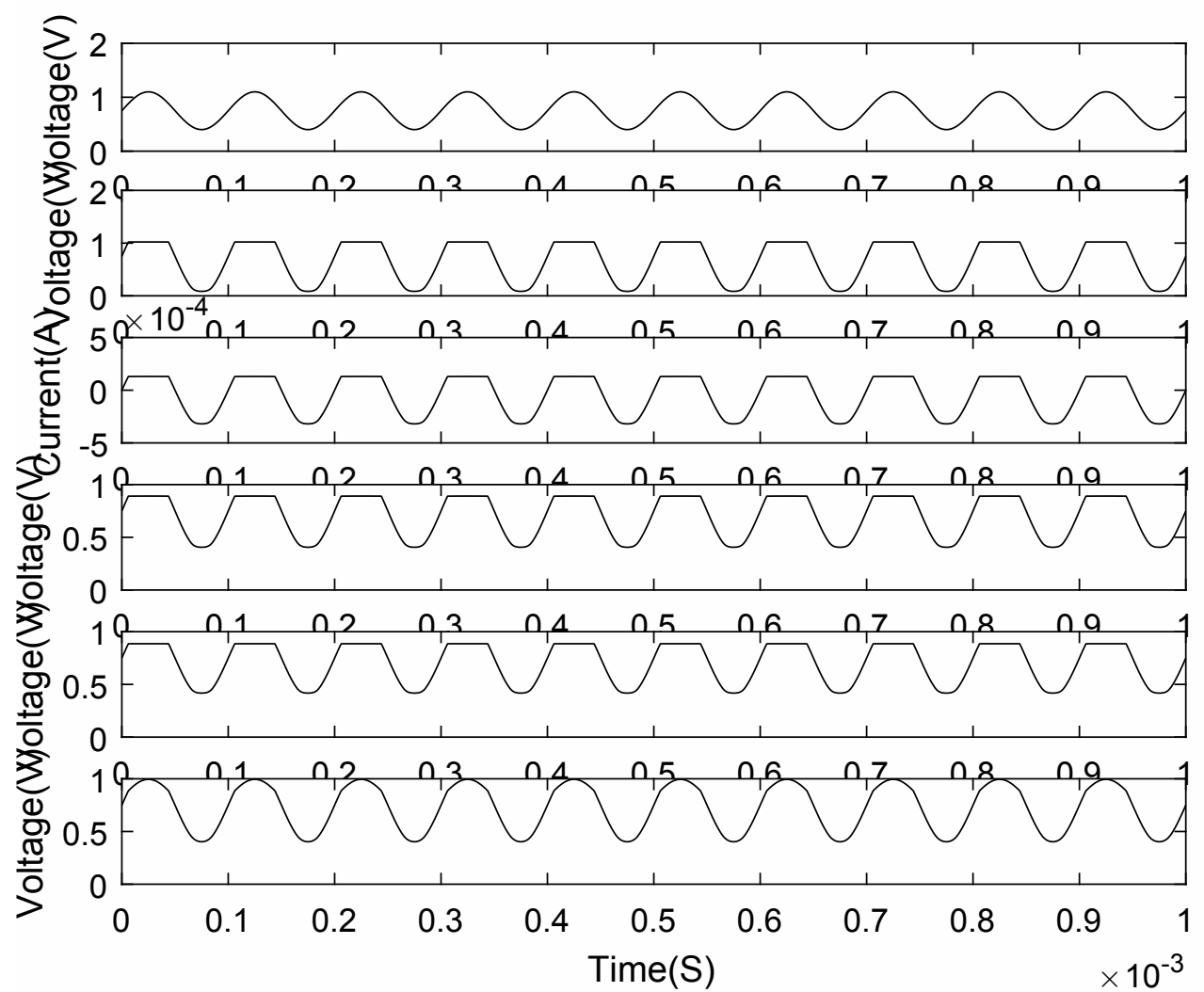

Figure 3.37: From top to bottom: Input voltage of magnitude $700 \mathrm{mV}$ at $750 \mathrm{mV}$ common mode DC bias, output voltage of the op-amp with maximum peak voltage, load current plot, load voltage, inverting voltage of the op-amp, non-inverting voltage of the op-amp 
In the transient simulation plots in the figure 3.37, the non-linearity is seen clearly in the output current plot.

For a differential input of $700 \mathrm{mV}$ peak-to-peak voltage, going by the equation 3.24 for the modified Howland current source, the expected output current is $\sim 350 \mu \mathrm{A}$ if the gain setting resistor is $1 \mathrm{~K} \Omega$.

$$
I_{O U T}=\frac{350 m V}{1 K \Omega}
$$

But the output current observed is $\sim 318.5 \mu \mathrm{A}$.

Also, observing the inverting and non-inverting voltages, it is clear that the imbalance in the feedback paths reduce the output voltage swing and causes the voltages and currents to saturate.

The low output impedance, insufficient compliance and lack of support for floating load prompts the usage of mirrored modified Howland current source.

\subsubsection{Mirrored Modified Howland Current Source}

Reiterating on the drawbacks of the modified Howland current source, it suffers from

a.) Limited compliance of the circuit. Though the modified Howland current source can produce currents larger than basic Howland current source, the output current saturates for larger currents outputs that are needed for bioimpedance measurements.

b.) A modified Howland current source with a dual supply op-amp supports loads that are grounded. The simulation schematics of the current sources illustrate that load resistance is biased at mid-rail voltage. For a basic Howland current source 
with a single supply op-amp, if the load resistor is connected to $0 \mathrm{~V}$ the common mode at the input of the op-amp is pulled down to $0 \mathrm{~V}$. A $0 \mathrm{~V}$ common mode input to the op-amp tampers the bias of the differential pair input transistors reducing the gain and CMRR of the op-amp. In the case of modified Howland current source, connecting the load resistor to $0 \mathrm{~V}$ induces imbalance in the feedback path and leads to distortion in the output current.

c.) Low ouptut impedance.

A mirrored modified Howland current source shown in figure 3.38 contains a modified Howland current source sourcing current into the load resistor and another modified Howland current source sinking the same amount of current from the load resistor. 


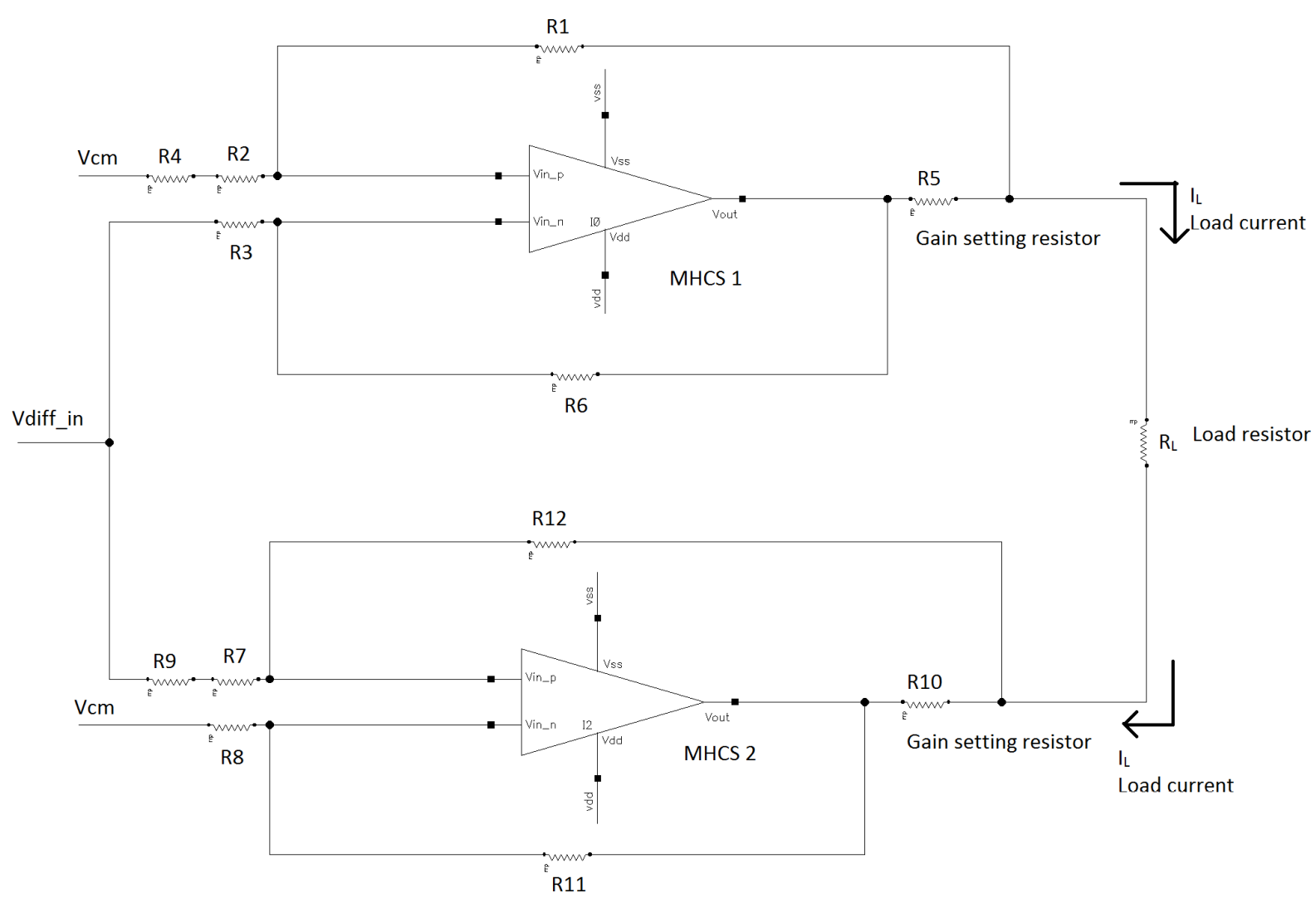

Figure 3.38: The figure shows the designed mirrored modified Howland current source. The load resistor, $R_{L}$, is a floating load and the current in the load is indicated by $I_{L}$. The resistor $R_{5}$ and the $R_{10}$ are the gain setting resistors of the individual modified Howland current sources upon which the load current in the load is mainly dependent on. 
In the figure 3.38, MHCS 1 and MHCS 2 are modified Howland current sources which source and sink current in the load resistor respectively. The resistors $R_{5}$ and $R_{10}$ are gain setting resistors which set the transconductance gain of the individual Modified Howland current source. $R_{1}, R_{6}, R_{11}$ and $R_{12}$ are feedback resistors of the modified Howland current sources, and the rest of the resistors are input resistors.

The resistor relationship equations of the resistors in the mirrored modified Howland current source are

$$
\begin{gathered}
R_{1}=R_{6}=R_{3}=R_{2}=R \\
R_{12}=R_{11}=R_{8}=R_{7}=R \\
R_{4}=R_{5}=r \\
R_{10}=R_{9}=r
\end{gathered}
$$

The resistor relationships result in

$$
\begin{aligned}
\frac{R_{6}}{R_{3}} & =\frac{R_{5}+R_{1}}{R_{2}+R_{4}} \\
\frac{R_{11}}{R_{8}} & =\frac{R_{10}+R_{12}}{R_{7}+R_{8}}
\end{aligned}
$$

When gain setting resistors are equal, the output currents of both the modified Howland current sources is equal. However, since the differential input is applied to non-inverting input in one modified Howland current source and inverting input in the other current source the current is 'mirrored' in the load.

If the resistances of the resistors are set as shown in the equations 3.35 and 3.36, the load current in the load resistor is $100 \mu \mathrm{A}$. For an input of $200 \mathrm{mV}$ peak-to-peak 
voltage at a common mode DC bias of $750 \mathrm{mV}$ and frequency of $10 \mathrm{kHz}$, the load current in the load is as shown in the figure 3.39 .

$$
\begin{gathered}
R=100 K \Omega \\
r=1 K \Omega
\end{gathered}
$$

The load current is a function, given by the equation 3.29 and illustrated in the
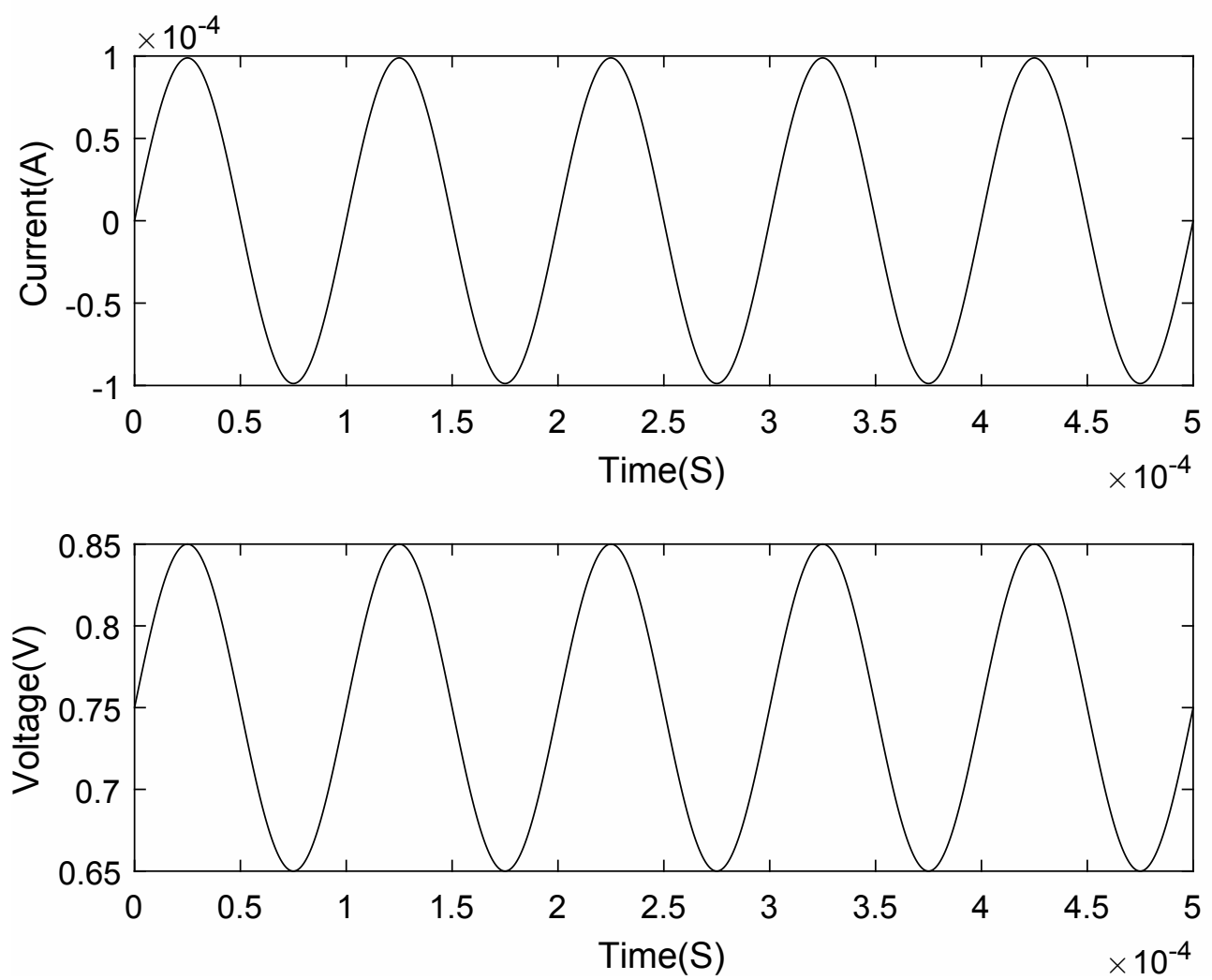

Figure 3.39: From top to bottom: A $200 \mathrm{mV}$ differential input peak-to-peak voltage at a common mode DC bias of $750 \mathrm{mV}$, current in the load resistor with a peak current of $\sim 100 \mu \mathrm{A}$. Since the gain setting resistor is $1 \mathrm{~K} \Omega$, for a peak voltage of $100 \mathrm{mV}$ the load current is $100 \mu \mathrm{A}$.

figure 3.39, of the input voltage and the gain setting resistors, $R_{5}$ and $R_{10}$.

$$
I_{L}=\frac{V \operatorname{diff\_ in}}{r}
$$

Hence, the load current can be increased of decreased by varying the differential input, Vdiff_in and/ or resistance of the gain setting resistor, $r$. 
If the differential input voltage varies from $50 \mathrm{mV}$ to $200 \mathrm{mV}$ with a common mode DC bias of $750 \mathrm{mV}$, the output load current changes from $\sim 50 \mu \mathrm{A}$ to $\sim 200 \mu \mathrm{A}$ as shown in the fioure 240 If the resictane of oain settino recistor $r$ varied from
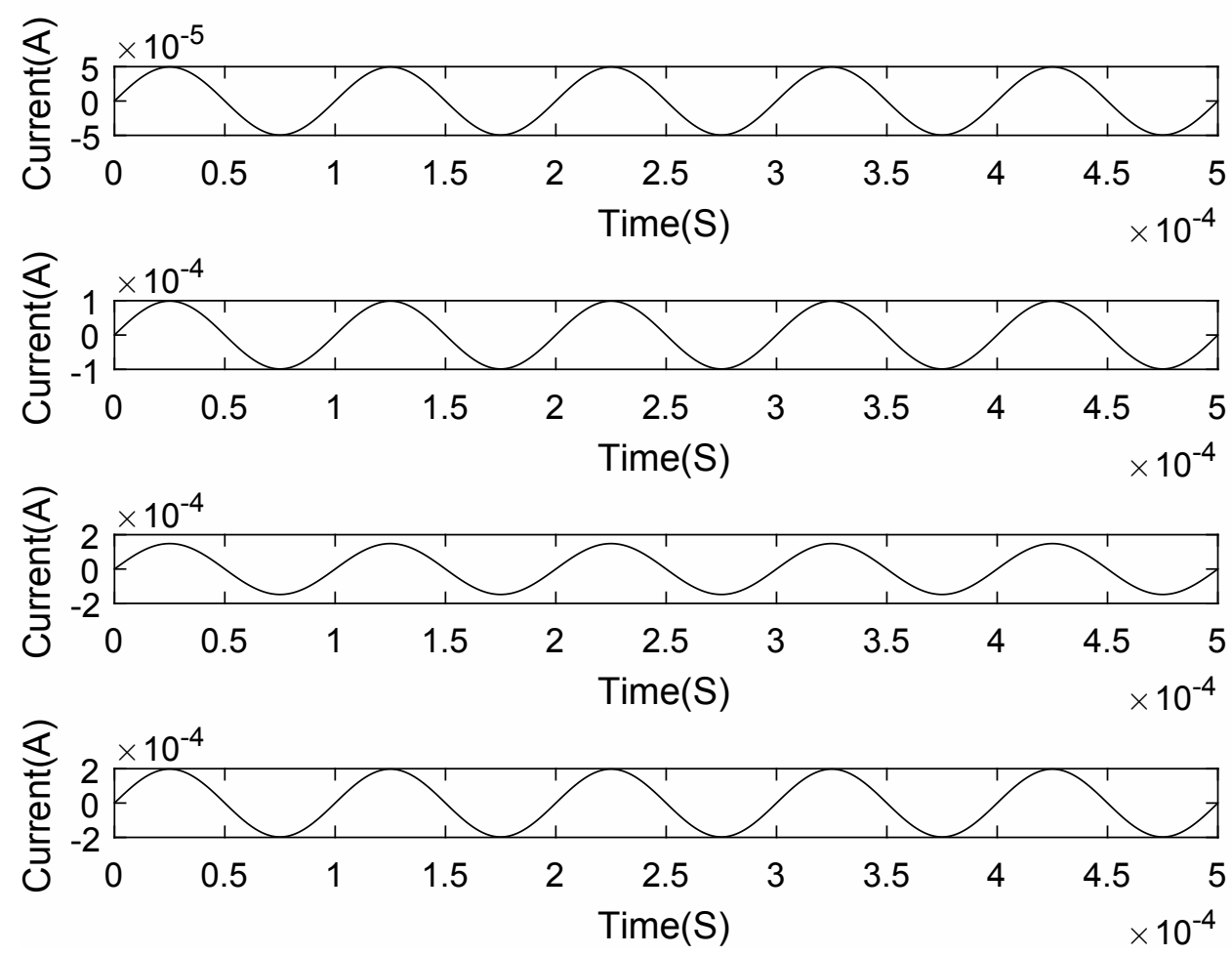

Figure 3.40: The figure shows peak load currents for differential input voltage signals with peak voltages $50 \mathrm{mV}$ to $200 \mathrm{mV}$ varying with a step size of $50 \mathrm{mV}$.

$750 \Omega$ to $2 \mathrm{~K} \Omega$ with a step size of $250 \Omega$ the varying load current plots are seen in the figure 3.40 . 


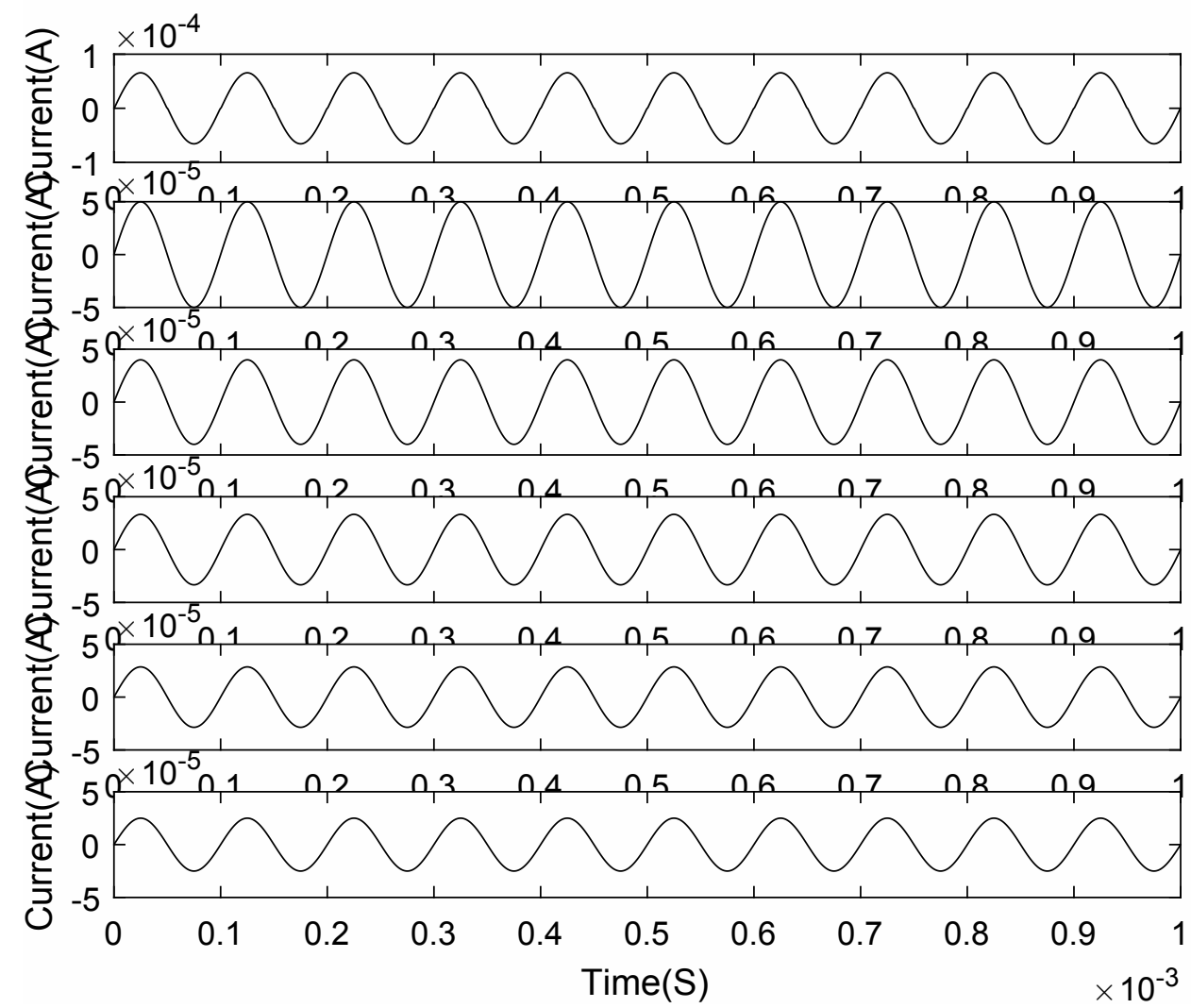

Figure 3.41: In the figure, load current plots are illustrated for varying resistance of the gain resistor. As expected, when the gain resistor value changes from $750 \Omega$ to $2 \mathrm{~K} \Omega$ the value of load current changes from $25 \mu \mathrm{A}$ to $65.6 \mu \mathrm{A}$. 


\section{Output Impedance}

A constant current regardless of the change in the value of the load resistance is a highly desired characteristic characteristic of a current source. The output current of a current source remains constant if the output impedance is very high.

The ratio of the change in output voltage to the change in output current at the output node is the output impedance of the circuit. The following simulation figure 3.42 illustrates the output impedance of the mirrored modified Howland current source. The output impedance is obtained by For a $1 \mathrm{mV}$ change in voltage at the
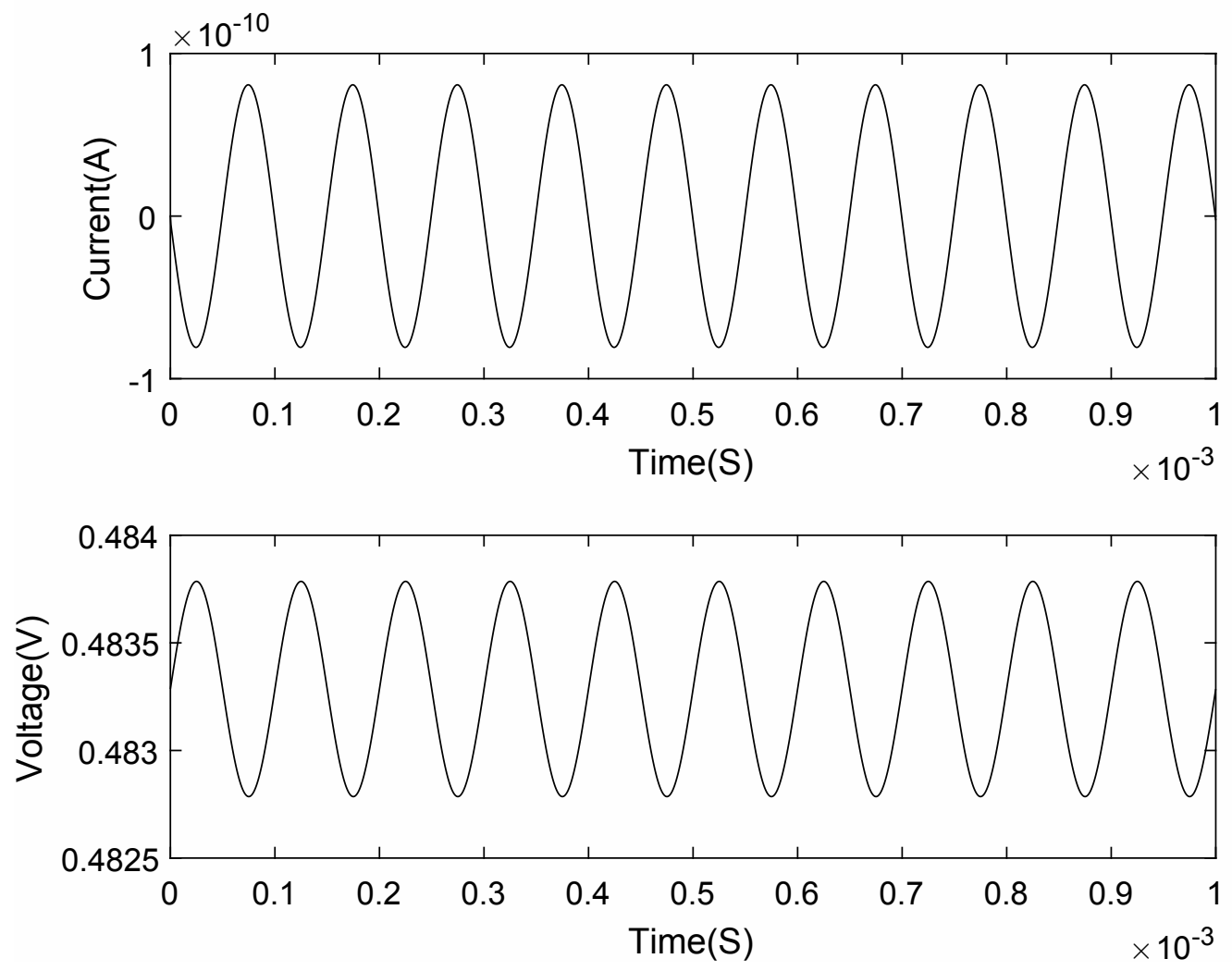

Figure 3.42: From top to bottom: Top signal is the input signal, $1 \mathrm{mV}$ peak-to-peak voltage, applied at the output node, bottom signal is the peak-to-peak output current, $161.2 \mathrm{pA}$, of the mirrored modified Howland current source.

output, the change in current is $161.2 \mathrm{pA}$. Thus, output impedance is

$$
Z_{O U T}=\frac{1 m V}{161.2 p A}=6.2 M \Omega
$$


The high output impedance of $6.2 M \Omega$ is a significant improvement upon modified Howland current source which has a much lower output impedance.

A current source with high output impedance is expected to emit the same amount of output current irrespective of the value of the load resistance. Regardless of the load resistance, for an input of $1 \mathrm{mV}$ peak voltage at a common mode DC bias of $750 \mathrm{mV}$ and frequency of $10 \mathrm{kHz}$, the expected current for a gain setting resistor of $1 \mathrm{~K} \Omega$ is $1 \mu \mathrm{A}$ peak current.
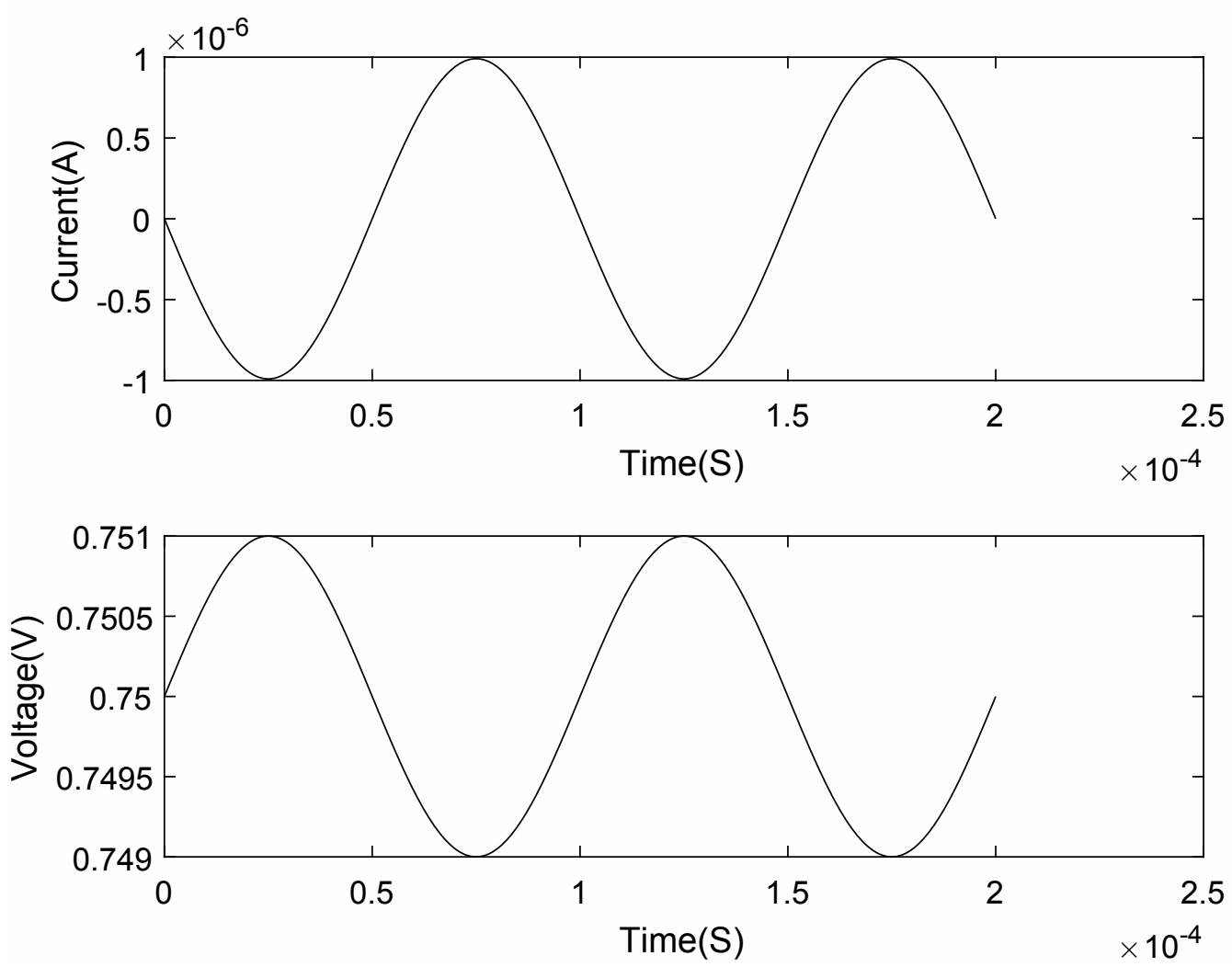

Figure 3.43: From top to bottom: Top signal is the input voltage signal of $1 \mathrm{mV}$ peak voltage, bottom signal represents the overlapping output currents of varying load resistance with a peak current magnitude of $\sim 1 \mu \mathrm{A}$

The overlapping output current plots evince the similar current outputs with same peak magnitude of $\sim 1 \mu \mathrm{A}$ for different load resistances.

\section{Compliance}


Compliance of an output current source is the voltage margin at the load beyond which the current output distorts and proper operation ceases.

In the case of modified Howland current source, the load biased at the mid-rail voltage limited the margin of the voltage output of the op-amp. Transient behavior of the current source displayed demonstrates the output current saturation due to limited compliance. Though made up of two modified Howland current sources, the mirrored modified Howland current source exhibits a wider compliance because the floating load is biased at a voltage much lower than the mid-rail voltage, meaning that the voltage margin of the individual op-amps has increased by that exact amount of voltage. This phenomenon can be observed in the following simulations.

For an input of $250 \mathrm{mV}$ peak voltage at a common mode DC bias of $750 \mathrm{mV}$ produces and output current of peak magnitude equal to $\sim 250 \mu \mathrm{A}$. In the figure 3.44 ,

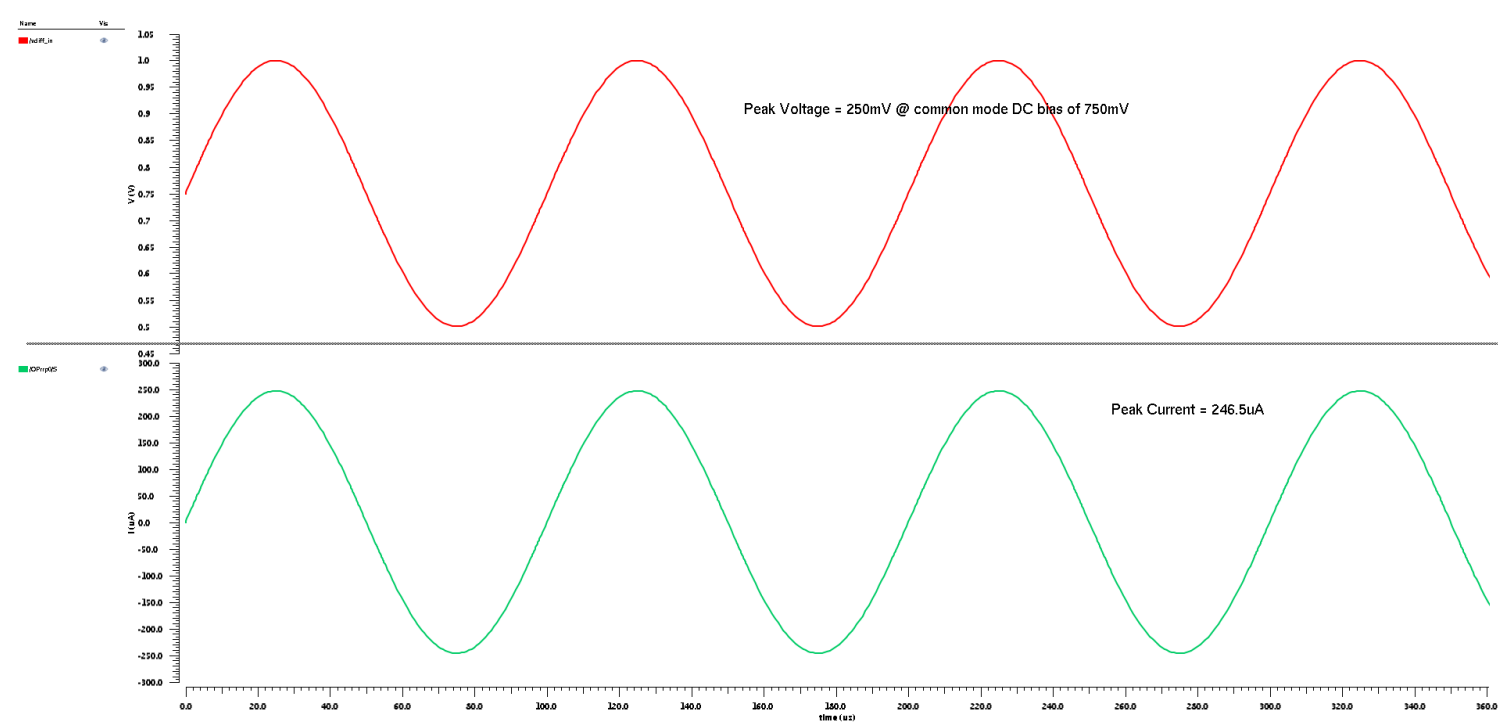

Figure 3.44: From top to bottom: Top signal is the input signal applied to the mirrored modified Howland current source of amplitude $250 \mathrm{mV}$ at a common mode DC bias of $750 \mathrm{mV}$, bottom signal is the output current of the circuit with a peak current of $246.5 \mu \mathrm{A}$ 
the peak output current is $246.5 \mu \mathrm{A}$, which is close to the expected output current value $\sim 250 \mu \mathrm{A}$. Contrary to the peak-to-peak output current of $\sim 500 \mu \mathrm{A}$ produced by the mirrored modifed Howland current source is the peak-to-peak output current of $\sim 250 \mu \mathrm{A}$ produced by the modified Howland current source for the same input. 


\section{Non-Linearity}

The current source employs op-amp which has a limited output swing, implying that the current source is operational within a limited input range, implying that the non-linearity of the current source be measured within the limited input range. Also, since the current source is operated in the low frequencies, i.e. below $100 \mathrm{kHz}$, total harmonic distortion is calculated as a measure for non-linearity.

For an input of peak amplitude $100 \mathrm{mV}$ at a common mode DC bias of $750 \mathrm{mV}$, the expected output current is $\sim 100 \mu \mathrm{A}$ for a gain setting resistance value of $1 \mathrm{~K} \Omega$. The Fourier spectrum of the output current seen in the figure 3.45 confirms the output current at the fundamental frequency, $10 \mathrm{kHz}$, is very close to the expected value and that is $98.8 \mu \mathrm{A}$.

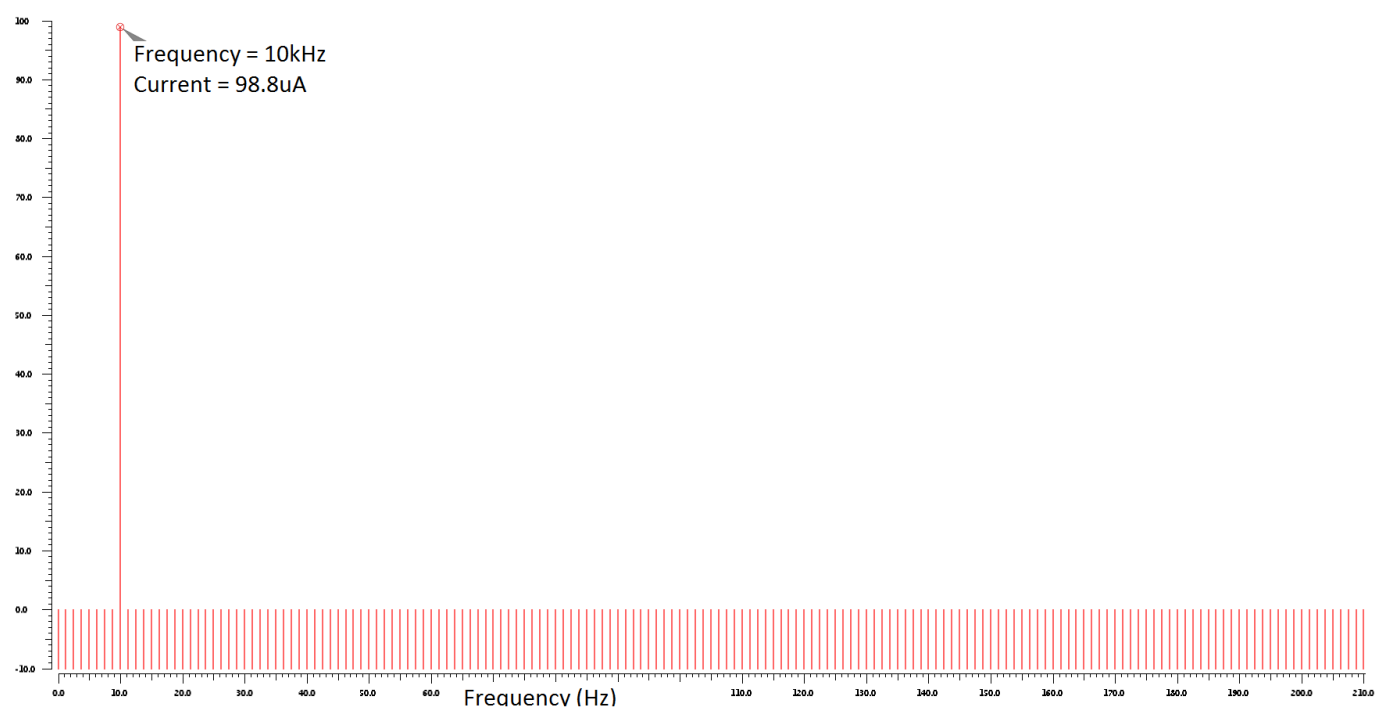

Figure 3.45: Discrete fourier transform (DFT) spectrum of load current at the output is illustrated in the figure. At input frequency of $10 \mathrm{kHz}$ and differential voltage input of $100 \mathrm{mV}$ peak voltage, the magnitude of the output current is $98.8 \mu \mathrm{A}(\sim 100 \mu \mathrm{A})$. 
Illustrated in the figure 3.46 is the magnitude of output current in the harmonics of the fundamental frequency. The magnitude of current in the harmonics

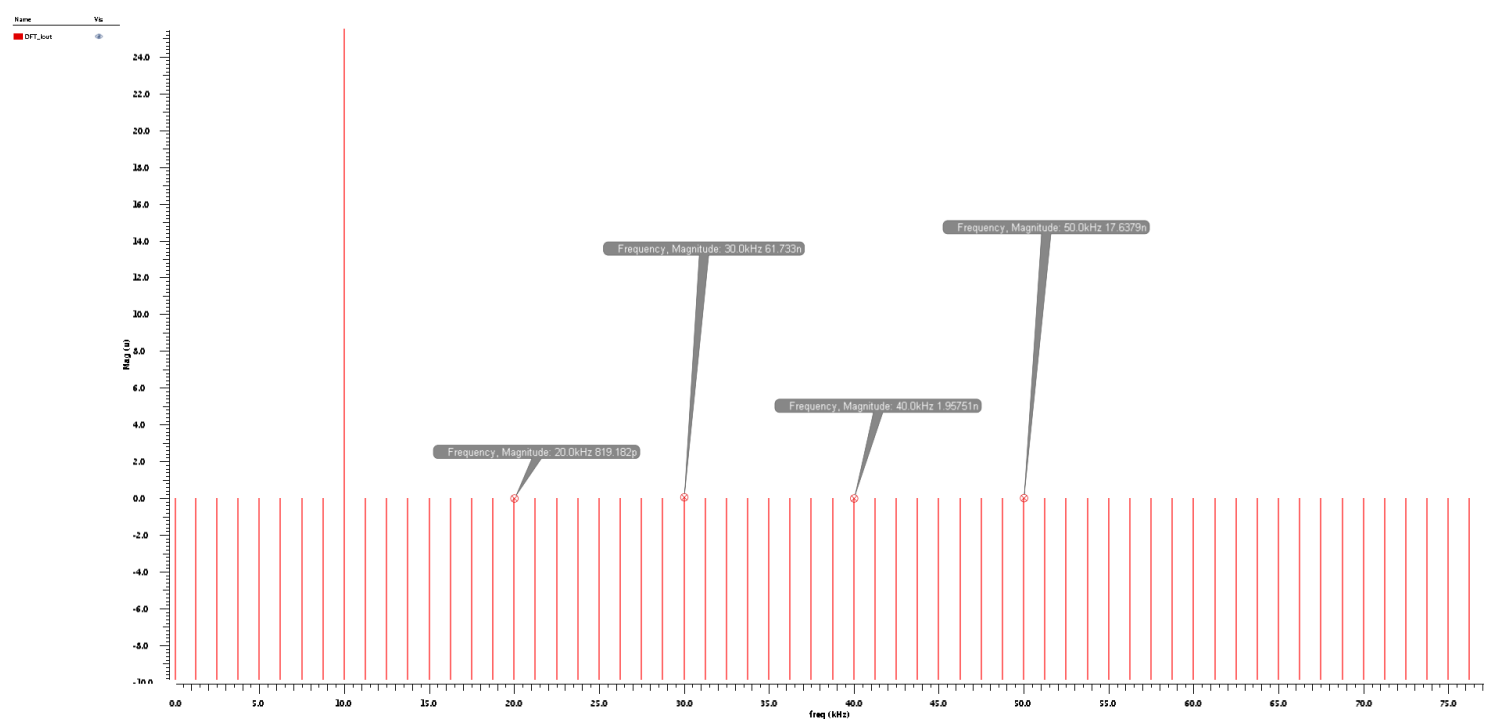

Figure 3.46: The figure displays the current present in the harmonics of the fundamental frequency of the input. Inference can be made that the current in the even harmonics are heavily suppressed compared to the odd harmonics of the fundamental freqency.

of the fundamental frequency are

\begin{tabular}{|c|c|}
\hline Harmonic frequency & Magnitude \\
\hline $20 \mathrm{kHz}$ & $819.1 \mathrm{pA}$ \\
\hline $30 \mathrm{kHz}$ & $61.7 \mathrm{nA}$ \\
\hline $40 \mathrm{kHz}$ & $1.9 \mathrm{nA}$ \\
\hline $50 \mathrm{kHz}$ & $17.6 \mathrm{nA}$ \\
\hline
\end{tabular}

Table 3.2: Output current in the harmonics

Total harmonic distortion (THD) in the case of a current source can be calculated 
by the equation

$$
\text { THD }=\frac{\sqrt{\sum I_{\text {harmonic }}^{2}}}{I_{\text {fundamental }}} \quad x \quad 100
$$

Substituting the currents in the harmonics of the fundamental frequency in the equation 3.40 results in a total harmonic distortion is

$$
T H D=0.06 \%
$$

\section{Current vs. Frequency}

Understandably, the current source is desired to function is the frequency range of interest, which is below $100 \mathrm{kHz}$ for determination of bioimpedance in this case. If the inputs of mirrored modified Howland current source is DC biased at $750 \mathrm{mV}$, for an $\mathrm{AC}$ input of $100 \mathrm{mV}$ the peak output current is observed to be $99 \mu \mathrm{A}$ from simulations. The simulated output current is close to the calculated current magnitude of $100 \mu \mathrm{A}$. The following $\mathrm{AC}$ analysis output, figure 3.47, shows that the current source is functional for all the frequencies below $100 \mathrm{kHz}$. 


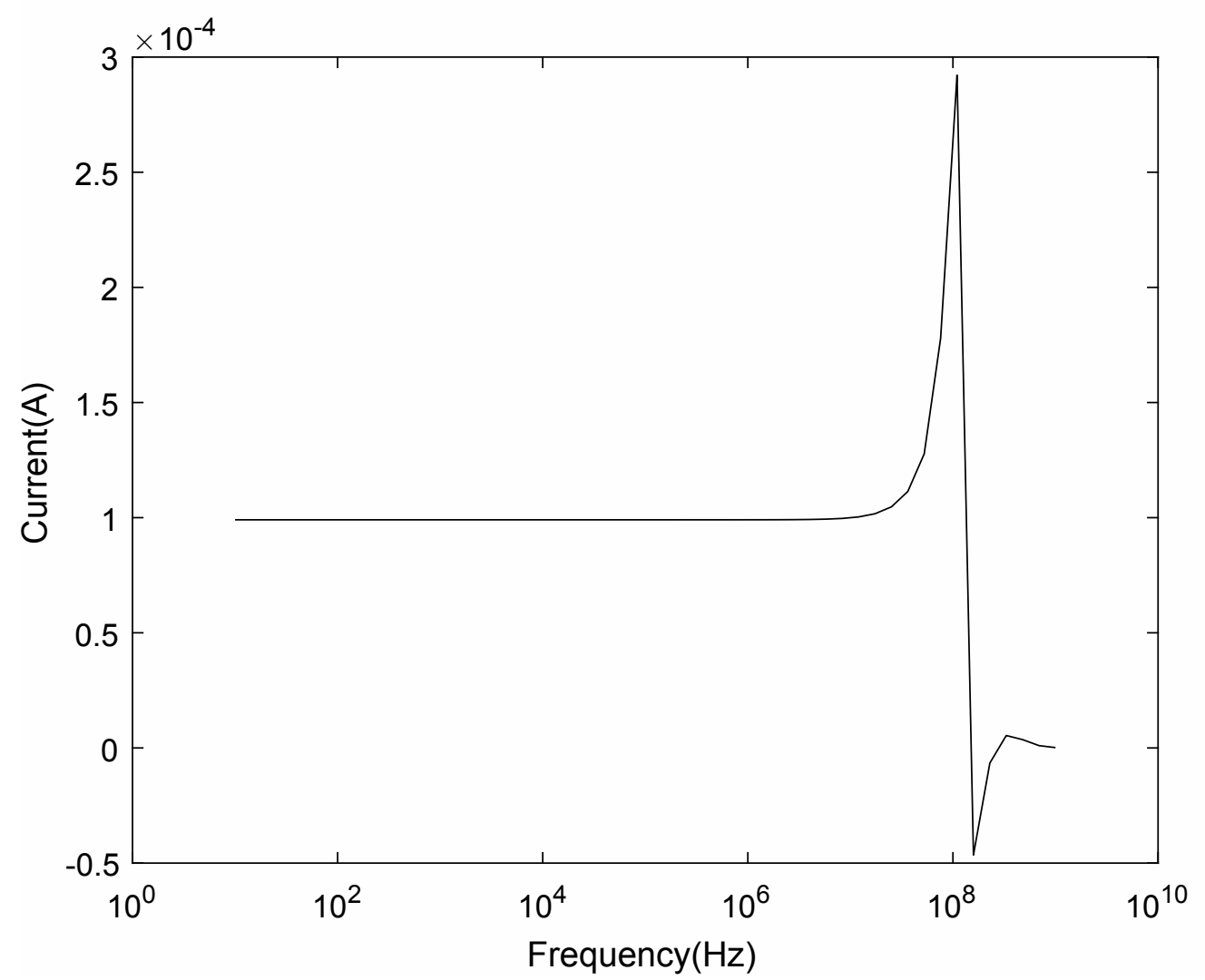

Figure 3.47: The figure illustrates the output current of the mirrored modified Howland current vs. frequency plot obtained through $\mathrm{AC}$ analysis. It is visible clearly that the current source is functional for the frequencies, $1 \mathrm{~Hz}$ to $100 \mathrm{kHz}$, that are of interest to find bioimpedance. The current peaking observed at $109.6 \mathrm{MHz}$ is the current at the natural frequency of oscillation of the current source. 


\section{Power Consumption}

From DC analysis, the static current consumed by the mirrored modified Howland current source is found to be

$$
\text { Current } \text { consumed }=227 \mu \mathrm{A}
$$

Therefore, the power consumed by the instrumentation amplifier is

$$
\text { Power consumption }=1.5 \mathrm{~V} \quad x \quad 227 \mu \mathrm{A}=340.5 \mu \mathrm{W}
$$

\subsection{Instrumentation Amplifier}

An instrumentation amplifier is a precision voltage amplifier that amplify differential voltages and suppress common mode signals. Depending on the topology, an instrumentation offers common mode rejection much higher than operational amplifiers which make them logical choice when operating in noisy environments or very low voltages.

\section{Circuit Description}

The schematic for an instrumentation amplifier is presented in the figure 3.48. It is a 3 op-amp based instrumentation amplifier which operates at gain set by the gain setting resistor, $R_{g}$. 


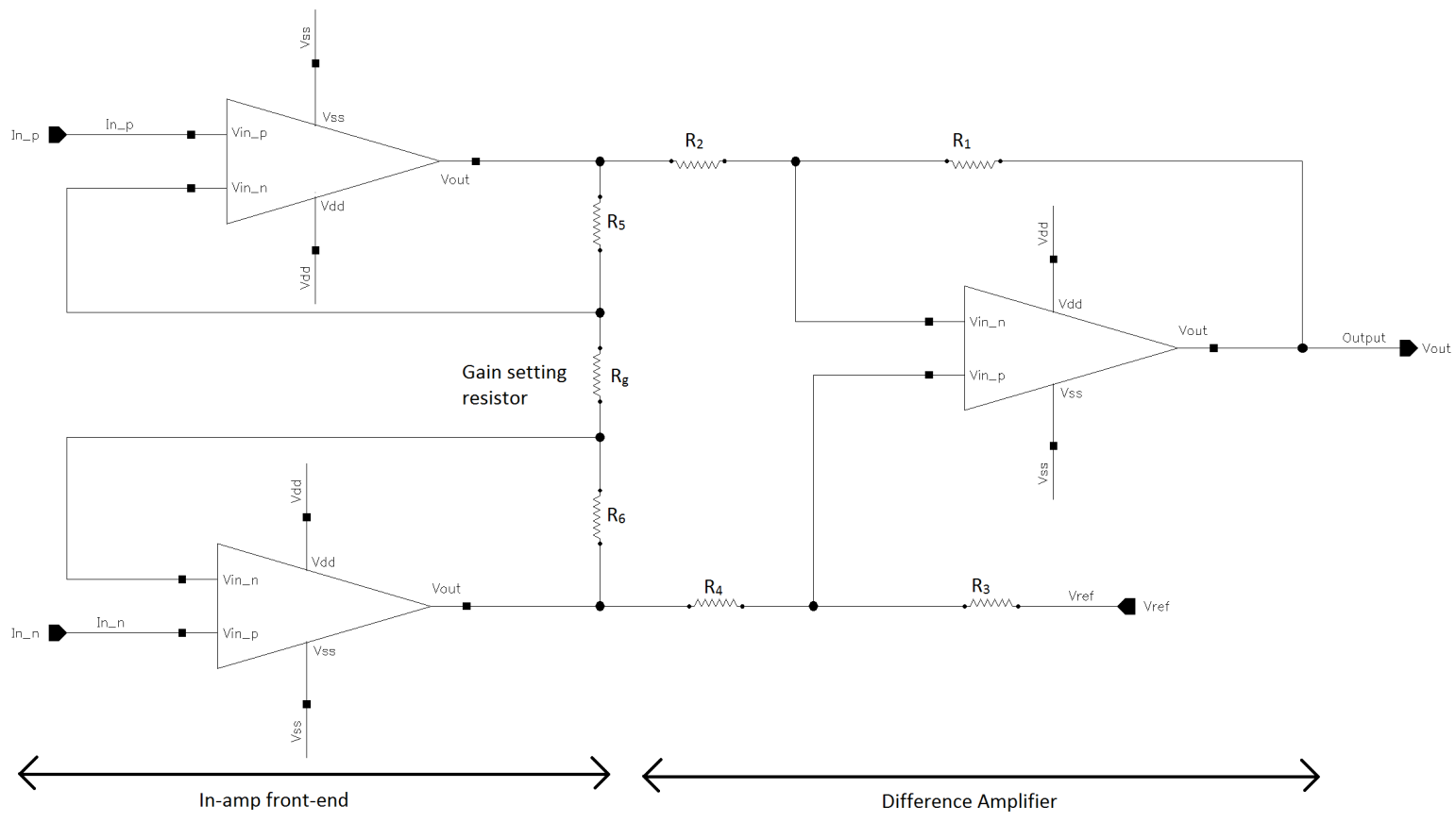

Figure 3.48: The instrumentation amplifier schematic illustrates a 3 op-amp based instrumentation amplifier. In the front-end of the in-amp are two operational amplifier whose gain is set by $R_{5}$ and $R_{g}$. The subsequent stage of the in-amp is a difference amplifier that amplifies the difference between the signals at it's input terminals. 
As seen before, an instrumentation amplifier eliminates any impedance mismatch at the front-end by employing very input impedance operational amplifiers. Electrically speaking, the inputs to the instrumentation amplifier are applied at the non-inverting inputs of the front-end operational amplifiers which are free from connections to any components that pull down it's impedance with respect to ground. The inherent high input impedance of an op-amp thus eliminates any impedance mismatch due to feedback network like in the case of a difference amplifier. Additionally, a MOSFET-input operational amplifier offers a higher input impedance than it's BJT counterpart.

The resistor values are:

\begin{tabular}{|c|c|}
\hline Resistor & Resistance value $(K \Omega)$ \\
\hline$R_{1}$ & 100 \\
\hline$R_{2}$ & 200 \\
\hline$R_{3}$ & 100 \\
\hline$R_{4}$ & 200 \\
\hline$R_{5}$ & 50 \\
\hline$R_{6}$ & 50 \\
\hline$R_{g}$ & Gain dependent \\
\hline
\end{tabular}

Table 3.3: Instrumentation amplifier - Resistor values

\section{Operation}

To the circuit presented in the figure 3.48, when out-phase inputs are applied to the non-inverting input terminal of the front-end operational amplifiers, the negative feedback of the front-end op-amps cause their inverting input terminals 
to follow the input. Thus, the gain setting resistor, $R_{g}$, is applied with out-phase inputs at it's nodes. The difference in potential across $R_{g}$ results in flow of current across itself. This current flows through $R_{5}$ and $R_{6}$ ensuing in a voltage signal at the output node of the op-amps. The establised voltage signals are then fed to the subsequent difference stage as an input that amplifies the difference between the signals and produces an output voltage.

The output voltage of the intrumentation amplifier can be given by the equation 3.41 .

$$
V_{\text {OUT }}=\left(V_{i n+}-V_{i n-}\right)\left(1+\frac{2 R_{5}}{R_{g}}\right)\left(\frac{R_{1}}{R_{2}}\right)
$$

When two out-phase voltage signals of $10 \mathrm{mV}$ amplitude are applied to the instrumentation amplifier at a frequency of $10 \mathrm{kHz}$, the expected output is a voltage signal of amplitude $10 \mathrm{mV}$ if the circuit is operated at unity gain. 


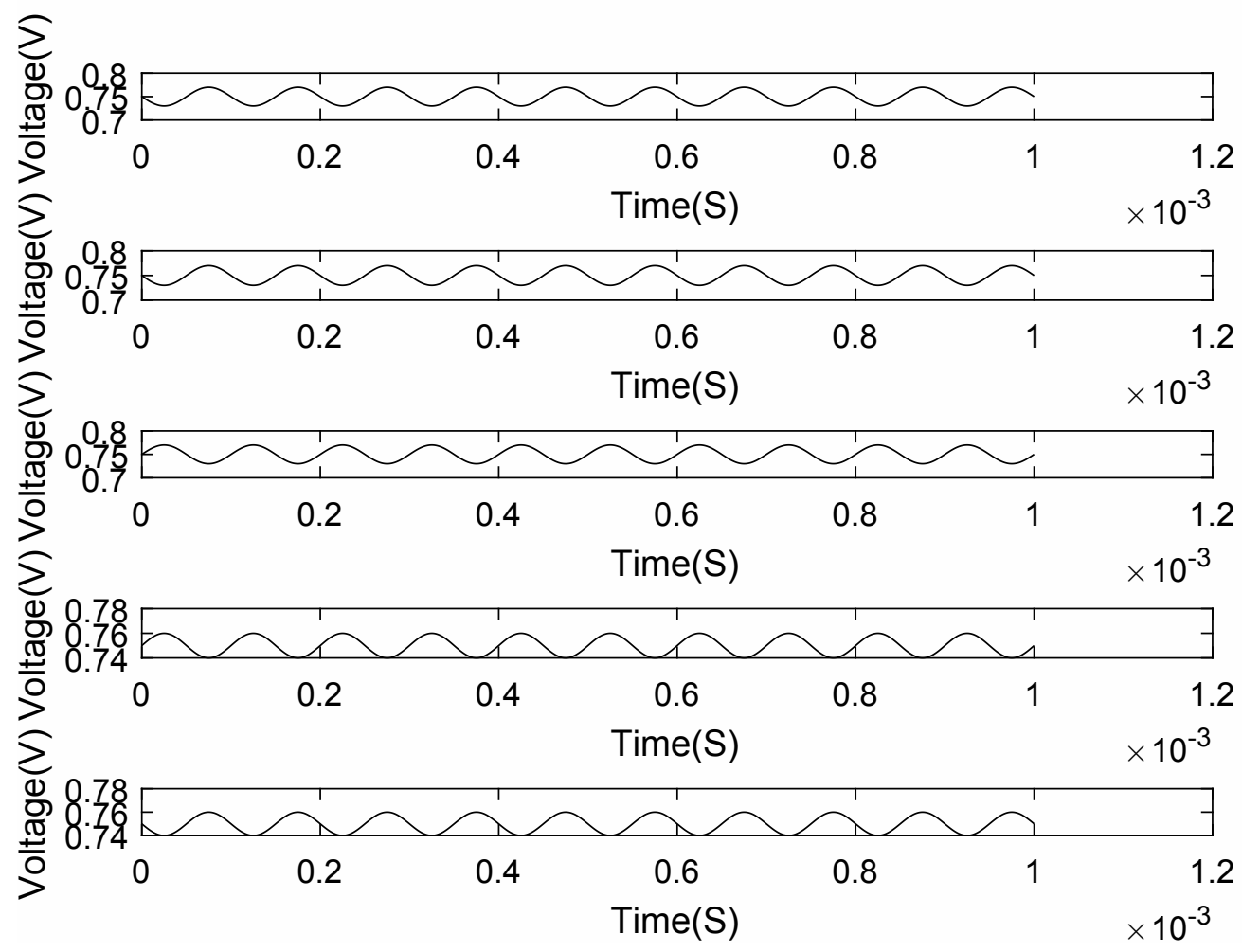

Figure 3.49: From top to bottom: Instrumentation amplifier voltage output signal with an amplitude of $10 \mathrm{mV}$ at a common mode DC of $750 \mathrm{mV}$, Voltage input of amplitude $10 \mathrm{mV}$ at a common mode DC of $750 \mathrm{mV}$ with a frequency $10 \mathrm{kHz}$, Voltage input of amplitude $10 \mathrm{mV}$ out-phase with the other input at a common mode DC of $750 \mathrm{mV}$ with a frequency $10 \mathrm{kHz}$, Intermediate output signal of the amplitude equal to $10 \mathrm{mV}$ at a common mode $\mathrm{DC}$ of $750 \mathrm{mV}$, Intermediate output signal of the amplitude equal to $10 \mathrm{mV}$ at a common mode $\mathrm{DC}$ of $750 \mathrm{mV}$ 
When an input of $10 \mathrm{mV}$ amplitude at a common mode $\mathrm{DC}$ of $750 \mathrm{mV}$ and a frequency of $10 \mathrm{kHz}$ is applied to the instrumentation amplifier an output of $10 \mathrm{mV}$ amplitude is produced as expected. The DC bias of the output signal is set reference voltage which is obtained externally. Therefore, the gain is

$$
G=\frac{10 m V}{10 m V}=1
$$

According to the equation 3.41, an instrumentation amplifier is operated at a unity gain is $R_{g}$ tends to an infinite resistance, in other words open circuit.

The intermediate output signals seen in the figure 3.49 are outputs of the front-end operational amplifiers. These op-amps function as buffers providing the increased input impedance.

\subsubsection{Operating Gain}

Generally, instrumentation amplifiers are used to measure very weak signals, often in noisy environments. The signal thus acquired is amplified by operating the instrumentation amplifier at the gain higher than unity. Unlike operational amplifiers which require two or more resistors to set the gain of the amplifier, the gain of an instrumentation amplifier is set by a single resistor, often referred to as gain setting resistor, indicated by $R_{g}$ in the circuit in the schematic.

Substituting the resistor values in table 3.2, the output voltage in equation 3.41 is simplified to

$$
V_{\text {OUT }}=\left(V_{i n+}-V_{i n-}\right)\left(1+\frac{100 K}{R_{g}}\right)\left(\frac{1}{2}\right)
$$

Simplifying the equation and taking into account the differential input, the gain, 
$\mathrm{G}$, of the instrumentation amplifier can be expressed as

$$
G=1+\frac{10^{5}}{R_{g}}
$$

The relationship between gain setting resistor, $R_{g}$, and the operating gain of the intrumentation amplifier, $\mathrm{G}$, is given by the equation 3.44 .

$$
R_{g}=\frac{10^{5}}{G-1}
$$

$R_{g}$ values for different gains is given by the following table

\begin{tabular}{|c|c|}
\hline Gain & $R_{g}$ \\
\hline 1 & $\infty$ \\
\hline 2 & $100 \mathrm{~K} \Omega$ \\
\hline 5 & $25 \mathrm{~K} \Omega$ \\
\hline 10 & $11.1 \mathrm{~K} \Omega$ \\
\hline 20 & $5.2 \mathrm{~K} \Omega$ \\
\hline 100 & $1.01 \mathrm{~K} \Omega$ \\
\hline 1000 & $100.1 \Omega$ \\
\hline
\end{tabular}

Table 3.4: Instrumentation amplifier - Gain setting resistor values

The figure 3.50 illustrates output and input signals obtained when the instrumentation amplifier is operated at a gain of 2 . When an input of amplitude $10 \mathrm{mV}$ is applied as an input at a common mode DC of $750 \mathrm{mV}$ and a frequency of $10 \mathrm{kHz}$, an output of amplitude $20 \mathrm{mV}$ is observed at the output set at a reference voltage set by an external signal. 


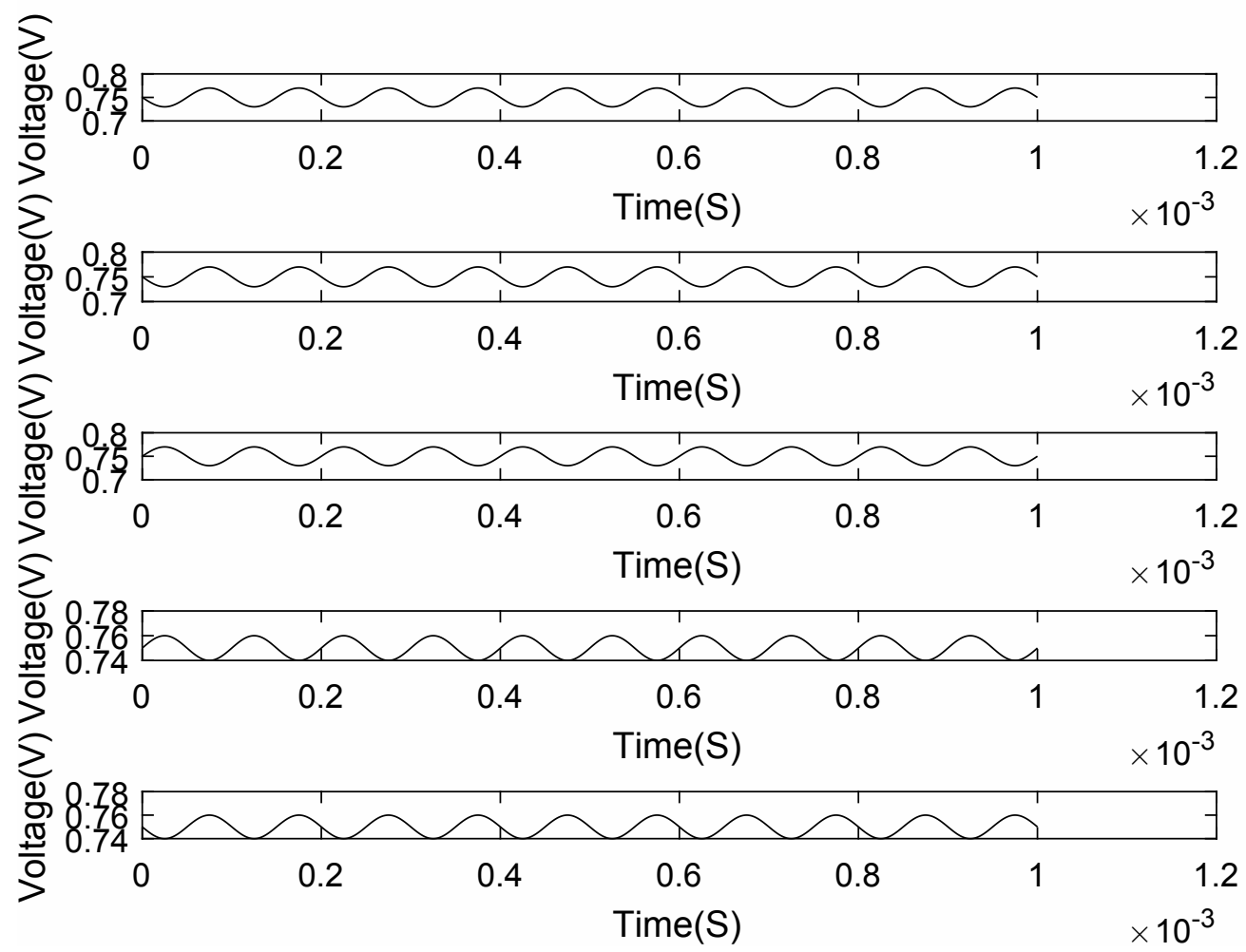

Figure 3.50: From top to bottom: Output voltage signal with amplitude $20 \mathrm{mV}$ and peakto-peak voltage of $40 \mathrm{mV}$, voltage input signal of amplitude $10 \mathrm{mV}$ and peak-to-peak voltage of $20 \mathrm{mV}$, Out-phase voltage input signal of amplitude $10 \mathrm{mV}$ and peak-to-peak voltage of $20 \mathrm{mV}$ 


\subsubsection{Common Mode Rejection Ratio}

Mathematically, common mode rejection ratio (CMRR) is the ratio of differential gain, $A_{D}$, to the common mode gain, $A_{C}$, of the circuit.

$$
C M R R=\frac{A_{D}}{A_{C}}
$$

Common mode signals are suppressed not only by the front-end operational amplifiers by also by the subsequent difference amplifier stage of the instrumentation amplifier. Therefore, an increase in common mode rejection is observed when compared to individual op-amps. This also explains that the increase or decrease of operating gain of the circuit has no effect on the common mode rejection. However, increasing operating gain of the instrumentation amplifier increases CMRR and vice-versa.

For a common mode input of amplitude $10 \mathrm{mV}$, peak-to-peak voltage of $20 \mathrm{mV}$, at $750 \mathrm{mV}$ common mode DC bias and frequency of $10 \mathrm{kHz}$ produces an output signal of peak-to-peak voltage $53.7 \mathrm{nV}$. 

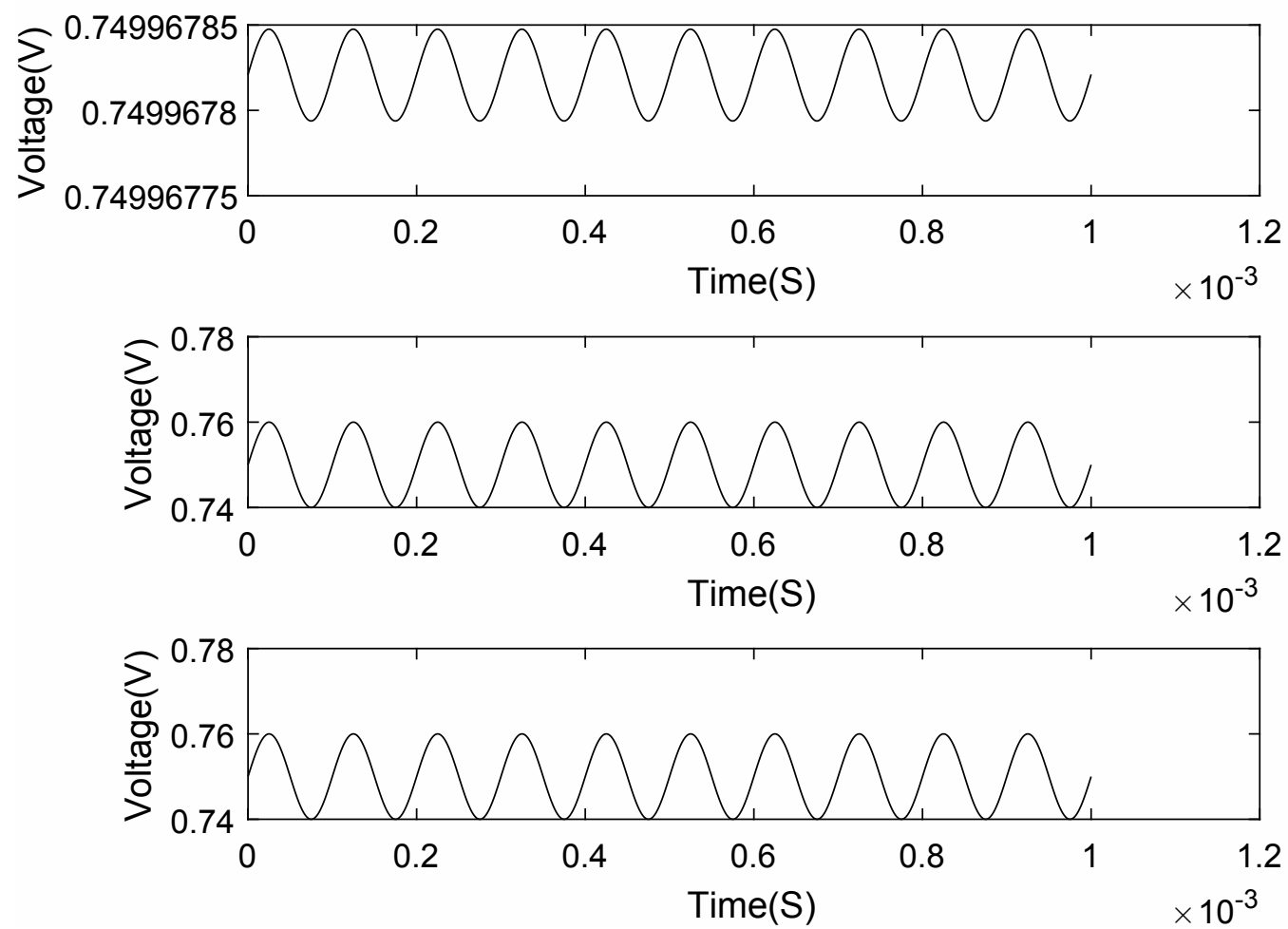

Figure 3.51: From top to bottom: Output signal with peak-to-peak voltage of $53.7 \mathrm{nV}$ at a DC bias of $750 \mathrm{mV}$, common mode input signal with amplitude of $10 \mathrm{mV}$ at a $\mathrm{DC}$ bias of $750 \mathrm{mV}$ and frequency of $10 \mathrm{kHz}$, common mode input signal with amplitude of $10 \mathrm{mV}$ at a DC bias of $750 \mathrm{mV}$ and frequency of $10 \mathrm{kHz}$. 
From the simulations, the common mode rejection of the instrumentation amplifier can be calculated as

$$
A_{C}=20 \log \frac{53.7 n V}{20 m V}=-111.4 d B
$$

Based on the above common mode rejection value and the operating gain of the instrumentation amplifier, the common mode rejection ratio is

\begin{tabular}{|c|c|} 
Gain & CMRR \\
\hline 1 & $111.4 \mathrm{~dB}$ \\
\hline 2 & $117.4 \mathrm{~dB}$ \\
\hline 5 & $125.3 \mathrm{~dB}$ \\
\hline 10 & $131.4 \mathrm{~dB}$ \\
\hline 20 & $137.4 \mathrm{~dB}$ \\
\hline 100 & $151.4 \mathrm{~dB}$ \\
\hline 1000 & $171.4 \mathrm{~dB}$ \\
\hline
\end{tabular}

Table 3.5: Gain and CMRR values of the instrumentation amplifier

Though the table 3.3 shows high CMRR for high operating gains, in reality it is frequency dependent as bandwidth reduces for high gains. 


\subsubsection{Non-linearity}

As explained before, non-linearity is deviation of the output from linear curve with slope 1 . Non-linearity of the designed instrumentation amplifier is an important factor in determining bioimpedance. Non-linearity in the operating voltage range can lead to incorrect voltage measurements reducing the accuracy of the bioimpedance system.

Compared to an op-amp, the non-linearity of an instrumentation amplifier is low and hence is another reason for it's usage in lieu of an op-amp based difference amplifier to measure voltages.

For an instrumentation amplifier, linearly varying the positive input from $0 \mathrm{~V}$ to $1.5 \mathrm{~V}$ while the negative input is at a constant $750 \mathrm{mV}$ DC voltage, produces a linear output within the input range of the circuit. The produced output is shown in figure 3.52 .

The figure 3.52 shows how the output changes with linearly changing input. In the figure, the minimum output voltage is observed to be $\sim 270 \mathrm{mV}$ and the maximum output voltage is seen to be $\sim 1.03 \mathrm{~V}$. It is safe to say that the input and output voltage range of the instrumentation amplifier is $270 \mathrm{mV}$ to $1.03 \mathrm{~V}$. The minimum voltage limitation stems from the $R_{3}-R_{4}$ network and the reference voltage of $750 \mathrm{mV}$, whereas the limited output voltage swing imposes the maximum output voltage limitation of $1.03 \mathrm{~V}$ on the instrumentation amplifier.

The slope plot of the output seen in the figure 3.52 is shown in figure 3.53. In the figure 3.53, the constant output slope is indicative of the fact that the output of the instrumentation amplifier is highly linear but with an offset. Since the instrumentation amplifier is designed to measure difference voltages the offset get's 

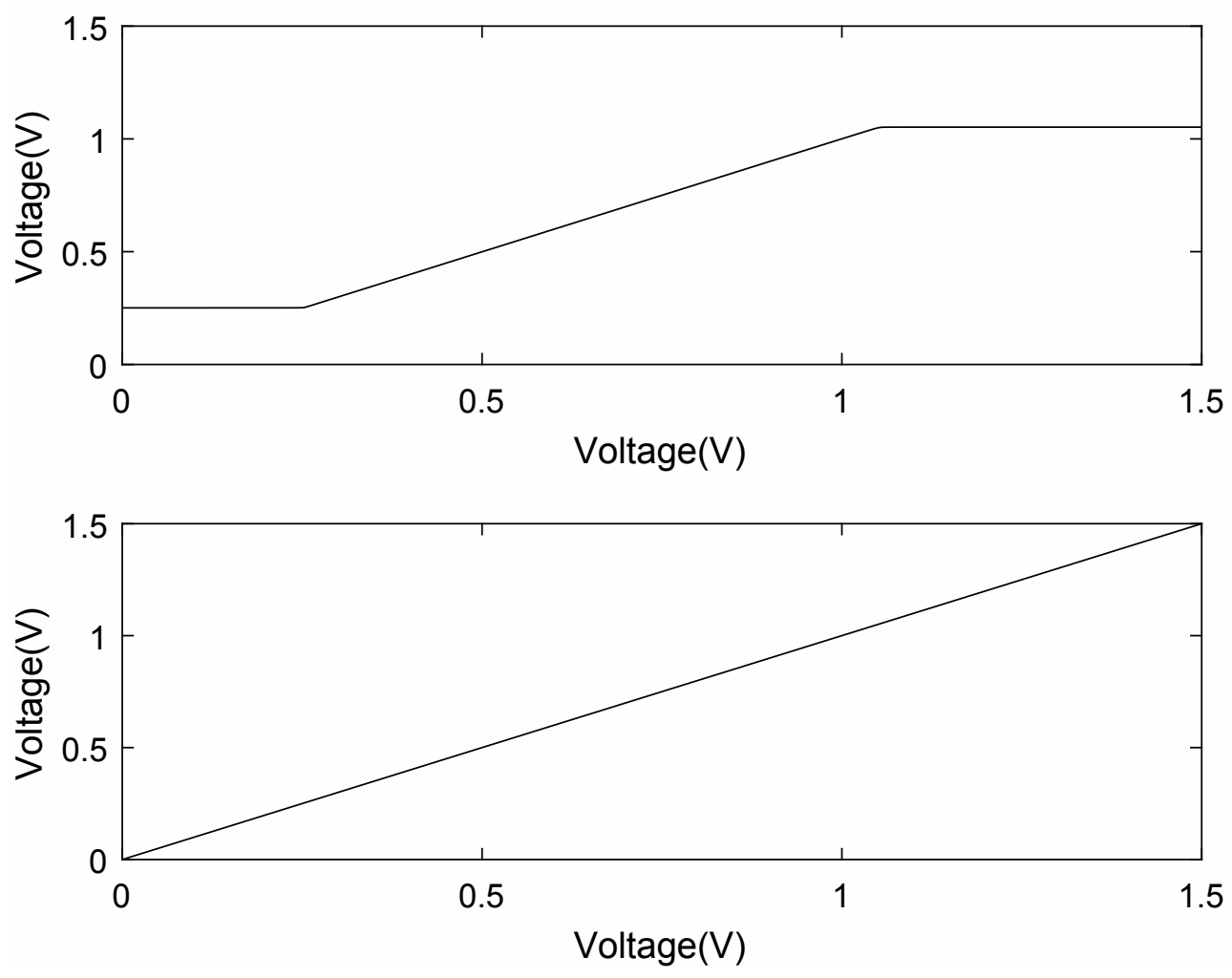

Figure 3.52: Output plot (green) of the instrumentation amplifier with input (red) changing from $0 \mathrm{~V}$ to $1.5 \mathrm{~V}$. The figure also illustrates the output, $749.96 \mathrm{mV}$, observed when both it's inputs are at mid-rail voltage, $750 \mathrm{mV}$.

cancelled and only the difference voltage is seen at the output multiplied by the gain of the circuit. Also, the range of input voltages for which a faithful output is produced is seen to be input voltage range of $270 \mathrm{mV}$ to $1.03 \mathrm{~V}$.

The frequency spectrum of the output for a differential input of $10 \mathrm{mV}$ amplitude at $750 \mathrm{mV}$ DC bias and $10 \mathrm{kHz}$ frequency is seen in the figure 3.54 . The instrumentation amplifier is operated at unity gain with a reference voltage input of $750 \mathrm{mV}$.

From the output spectrum plot, the output voltage at the fundamental and harmonic frequencies is 


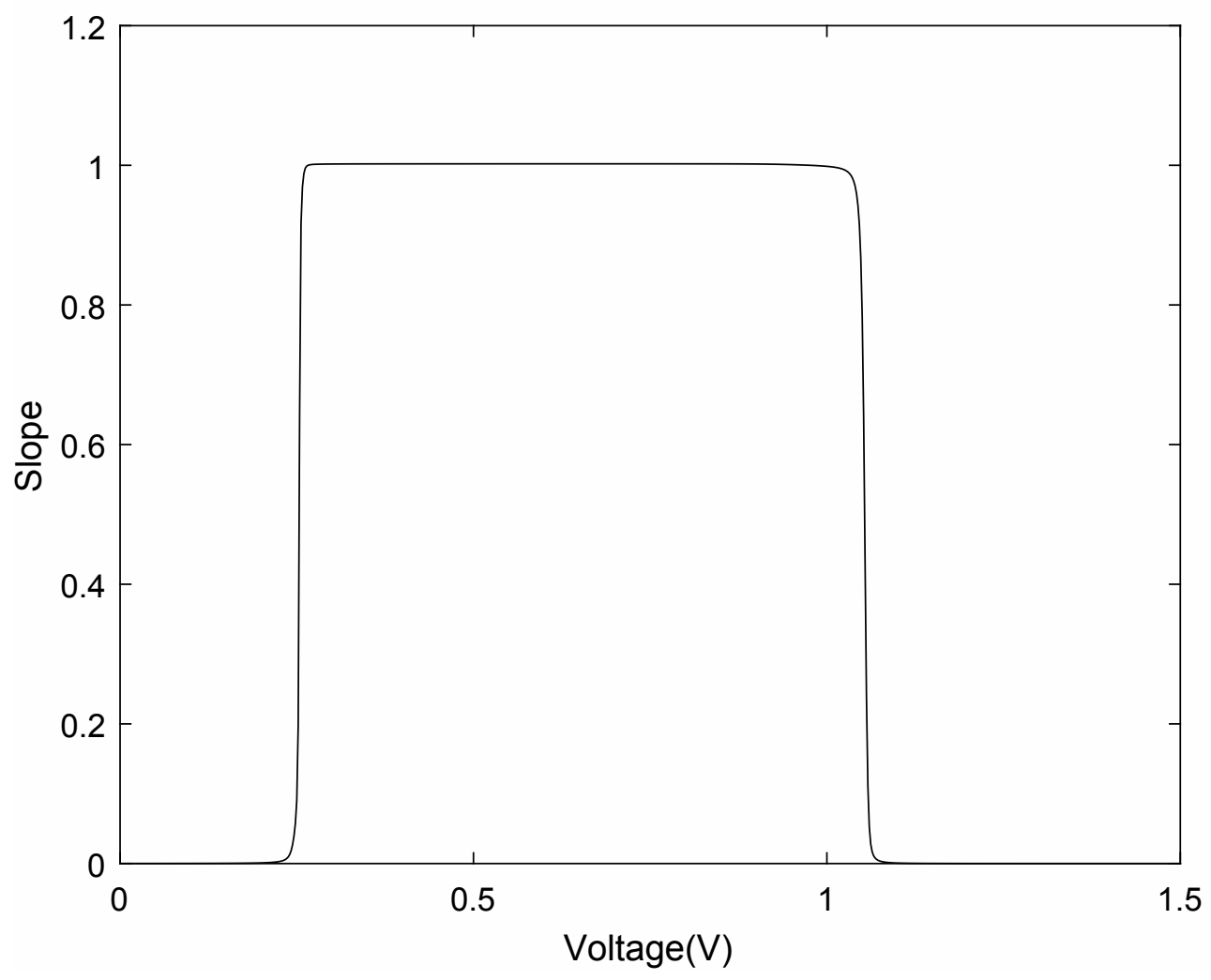

Figure 3.53: The figure illustrates that the slope of the output obtained for an input within the input voltage range of the instrumentation amplifier is only $\sim 1.002$.

The total harmonic distortion (THD) is

$$
\text { THD }=\frac{\sqrt{\sum V_{\text {Harmonics }}}}{V_{\text {Fundamental }}} \times 100 \%=9.5 \times 10^{-5}
$$

The obtained THD is very low compared to the op-amp.

\subsubsection{Offset voltages}

Offset voltage is the additional voltage at the input that causes a $0 \mathrm{~V}$ output. Techniques like chopper stabilization are used in reducing the offset of an instrumenation amplifier which is inherent in the op-amps. This reduced offset voltage is another factor that leads the designer to choose an instrumentation amplifier 


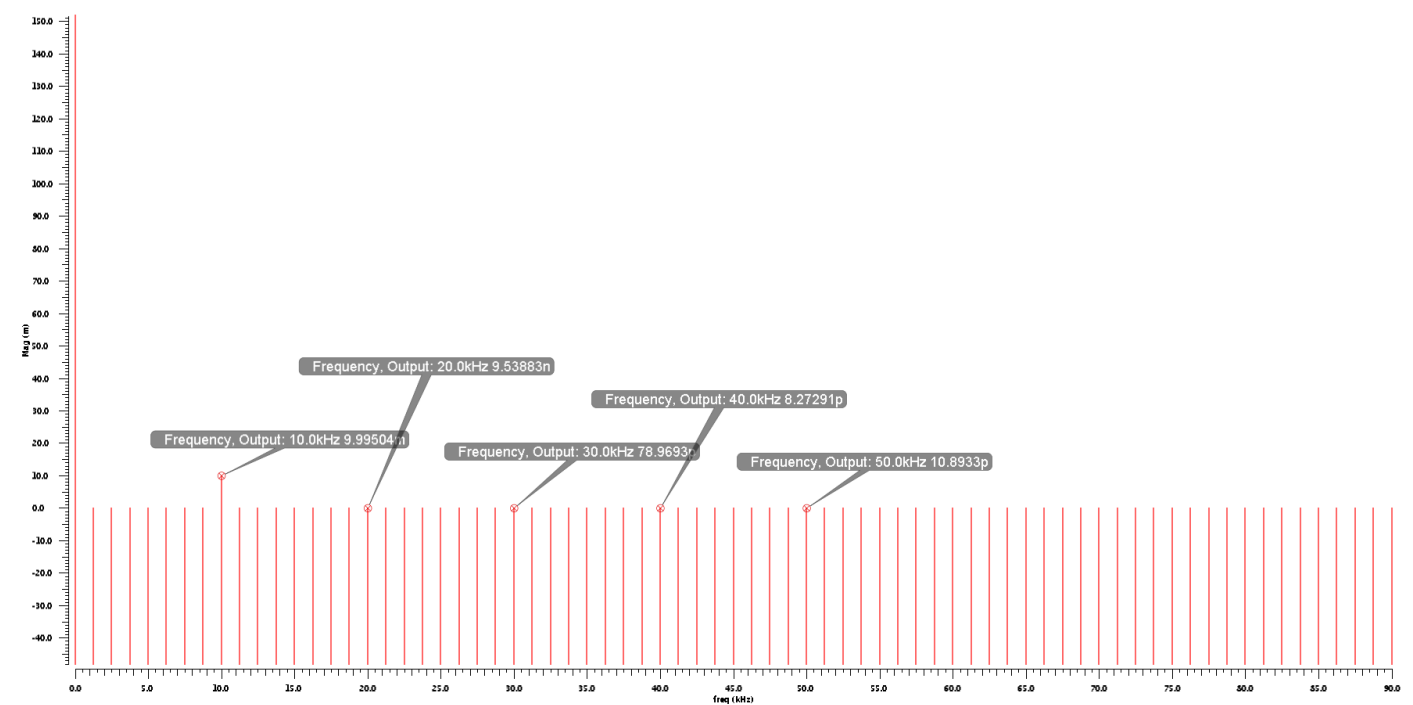

Figure 3.54: The figure clearly illustrates the frequency spectrum for the output of the instrumenation amplifier. The output at $10 \mathrm{kHz}$ fundamental frequency has a magnitude of $9.9 \mathrm{mV}$ indicating unity gain operation of the instrumentation amplifier. The magnitude of output voltage at harmonic frequencies are seen in the figure and are stated in the table ???

over it's op-amp counterpart to measure voltages.

When a constant $750 \mathrm{mV}$ input is applied at both the input terminals of the instrumenation amplifier, the output obtained is $749.96 \mathrm{mV}$. The figure 3.52 illustrates the offset voltage when the instrumenation amplifier is operated at unity gain and the reference voltage is set at $750 \mathrm{mV}$, which is same as the input. The offset voltage therefore is

$$
V_{\text {Offset }}=750 \mathrm{mV}-749.96 \mathrm{mV}=34 \mu \mathrm{V}
$$

The offset voltage measured is solely systematic offset when the circuit instrumentation amplifier is operated at unity gain, and offset due to mismatch is another factor altogether. The offset due to mismatch depends on other factors such as layout, process and temperature variations. While the designer has no control 


\begin{tabular}{|c|c|}
\hline Frequency & Magnitude \\
\hline $10 \mathrm{kHz}$ & $9.9 \mathrm{mV}$ \\
\hline $20 \mathrm{kHz}$ & $9.5 \mathrm{nV}$ \\
\hline $30 \mathrm{kHz}$ & $78.9 \mathrm{pV}$ \\
\hline $40 \mathrm{kHz}$ & $8.2 \mathrm{pV}$ \\
\hline $50 \mathrm{kHz}$ & $10.8 \mathrm{pV}$ \\
\hline
\end{tabular}

Table 3.6: Output voltage in the fundamental and harmonic frequencies

over process and temperature variations, various design and layout techniques can be employed to match devices within the circuit to reduce offsets.

\subsubsection{Power consumption}

From DC analysis, the static current consumed by the instrumentation amplifier is found to be

$$
\text { Current } \text { consumed }=318.5 \mu \mathrm{A}
$$

Therefore, the power consumed by the instrumentation amplifier is

$$
\text { Power } \text { consumption }=1.5 \mathrm{~V} \quad x \quad 318.5 \mu \mathrm{A}=477.7 \mu \mathrm{W}
$$

\subsection{Post Processing Circuit}

When a biological subject is excited by a sinusoidal current, a sinusoidal voltage measured by the instrumentation amplifier. The amplitude of the measured 
voltage depends on the operating gain of the instrumentation amplifier. The sinusoidal signal obtained is rectified and filtered to extract the RMS value of output voltage of the instrumentation amplifier. The RMS voltage thus obtained when divided by the RMS current results in bioimpedance.

$$
Z_{\text {unknown }}=\frac{V_{R M S}}{I_{R M S}}
$$

As discussed before, in case of a diode-based rectifier the threshold voltage of the diode distorts the output signal. For very low threshold voltages and high amplitude input signals, the distortion maybe overlooked sacrificing the accuracy of the output. However, a nominal threshold voltage is a significant barrier for low voltage amplitude signals. To counter the problem with threshold voltage of the diode, a diode-less rectifier is proposed. This rectifier eliminates the possibility of drop in voltage from input to output due to the threshold voltage of a diode.

This section describes the rectifier circuit, transient behavior of the circuit and discussion of the results.

\subsubsection{Circuit Description and Operation}

The circuit designed to rectify an input signal employs transmission gates and operational amplifiers configured as summation circuit and unity gain inverting amplifier.

The figure 3.55 displays the rectifier circuit that is used to rectify output from the instrumentation amplifier.

\section{Half-wave rectifier}

The input to a transmission gate is half-wave rectified when the transmission gate 


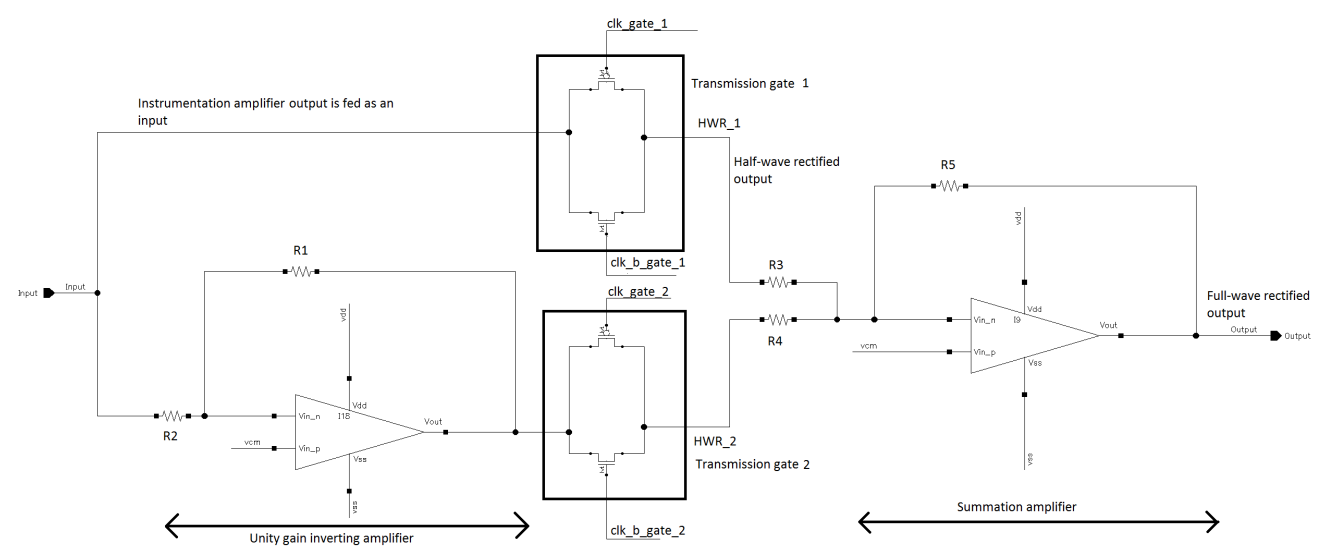

Figure 3.55: In the figure, the 'Input' signal to the transmission gate 1 is the output of the instrumentation amplifier. The output of the transmission gate 1 is half-wave rectified output. The input applied to a unity gain inverting amplifier undergoes an inversion in phase, is halfwave recitfied by the transmission gate 2 and is summed with half-wave rectified output from before. The output that is obtained from the summation amplifier is fully rectified.

is supplied with a precisely timed clock signal.

Transient behavior of a half-wave transmission gate based rectifier, referred to as half-wave gate rectifier, is demonstrated in the figure 3.56. In the gate rectifier schematic, an input signal of $100 \mathrm{mV}$ amplitude at a common mode DC bias of $750 \mathrm{mV}$ and frequency of $10 \mathrm{kHz}$ applied as an input to the transmission gate 1 . Clock and complementary clock signals of frequency equal to the input frequency switches the transmission gate 'on' and 'off'. When the gate is switched 'on' the input signal is passed to the output, and blocked if the gate is switched 'off'.

The half-wave rectified output signal, the input signal along with the clock signals are seen in the figure 3.56. The negative half cycle of the input is passed to the output and the positive half cycle of the input is 'rectified'. The positive half cycle is rectified to facilitate the phase inversion in the final summation stage of the rectifier. By changing the phase of the clock signals one can rectify the negative half 

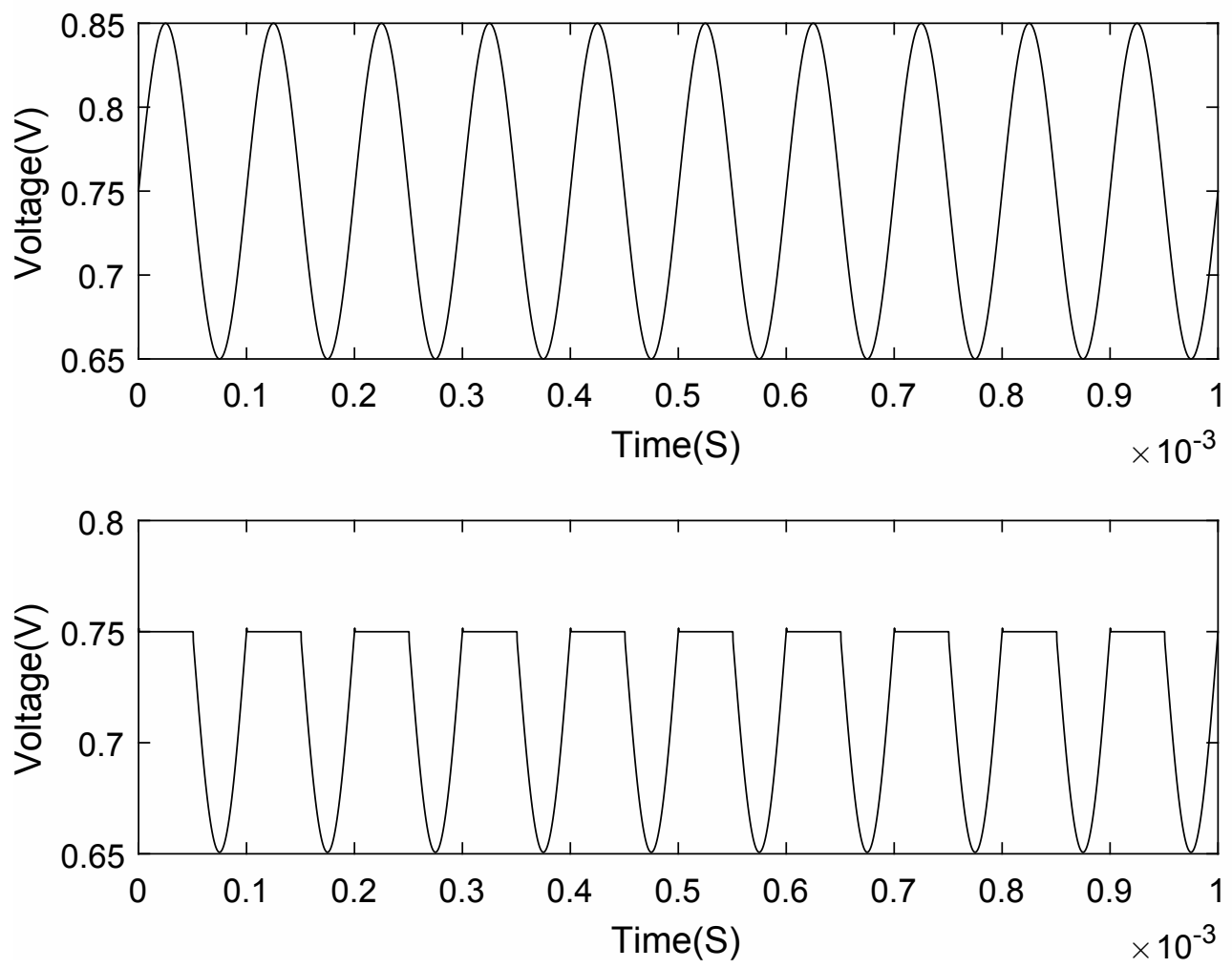

Figure 3.56: From top to bottom: Half-wave rectified output with a maximum voltage of $750 \mathrm{mV}$ and a minimum voltage of $650 \mathrm{mV}$, input signal with a peak-to-peak voltage of $200 \mathrm{mV}$ at a common mode DC bias of $750 \mathrm{mV}$, clock signal applied to the pmos transistor of the transmission gate with $1.5 \mathrm{~V}$ and $0 \mathrm{~V}$ as logic high and low voltages respectively, complementary clock signal applied to the nmos transistor of the transmission gate with $1.5 \mathrm{~V}$ and $0 \mathrm{~V}$ as logic high and low voltages respectively.

cycle and obtain only the positive half cycles of the input signal at the output.

\section{Full-wave rectifier}

Full-wave rectification can be performed by summing two half-wave rectified signals.

Fully rectified output is obtained at the output of an inverting summation amplifier. To account for the inversion in phase during this stage, the half-wave rectified inputs applied to this stage are outputs from transmission gate 1 and transmission gate 2 . The transmission gate 2 rectifies inverted input obtained from unity gain 
inverting amplifier. Thus, the outputs from the transmission gates are half-wave rectified signals with a 90 degree phase shift.

The figure 3.57 illustrates the response of the designed full-wave rectifier circuit to transient input signals.

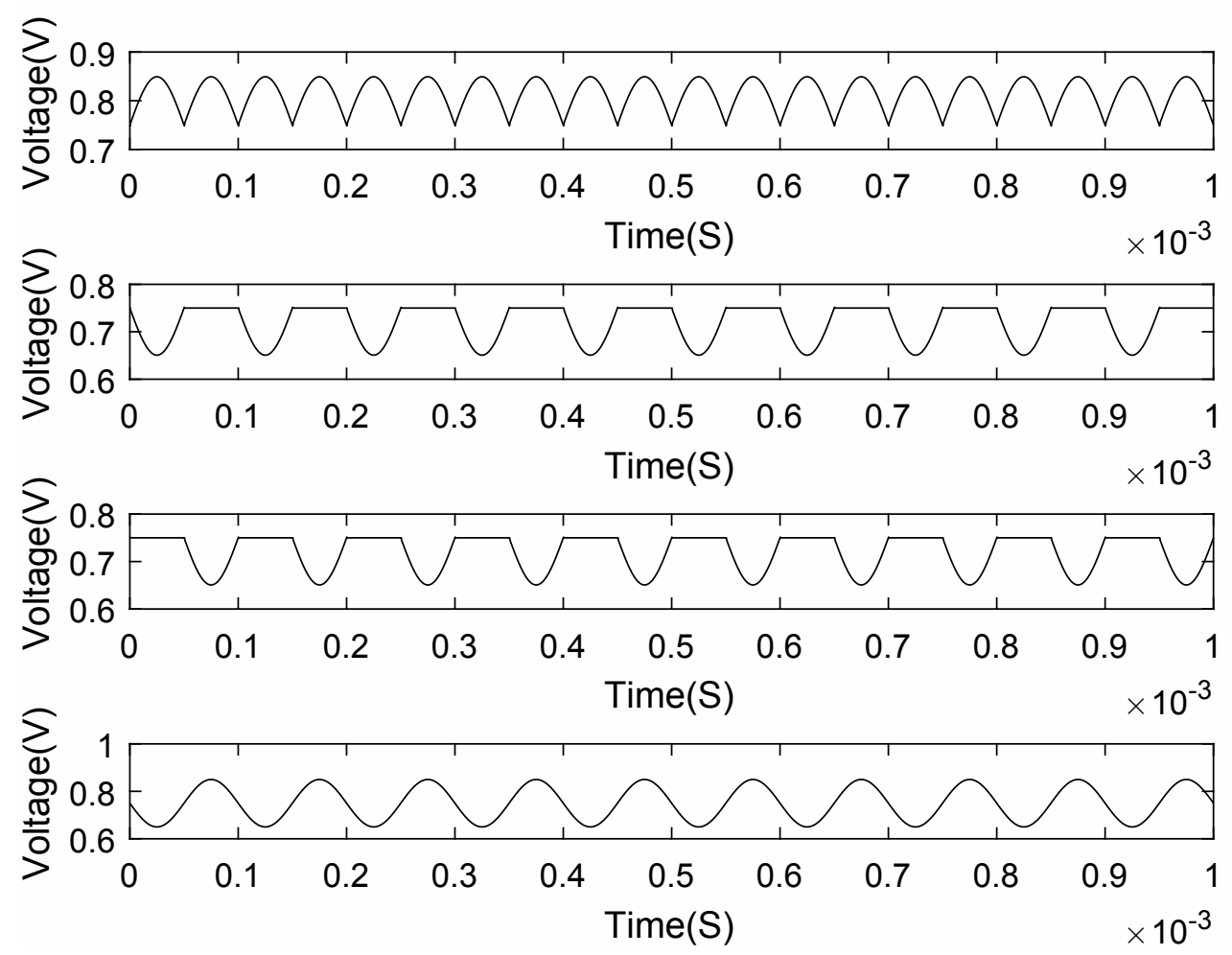

Figure 3.57: From top to bottom: Full-wave rectified output with a maximum voltage of $850 \mathrm{mV}$ and a minimum voltage of $750 \mathrm{mV}$, input signal with a peak-to-peak voltage of $200 \mathrm{mV}$ at a common mode $\mathrm{DC}$ bias of $750 \mathrm{mV}$, half-wave rectified output from the transmission gate 1, half-wave rectified output from the transmission gate 2, output signal obtained from unity gain inverting amplifier. Not shown in the figure are clock signals applied to the pmos and nmos transistors of the transmission gates which have $1.5 \mathrm{~V}$ and $0 \mathrm{~V}$ as logic high and low voltages respectively.

The maximum voltage the fully rectified output seen in the figure is $850 \mathrm{mV}$ and the common mode DC of the output signal is $750 \mathrm{mV}$. The maximum and minimum voltage of the input signal is $850 \mathrm{mV}$ and $650 \mathrm{mV}$ respectively. The common 
mode DC bias of the input signal is $750 \mathrm{mV}$. These values clearly indicate that there is no loss in input voltage during transmission of the input signal to the output.

\subsubsection{Discussion}

In this section, the designed diode-less rectifier is discussed in contrast with the drawbacks of a diode-based rectifier.

The major drawbacks of a diode-based rectifier are

a. Barrier voltage or threshold voltage of a diode

b. Unidirectional nature of a diode

c. High bias currents in a diode.

\section{Barrier Voltage of a diode}

The barrier voltage or threshold voltage of the diode in a diode-based rectifier imposes restrictions on the input signal voltage levels. A diode with a considerable threshold voltage cannot be used for low voltage operation.

Traditionally, the threshold voltage of a silicon diode is $700 \mathrm{mV}$. Germanium diodes and Schottky diodes have a threshold voltage that is approximately equal to $300 \mathrm{mV}$ and $0.2 \mathrm{~V}$. These values prevent them from being used for low voltage operations where the small signals have an amplitude barely equal to $200 \mathrm{~V}$. Even if large currents, say $700 \mu \mathrm{A}$ at $100 \mathrm{kHz}$, were to be injected into a biological subject and the whole-body impedance, assuming the impedance is $500 \Omega$, is measured, a $350 \mathrm{mV}$ amplitude sinusoidal voltage signal is acquired by an instrumentation amplifier. When the output of the instrumentation amplifier is applied to a diode-based rectifier with a diode whose threshold voltage is, say $200 \mathrm{mV}$, a 
distorted output (clipped) signal is generated. Whereas, the output of a diode-less rectifier is undistorted (not clipped). The distorted output of a diode-based rectifier and the undistorted output of a diode-less rectifier are seen in the following figure 3.58 .
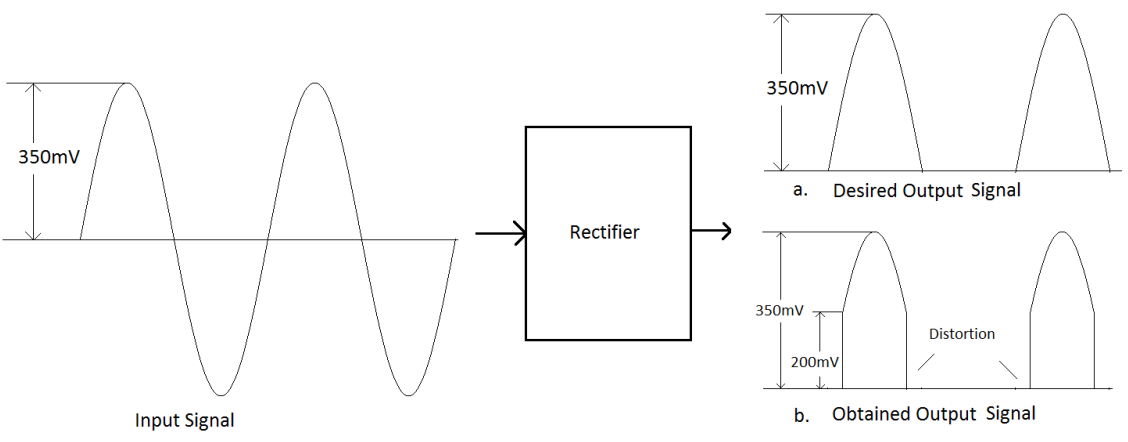

Figure 3.58: For an $\mathrm{AC}$ input of amplitude $350 \mathrm{mV}$, the output of a diode-based rectifier is seen in the figure. The desired output signal (a) is the undistorted output signal obtained using a diode-less rectifier. The distorted output signal (b) is the output signal obtained using a diode-based rectifier.

b. A diode is unidirectional in nature. This implies that when an AC voltage signal is applied a single diode is insufficient to rectify both positive and negative halves of the signal. Often in such cases a network of 4 diodes is employed. This not only increases the layout area of the circuit but also the complexity of the circuit.

A transmission gate is bidirectional and less complicated.

c. The reverse saturation current and forward bias current of a diode are much higher than a transmission gate 'on' current.

In the figure 3.59, the PMOS and NMOS transistors T0 and T1 are switched 'on' by $0 \mathrm{~V}$ and $1.5 \mathrm{~V}$ DC voltages respectively. A very low DC current of magnitude 146.7fA and 661.5fA in the PMOS and NMOS transistors is observed respectively. 


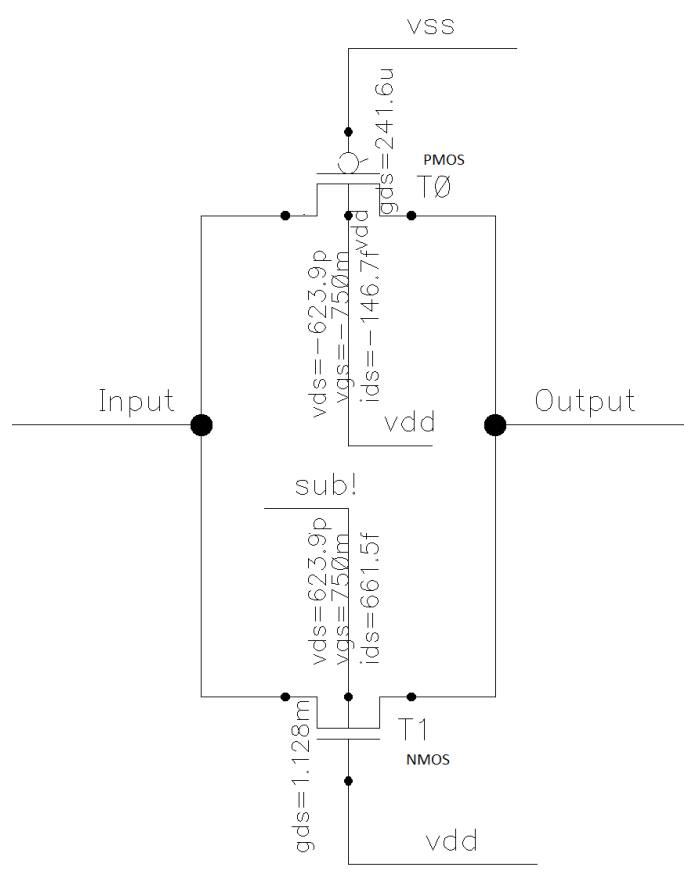

Figure 3.59: The figure displays the result of DC analysis of the transmission gate used in the rectifier. T0 is the PMOS transistor, T1 is the NMOS transistor. The nets 'vdd' and 'vss' switch on NMOS and PMOS respectively and are $1.5 \mathrm{~V}$ and $0 \mathrm{~V}$.

The drain-source voltage observed for both the transistors is a negligible value equal to $623.9 \mathrm{pV}$. Had this voltage been high, the variation in the input and output signal would have be high leading to inaccurate results.

A Very high output conductivity of $241.6 \mu \mathrm{A} / \mathrm{V}$ and $1.1 \mathrm{~mA} / \mathrm{V}$ is registered for PMOS and NMOS transistors respectively. This implies that the 'on' resistance of the transistors is very low, i.e. $4.2 \mathrm{~K} \Omega$ for PMOS transistor and $909 \Omega$ for the NMOS transistor. Since the two transistors are switched 'on' and are operating in triode region, the equivalent 'on' resistance of the transmission gate is equal to the parallel combination of the both resistances. Therefore, equivalent 'on' resistance of the transmission gate is

$$
R_{\text {equivalent }}=909 \Omega \| 4.2 \mathrm{~K} \Omega=747 \Omega
$$


The observed low currents cause very little power consumption and very low drainsource voltage causes negligible error in output.

The 'on' current of a diode is higher than a transmission gate. When 'on' the transistors of the transmission gate operate in triode region and 'on' current is very low because the drain-source voltage is negligible.

The main drawback of a pass transmission gate is limited bandwidth. The figure 3.60 illustrates the $\mathrm{AC}$ response of the circuit for 3 different sets of width and length of tho Nime and Dirne twonniatnme

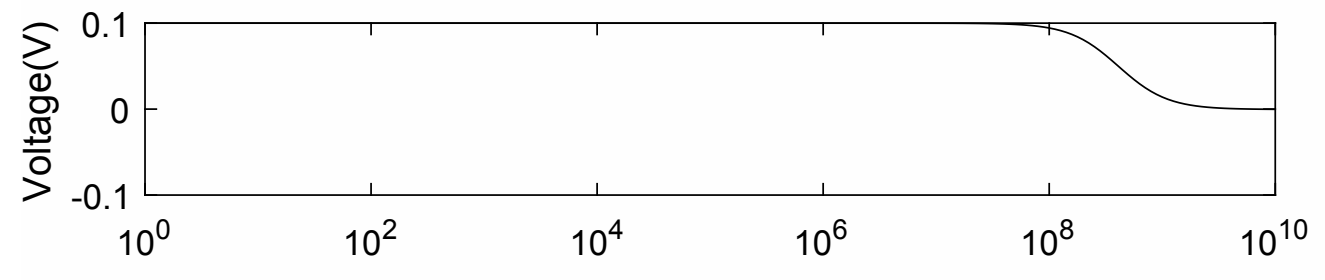

Frequency $(\mathrm{Hz})$
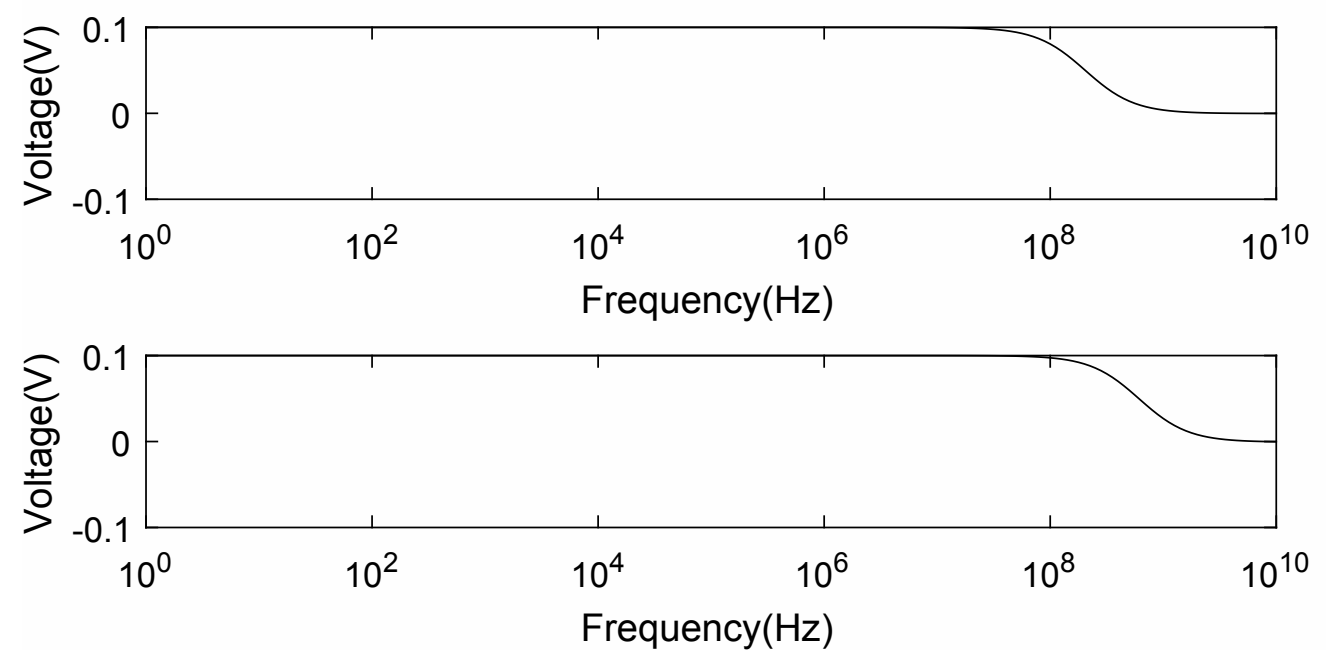

Figure 3.60: Fromn top to bottom: Output vs. frequency plot of the transmission gate whose PMOS and NMOS transistors have $2 \mu \mathrm{m}$ width and $360 \mathrm{~nm}$ length, Output vs. frequency plot of the transmission gate whose PMOS and NMOS transistors have $4 \mu \mathrm{m}$ width and $360 \mathrm{~nm}$ length, Output vs. frequency plot of the transmission gate whose PMOS and NMOS transistors have $6 \mu \mathrm{m}$ width and $360 \mathrm{~nm}$ length 
The following table shows the bandwidth value of the transmission gate for different widths and lengths of it's constituent transistors. The AC plots and the

\begin{tabular}{|c|c|c|}
\hline $\operatorname{PMOS}(\mathrm{m})$ & $\operatorname{NMOS}(\mathrm{m})$ & $\operatorname{Bandwidth}(\mathrm{Hz})$ \\
\hline$W=2 \mu ; L=360 n$ & $W=2 \mu ; L=360 n$ & $9.1 \mathrm{M}$ \\
\hline$W=4 \mu ; L=360 n$ & $W=4 \mu ; L=360 n$ & $18.3 \mathrm{M}$ \\
\hline$W=6 \mu ; L=360 n$ & $W=6 \mu ; L=360 n$ & $27.3 \mathrm{M}$ \\
\hline
\end{tabular}

Table 3.7: Transmission gate bandwidth

table 3.6 reveal that the pass transmission gates suffer from limited bandwidth. Although the bandwidth increases with increase in width of the transistors, at high frequencies larger transistors cause charge injection into the output due to increased parasitic capacitances. Also, at significantly high frequencies the clock applied at the gates of the pass gate transistors might feed through to the output, a phenomenon known as clock feedthrough. 


\subsection{Bioimpedance System}

Bioimpedance system is designed to find bioimpedance by implementing the whole system, i.e., injecting current and sensing the voltage at various different frequencies.

\subsubsection{Bioimpedance System Schematic}

The individual sub-circuits, that are, the designed current source, instrumentation amplifier and the rectifier circuits are integrated to develop the system that can determine bioimpedance.

Clearly, seen in the figure 3.61, the mirrored modified Howland current source, instrumentation amplifier and the gate rectifiers.

For the purpose of simulation, the current source is designed to inject a current of $100 \mu \mathrm{A}$ into an unknown subject. However, since the resistors of the current source are not placed on-chip the magnitude of output current of the mirrored modified Howland current source can be controlled by the user.

The gain of the instrumentation amplifier can 'programmed' by the gain setting resistor, $R_{g}$, but for the purpose of simulation the gain is 1 . The instrumentation amplifier acquires voltage response of the unknown impedance and produces an output depending on the gain it is operating at.

The gate rectifiers acquire inputs from the instrumentation amplifier. The rectifiers produce a fully rectified output by adding two phase shifted half-wave rectified signals. 

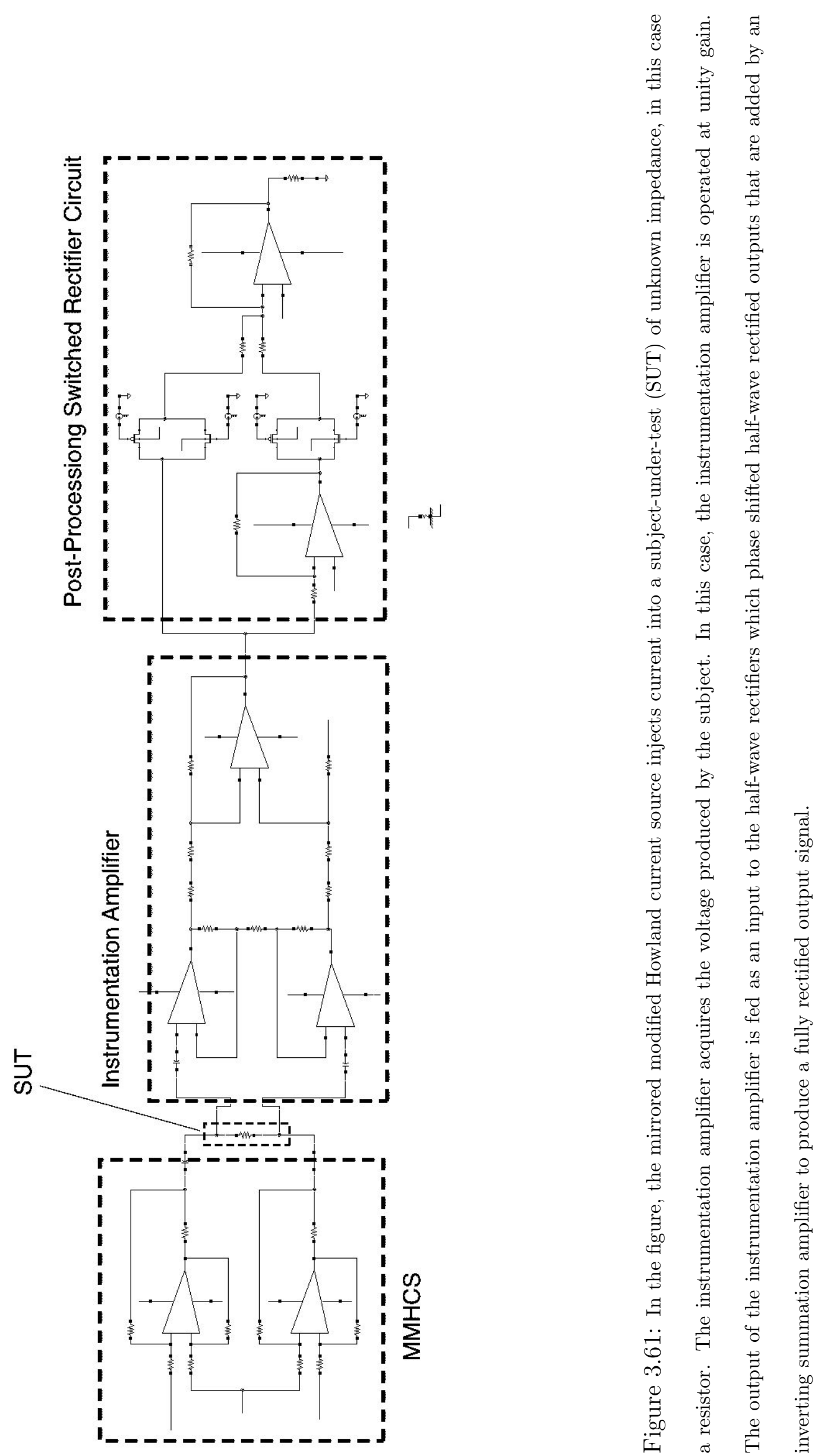


\subsubsection{Simulation and Discussion}

Transient simulation is performed to examine the behavior of the system and verify it's working.

The inputs to the system vis-à-vis current source and other parameters and their values used in performing transient simulation are given in the table below:

\begin{tabular}{|c|c|}
\hline Parameter & Values \\
\hline Power Supply (Vdd) & $1.5 \mathrm{~V}$ \\
\hline Ground & $0 \mathrm{~V}$ \\
\hline Peak AC Input Voltage Signal & $100 \mathrm{mV}$ \\
\hline AC Input Signal Phase & 0 Degrees \\
\hline Input Common Mode Voltage & $750 \mathrm{mV}$ \\
\hline Reference Voltage & $750 \mathrm{mV}$ \\
\hline Frequency & $10 \mathrm{kHz}$ \\
\hline $\mathrm{R}-$ Current Source & $100 \mathrm{~K}-\mathrm{Ohm}$ \\
\hline $\mathrm{r}-$ Current Source & $1 \mathrm{KOhm}$ \\
\hline
\end{tabular}

Table 3.8: Inputs to the BIS system, various parameters and their values used in performing transient analysis. 


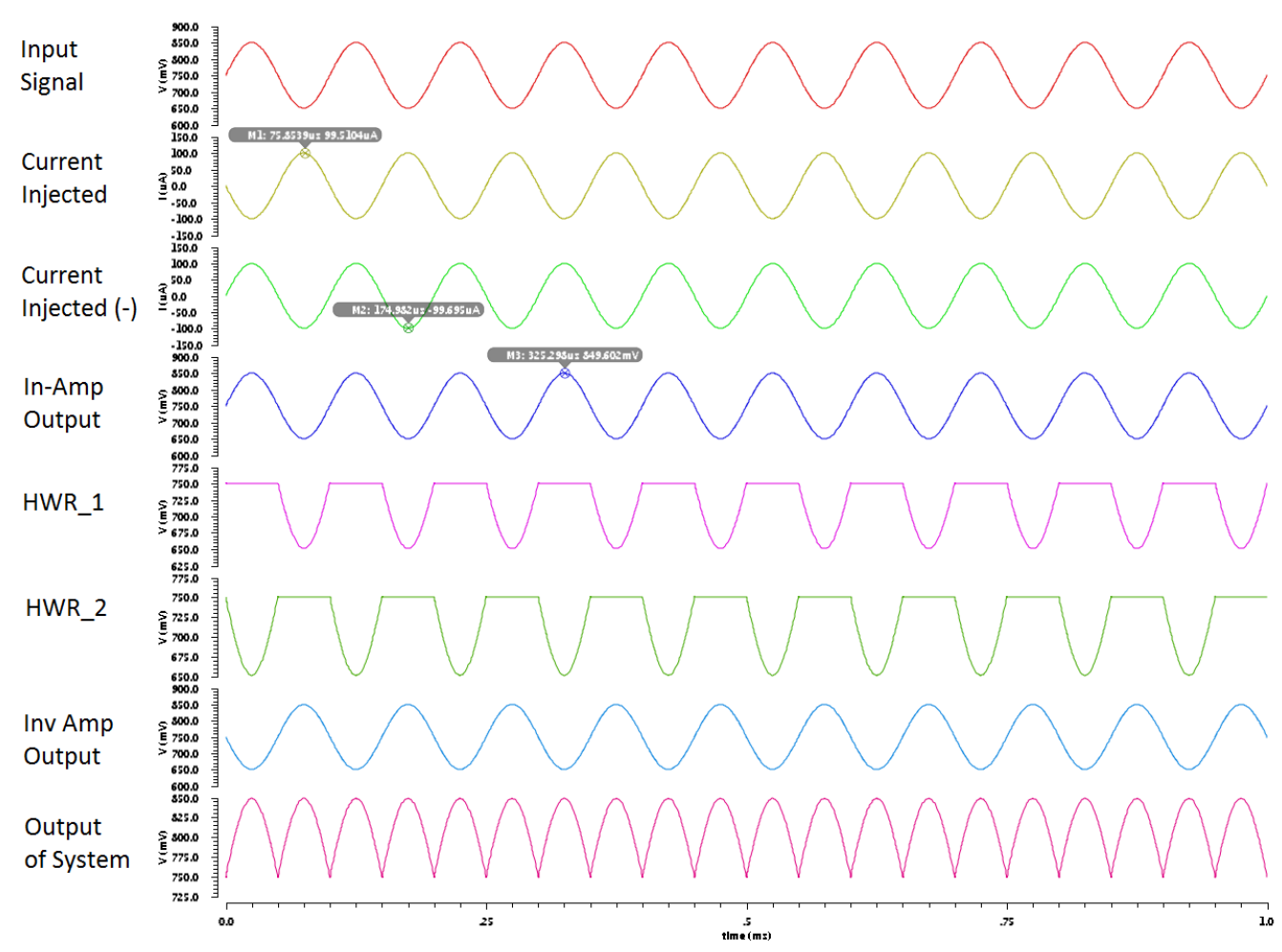

Figure 3.62: From top to bottom: An input signal of $100 \mathrm{mV}$ amplitude at a common mode DC bias of $750 \mathrm{mV}$ and frequency of $10 \mathrm{kHz}$, a $100 \mathrm{muA}$ peak current is injected into the subject(in this case a resistor of unknown impedance), a $100 \mathrm{muA}$ peak current is injected into the resistor(the phase is $180^{\circ}$ with the immediate signal above because in the previous case the current is entering the node and in this case the current is leaving the node), output signal of $100 \mathrm{mV}$ amplitude obtained from the instrumentation amplifier operating at unity gain, half-wave rectified output of the half-wave recitifier, half-wave rectified output of the half-wave recitifier which is $90^{\circ}$ in phase with the other half-wave rectified output, output of the unity gain inverting amplifier, a fully-rectified output signal with a peak amplitude of $100 \mathrm{mV}$ obtained at the output of the full-wave rectifier circuit 
In the figure 3.62 , for an $\mathrm{AC}$ input signal of $100 \mathrm{mV}$ peak voltage and due to the $1 \mathrm{KOhm}$ gain setting resistor of the modified Howland current source, the output current of the current source is approximately $100 \mu \mathrm{A}$. While one modified Howland current source is sourcing the current into the SUT the other is sinking it from the SUT, where the SUT in this case is a $1 \mathrm{KOhm}$ resistor.

The voltage reponse of the SUT is sensed and acquired by the instrumentation amplifier. The instrumentation amplifier is configured for unity gain. Therefore the output voltage signal from intrumentation amplifier is observed to swing from $850 \mathrm{mV}$ to $650 \mathrm{mV}$ at a common model of $750 \mathrm{mV}$, which is set by the input reference voltage.

At this point, since the current injected by the current sources into the SUT is $99.69 \mu \mathrm{A}$ and the voltage output of the instrumentation amplifier is $99.602 \mathrm{mV}$, the unknown impedance is the ratio of RMS voltage acquired and injected RMS current. This results in

$$
Z_{\text {unknown }}=\frac{V_{R M S}}{I_{R M S}}=\frac{70.7 m V}{70.7 \mu}=1 K \Omega
$$

The output from instrumentation amplifier is applied as an input to the rectifier and a fully-rectified output is seen at the output as seen in the figure 3.62 .

\subsection{Layouts}

This section displays the designed layouts and employed layout techniques that aid in the performance of the circuit for the operational amplifier, instrumentation amplifier and the IC. 
The schematic view of an integrated circuit describes the circuit with standard symbols of electrical components. Whereas, the layout of an integrated circuit is a description of the designed integrated circuit with shapes of semiconductor, metals and oxide mainly.

Based on manufacturers, process node and various other factors, most semiconductor technologies comprise of a set of layers. Most used layers are metals, silicon, polysilicon and oxide. An electrical component is fabricated using the layers that are available for the technology. For example, fabricating a transistor involves polysilicon for gate, doped semiconductor substrate for active regions, metals for contacts. Generally, metals are used for wire connections.

Once the design of the layout for a circuit is completed, the layout is subjected to check for rules, layout versus schematic connections, feasibility of manufacturability, etc. and other processes depending on the requirements.

If the layout passes all the checks, the layout data is used to generate a GDSII file, a file format for which contains information information about IC layout artwork and create photomasks, and shipped to the manufacturer.

The designed chip is named ICGCUAUM and sent to CMC for a tapeout. The performance of a fabricated IC with subcircuits instrumentation amplifier and the whole chip layout is not always guaranteed because of process, voltage and temperature variations. However, it is possible to employ special layout techniques to ensure the proper working of the electrical components in the circuit.

The figure 3.63 illustrates the layout of the operational amplifier shown in the schematic 3.1.

The figure 3.64 illustrates the layout of the instrumentation amplifier. 
The figure 3.65 shows the layout of the designed IC, ICGCUAUM.

The figure 3.63 illustrates the layout techniques used to improve the performance of the layout. The differential pair input transistors of both are laid out using common centroid layout technique. The common centroid layout improves matching of transistor by causing symmetrical variations in the transistors due to process variations. However, the common centroid pattern can lead increased parasitics and cause mismatch if the symmetry in routing is not maintained.

ABBA

BAAB.

The differential input routing connects are close to each other to make sure that the effect of noise is similar of the routing metals and is rejected by the operational amplifier.

Also, special care has been taken in laying the transistors to make sure that the orientation of current in the components that need matching is preserved. 


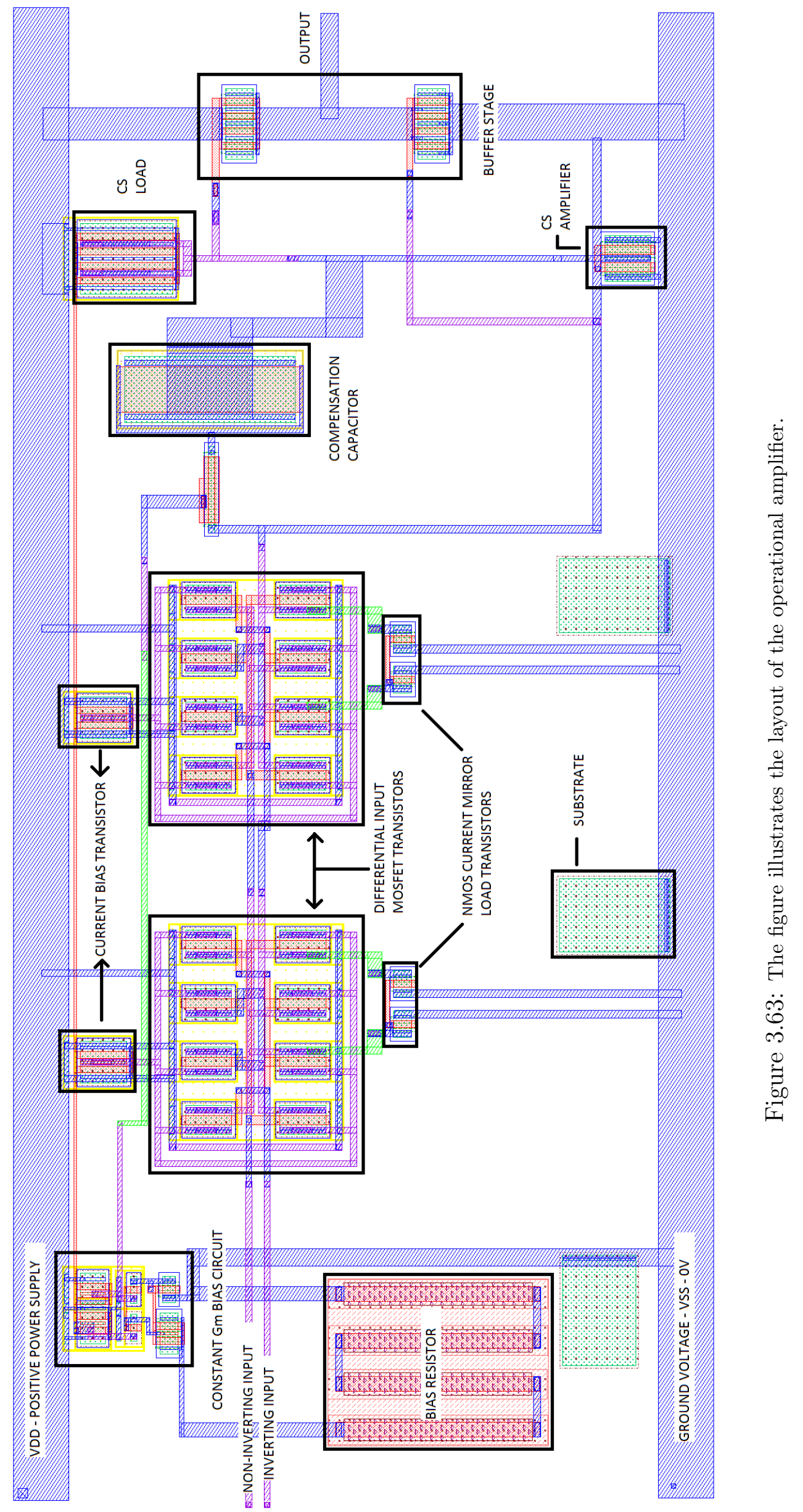




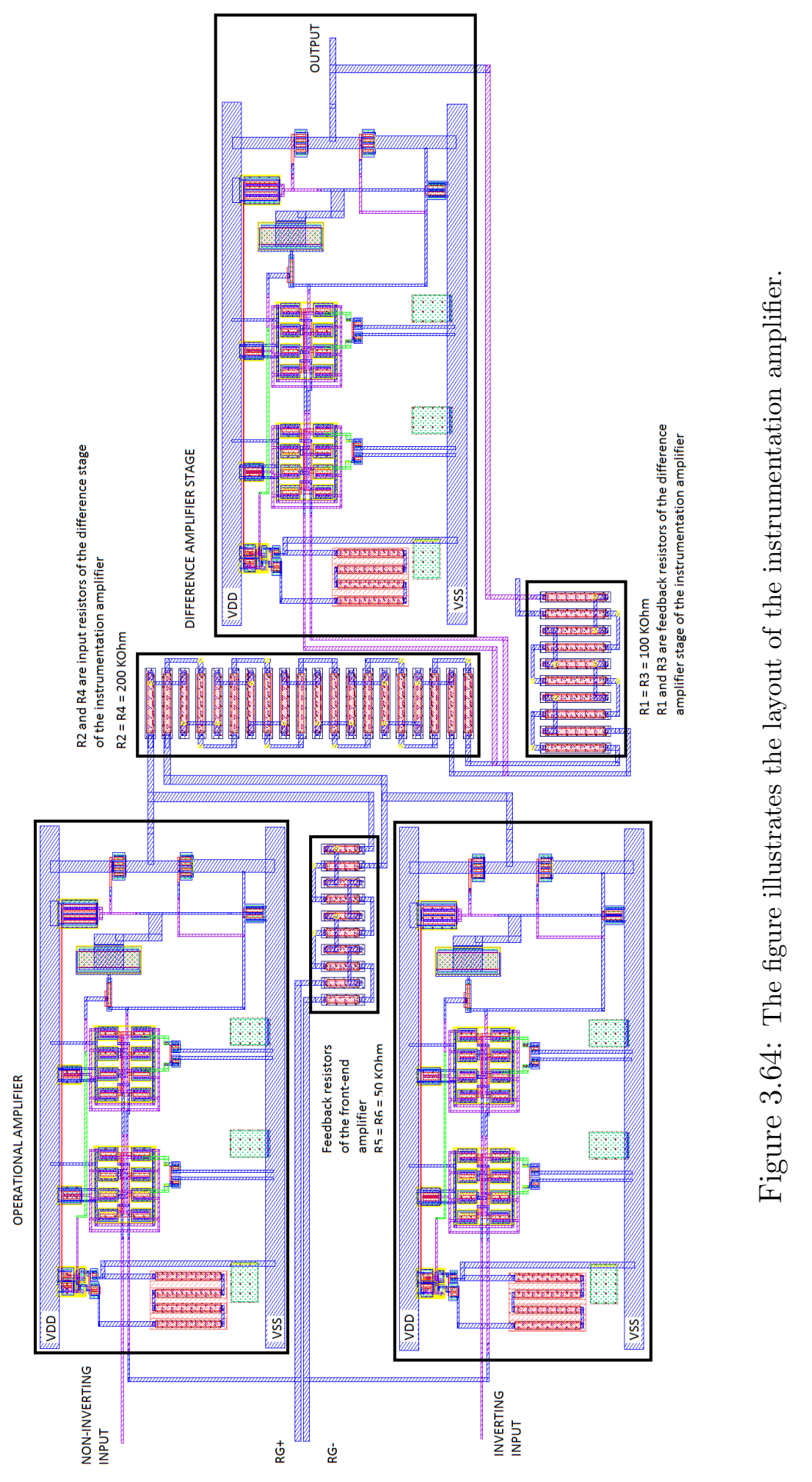




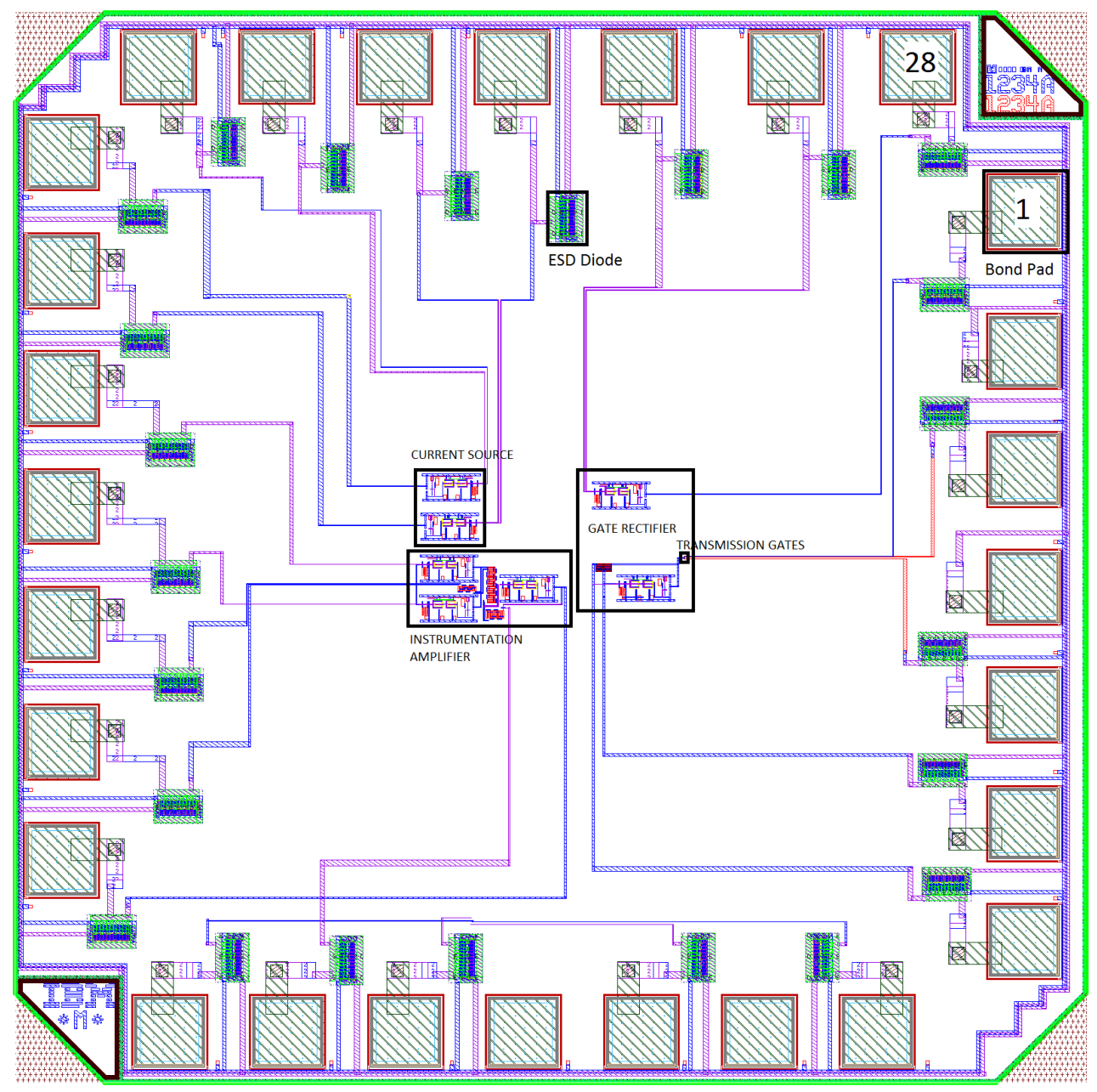

Figure 3.65: The figure illustrates the IC layout of the ICGCUAUM. 


\subsection{Chapter Summary}

This Chapter details information pertaining to the design and implementation of the bioimpedance system and its sub-circuits.

The Chapter begins with the description of the operational amplifier circuit and presents an overview of its working. Then examines the difference in the topology of a differential gain stage with active and current mirror loads, illustrates working of the circuit and explains the source of increased gain of the operational amplifier. The presented transient simulation results testify the theory and understanding of the circuits. This is followed by explaining the operation of the cascaded differential gain stages along with transient simulations.

The designed operational amplifier is characterized by determining through simulations the differential gain, common mode rejection, offset voltages and various other parameters. Critical characteristics of the operational amplifier that directly affect the performance of the other sub-circuits and the system have been discussed at length before going to the other blocks of the system. A good deal of effort has gone into designing and characterizing the operational amplifier because of its direct effect on the performance of the other sub-circuits.

The mirrored modified Howland current source is an op-amp based voltage controlled current source whose performance depends on the performance of the designed op-amp. Before going into mirrored modified Howland current source, the basic Howland current source and the modified Howland current source circuit have been detailed. This is because, while the fundamental understanding of the current source circuit is derived from the basic Howland current source, the mirrored modified Howland current source comprises two modified Howland current 
sources. The theory of operation of the circuits has been stated with necessary mathematical analysis wherever necessary. Simulations attest the theory and validate the mathematical relationships between the input and the output.

The instrumentation amplifier is a closed-loop voltage feedback circuit that can acquire and measure very small voltage signals in noisy environments. The theory of operation of the circuit has been laid out with necessary mathematical relationships that go into designing it. Transient and DC simulations substantiate the concept and characterise the circuit.

A diode-less transmission gate based rectifier, gate rectifier, is used to rectify the signal acquired from the instrumentation amplifier and find out the RMS value of the voltage response of a biological subject. The ratio of RMS voltage of the unknown impedance and the RMS value of the current injected is the value of unknown impedance, in this case, bioimpedance. The concept of diode-less transmission gate based rectification is justified with the help of transient simulations and then the pros and cons of the circuit are discussed in contrast with a diodebased rectifier.

Once the individual pieces of the system are explored, the system built by integrating the sub-circuits and bioimpedance of an unknown impedance is determined through transient simulations. Layouts of the sub-circuits and the chip layout have been illustrated and discussed. Principles and techniques employed in designing layouts have been discussed at the end of the Chapter.

The following table 3.9 outlines the specifications of the designed operational amplifier that are determined through simulations. 


\begin{tabular}{|c|c|}
\hline Specification & Value \\
\hline Supply voltage & $1.5 \mathrm{~V}$ \\
\hline Differential gain @ 10kHz & $85.7 \mathrm{~dB}$ \\
\hline Common mode rejection @ $10 \mathrm{kHz}$ & $-48.4 \mathrm{~dB}$ \\
\hline Common mode rejection ratio & $134.1 \mathrm{~dB}$ \\
\hline UGBW & $677.1 \mathrm{MHz}$ \\
\hline Input common mode range & $\sim(2 \mathrm{mV}, 1 \mathrm{~V})$ \\
\hline Output voltage swing & $\sim(75 \mathrm{mV}, 1 \mathrm{~V})$ \\
\hline Slew rate & $49 \mathrm{MV} / \mathrm{s}$ \\
\hline Non-linearity - Best straight line method & $\begin{array}{l}\text { Max. deviation }=1.5 \mathrm{mV} \\
\text { Min. deviation }=7.5 \mu \mathrm{V}\end{array}$ \\
\hline Offset voltage & $85 \mu \mathrm{V}$ \\
\hline Output Impedace & $33.3 \mathrm{~K} \Omega$ \\
\hline Power consumption & $169.6 \mu \mathrm{W}$ \\
\hline
\end{tabular}

Table 3.9: Operational amplifier simulated specifications 


\section{Chapter 4}

\section{Testing and Debugging}

The Chapter deals with testing of the chip, ICGCUAUM, and its failure analysis. Regarding the results of the testing, the chip failed to produce desired results due to instability in the op-amp. Though the op-amp worked partially with several work-arounds it failed to perform bio-electrical impedance analysis, and hence failed to find bioimpedance. Also, due to internal connections and inaccessibility of intermediate nodes, the instrumentation amplifier and the rectifier could not be implemented.

This Chapter is divided into two sections. The first section consists of information about testing equipment, setup and test sequence. The second section discusses the issues that lead to the failure of the chip. 


\subsection{Testing}

\subsubsection{Chip Setup}

The Design Under Test (DUT) is the chip ICGCUAUM. The chip is glued to a generic Printed Circuit Board (PCB), wire-bonded and tested using appropriate equipment available at Carleton University.

A photograph of the chip bonded to the PCB is shown in figure 4.1.

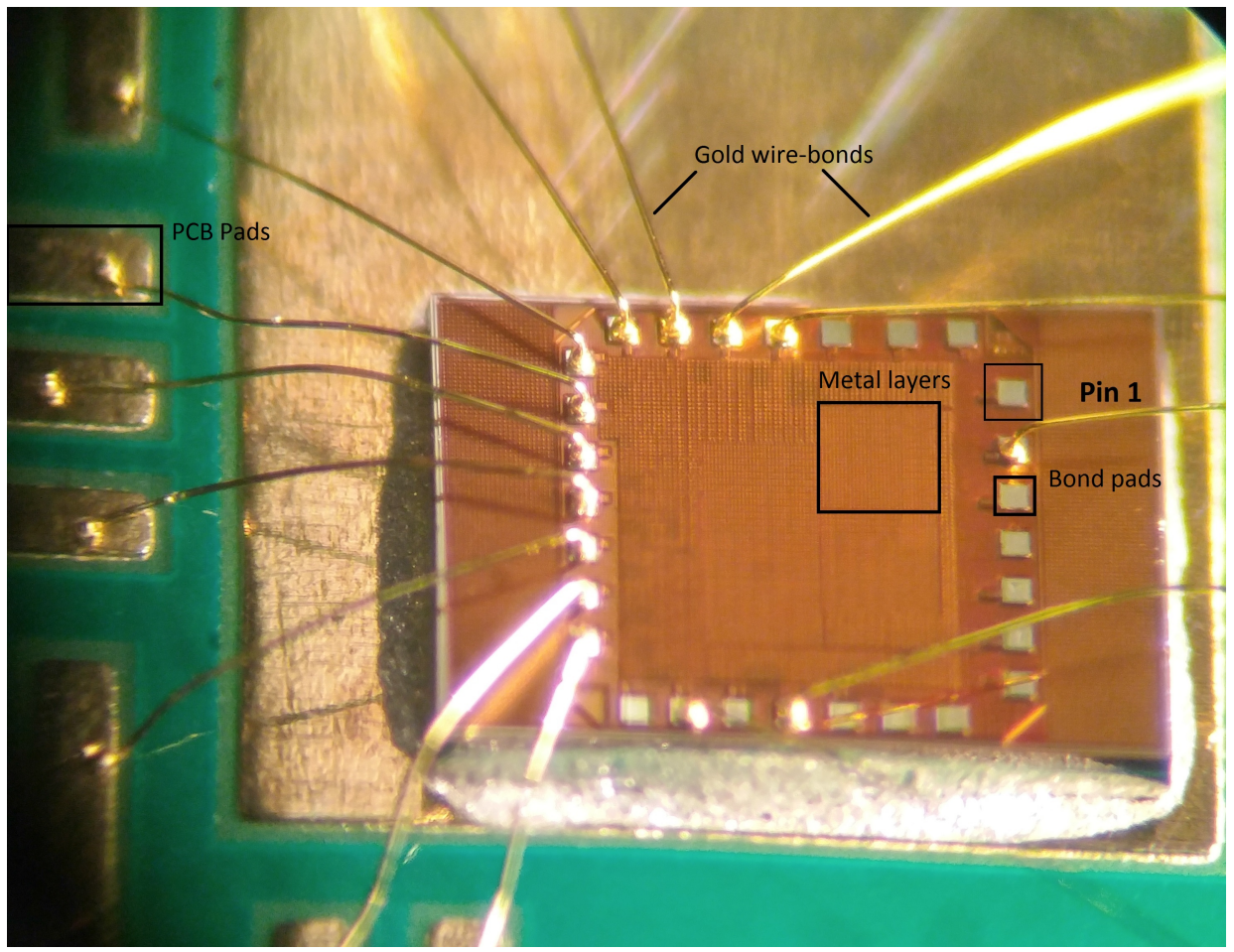

Figure 4.1: In the figure, ICGCUAUM chip is seen glued to a generic PCB and bonded to it with gold bond-wires establishing an electrical connection between the bond-pads of the chip and PCB pads. Twenty eight bond-pads run across the border of the chip and are connected to pads present on the PCB. Sections of metals seen in the pic correspond to metal fills in the chip. The chip dimensions are $1.5 \mathrm{~mm} \times 1.5 \mathrm{~mm}$. 
The chip seen in the figure 4.1 is obtained from CMC and bonded to a generic PCB at Carleton University. Number of bond-pads are seen connected to the pads on PCB by gold bond-wires. These pads on PCB are connected to other pads which are accessible for testing.

The pin diagram for the chip is presented in the figure 4.2. In the figure 4.2 , the

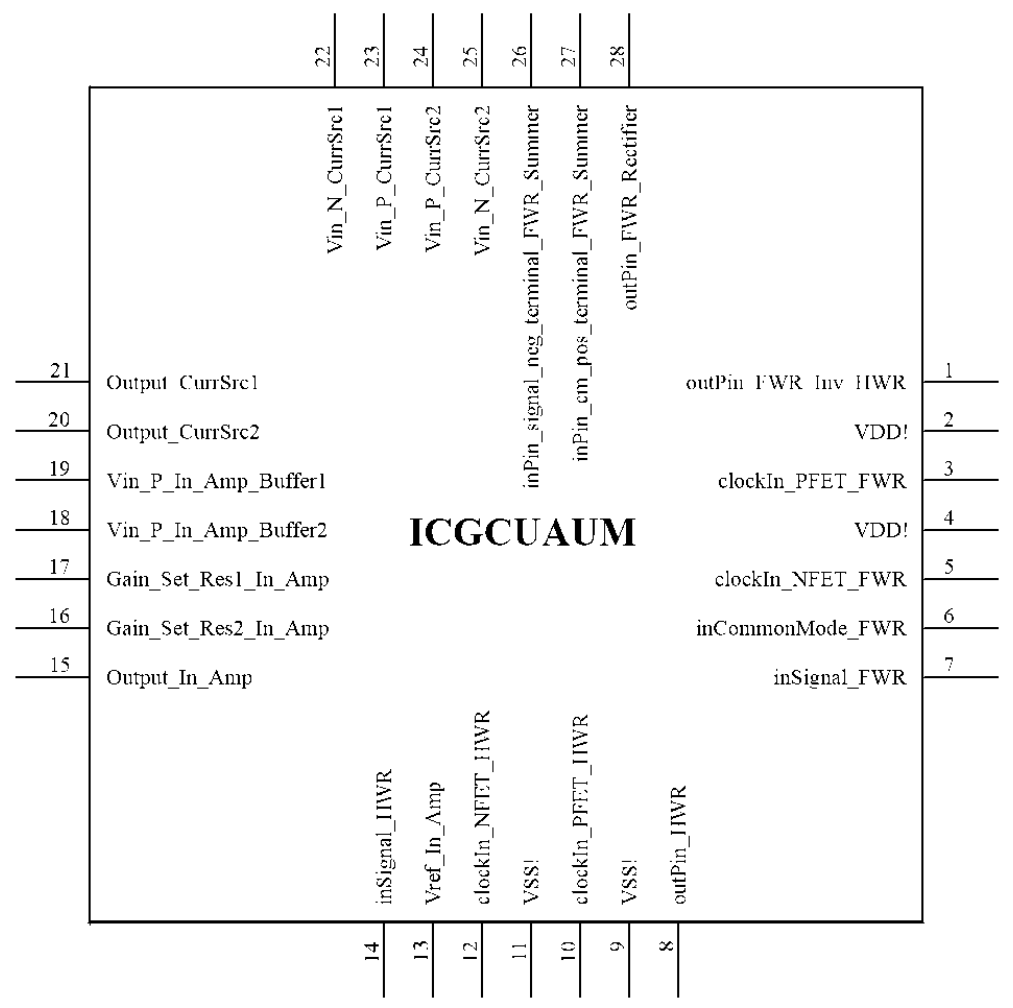

Figure 4.2: The figure shown numbers the pins that correspond to bond-pads on the chip.

pins of the chip are numbered and named intelligibly to recognize the connection and the sub-circuit the pin belongs to. These pins correspond to bond-pads on the chip. The chip is $1.5 \mathrm{~mm} \times 1.5 \mathrm{~mm}$ in dimensions and is not packaged.

\subsubsection{ESD Caution}

Care was taken while handling the chip to ensure the chips were ESD safe. Grounded ESD straps were worn during the testing and tests were conducted 
in an ESD safe environment. Input signals applied were no more than the value of the positive power supply and also the maximum power supply was limited to $1.8 \mathrm{~V}$ as per the technology datasheet.

\subsubsection{Testing Equipment}

The following equipment was used in testing the chip.

- Keithley 6487 Pico-ammeter/ Voltage Source - Used as a voltage source to power up the charge, and in ammeter mode it is used measure the current consumption of the chip. The current consumed aids in calculating the power consumption of the circuit.

- Keithly 2636 System Source Meter - This is another voltage source from Tektronix used to supply power to the chip.

- Agilent 81150A Pulse Function Arbitrary Generator - This equipment from Agilent is a signal generator used to generate sinusoidal and square-wave voltage signals.

- Agilent Technologies DSO5054A Oscilloscope - This equipment is a 4-channel, $500 \mathrm{MHz}$ oscilloscope used to acquire signals and display them.

- Fluke Digital Multimeter - The multimeter is used to measure DC voltages, examine conductivity and for other purposes.

- Breadboard, wires, etc.

- Soldering station and equipment 


\subsubsection{Testing Methodology}

The sequence of tests adopted in testing the chip is:

a. Cold resistance test

b. Testing for unintended connections between pins

c. Unity gain buffer test

d. Module verification testing

e. System verification testing

\subsubsection{Test Results and Debugging}

This section provides information about the tests, their results and discusses the issues the lead to the failure of the chip. Five chips were wire-bonded to a PCB board and subjected to the bring-up and functionality tests.

\section{a. Cold Resistance Test}

Performed before supplying power to the chip, cold resistance test checks for unintended connection between the power rails of the chip and measures the impedance between the positive power supply net and the negative power supply. Generally, a very high impedance between the power rails of the chip is desired.

Using a multimeter, the resistance between positive power supply pin and ground pin of the chip is measured between pin 2 and 11 of the chip. The resistance between the chip is found to be $90 \mathrm{~K} \Omega$.

When a positive voltage of $1.5 \mathrm{~V}$ is supplied to the chip, using the Keithley $6487 \mathrm{pi}$ coammeter the current is measured to be $1.10 \mathrm{~mA}$. Therefore, equivalent resistance between the power rails of the chip is

$$
\text { Equivalent resistance }=\frac{1.5 \mathrm{~V}}{1.1 \mathrm{~mA}}=1.36 \mathrm{~K} \Omega
$$


The equivalent resistance is low because of the bias resistor in the operational amplifiers. The $\sim 12.5 \mathrm{~K} \Omega$ bias resistor in all the operational amplifiers are parallel with one another ensuing in a reduced power rail resistance.

\section{b. Testing for Unintended Connections}

Before supplying power to the chip, any unintended connection between different pins of the chip is tested for using a digital multi-meter.

The DUT's tested did not report any unintended connections between their pins.

\section{c. Unity Gain Buffer Test}

The chip designed comprises of individual operational amplifiers that were intended to be used for the mirrored modified Howland current source. These operational amplifiers were configured as a unity gain operational amplifier to assess the functionality of the fabricated operational amplifier.

When an input signal with both AC and DC components is applied to the noninverting terminal of the unity gain operational amplifier, the virtual ground between the non-inverting terminal and the inverting terminal of the operational amplifier causes the output to follow the input. The load in the circuit is usually a capacitor, but the final buffer stage of the designed op-amp enables the op-amp to support resistive loads.

When a signal of $200 \mathrm{mV}$ amplitude at a $750 \mathrm{mV}$ DC bias is applied at the noninverting terminal of the unity gain buffer, an output identical to input signal is expected at the output. 


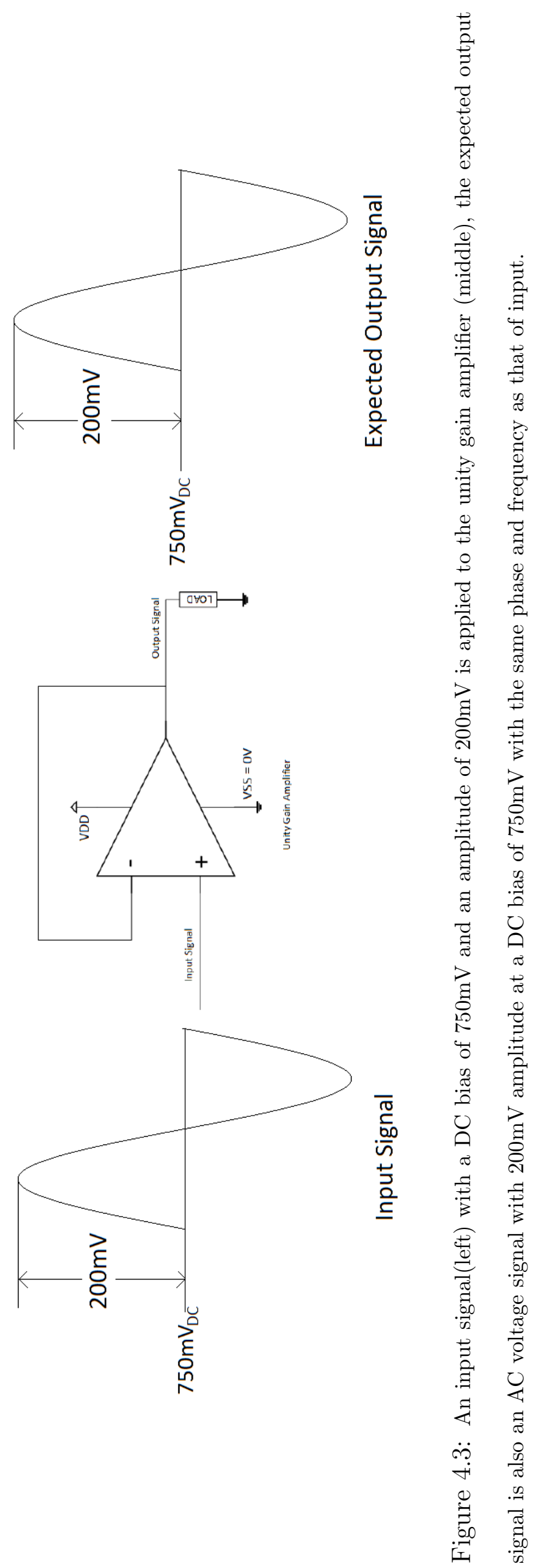


An available fabricated operational amplifier in the ICGCUAUM chip is configured as a buffer that is shown in the figure 4.4 .

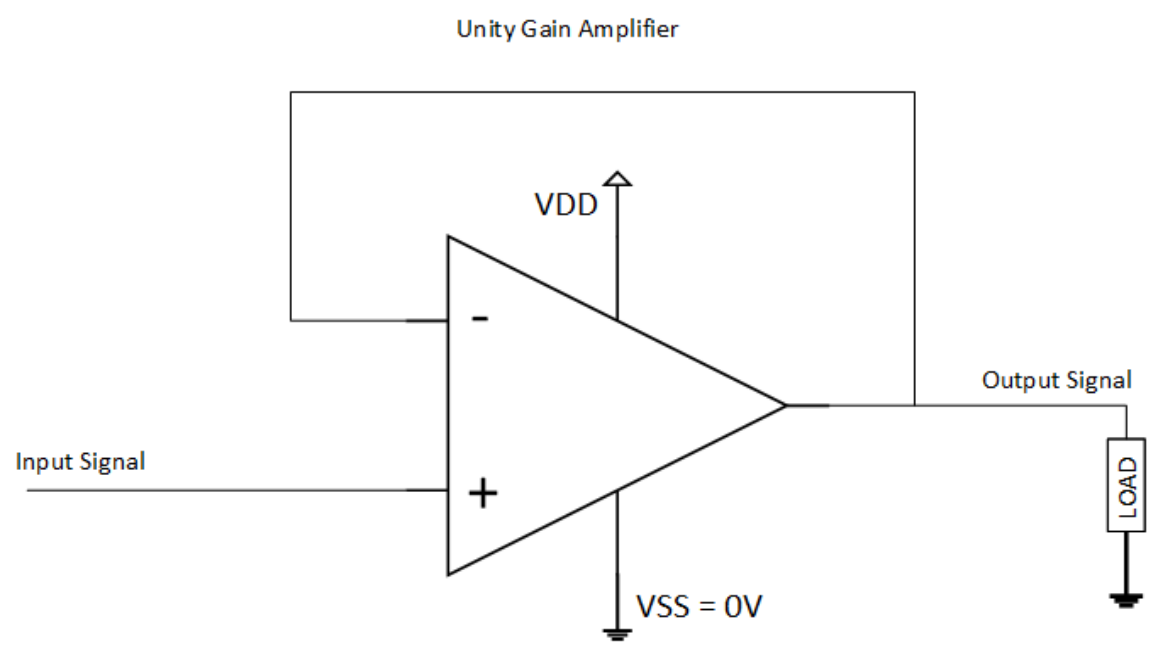

Figure 4.4: An operational amplifier is configured as a buffer shown in the figure.

An AC voltage signal of amplitude $200 \mathrm{mV}$ at a DC bias of $750 \mathrm{mV}$ is applied to the non-inverting terminal of the operational amplifier, which is pins 23 of the chip. The resultant signal at the output of the operational amplifiers is seen in the figure 4.5 . 


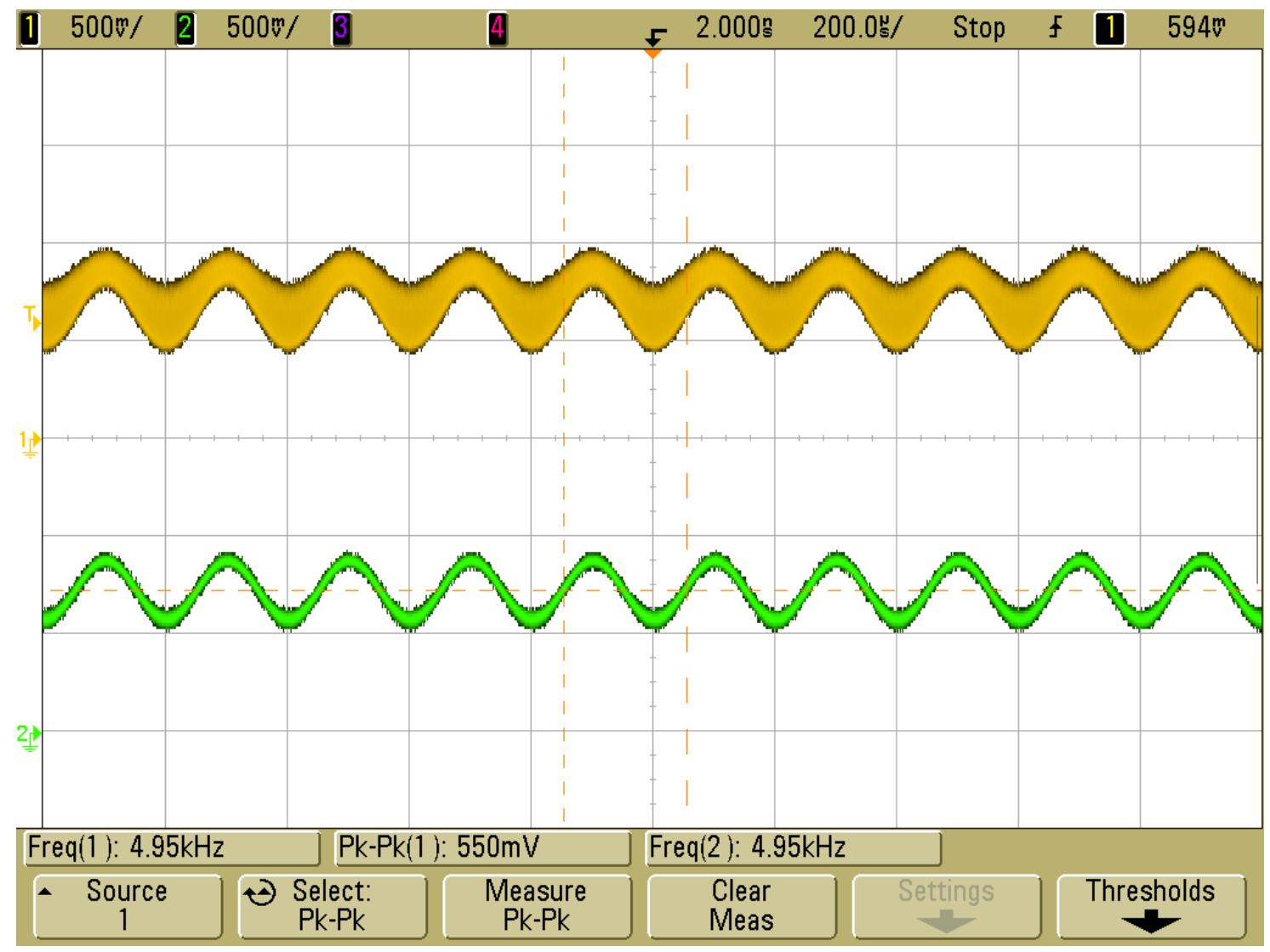

Figure 4.5:

(This figure which has the input and output signals is a screen-shot of the agilent oscilloscope.)In the figure, the input signal(below - green) is a $200 \mathrm{mV}$ amplitude $\mathrm{AC}$ voltage signal at a common mode $\mathrm{DC}$ bias of $750 \mathrm{mV}$. The frequency of the input signal is $5 \mathrm{kHz}$. The output signal(top - yellow) is observed to be a $550 \mathrm{mV}$ peak-to-peak voltage signal at $5 \mathrm{kHz}$. The output signal is seen to oscillate at a frequency of $\sim 23.25 \mathrm{MHz}$. 
The output signal obtained at the output pin of the operational amplifier, which is pin 21, is seen in the figure 4.5. Clearly, the output signal is not identical to the input signal and the operational amplifier is not working as the buffer. An input of $400 \mathrm{mV}$ peak-to-peak voltage is producing an oscillating output with peakto-peak voltage equal to $550 \mathrm{mV}$. The output signal comprises of $5 \mathrm{kHz}$ component and the oscillating frequency component.

The increase in peak-to-peak voltage is due to the oscillation in the output and the frequency of oscillation is $23.2 \mathrm{MHz}$.

The oscillation in the output is obtained by simply biasing the non-inverting input terminal of the operational amplifier at a $\mathrm{DC}$ of $750 \mathrm{mV}$.

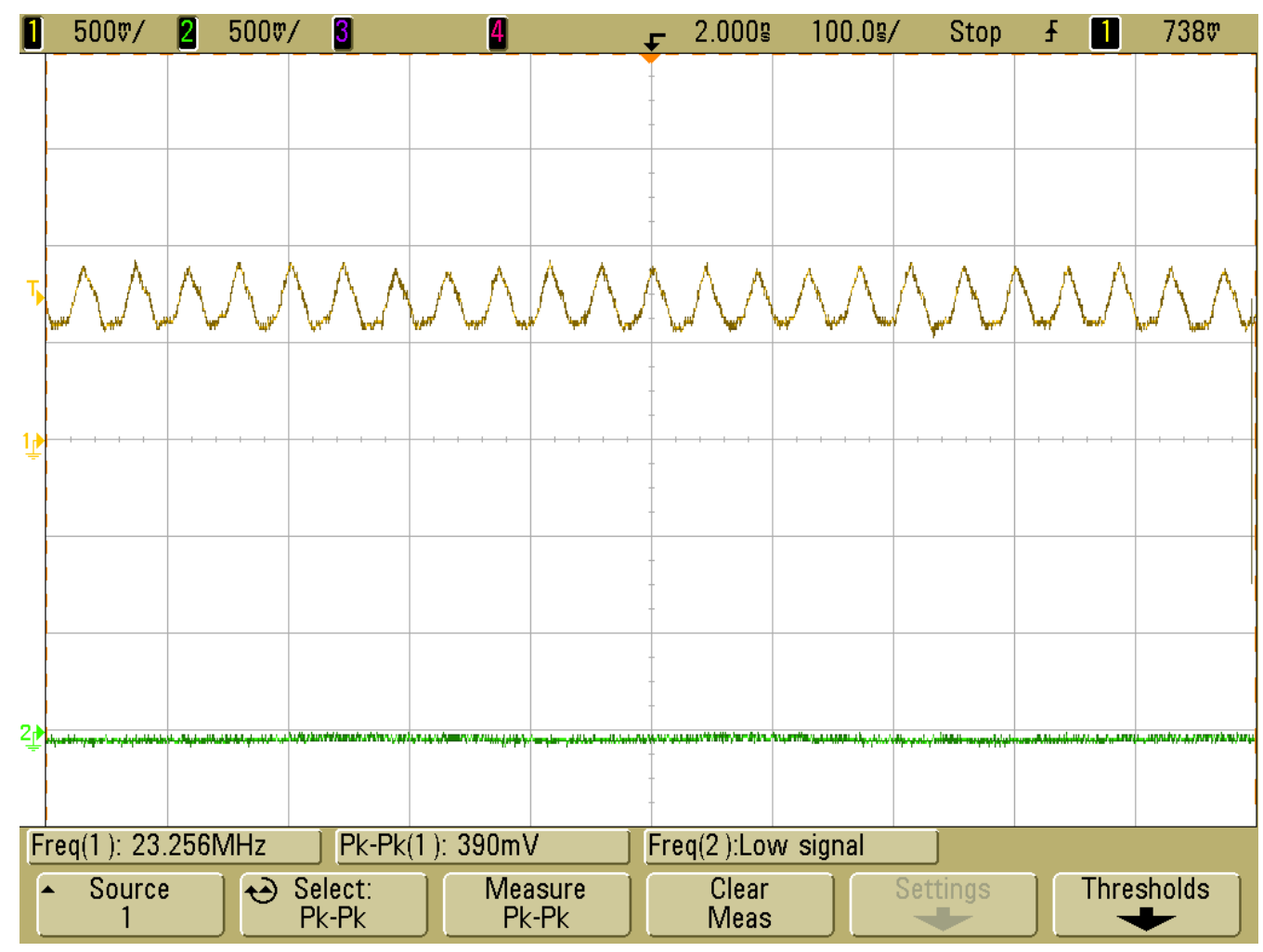

Figure 4.6:

In the figure, the output signal(yellow) is the oscillating output signal produced by the buffer circuit when applied with a DC bias input of $750 \mathrm{mV}$ at its non-inverting input terminal. 
The output signal oscillates at a frequency $23.2 \mathrm{MHz}$ with a peak-to-peak voltage of $390 \mathrm{mV}$ at a DC offset of $750 \mathrm{mV}$, same as the DC input signal, from the ground, which is $0 \mathrm{~V}$. 


\subsection{Failure Analysis}

This section deals with the design issues that lead to the failure of the chip.

The oscillating output at the output pin of the op-amp, unfortunately, marked the end of testing. Unfortunately, internal connections and inaccessible nodes in the op-amp based instrumentation amplifier and the rectifier circuits marked the end of testing and failure of the chip.

The oscillation observed at the output of the op-amp is because of the op-amp's instability due to lack of phase margin and improperly biased load in the buffer stage of the operational amplifier.

\subsubsection{Design Issue I - Phase Margin}

Mathematically speaking, phase margin is the difference between the op-amp's phase shift and $-180^{\circ}$ at the unity-gain frequency. Conceptually, an operational amplifier is unstable if the gain of the circuit is greater than 1 when the phase is $-180^{\circ}$.

The figure 4.7 shows the result of AC simulation of the operational amplifier. The $\mathrm{AC}$ input to the operational amplifier is $1 \mathrm{mV}$ and the $\mathrm{DC}$ bias of the signal is $750 \mathrm{mV}$. The gain and phase plots seen in the figure show that the positive gain of $15.8 \mathrm{~dB}$ at the phase of $-180^{\circ}$ is the reason that the operational amplifier is unstable. 


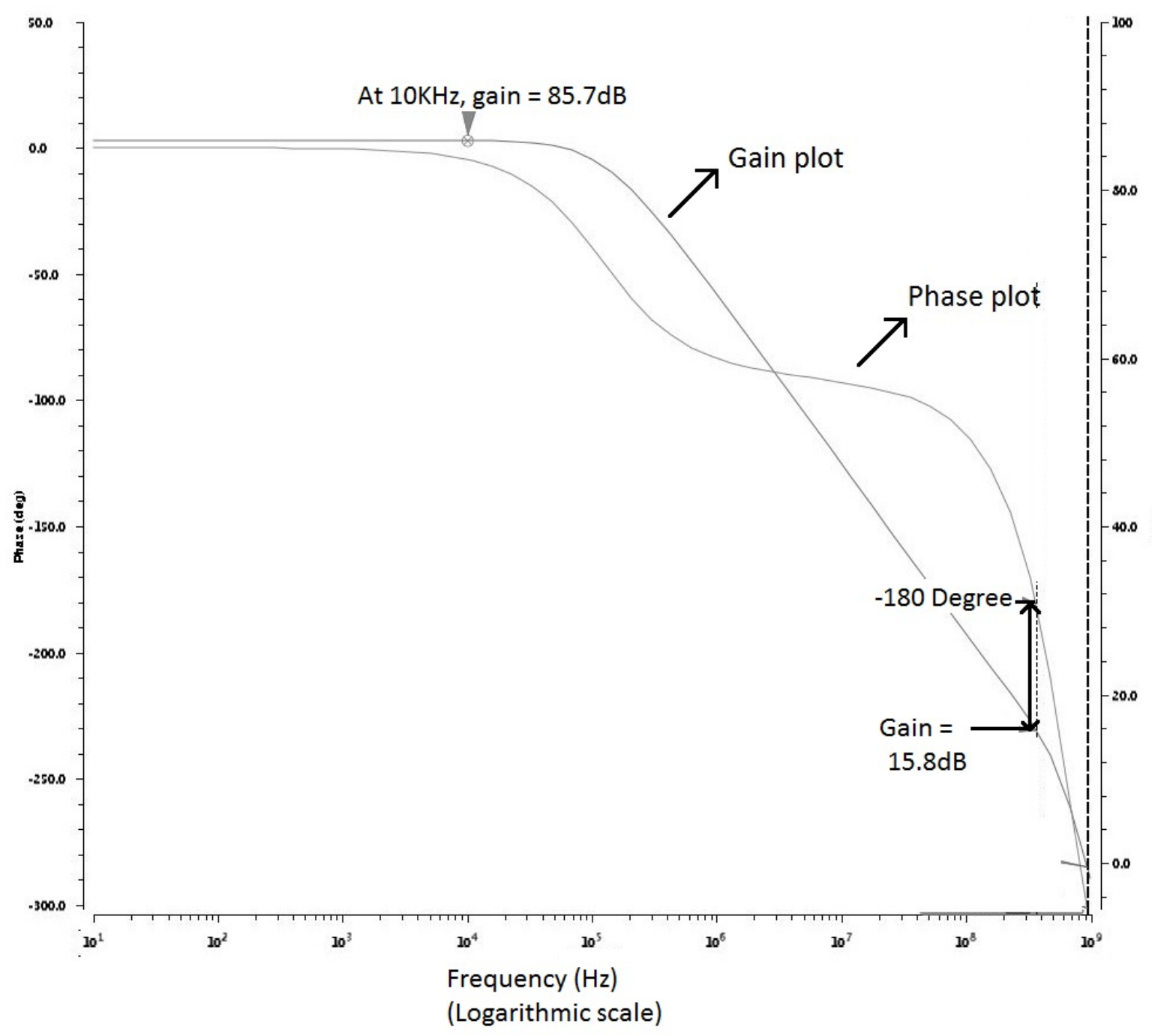

Figure 4.7:

The figure above shows the gain and phase plots of the operational amplifier.

The gain and phase are found for differential inputs of $1 \mathrm{mV}$ peak amplitude at a common mode DC bias of $750 \mathrm{mV}$. The gain at $-180^{\circ}$ phase is $15.9 \mathrm{~dB}$ and the phase margin is $-121^{\circ}$. 
The positive gain at the frequency where the phase is -180 degrees is the cause of oscillations leading to instability.

\subsubsection{Design Issue II - Output Stage}

Typically, the output stage of an op-amp is a source follower and is used to adjust the output DC voltage, increase the current drive of the operational amplifier and also reduce the output impedance. Typically, the output source follower stage is a common drain stage with an active load.

In the op-amp the output of the 2nd differential stage is connected to the gate of the NMOS of the buffer stage which usually is the active load for the NMOS common drain stage, output buffer. 

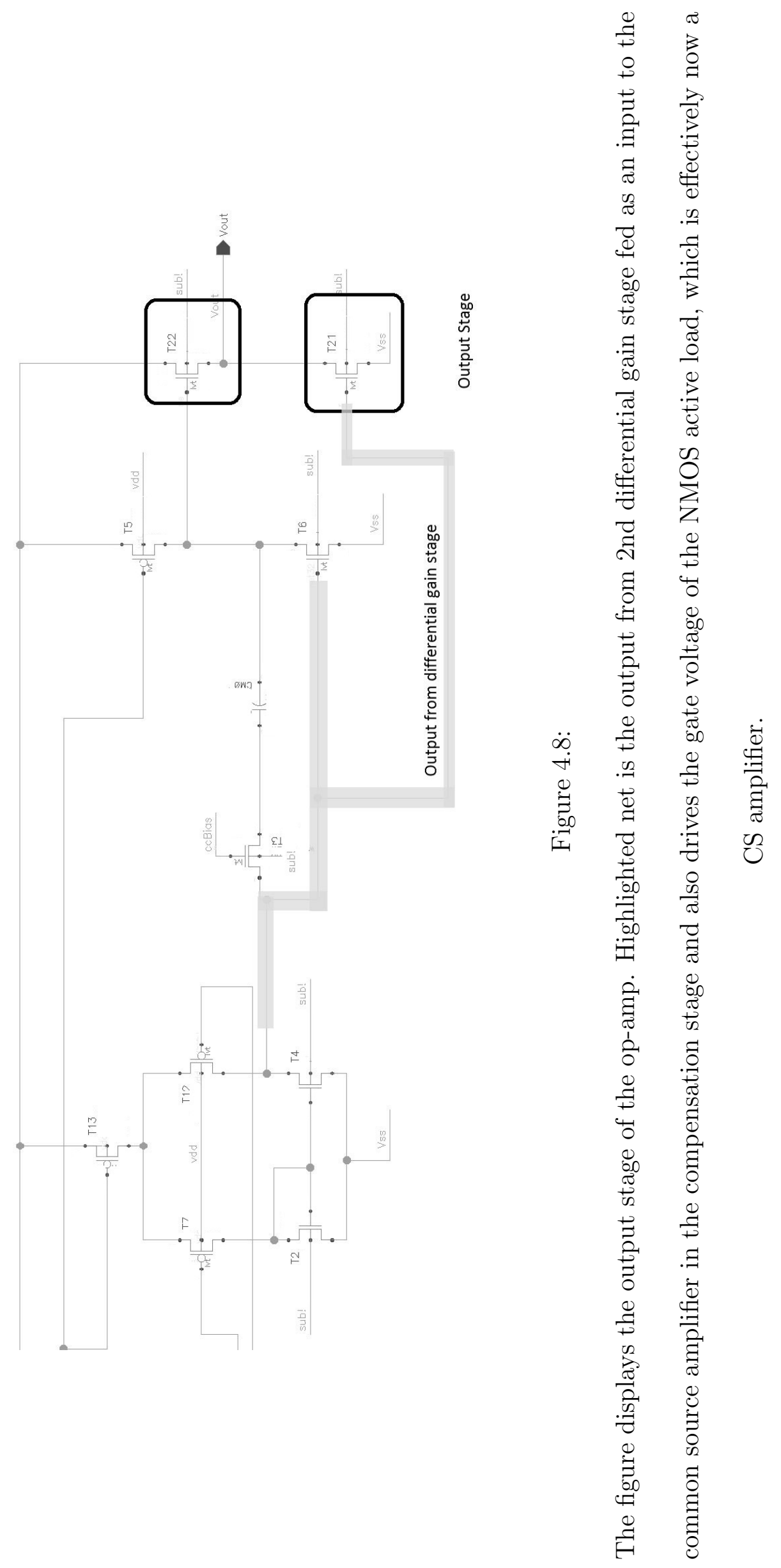
An input signal of considerable small signal magnitude is amplified by the two gain stages and the output of the gain stage when connected to the gate of the NMOS transistor load of the buffer stage, the operating point of the transistor is no longer constant, causing sizeable changes in the current driven in the stage. However, for an input of very small magnitude, the NMOS load transistor operates close to the operating point causing no considerable changes at the output. Besides the two main design issues that were discusses, though not severe but worth mentioning is the absence of on-chip power supply decoupling capacitors. Noise on the power supply rail of the chip due to external interferences like $60 \mathrm{~Hz}$ power line interference, poorly designed voltage source, or faulty breadboards, can cause variations in the bias of the transistors in the circuit. This change in bias results in undesirable outputs. Though it is always advisable to have on-chip decoupling capacitors, this issue was quickly overcome by placing a $10 \mu \mathrm{F}$ tantalum capacitor and $0.1 \mu \mathrm{F}$ ceramic capacitor between the power supply rails on the PCB. 


\section{Chapter 5}

\section{Operational Amplifier}

\section{Re-designed}

This Chapter shows the schematic of a newly designed operational which addresses the issues of the original operational amplifier circuit. AC simulation results and which illustrate the phase and gain of the operational amplfier is shown to testify stability of the new op-amp and a summary of the the specifications is provided at the end of the Chapter.

Reiterating on the issues of the operational amplifier that is fabricated, they are i. Instability due to positive gain at $-180^{\circ}$ phase shift of the output signal.

ii. Improper biasing of the buffer stage load.

\subsection{Circuit Description}

The topology of the operational amplifier remains the same indicating that the working of the circuit remains the same, saving a few changes to the values of the parameters of the components. 
In the figure 5.1, the PMOS transistors T20 and T13 act as current biasing

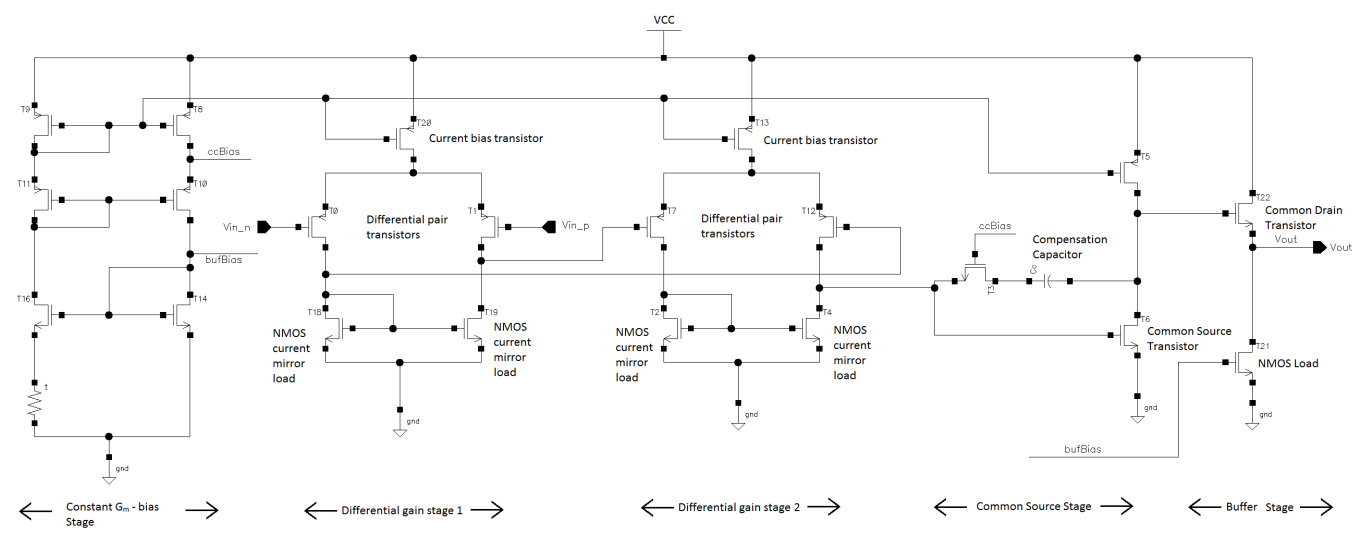

Figure 5.1: The figure illustrates the new operational amplifier schematic that is designed to address the issues that caused the failure of the original operational amplifier. Different stages of the operational amplifier are indicated. The topology of the operational amplifier is the same as the original op-amp and the noticeable change in the schematic is the biasing of the NMOS load of the buffer stage. The NMOS transistor load, T21, is biased by connecting its gate and the drain of the T14 transistor.

transistors for the stages 1 and 2, which are in turn biased by the gate-source voltage of $\mathrm{T} 8$ transistor of the constant $g_{m}$ bias stage.

Current mirror pairs, $(\mathrm{T} 18, \mathrm{~T} 19)$ and $(\mathrm{T} 2, \mathrm{~T} 4)$, are current mirror loads of the differential gain stages 1 and 2 respectively. Differential input transistors, (T0,T1) and $(\mathrm{T} 7, \mathrm{~T} 12)$, are differential pair transistors for the differential gain stages 1 and 2 respectively. The differential pair transistors act as common source amplifiers amplifying the input signal and producing the output that is acquired at the drain of T12 transistor. The output at the drain of T12 transistor is fed to the common source amplifier.

The common source amplifier comprises of T6 transistor that acts as common source transistor and T5 as a load for the stage. The capacitor, $C_{c}$, and transistor 
T3 compensate the op-amp and makes it stable.

The transistors T22 and T21 constitute the final buffer stage of the operational amplifier.

The table 5.1 shows the parameters pertaining to the components in the schematic and their values.

The significant change in the circuit compared to the original operational amplfier is the change in the value of the compensation capacitor, the triode transistor and the final buffer stage of the circuit. 


\begin{tabular}{|c|c|c|}
\hline Component & Type & Value \\
\hline T0 & PMOS & $\mathrm{W}=10 \mu \mathrm{m} ; \mathrm{L}=480 \mathrm{~nm}$ \\
\hline $\mathrm{T} 1$ & PMOS & $\mathrm{W}=10 \mu \mathrm{m} ; \mathrm{L}=480 \mathrm{~nm}$ \\
\hline $\mathrm{T} 7$ & PMOS & $\mathrm{W}=10 \mu \mathrm{m} ; \mathrm{L}=480 \mathrm{~nm}$ \\
\hline $\mathrm{T} 12$ & PMOS & $\mathrm{W}=10 \mu \mathrm{m} ; \mathrm{L}=480 \mathrm{~nm}$ \\
\hline $\mathrm{T} 2$ & NMOS & $\mathrm{W}=960 \mathrm{~nm} ; \mathrm{L}=360 \mathrm{~nm}$ \\
\hline $\mathrm{T} 4$ & NMOS & $\mathrm{W}=960 \mathrm{~nm} ; \mathrm{L}=360 \mathrm{~nm}$ \\
\hline $\mathrm{T} 18$ & NMOS & $\mathrm{W}=960 \mathrm{~nm} ; \mathrm{L}=360 \mathrm{~nm}$ \\
\hline T19 & NMOS & $\mathrm{W}=960 \mathrm{~nm} ; \mathrm{L}=360 \mathrm{~nm}$ \\
\hline T6 & NMOS & $\mathrm{W}=5 \mu \mathrm{m} ; \mathrm{L}=480 \mathrm{~nm}$ \\
\hline T5 & PMOS & $\mathrm{W}=15 \mu \mathrm{m} ; \mathrm{L}=360 \mathrm{~nm}$ \\
\hline $\mathrm{T} 20$ & PMOS & $\mathrm{W}=5 \mu \mathrm{m} ; \mathrm{L}=360 \mathrm{~nm}$ \\
\hline $\mathrm{T} 13$ & PMOS & $\mathrm{W}=5 \mu \mathrm{m} ; \mathrm{L}=360 \mathrm{~nm}$ \\
\hline $\mathrm{T} 22$ & NMOS & $\mathrm{W}=9 \mu \mathrm{m} ; \mathrm{L}=360 \mathrm{~nm}$ \\
\hline $\mathrm{T} 21$ & NMOS & $\mathrm{W}=14 \mu \mathrm{m} ; \mathrm{L}=360 \mathrm{~nm}$ \\
\hline $\mathrm{T} 3$ & NMOS & $\mathrm{W}=1.8 \mu \mathrm{m} ; \mathrm{L}=2.4 \mu \mathrm{m}$ \\
\hline $\mathrm{T} 14$ & NMOS & $\mathrm{W}=720 \mathrm{~nm} ; \mathrm{L}=360 \mathrm{~nm}$ \\
\hline $\mathrm{T} 16$ & NMOS & $\mathrm{W}=2 \mu \mathrm{m} ; \mathrm{L}=360 \mathrm{~nm}$ \\
\hline $\mathrm{T} 8$ & PMOS & $\mathrm{W}=2.68 \mu \mathrm{m} ; \mathrm{L}=360 \mathrm{~nm}$ \\
\hline T9 & PMOS & $\mathrm{W}=2.68 \mu \mathrm{m} ; \mathrm{L}=360 \mathrm{~nm}$ \\
\hline $\mathrm{T} 11$ & PMOS & $\mathrm{W}=360 \mathrm{~nm} ; \mathrm{L}=360 \mathrm{~nm}$ \\
\hline $\mathrm{T} 10$ & PMOS & $\mathrm{W}=360 \mathrm{~nm} ; \mathrm{L}=360 \mathrm{~nm}$ \\
\hline Bias resistor & - & $12.5 \mathrm{~K} \Omega$ \\
\hline$C_{c}$ & - & $1 \mathrm{pF}$ \\
\hline
\end{tabular}

Table 5.1: New Operational amplifier components and their values 


\subsection{Simulations}

This section illustrates the simulation results of AC and transient analysis.

The AC analysis shows that the new operational amplifier is stable with adequate phase margin and also the negative gain at $-180^{\circ}$ phase shift of the output. In

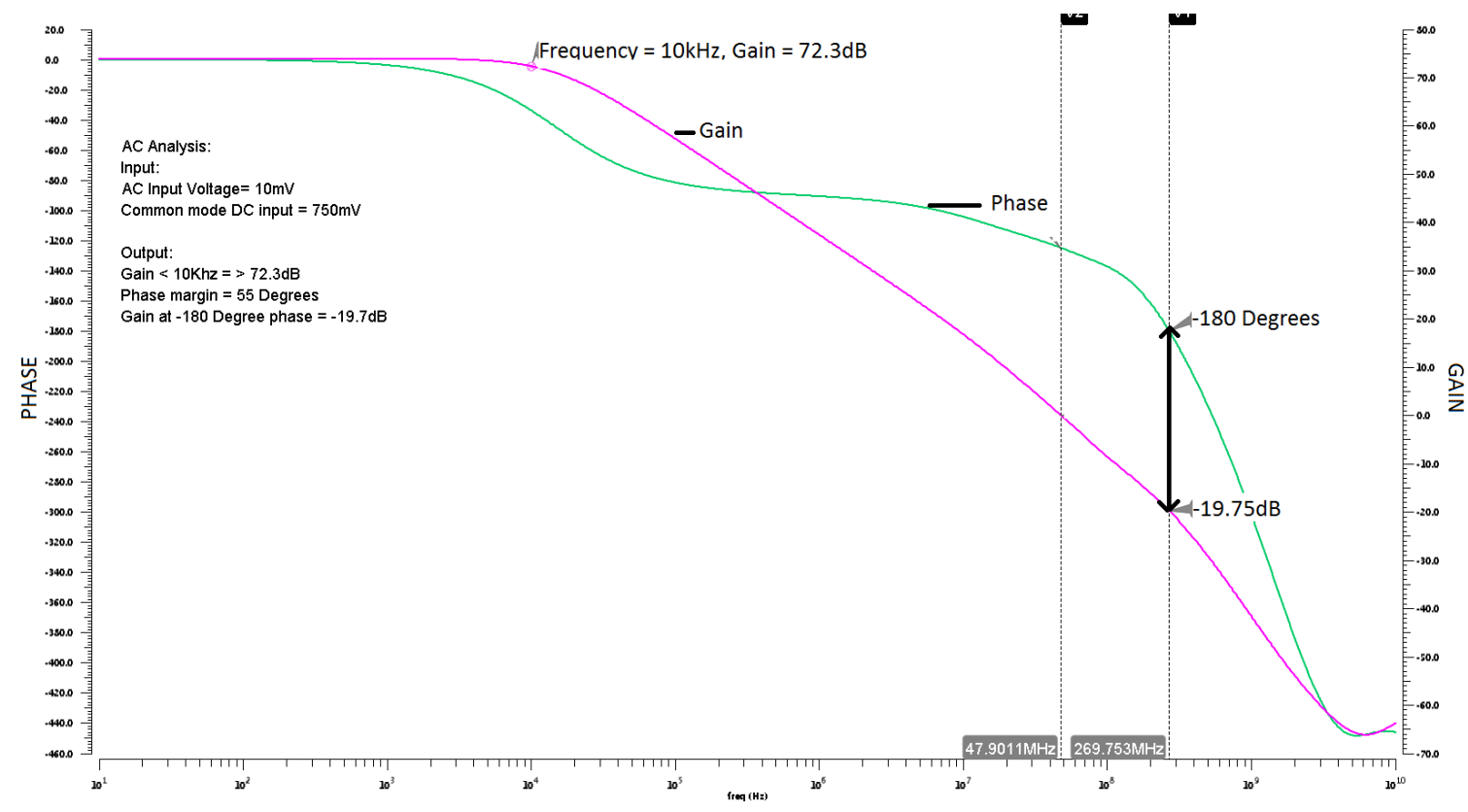

Figure 5.2: In the figure, the phase (pink) and gain (green) plots show that the phase margin and gain at $-180^{\circ}$ phase are $55^{\circ}$ and $-19.7 \mathrm{~dB}$. The negative gain at $-180^{\circ}$ phase of the output shows that the operational amplifier is stable.

the figure 5.2, for a differential $\mathrm{AC}$ input of $10 \mathrm{mV}$ AC analysis shows that phase margin is $55^{\circ}$ and the gain at $-180^{\circ}$ is $-19.7 \mathrm{~dB}$. These values show that operational amplifier is stable.

The transient analysis is performed to show the differential gain, common mode rejection and calculate the common mode rejection ratio of the circuit.

Shown in the figure is the output of the operational amplifier with peak-topeak voltage of $8.2 \mathrm{mV}$ and frequency of $10 \mathrm{kHz}$. Differential inputs of peak-topeak amplitude $2 \mathrm{uV}$ at $750 \mathrm{mV}$ common mode $\mathrm{DC}$ bias and frequency of $10 \mathrm{kHz}$ is 

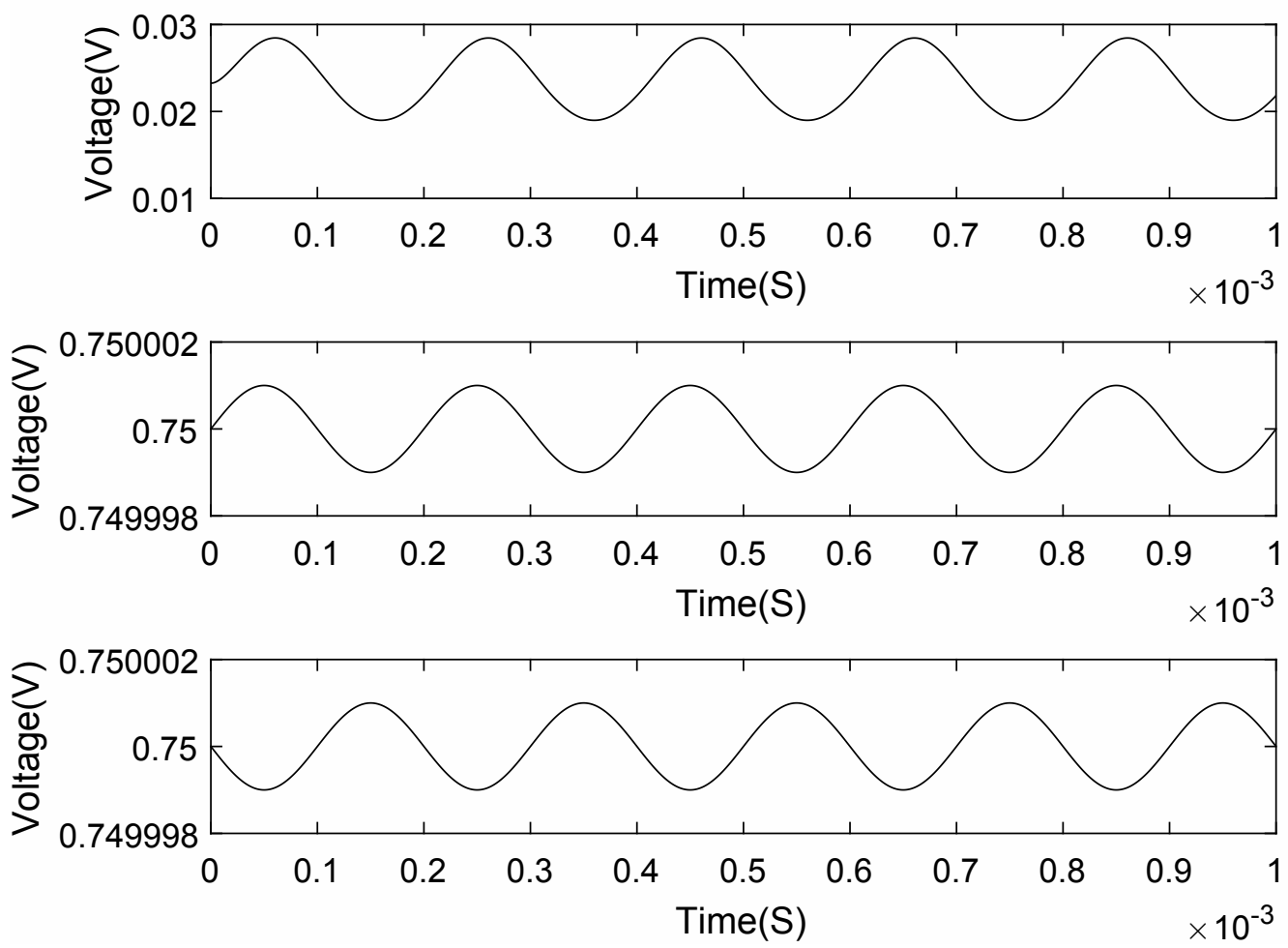

Figure 5.3: From top to bottom: The output voltage signal of the operational amplifier has a peak-to-peak voltage of $8.2 \mathrm{mV}$ and a frequency of $10 \mathrm{kHz}$, differential non-inverting input voltage of peak-to-peak voltage $2 \mathrm{uV}$ at a frequency of $10 \mathrm{kHz}$ and common mode $\mathrm{DC}$ input of $750 \mathrm{mV}$, differential inverting input voltage of peak-to-peak voltage $2 \mathrm{uV}$ at a frequency of $10 \mathrm{kHz}$ and common mode DC input of $750 \mathrm{mV}$

applied to the operational amplifier.

Therefore, the differential gain of the operational amplifier is

$$
A_{D}=20 \log \frac{8.2 m V}{2 u V}=72.3 d B
$$

Now for the common mode rejection, common mode inputs of peak-to-peak amplitude $2 \mathrm{mV}$ at $750 \mathrm{mV}$ common mode DC bias and frequency of $10 \mathrm{kHz}$ is applied to the operational amplifier. The output observed, in figure, is a voltage signal of peak-to-peak voltage $1.6 \mathrm{uV}$. Therefore, the differential gain of the operational 

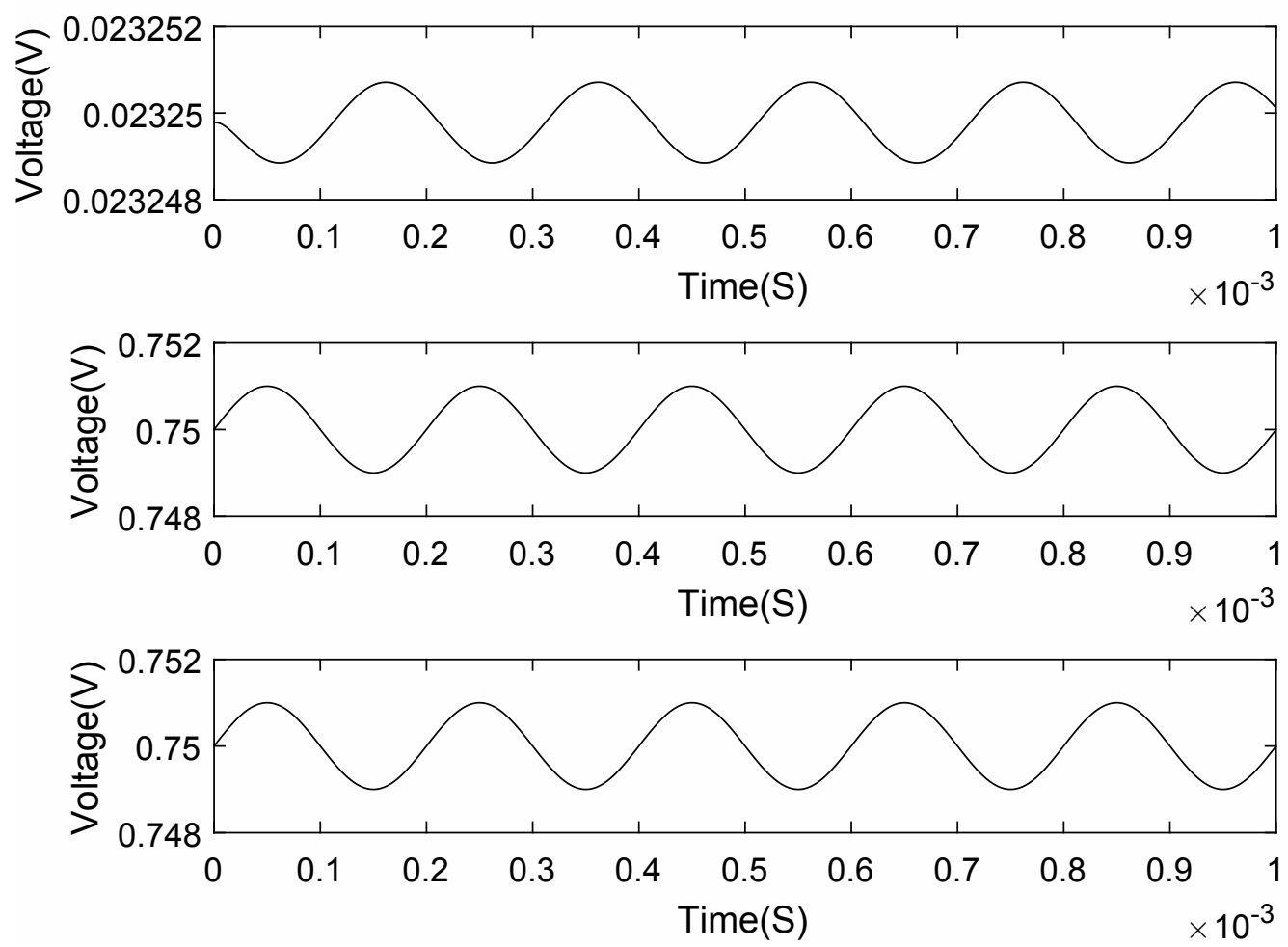

Figure 5.4: From top to bottom: The output voltage signal of the operational amplifier has a peak-to-peak voltage of $1.6 \mathrm{uV}$ and a frequency of $10 \mathrm{kHz}$, common mode non-inverting input voltage of peak-to-peak voltage $2 \mathrm{mV}$ at a frequency of $10 \mathrm{kHz}$ and common mode $\mathrm{DC}$ input of $750 \mathrm{mV}$, common mode inverting input voltage of peak-to-peak voltage $2 \mathrm{mV}$ at a frequency of $10 \mathrm{kHz}$ and common mode DC input of $750 \mathrm{mV}$

amplifier is

$$
A_{C}=20 \log \frac{1.6 u V}{2 m V}=-61.9 d B
$$

Thus, the common mode rejection ratio of the operational amplifier is

$$
C M R R=72.3-(-61.9 d B)=134.2 d B
$$

\subsection{Discussion}

The new operational amplifier that is designed has been an improvement upon the original operational amplifier. The stability of the operational amplifier has 
been achieved by increasing the value of the compensation capacitor. The increased value of the compensation capacitor, $C_{c}$, increased the phase margin and also caused the gain at $-180^{\circ}$ to fall below to the value $-19.2 \mathrm{~dB}$. This is because the increased value of capacitance reduces the pole dominant pole frequency and caused and early drop in gain of the operational amplifier. As a result, the bandwidth has reduced but stability is achieved.

The phase margin of $55^{\circ}$ has not been the result only of the increased value of capacitance but also reduced open loop differential gain. The open loop differential gain of the operational amplifier has been reduced to $72.3 \mathrm{~dB}$ from $85.7 \mathrm{~dB}$. This is a direct result of reduction in gain of the common source amplifier stage and the buffer stage. The reduced (W/L) ratio of the T5 transistor of the CS stage, and the increased $(\mathrm{W} / \mathrm{L})$ ratios of $\mathrm{T} 22$ and $\mathrm{T} 21$ transistors have reduced the gain of the CS stage and buffer stage respectively.

The increase in $(\mathrm{W} / \mathrm{L})$ ratios of the transistors of the buffer stage reduce the output impedance of the operational amplifier to a very low value of $719.1 \Omega$.

Reduced current in the common source stage reduced the power consumption of the operational amplifier when compared to the original operational amplifier. The current consumption of the new op-amp is $105 \mu \mathrm{A}$. Therefore, for a power supply of $1.5 \mathrm{~V}$, the power consumption is $157.5 \mu \mathrm{W}$.

\subsection{Chapter Summary}

The following table 5.2 outlines the specifications of the post-fab new operational amplifier that are determined through simulations. 


\begin{tabular}{|c|c|}
\hline Specification & Value \\
\hline Supply voltage & $1.5 \mathrm{~V}$ \\
\hline Differential gain @ $10 \mathrm{kHz}$ & $72.3 \mathrm{~dB}$ \\
\hline Common mode rejection @ $10 \mathrm{kHz}$ & $-61.3 \mathrm{~dB}$ \\
\hline Common mode rejection ratio & $133.6 \mathrm{~dB}$ \\
\hline UGBW & $47.9 \mathrm{MHz}$ \\
\hline Input common mode range & $\sim(2 \mathrm{mV}, 1 \mathrm{~V})$ \\
\hline Output voltage swing & $\sim(75 \mathrm{mV}, 1 \mathrm{~V})$ \\
\hline Offset voltage & $85 \mu \mathrm{V}$ \\
\hline Output Impedance & $720 \Omega$ \\
\hline Power consumption & $157.5 \mu \mathrm{W}$ \\
\hline
\end{tabular}

Table 5.2: Post-fab new operational amplifier simulated specifications 


\section{Conclusion}

An integrated circuit was designed to determine the bioimpedance of a biological subject. Bioimpedance is determined by injecting an AC current of constant RMS magnitude into the subject and measuring the voltage response of the subject. The current injection circuit, voltage measurement circuit, and post-processing circuit are based on operational amplifiers.

Simulations of the cascaded gain stage operational amplifier predict a high gain of $85.7 \mathrm{~dB}$, common mode gain of $-48.4 \mathrm{~dB}$ and a common mode rejection ratio of 134.1dB. The simulated bandwidth of the operational amplifier is $\sim 100 \mathrm{kHz}$. The simulated DC power consumption of the operational amplifier is $165.1 \mu \mathrm{W}$. Testing the fabricated operational amplifier showed that the operational amplifier oscillated and that was found to be due to inadequate phase margin. The instability in the operational amplifier prompted re-designing it. The new operational amplifier is presented in Chapter 5 .

When simulated, the re-designed operational amplifier had a phase margin of $55^{\circ}$ and a gain margin of $19.75 \mathrm{~dB}$. While the change in design the phase margin of the operational amplifier and stabilizes the circuit, the simulated differential gain reduced to $72.3 \mathrm{~dB}$ at $10 \mathrm{kHz}$ which was $85.7 \mathrm{~dB}$ for the first operational amplifier. The common mode gain for a $10 \mathrm{kHz}$ signal has increased to $\sim 61 \mathrm{~dB}$. Thus, the 
common mode rejection ratio is $133.3 \mathrm{~dB}$.

A Mirrored Modified Howland current source, which is a well-known operational amplifier based voltage controlled current source, was designed to inject current into a biological subject. The current source simulates with different output current for different input volt- age for a range of frequencies. For frequencies ranging from $1 \mathrm{~Hz}$ to $100 \mathrm{kHz}$, currents up to $700 \mu \mathrm{A}$ can be injected. The output impedance of the current source is simulated to be $6.7 \mathrm{MHz}$, which is very high when compared to typical whole body bioimpedance values under normal conditions ranging between $550 \Omega$ to $750 \Omega$.

An instrumentation amplifier is designed to acquire the voltage response of the subject that is produced when current is injected into the subject. When simulated, the instrumentation amplifier that is operated at unity gain shows a common mode rejection ratio of $111.9 \mathrm{~dB}$. The gain of the instrumentation amplifier can be changed by changing the value of its gain setting resistor, RG. This allows for amplifying weak signals. The simulated DC power consumption of the instrumentation amplifier is $477.7 \mu \mathrm{W}$. The output of the instrumentation amplifier is fed as an input to a diode-less rectifier circuit that is designed using transmission gates. The output of the rectifier circuit is a fully rectified voltage signal whose RMS magnitude divided by the RMS magnitude of the injected current provides the estimate of the bioimpedance.

Having summarized the integrated circuit and its sub-circuits, the contribution of the thesis to the research are:

- The diode-less rectifier circuit can rectify a signal with low voltage magnitude and enables low voltage operation. It eliminates the loss in voltage due to the 
threshold voltage of a diode. A typical diode has a threshold voltage of $0.7 \mathrm{~V}$, but the operating voltage amplitudes in the case of bioimpedance are much lower than $0.7 \mathrm{~V}$. However, a pass transmission gate passes the signal without any loss in voltage. A common issue with regards to pass transmission gate is the floating output node. In the case of the full-wave gate rectifier, the common mode DC input of the summation amplifier pulls the transmission gate output node to its common mode DC input voltage. And, when an AC signal is applied to the input of the transmission gate, an output is observed at the output of the summation amplifier. As a possible future work, in the case of a half-wave rectifier, the issue with floating output node can be dealt by having an additional circuit which recognizes the common mode DC in the input signal and applies that to an operational amplifier operating at unity gain whose other input is the output of the transmission gate.

- Another major contribution of this thesis is the topology of the operational amplifier.

The cascaded differential gain stage improves the gain of the operational amplifier. The gain of the differential gain stage designed as a part of the operational amplifier is doubled due to the current mirror load when compared to the differential gain stage with active loads. The gain of the cascaded differential gain stages is increased by a factor of 4 . This increase in differential gain of the operational amplifier increases the common mode rejection ratio.

The precisely designed current mirror load also increases output voltage swing. The research on bioimpedance is growing rapidly due to the applicability of bioimpedance across a wide range of applications, from purely bio-medical systems to commer- 
cial products such as cellular monitoring equipment to body fat scales and other measurement systems.

Several commercial products in the market perform single frequency analysis, but research shows that multi-frequency impedance analysis, BIS, is more accurate. The designed ASIC circuit could address this issue. Also, the number of fully analog ASIC solutions to the problem of finding bioimpedance is small in number. This thesis tries to add to the research and also to the number of ASIC solutions that determine bioimpedance.

Possible future work of this thesis could be:

- Improving the bandwidth of the rectifier circuit.

- The output from the rectifier is a fully-rectified output signal whose RMS magnitude needs to be calculated along with the RMS magnitude of the injected current. In this regards, it is possible to design a digital signal processing block to acquire the analog output from the rectifier and produce the RMS magnitude and/ or bioimpedance value.

- The Monte-Carlo simulation results from the tool seem to be very tight, hence needs more consideration in terms of veracity of the results. 


\section{Appendix A}

\section{Monte Carlo Simulations}

This appendix demonstrates the monte-carlo simulations for differential gain, $A_{D}$, and common mode gain, $A_{C}$, of the fabricated operational amplifier to compute common mode rejection ratio, CMRR.

As stated before, common mode rejection ratio of an operational amplifier, CMRR, is computed by the equation in decibels $(\mathrm{dB})$

$$
C M R R=A_{D}+A_{C}
$$

\section{A.1 Differential Gain}

A differential input of $1 \mathrm{mV}$ amplitude is applied at the non-inverting and inverting terminals of the operational amplifier. For a single supply voltage of $1.5 \mathrm{~V}$ and $0 \mathrm{~V}$ as the ground reference, monte-carlo simulations for differential gain of the operational amplifier is found at frequencies $1 \mathrm{kHz}, 5 \mathrm{kHz}, 10 \mathrm{kHz}, 50 \mathrm{kHz}, 75 \mathrm{kHz}$ and $100 \mathrm{kHz}$.

The following figures illustrate the differential gain at respective frequencies for a 1000-point monte-carlo simulation. 


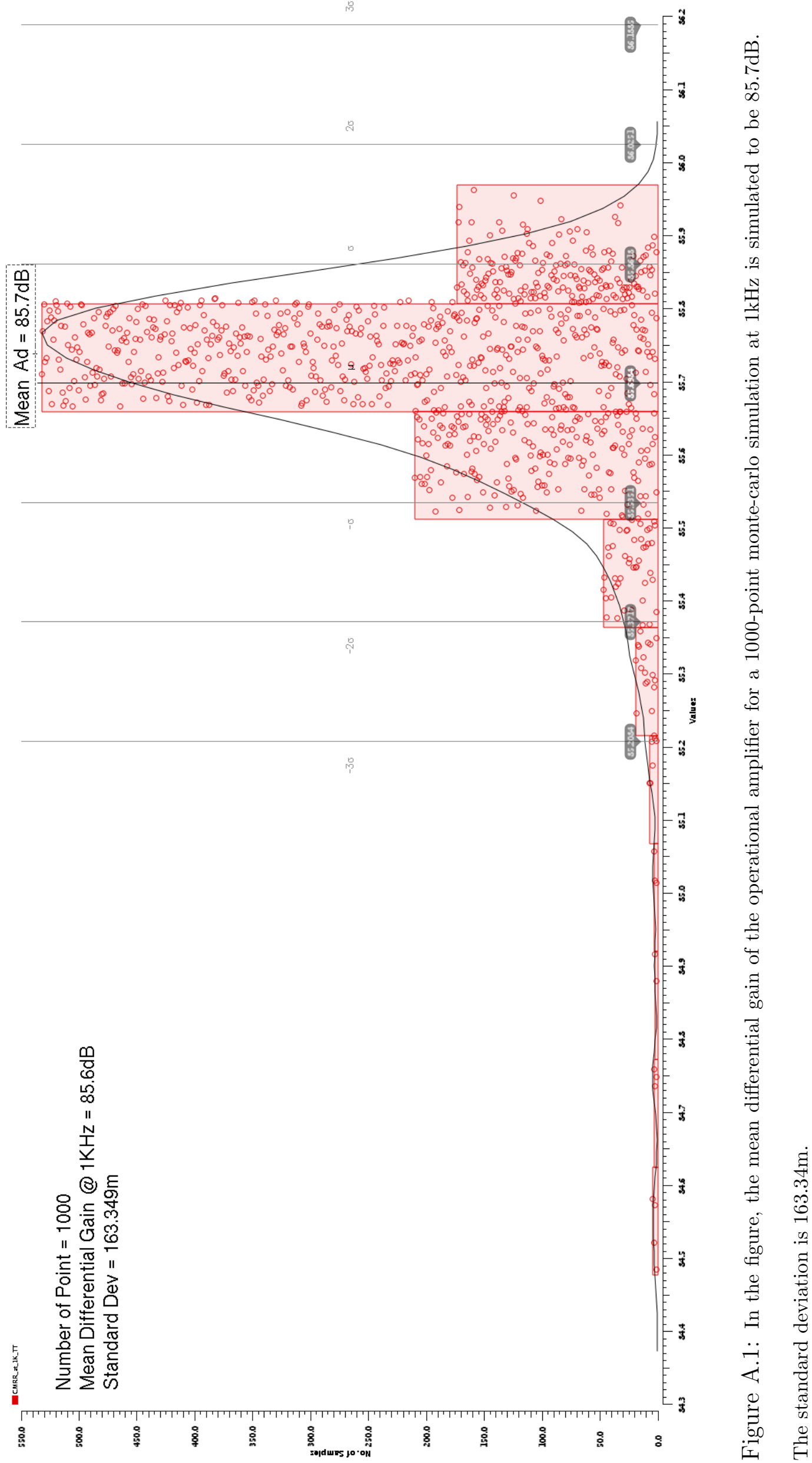




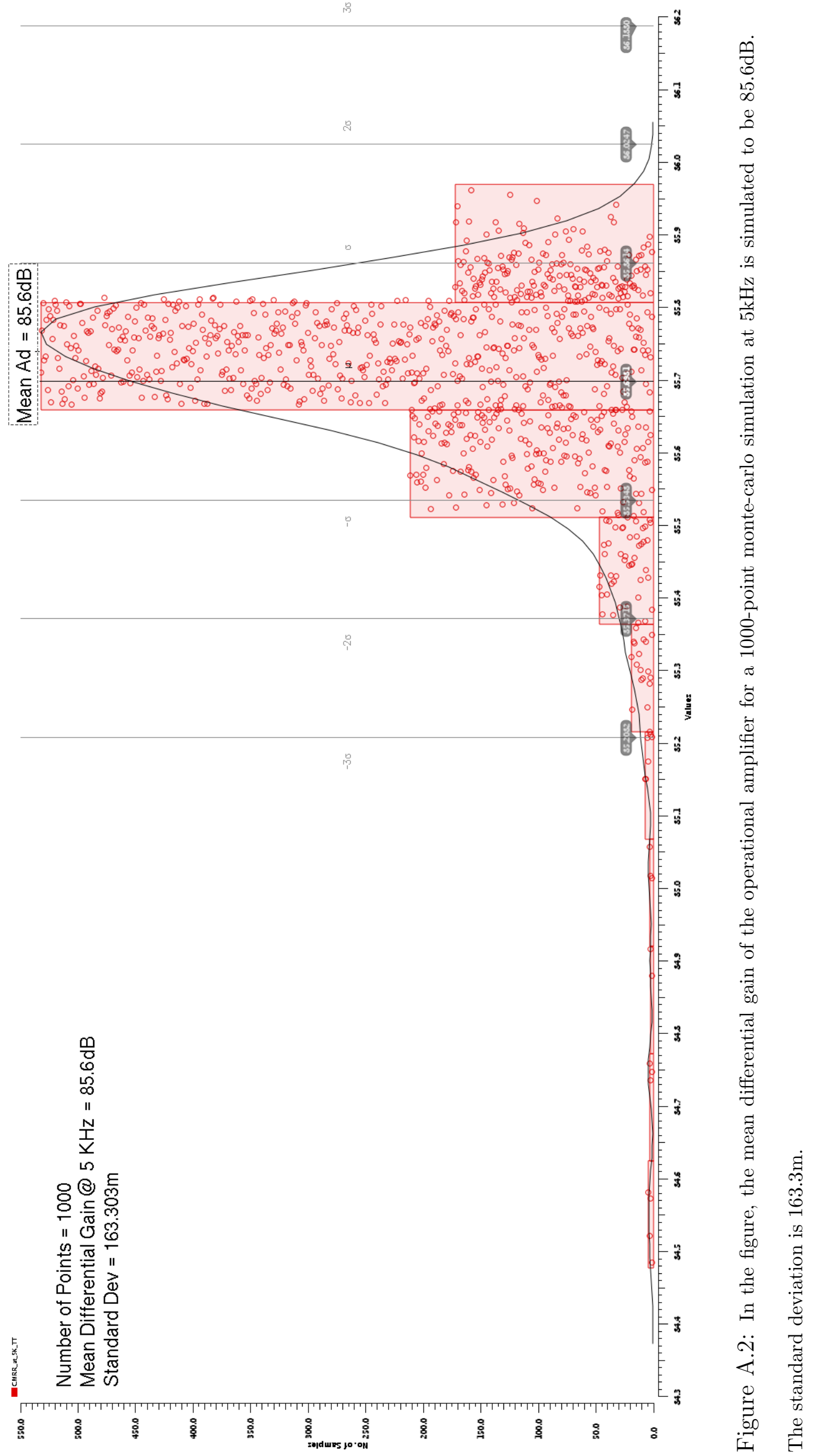




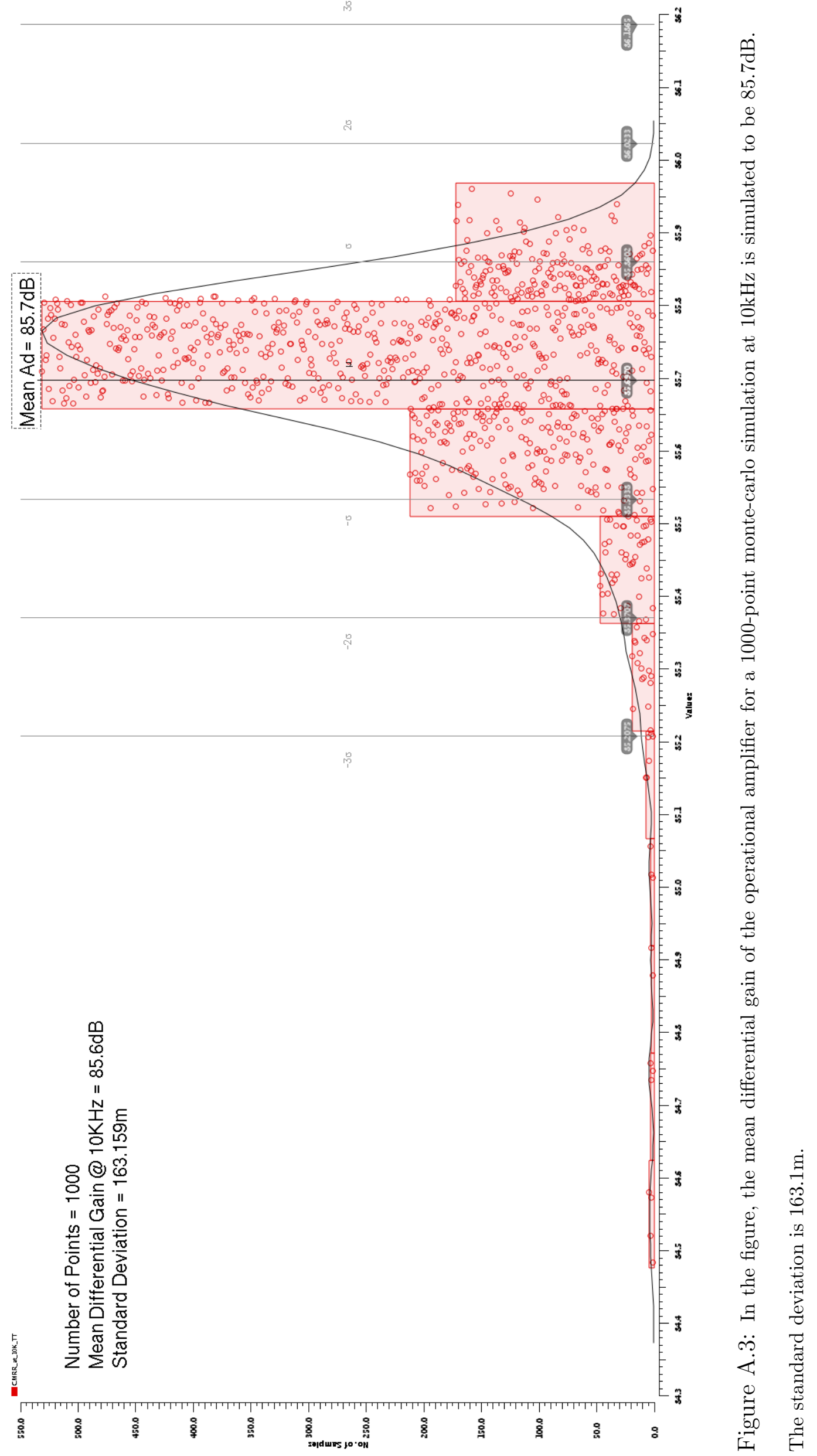




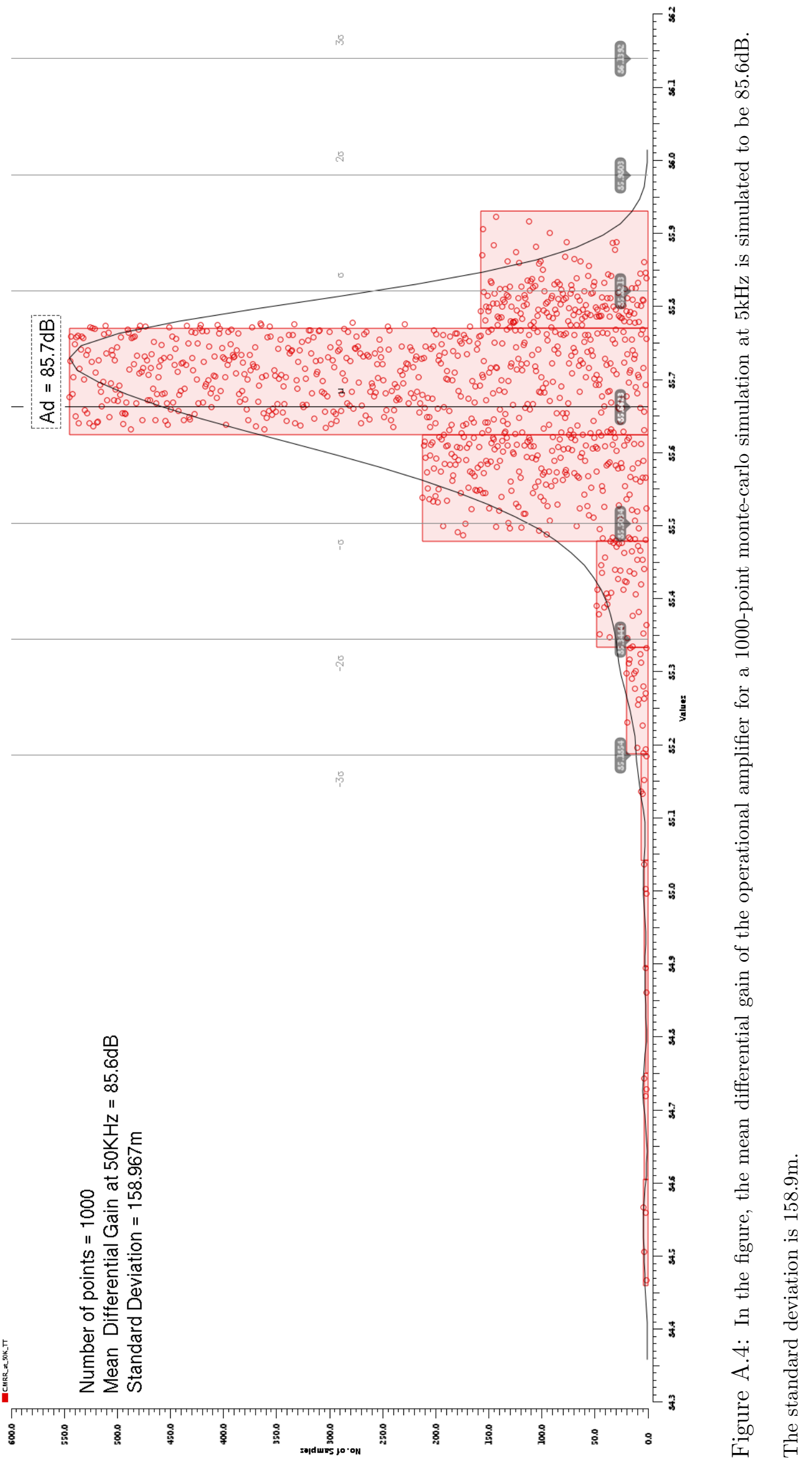




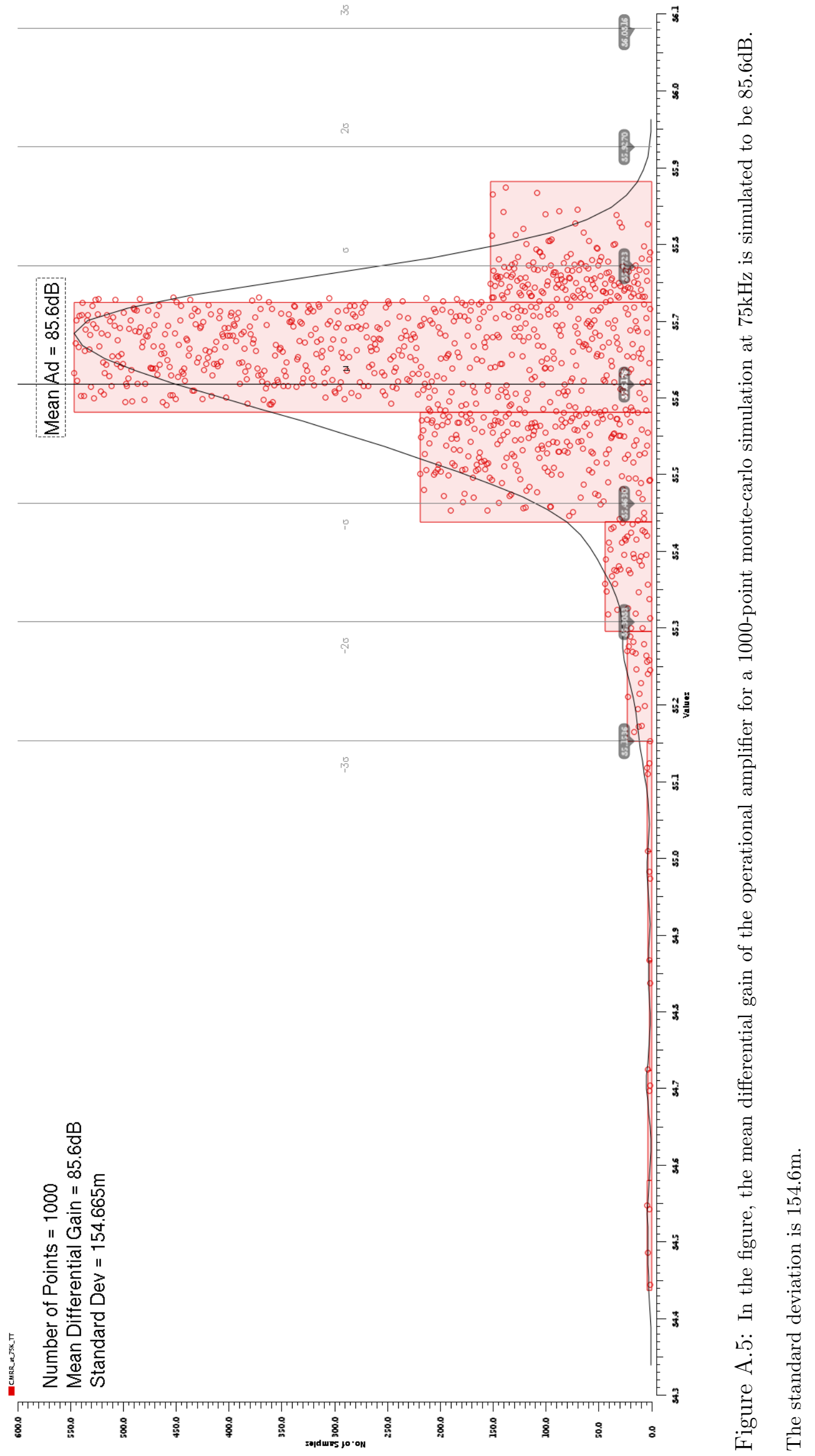




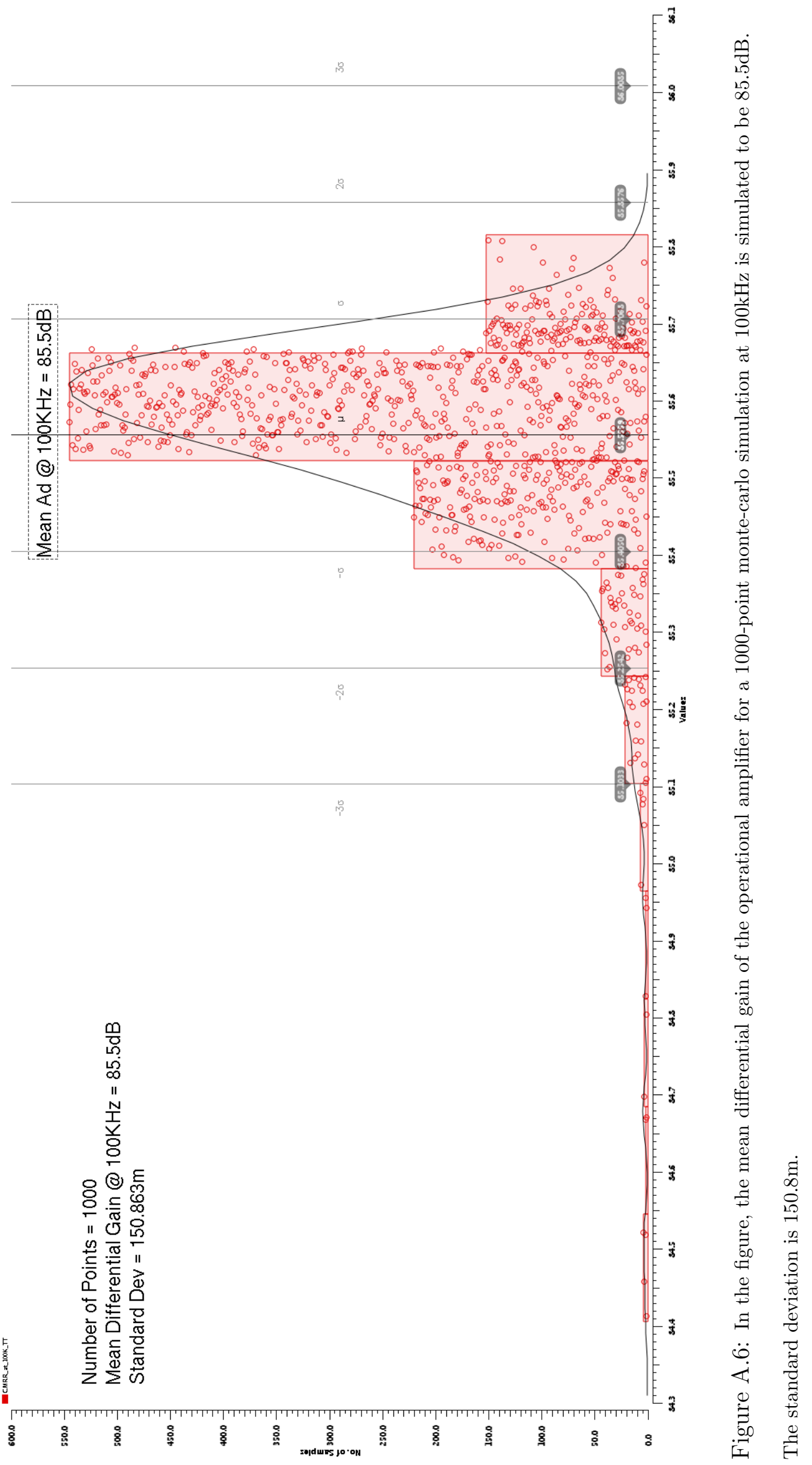




\section{A.2 Common Mode Rejection}

A common mode input of $1 \mathrm{mV}$ amplitude is applied at the non-inverting and inverting terminals of the operational amplifier. For a single supply voltage of $1.5 \mathrm{~V}$ and $0 \mathrm{~V}$ as the ground reference, monte-carlo simulations for differential gain of the operational amplifier is found at frequencies $1 \mathrm{kHz}, 5 \mathrm{kHz}, 10 \mathrm{kHz}, 50 \mathrm{kHz}$, $75 \mathrm{kHz}$ and $100 \mathrm{kHz}$.

The following figures illustrate the common mode gain at respective frequencies for a 1000-point monte-carlo simulation. 


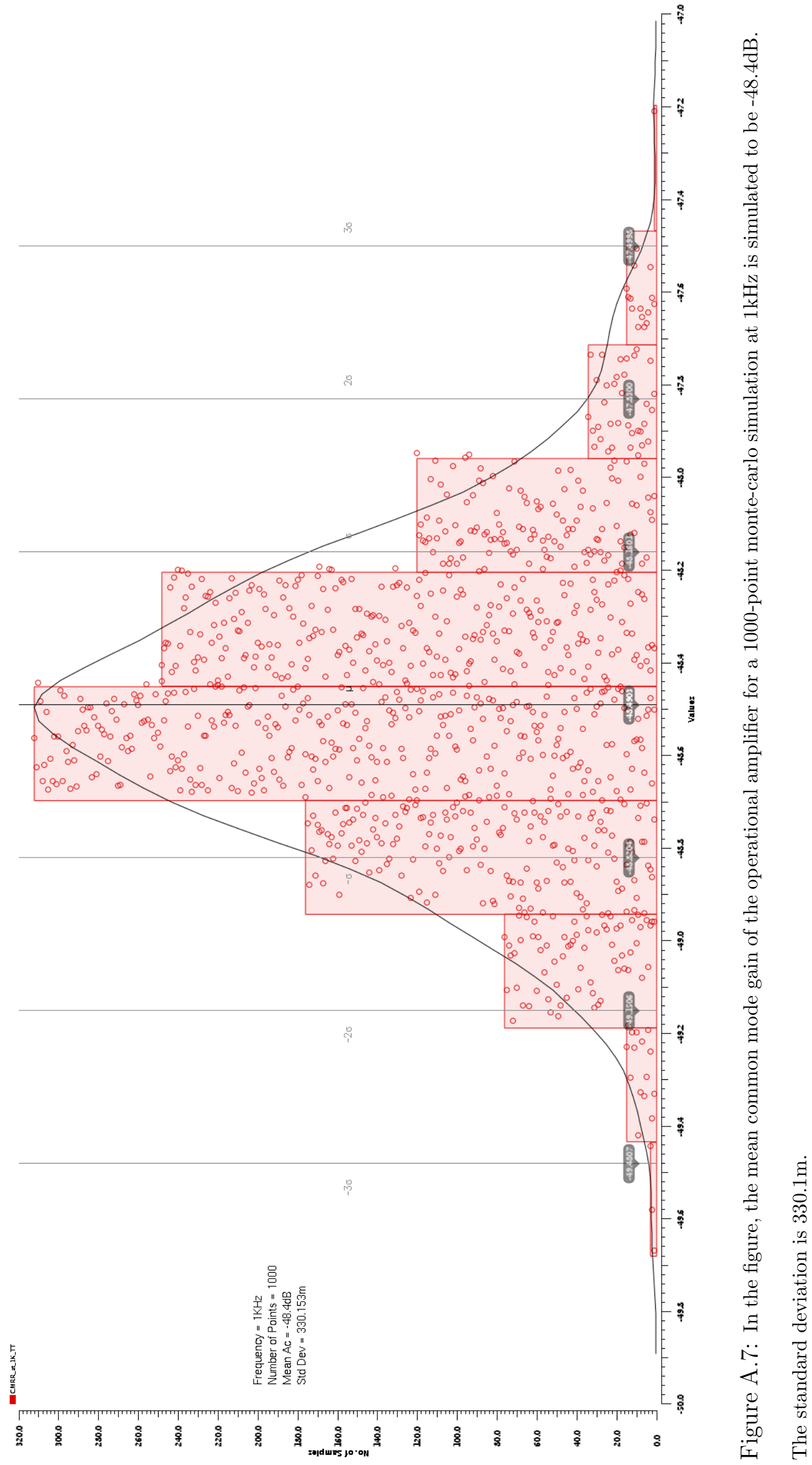




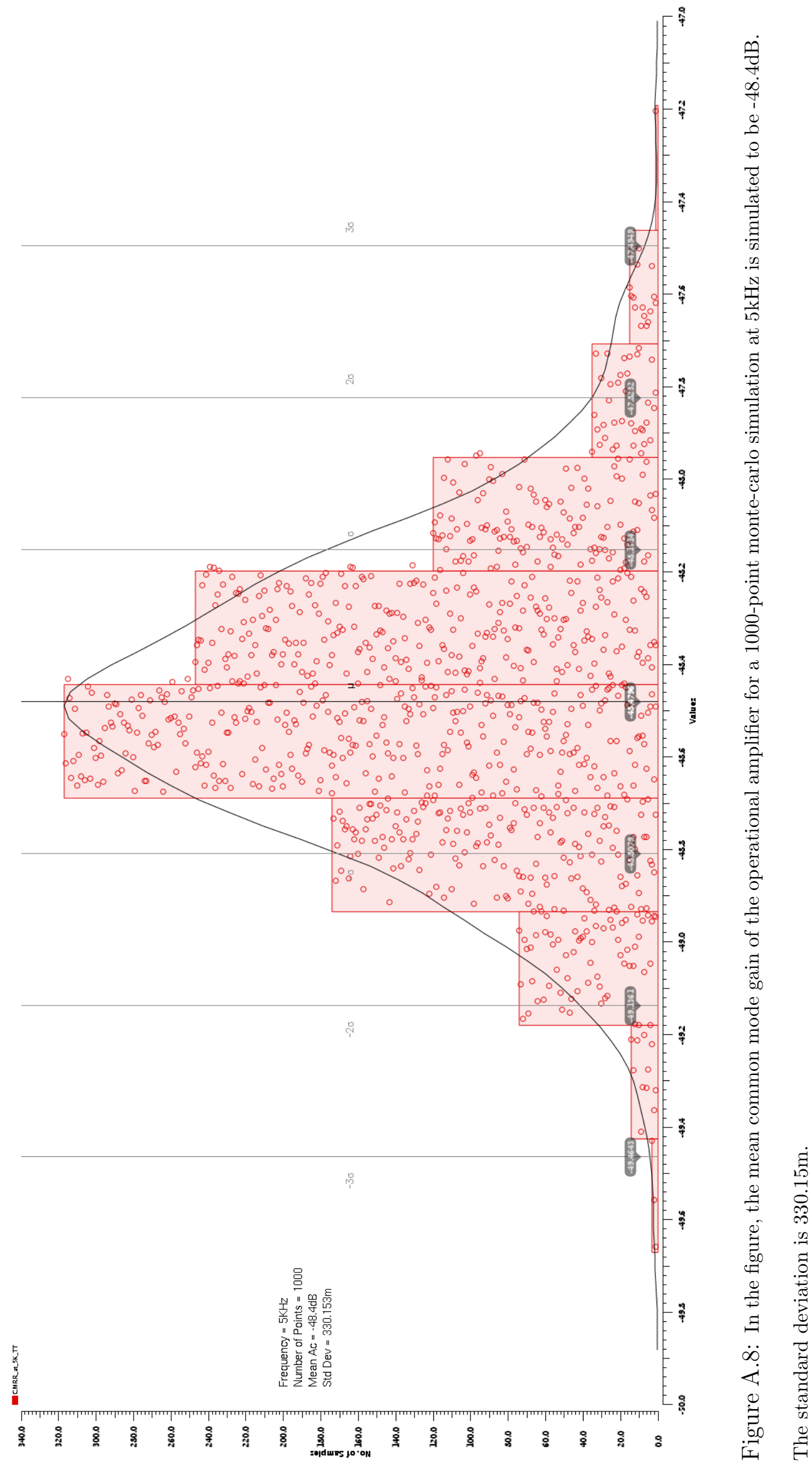




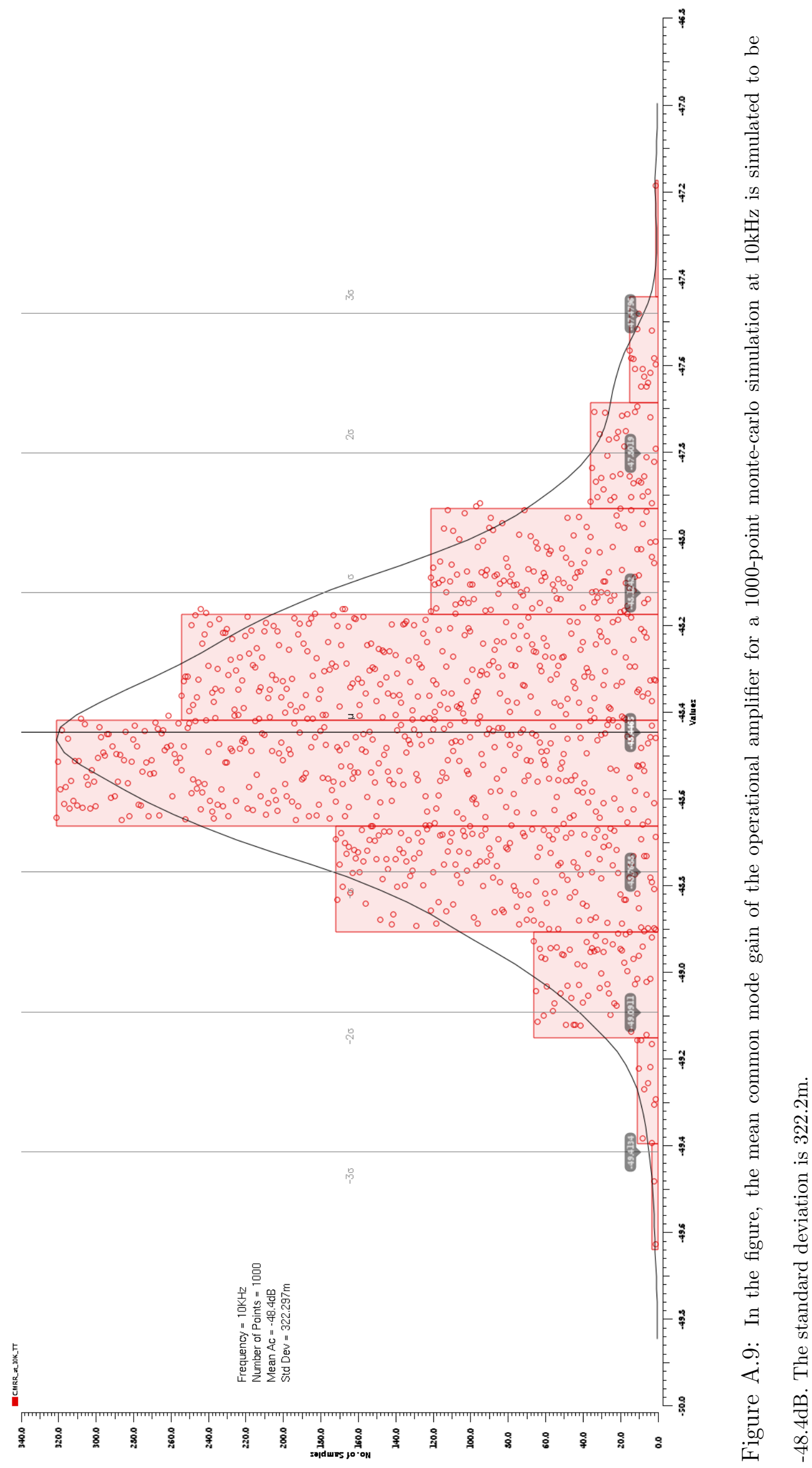




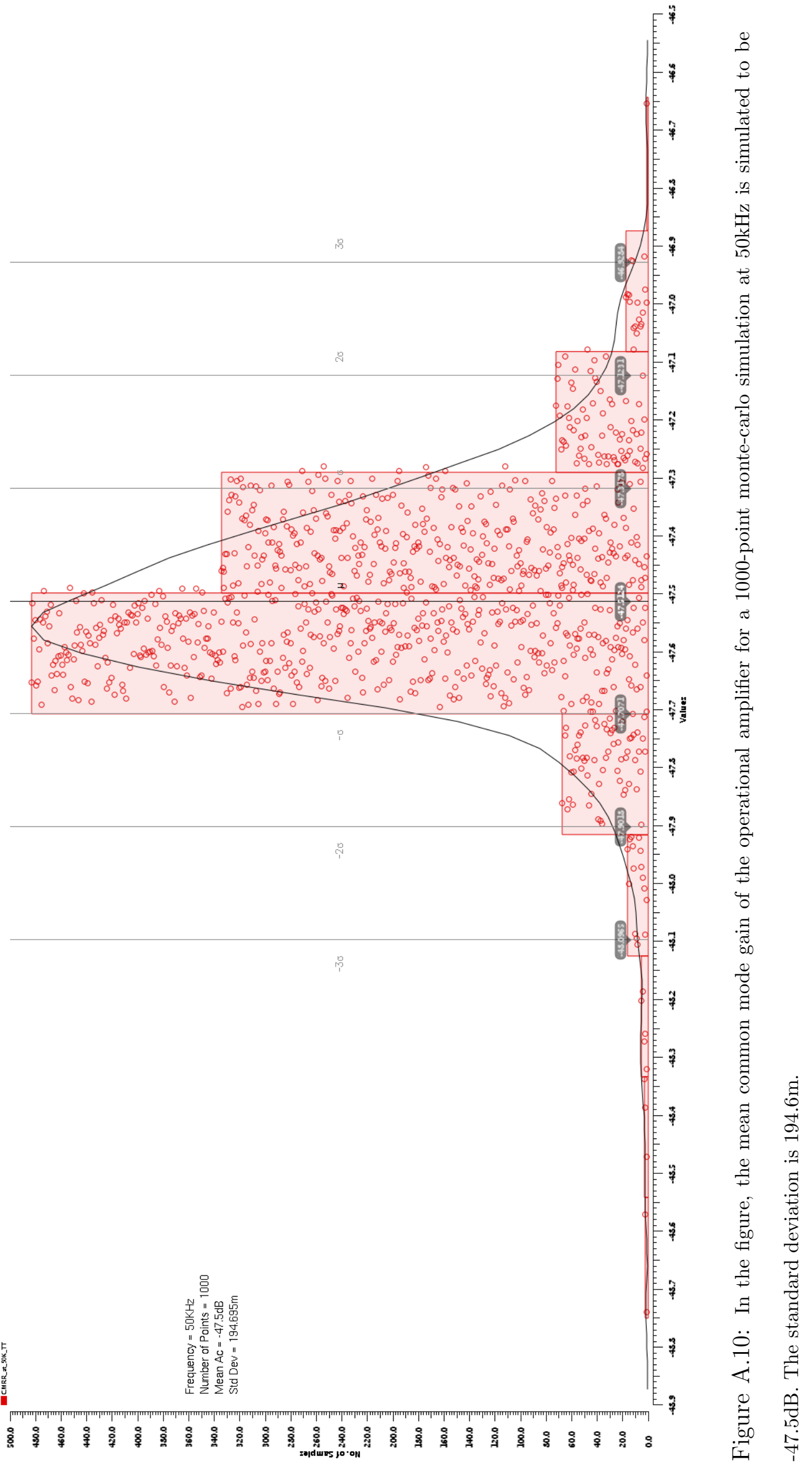




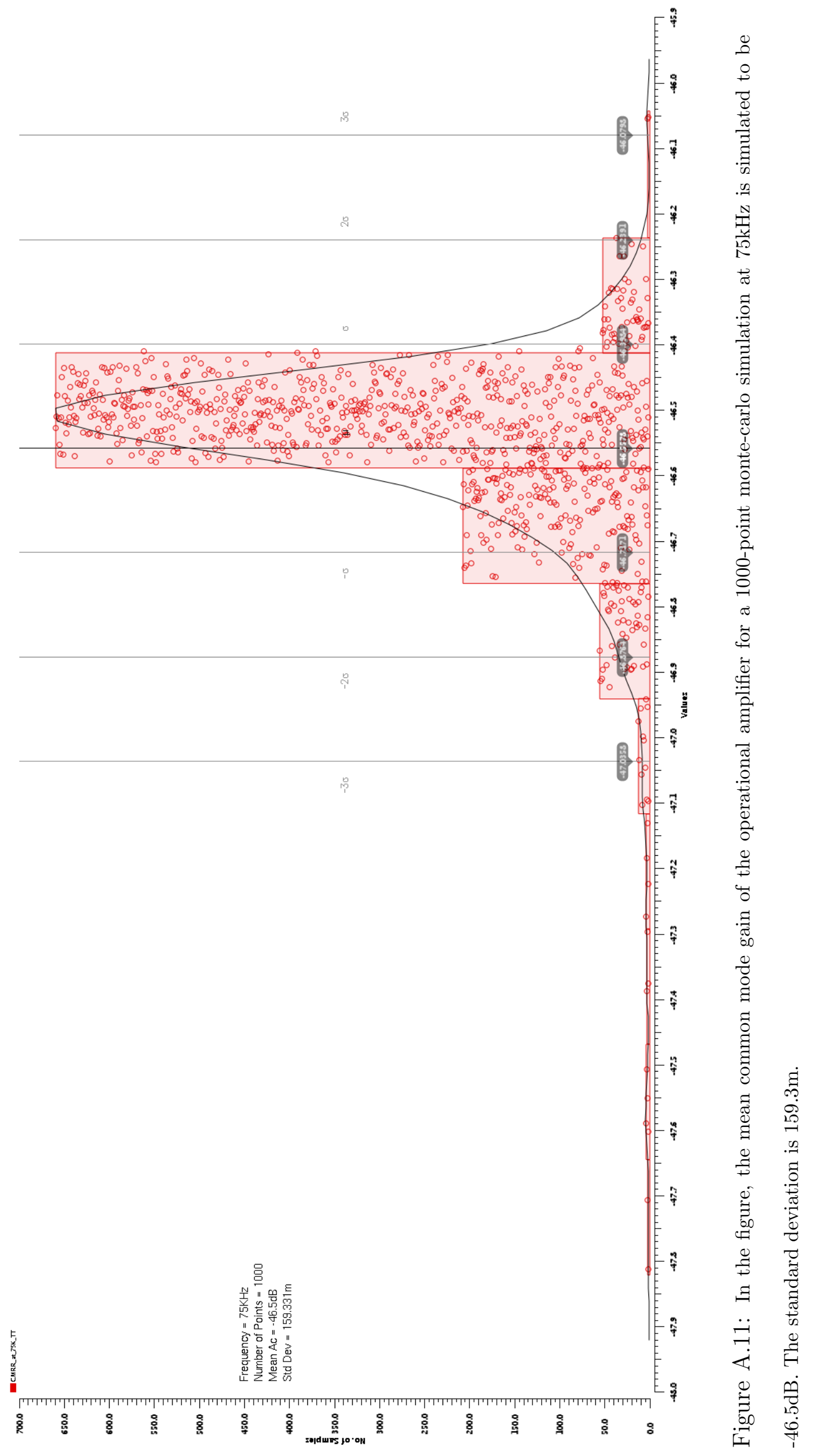




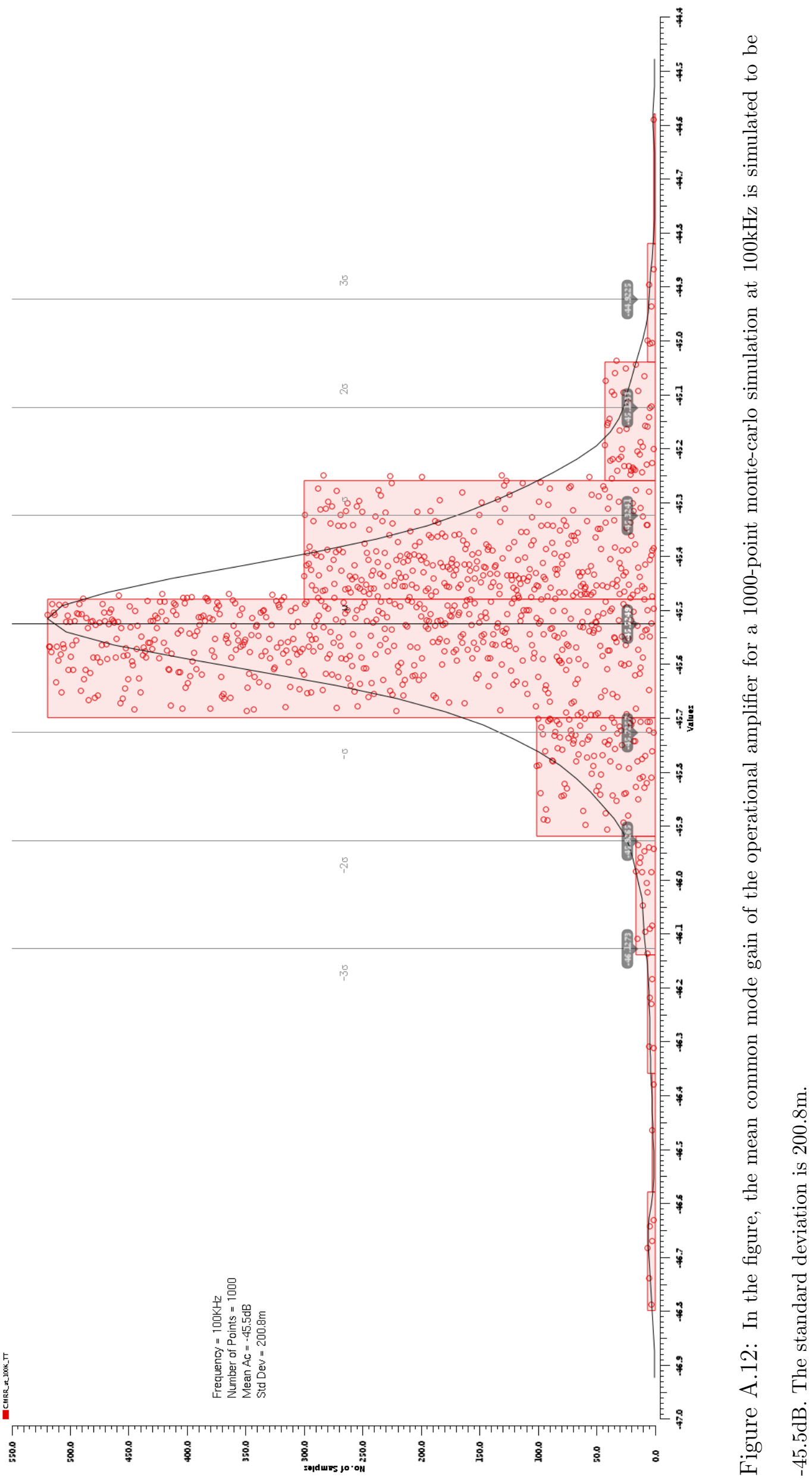


From the figures A.1 to A.12, the mean differential gain, $A_{D}$, and the common mode gain, $A_{C}$, of the operational amplifier as well as the common mode rejection ratio(CMRR) at different frequencies is outlined in the table A.1.

\begin{tabular}{|c|c|c|c|}
\hline Frequency $(\mathrm{kHz})$ & Differential gain $(\mathrm{dB})$ & Common mode gain $(\mathrm{dB})$ & CMRR $(\mathrm{dB})$ \\
\hline 1 & 85.7 & -48.4 & 134.1 \\
\hline 5 & 85.6 & -48.4 & 134 \\
\hline 10 & 85.7 & -48.4 & 134.1 \\
\hline 50 & 85.6 & -47.5 & 133.1 \\
\hline 75 & 85.6 & -46.5 & 132.1 \\
\hline 100 & 85.5 & -45.5 & 131 \\
\hline
\end{tabular}

Table A.1: Differential gain, common mode gain and common mode rejection ratio of the operational amplifier at different frequencies 
At corners TT, FF and SS, the differential gain and common mode gain of the operational amplifier are simulated at $10 \mathrm{kHz}$ and outlined in the table A.2. The common mode rejection ratio is calculated and shown in the table.

\begin{tabular}{|c|c|c|c|}
\hline Corner & Differential gain $(\mathrm{dB})$ & Common mode gain $(\mathrm{dB})$ & CMRR $(\mathrm{dB})$ \\
\hline $\mathrm{TT}$ & 85.7 & -48.4 & 134.1 \\
\hline $\mathrm{FF}$ & 85.9 & -50.2 & 136.1 \\
\hline $\mathrm{SS}$ & 84.4 & -46.9 & 131.3 \\
\hline
\end{tabular}

Table A.2: Differential gain, common mode gain and common mode rejection ratio of the operational amplifier for a $1 \mathrm{mV}$ input signal of $10 \mathrm{kHz}$ frequency at TT, FF and SS corners 


\section{Appendix B}

\section{Bioimpedance Sytem}

\section{Multi-frequency Simulations}

The transient behavior of the bioimpedance system for an input of $10 \mathrm{kHz}$ frequency is seen in the Chapter 3. From the transient behavior the unknown impedance is calculated to be $1 \mathrm{~K} \Omega$. In this appendix, the transient behavior of the bioimpedance system at frequencies $5 \mathrm{kHz}, 50 \mathrm{kHz}$ and $100 \mathrm{kHz}$ is shown and the unknown impedance is calculated.

In all the cases, the input applied to the mirrored modified Howland current source is $100 \mathrm{mV}$ peak-to-peak. This causes the current source to inject a peak current of $50 \mu \mathrm{A}$ for a $5 \mathrm{kHz}$ input signal.

The current injected for a $50 \mathrm{kHz}$ and the $100 \mathrm{kHz}$ is $250 \mu \mathrm{A}$. The magnitude of the current chosen to keep the output voltages within the voltage ranges of the circuit. The following figure illustrate the input, intermediate and output signals of the bioimpedance system. 


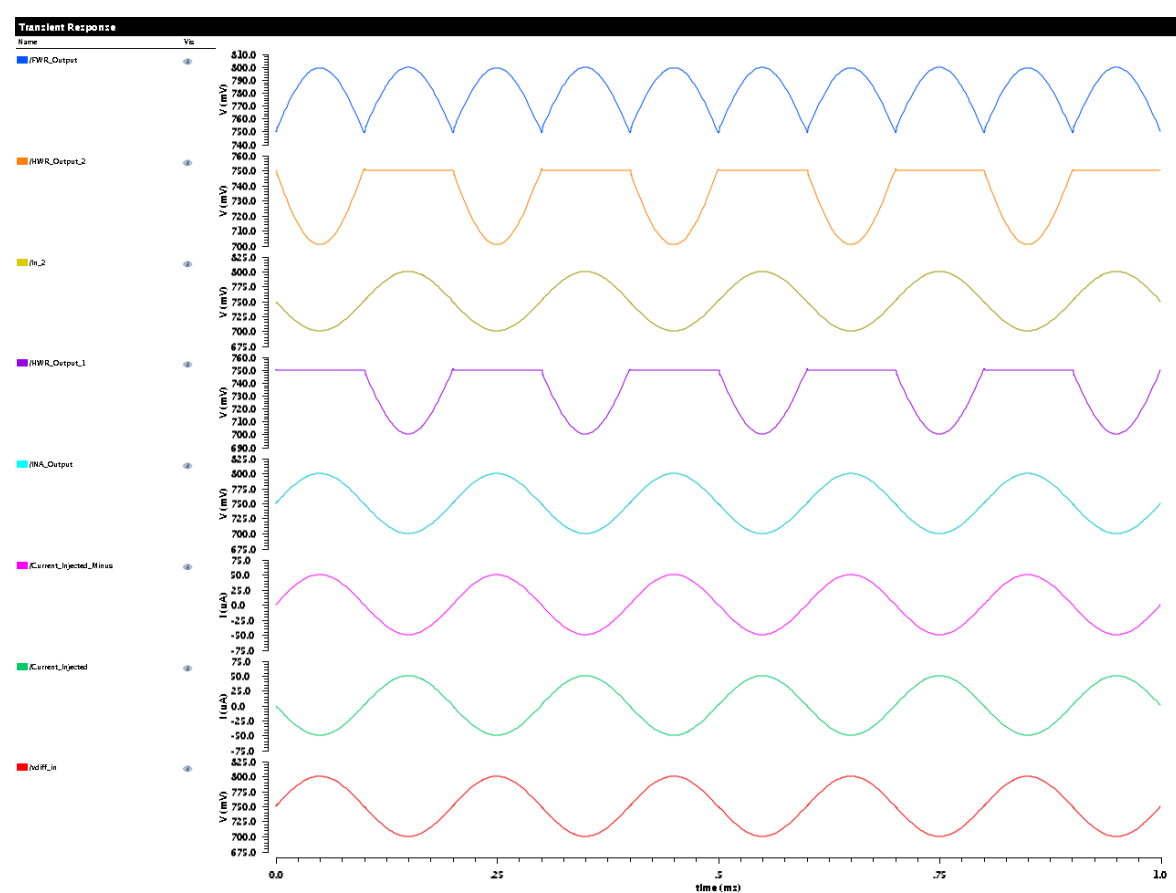

Figure B.1: From top to bottom: Fully rectified output signal of amplitude 50mV, phaseshifter half-wave rectifier output of amplitude $50 \mathrm{mV}$, output of the unity gain inverting amplifier, half-wave rectifier output of amplitude $50 \mathrm{mV}$, instrumentation amplifier output with amplitude of $50 \mathrm{mV}, 50 \mu \mathrm{A}$ peak current injected (leaving the unknown impedance), $50 \mu \mathrm{A}$ peak current injected (entering the unknown impedance), $50 \mathrm{mV}$ peak input signal of $5 \mathrm{kHz}$ frequency

Therefore, unknown impedance is

$$
Z_{\text {Unknown }}=\frac{V_{\text {rectified_RMS }}}{I_{R M S}}=\frac{35.3 \mathrm{mV}}{35.3 \mu \mathrm{A}}=1 \mathrm{~K} \Omega
$$




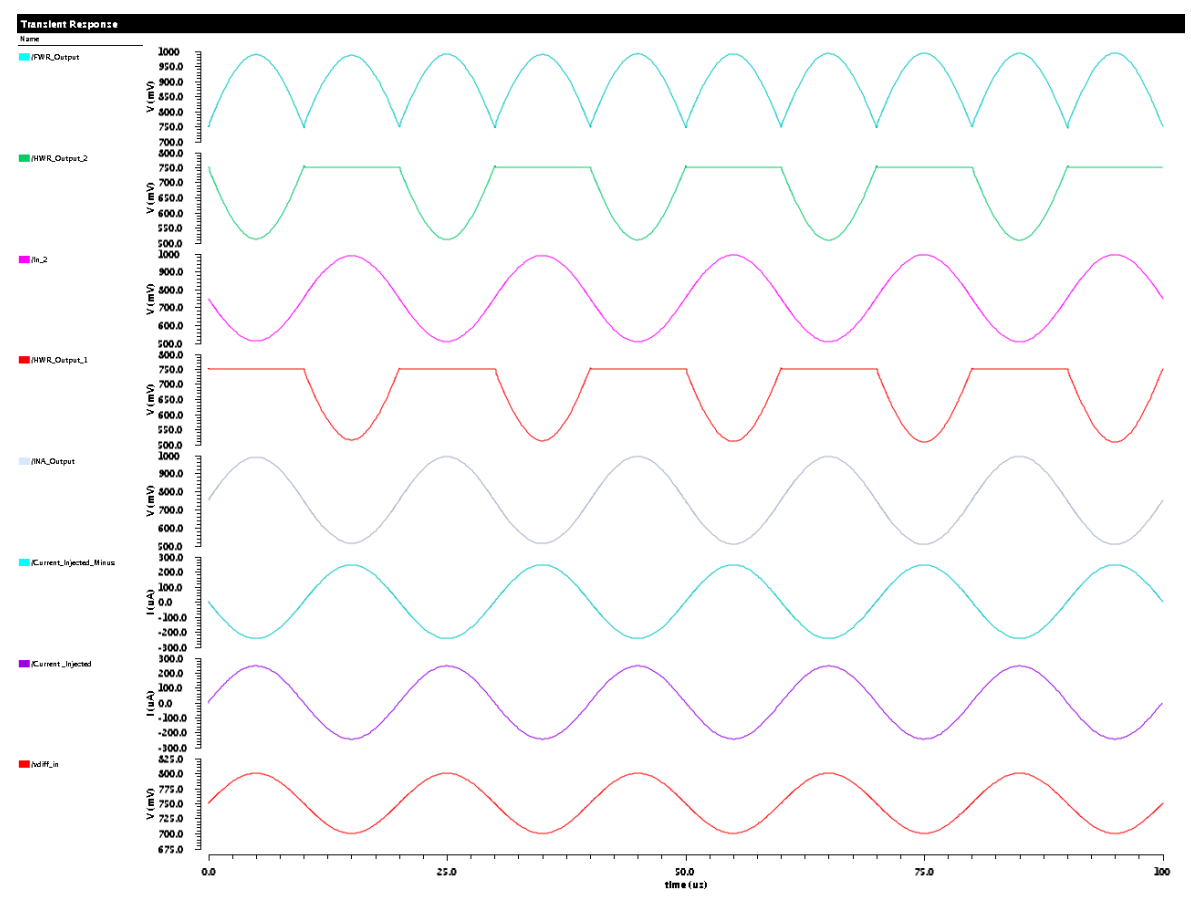

Figure B.2: From top to bottom: Fully rectified output signal of amplitude $250 \mathrm{mV}$, phaseshifter half-wave rectifier output of amplitude $250 \mathrm{mV}$, output of the unity gain inverting amplifier, half-wave rectifier output of amplitude $250 \mathrm{mV}$, instrumentation amplifier output with amplitude of $250 \mathrm{mV}, 250 \mu \mathrm{A}$ peak current injected (leaving the unknown impedance), $250 \mu \mathrm{A}$ peak current injected (entering the unknown impedance), $50 \mathrm{mV}$ peak input signal of $50 \mathrm{kHz}$

Therefore, unknown impedance is

$$
Z_{\text {Unknown }}=\frac{V_{\text {rectified_RMS }}}{I_{R M S}}=\frac{176.7 \mathrm{mV}}{176.6 \mu \mathrm{A}}=1 \mathrm{~K} \Omega
$$




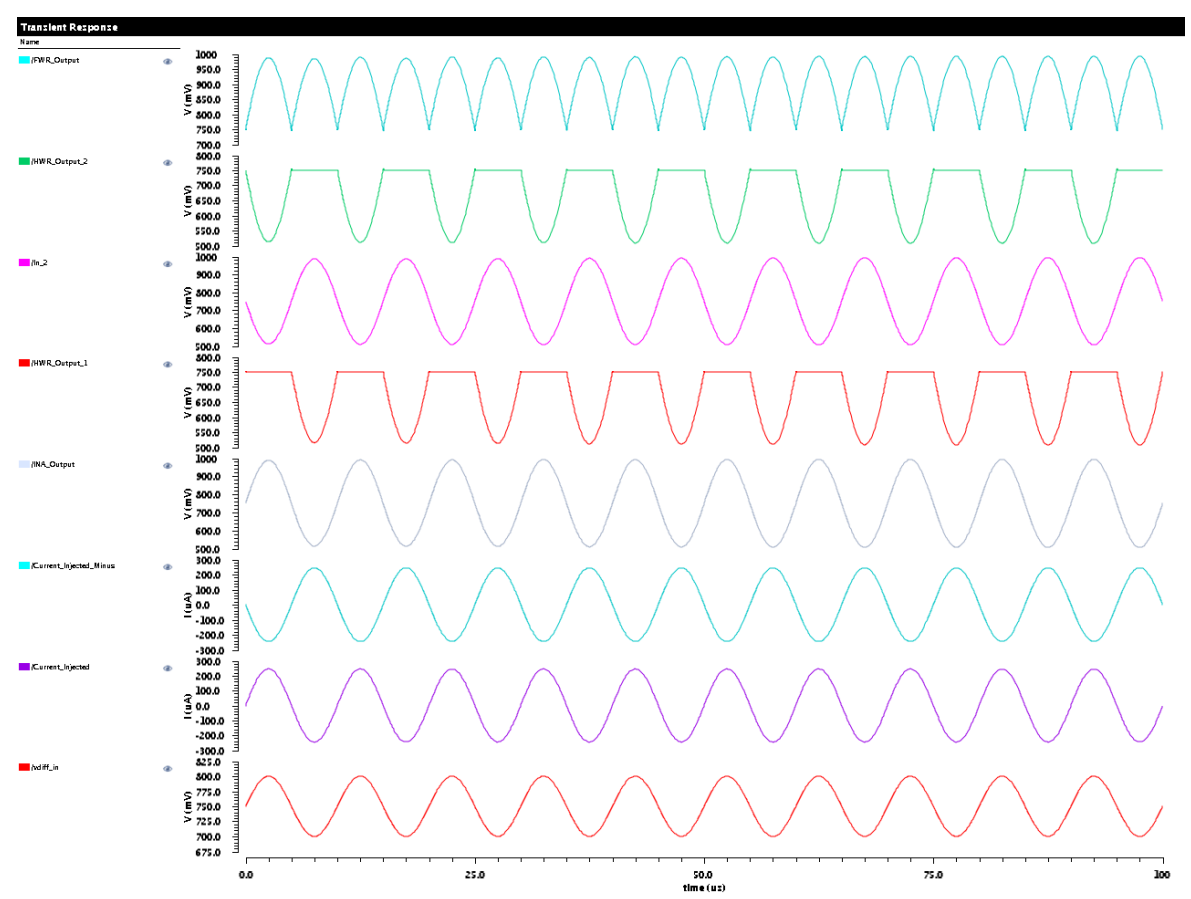

Figure B.3: From top to bottom: Fully rectified output signal of amplitude $250 \mathrm{mV}$, phaseshifter half-wave rectifier output of amplitude $250 \mathrm{mV}$, output of the unity gain inverting amplifier, half-wave rectifier output of amplitude $250 \mathrm{mV}$, instrumentation amplifier output with amplitude of $250 \mathrm{mV}, 250 \mu \mathrm{A}$ peak current injected (leaving the unknown impedance), $250 \mu \mathrm{A}$ peak current injected (entering the unknown impedance), $50 \mathrm{mV}$ peak input signal of $100 \mathrm{kHz}$

Therefore, unknown impedance is

$$
Z_{\text {Unknown }}=\frac{V_{\text {rectified_RMS }}}{I_{R M S}}=\frac{176.7 m \mathrm{~V}}{176.6 \mu \mathrm{A}}=1 \mathrm{~K} \Omega
$$

The unknown impedance in all the cases is calculated from the RMS value of the fully rectified output and RMS value of the injected current. The unknow impedance is found to be $1 \mathrm{~K} \Omega$. 


\section{Bibliography}

[1] K. R. Foster and H. C. Lukaski, "Whole-body impedance-what does it measure?," The American journal of clinical nutrition, vol. 64, no. 3, pp. 388S396S, 1996.

[2] C. Margo, J. Katrib, M. Nadi, and A. Rouane, "A four-electrode low frequency impedance spectroscopy measurement system using the ad5933 measurement chip," Physiological measurement, vol. 34, no. 4, p. 391, 2013.

[3] R. F. Kushner, R. Gudivaka, and D. A. Schoeller, "Clinical characteristics influencing bioelectrical impedance analysis measurements.," The American journal of clinical nutrition, vol. 64, no. 3, pp. 423S-427S, 1996.

[4] R. Plonsey and R. C. Barr, Bioelectricity: a quantitative approach. Springer Science \& Business Media, 2007.

[5] T. Instruments, "A comprehensive study of the howland current pump," $A N$ $1515 A, 2008$.

[6] P. Bertemes-Filho, V. C. Vincence, M. S. Santos, and I. X. Zanatta, "Low power current sources for bioimpedance measurements: A comparison between howland and integrated cmos ota circuits," Journal of Electrical Bioimpedance, vol. 3, no. 1, pp. 66-73, 2012. 
[7] T. Instruments, "Datasheet-afe4300-low-cost, integrated analog front-end for weight-scale and body composition measurement," Dallas, Texas, USA, 2012.

[8] STMicroelectronics, "Datasheet hm301d-diagnostic-quality acquisition system for bio-electric sensors and bio-impedance measurements," 2015.

[9] M. H. Hamzah, A. B. Jambek, and U. Hashim, "Design and analysis of a two-stage cmos op-amp using silterra's $0.13 \mu \mathrm{m}$ technology," in Computer Applications and Industrial Electronics (ISCAIE), 2014 IEEE Symposium on, pp. 55-59, IEEE, 2014.

[10] A. Pajkanovic, "A 130 nm operational amplifier: Design and schematic level simulation," in Computational Intelligence, Communication Systems and Networks (CICSyN), 2015 7th International Conference on, pp. 249-254, IEEE, 2015.

[11] J. M. Carrillo, G. Torelli, M. A. Domínguez, R. Pérez-Aloe, J. M. Valverde, and J. F. Duque-Carrillo, "A family of low-voltage bulk-driven cmos continuous-time cmfb circuits," IEEE Transactions on Circuits and Systems II: Express Briefs, vol. 57, no. 11, pp. 863-867, 2010.

[12] H. F. Achigui, C. J. B. Fayomi, and M. Sawan, "1-v dtmos-based classab operational amplifier: implementation and experimental results," IEEE Journal of Solid-State Circuits, vol. 41, no. 11, pp. 2440-2448, 2006.

[13] H. Yazdanian, M. M. Samani, and A. Mahanm, "Characteristics of the howland current source for bioelectric impedance measurements systems," in Biomedical Engineering (ICBME), 2013 20th Iranian Conference on, pp. 189193, IEEE, 2013. 
[14] A. Yúfera and A. Rueda, "A method for bioimpedance measure with four-and two-electrode sensor systems," in Engineering in Medicine and Biology Society, 2008. EMBS 2008. 30th Annual International Conference of the IEEE, pp. 2318-2321, IEEE, 2008.

[15] P. Batra, S. Singh, and S. P. Singh, "A new method for measurement of bioelectrical impedance," in Communication Systems and Network Technologies (CSNT), 2011 International Conference on, pp. 740-743, IEEE, 2011.

[16] F. Seoane, J. Ferreira, J. J. Sanchéz, and R. Bragós, "An analog front-end enables electrical impedance spectroscopy system on-chip for biomedical applications," Physiological measurement, vol. 29, no. 6, p. S267, 2008.

[17] U. G. Kyle, I. Bosaeus, A. D. De Lorenzo, P. Deurenberg, M. Elia, J. M. Gómez, B. L. Heitmann, L. Kent-Smith, J.-C. Melchior, M. Pirlich, et al., "Bioelectrical impedance analysisópart i: review of principles and methods," Clinical nutrition, vol. 23, no. 5, pp. 1226-1243, 2004.

[18] K. R. Aroom, M. T. Harting, C. S. Cox, R. S. Radharkrishnan, C. Smith, and B. S. Gill, "Bioimpedance analysis: a guide to simple design and implementation," Journal of Surgical Research, vol. 153, no. 1, pp. 23-30, 2009.

[19] B. J. Nordbotten, "Bioimpedance measurements using the integrated circuit ad5933," 2008.

[20] D. Holder, "Appendix a. brief introduction to bioimpedance," Electrical Impedance Tomography: Methods, History and Applications, vol. 411, 2004. 
[21] C. A. Prior, C. R. Rodrigues, A. L. Aita, J. B. dos Santos Martins, and F. C. B. Vieira, "Design of an integrated low power high cmrr instrumentation amplifier for biomedical applications," Analog Integrated Circuits and Signal Processing, vol. 57, no. 1-2, pp. 11-17, 2008.

[22] H.-C. Chow and J.-Y. Wang, "High cmrr instrumentation amplifier for biomedical applications," in Signal Processing and Its Applications, $200 \%$. ISSPA 200\%. 9th International Symposium on, pp. 1-4, IEEE, 2007.

[23] U. Pliquett and A. Barthel, "Interfacing the ad5933 for bio-impedance measurements with front ends providing galvanostatic or potentiostatic excitation," in Journal of Physics: Conference Series, vol. 407, p. 012019, IOP Publishing, 2012.

[24] "Obesity and overweight," sept 2014.

[25] M. A. Khaled, M. Khatun, M. Haque, I. Kabir, and D. Mahalanabis, "Single, dual and multi-frequency bioimpedance to measure human body composition," in Engineering in Medicine and Biology Society, 1995 and 14th Conference of the Biomedical Engineering Society of India. An International Meeting, Proceedings of the First Regional Conference., IEEE, pp. 1/87-1/88, Feb 1995.

[26] M. Khaled, M. Khatun, M. Haque, I. Kabir, and D. Mahalanabis, "Single, dual and multi-frequency bioimpedance to measure human body composition," in Engineering in Medicine and Biology Society, 1995 and 14th Conference of the Biomedical Engineering Society of India. An International Meet- 
ing, Proceedings of the First Regional Conference., IEEE, pp. 1-87, IEEE, 1995.

[27] C. Gabriel, S. Gabriel, and E. Corthout, "The dielectric properties of biological tissues: 1. literature survey phys," Med. Biol. 1996a, vol. 41, pp. 22312249 .

[28] S. Jain, "To design high cmrr, high slew rate instrumentation amplifier using ota and cdta for biomedical application," International Journal of Engineering Research, vol. 2, no. 5, pp. 332-336, 2013.

[29] A. Devices, "Ad5933 1 msps, 12-bit impedance converter," Network Analyzer, 2005.

[30] A. Ivorra, "Bioimpedance monitoring for physicians: an overview," Centrale Nationale de Microélectronique, 2003.

[31] B. Greenebaum and F. S. Barnes, Bioengineering and Biophysical Aspects of Electromagnetic Fields. CRC press, 2006.

[32] S. Grimnes and O. Martinsen, Bioelectricity and bioimpedance basics. Academic, 2008.

[33] J. R. Claycomb and J. Q. P. Tran, Introductory biophysics: Perspectives on the living state. Jones \& Bartlett Learning, 2010.

[34] M. R. Neuman, "Biopotential amplifiers," Medical instrumentation: application and design, pp. 316-318, 1998.

[35] M. A. G. Lorenzo, A. A. S. Manzano, M. T. A. Gusad, J. R. E. Hizon, and M. D. Rosales, "Design and implementation of cmos rail-to-rail opera- 
tional amplifiers," in Communications and Information Technologies, $200 \%$. ISCIT'07. International Symposium on, pp. 61-66, IEEE, 2007.

[36] R. J. Baker, CMOS: circuit design, layout, and simulation, vol. 1. John Wiley \& Sons, 2008.

[37] P. Bertemes-Filho, L. Negri, A. Felipe, and V. Vincence, "Mirrored modified howland circuit for bioimpedance applications: Analytical analysis," in Journal of Physics: Conference Series, vol. 407, p. 012030, IOP Publishing, 2012.

[38] B. Carter, "Understanding op amp parameters," App. Note from Texas Inst, 2001.

[39] Ø. G. Martinsen, O. Pabst, C. Tronstad, and S. Grimnes, "Sources of error in ac measurement of skin conductance," Journal of Electrical Bioimpedance, vol. 6, no. 1, pp. 49-53, 2015.

[40] S. Gabriel, R. Lau, and C. Gabriel, "The dielectric properties of biological tissues: Ii. measurements in the frequency range 10 hz to 20 ghz," Physics in medicine and biology, vol. 41, no. 11, p. 2251, 1996.

[41] M. Osypka and E. Gersing, "Tissue impedance spectra and the appropriate frequencies for eit," Physiological measurement, vol. 16, no. 3A, p. A49, 1995. 